(c) 2014

Brian George Tice

ALL RIGHTS RESERVED 


\title{
MEASUREMENT OF NUCLEAR DEPENDENCE IN INCLUSIVE CHARGED CURRENT NEUTRINO SCATTERING
}

\author{
By \\ BRIAN GEORGE TICE \\ A Dissertation submitted to the \\ Graduate School-New Brunswick \\ Rutgers, The State University of New Jersey \\ in partial fulfillment of the requirements \\ for the degree of \\ Doctor of Philosophy \\ Graduate Program in Physics and Astronomy \\ written under the direction of \\ Professor Ronald D. Ransome \\ and approved by
}

New Brunswick, New Jersey

January, 2014 


\title{
ABSTRACT OF THE DISSERTATION
}

\section{Measurement of Nuclear Dependence in Inclusive Charged Current Neutrino Scattering by BRIAN GEORGE TICE}

\author{
Dissertation Director: \\ Professor Ronald D. Ransome
}

Neutrino experiments use heavy nuclei $(\mathrm{C}, \mathrm{Fe}, \mathrm{Pb})$ to achieve necessary statistics. However, the use of heavy nuclei exposes these experiments to the nuclear dependence of neutrino-nucleus cross sections, which are poorly known and difficult to model.

This dissertation presents an analysis of the nuclear dependence of inclusive chargedcurrent neutrino scattering using events in carbon, iron, lead, and scintillator targets of the MINERvA detector. MINERvA (Main INjector ExpeRiment for $\nu$-A) is a few-GeV neutrinonucleus scattering experiment at Fermilab. Acceptance of the muon spectrometer limits the analysis to $2 \mathrm{GeV}<E_{\nu}<20 \mathrm{GeV}$ and $0^{\circ}<\theta_{\mu}<17^{\circ}$. The total cross section $\sigma$ and differential cross section $\frac{d \sigma}{d x_{b j}}$ are measured, but the final result is ratios of these cross sections. Taking ratios dramatically reduces systematic uncertainties, most notably from the flux. This is the first precision measurement of nuclear effects using neutrino interactions in the nonperturbative, few-GeV regime.

The modeling of nuclear-dependence is found to be adequate as a function of neutrino energy, but fails to describe the $x_{b j}$-dependence. The data show an enhancement in the cross section at $x_{b j}>0.7$ which grows with the size of the nucleus and a suppression of the cross section at $x_{b j}<0.1$ which also grows with the size of the nucleus. Neither the enhancement nor the suppression are predicted by current neutrino interaction simulation codes. 


\section{Acknowledgements}

Above all, I thank my advisor Professor Ronald Ransome for providing me with the guidance and sparking the drive I needed to succeed in my graduate studies. His calm focus and soft touch are responsible for my growth from a student of the utmost ignorance to a physicist eager to reveal the mysteries of the universe. I struggle to imagine being matched to better mentor.

It takes a village to produce a dissertation on neutrino cross sections. I am indebted to every member of the MINERvA collaboration. I owe an especially tremendous debt to Elaine Schulte, Dave Schmitz, Gabe Perdue, and Mousumi Datta whose knowledge, wisdom, and patience I greedily exploited. They demonstrated the highest level of scientific acumen and gave every assistance in my effort to rise to their proficiency. Kevin McFarland, Jorge Morfín, and Debbie Harris, the MINERvA spokespersons, deserve special praise for their commitment to ensuring the success of our experiment, and I'm grateful that each of them somehow still made time to provide a steady stream of good advice during my time at Fermilab. It was my great fortune to have skilled and helpful colleagues Heidi Schellman, Erica Snider, and Laura Fields, who taught me the ropes of scientific computing. All divisions of Fermilab have my sincere appreciation, especially those in Computing Division to whom I sent many a service request.

Words cannot express the gratitude I have to my family for their constant love and support. I know you are proud of me and never grow tired of working to deserve your praise. Lastly, I marvel at my beautiful and brilliant wife Deidre for being all I need, all the time. The push through late nights and early mornings was made easy by just thinking of you. 


\section{Table of Contents}

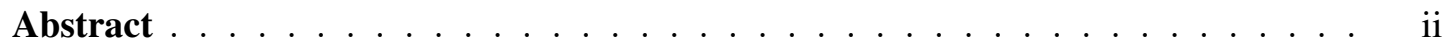

Acknowledgements ............................. ii

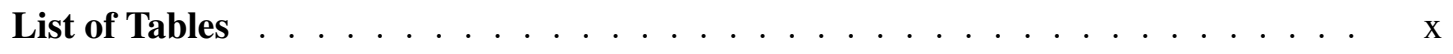

List of Figures . . . . . . . . . . . . . . . . . . . . . . . . . . . . . . . . . . xii

1. Introduction and Overview $\ldots \ldots \ldots \ldots \ldots \ldots$

1.1. The Standard Model . . . . . . . . . . . . . . . . . . . . . . 2

1.1.1. Shortcomings of the Standard Model . . . . . . . . . . . . . 3

1.2. Neutrino Scattering . . . . . . . . . . . . . . . . . . . 6

1.2.1. Quasi-Elastic Scattering . . . . . . . . . . . . . . . 7

1.2.2. Resonance Production . . . . . . . . . . . . . . . . . 8

1.2.3. Deep Inelastic Scattering . . . . . . . . . . . . . . . . . . . 9

1.3. Nuclear Effects . . . . . . . . . . . . . . . . . . . . . . . . . . . 10

1.3.1. Primary Neutrino Interaction . . . . . . . . . . . . . . . 11

1.3.2. Final State Interactions . . . . . . . . . . . . . . . . . . . . . . . 14

2. Apparatus . . . . . . . . . . . . . . . . . . . . . . . 16

2.1. NuMI Neutrino Beam . . . . . . . . . . . . . . . . . . . . . . . . 16

2.1.1. Proton Delivery . . . . . . . . . . . . . . . . . . . . . 17

2.1.2. NuMI Target and Horns . . . . . . . . . . . . . . . . . 18

2.1.3. Decay and Detector Halls $\ldots \ldots \ldots$ 
2.2. The MINERvA Detector $\ldots \ldots \ldots \ldots \ldots \ldots \ldots \ldots \ldots$

2.2.1. Upstream Region . . . . . . . . . . . . . . . . . . . . 22

2.2.2. Inner Detector $\ldots \ldots \ldots \ldots \ldots \ldots \ldots$

2.2.3. Outer Detector . . . . . . . . . . . . . . . . . . 27

2.2.4. Scintillator and Light Collection . . . . . . . . . . . . . . 28

2.2.5. Electronics and Data Acquisition. . . . . . . . . . . . . . . . 29

2.3. MINOS Near Detector $\ldots \ldots \ldots \ldots \ldots \ldots$

2.4. Data Run Periods . . . . . . . . . . . . . . . . . . 31

3. Calibration and Reconstruction of MINERvA $\ldots \ldots \ldots \ldots$

3.1. Hit Calibration . . . . . . . . . . . . . . . . . . . 34

3.1.1. Ex situ Calibrations . . . . . . . . . . . . . . . . . . . . 36

3.1.2. In situ Calibrations . . . . . . . . . . . . . . . . . . . . . . 38

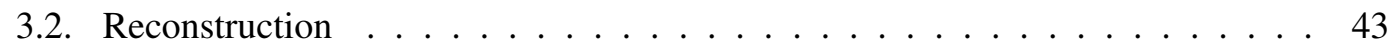

3.2.1. Clustering Hits by Time $\ldots \ldots \ldots \ldots \ldots \ldots$

3.2.2. Clustering Hits by Position . . . . . . . . . . . . . . . . 44

3.2.3. Track-Based Event Formation . . . . . . . . . . . . . . . . 45

3.2.4. Track Formation . . . . . . . . . . . . . . . . . . . 47

3.2.5. Track and Vertex Fitting $\ldots \ldots \ldots \ldots \ldots \ldots$

3.2.6. Muon Reconstruction. . . . . . . . . . . . . . . . . . . . . . . . . 49

3.2.7. Recoil System Reconstruction _ . . . . . . . . . . . . . . . 50

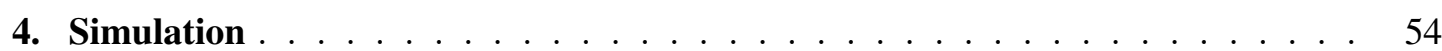

$4.1 . \quad$ NuMI Flux $\ldots \ldots \ldots \ldots \ldots \ldots \ldots \ldots$

$4.1 .1 . \quad$ Resulting Flux $\ldots \ldots \ldots \ldots \ldots$. . . . . . . . . . . . 57

4.2. Neutrino Interactions . . . . . . . . . . . . . . . . . . . . . . . 57 
4.2.1. Nuclear Physics . . . . . . . . . . . . . . . . . . . . . 57

4.2.2. Cross Section Models $\ldots \ldots \ldots \ldots$. . . . . . . . . . 58

4.2.3. Final State Interactions . . . . . . . . . . . . . . . 63

4.3. MINERvA Simulation . . . . . . . . . . . . . . . . . . . . . 64

4.3.1. Propagation of Particles in MINERvA . . . . . . . . . . . . . . 64

4.3.2. Overlaying Simulated Events onto Data Gates . . . . . . . . . . . . . 64

4.3.3. MINERvA Readout and Electronics . . . . . . . . . . . . . . . 65

4.4. MINOS Simulation . . . . . . . . . . . . . . . . . . 65

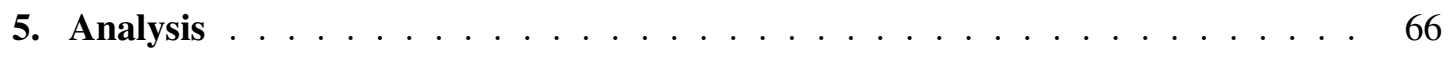

5.1. Selection of Charged Current $\nu_{\mu}$ Events . . . . . . . . . . . . 66

5.1.1. Muon Requirements . . . . . . . . . . . . . . . . . . . . 67

5.1.2. Fiducial Volume and Target Identification . . . . . . . . . . . . . 68

5.1.3. Reconstruction Quality Assurance . . . . . . . . . . . . . . . . . 72

5.2. Event Sample . . . . . . . . . . . . . . . . . . . . 73

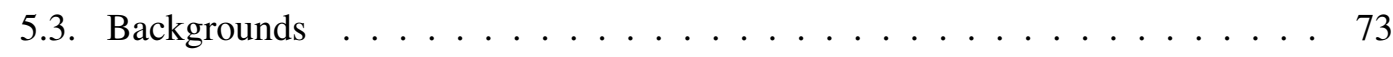

5.3.1. Wrong Sign and Neutral Current Events . . . . . . . . . . . . . . . 74

5.3.2. Rock Muon Events . . . . . . . . . . . . . . . . . . . 75

5.3.3. Misidentified Nucleus . . . . . . . . . . . . . . 75

5.4. Scintillator Contamination Subtraction . . . . . . . . . . . . . . . . 77

5.4.1. Geometric Acceptance Correction . . . . . . . . . . . . . . . 77

5.4.2. Hadronic Energy Efficiency Correction . . . . . . . . . . . . . 80

5.4 .3$. Accuracy . . . . . . . . . . . . . . . . 82

5.5. Calculating a Cross Section . . . . . . . . . . . . . . . . . . 84

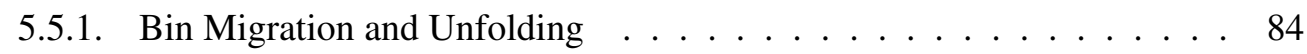

5.5.2. Efficiency and Acceptance Corrections . . . . . . . . . . . . 85 
5.5.3. Flux . . . . . . . . . . . . . . . . . . . . 89

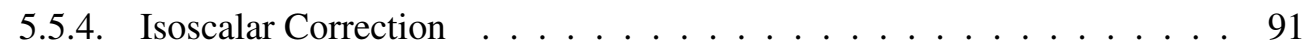

5.5.5. Absolute Cross Section Results … . . . . . . . . . . . 92

5.5.6. Forming Ratios $\ldots \ldots \ldots \ldots \ldots \ldots \ldots$

5.5.7. Summing Over Targets . . . . . . . . . . . . . . . . . . . 94

5.6. Systematic Uncertainties … . . . . . . . . . . . . . . . . 95

5.6.1. Evaluation of Systematic Errors _ . . . . . . . . . . . . . 95

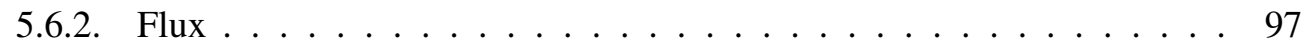

5.6.3. Muon Energy . . . . . . . . . . . . . . . . . . . . . . 100

5.6.4. Muon Vertex and Angle Reconstruction . . . . . . . . . . . . . . 103

5.6.5. Recoil Energy Reconstruction . . . . . . . . . . . . . . . . 103

5.6.6. Subtraction of Scintillator Contamination . . . . . . . . . . . . 113

5.6.7. Unfolding . . . . . . . . . . . . . . . . . . . . . 114

5.6.8. Normalization . . . . . . . . . . . . . . . . . . . 114

5.6.9. Efficiency Correction . . . . . . . . . . . . . . . . . 115

5.6.10. Cancellation of Errors in Ratios $\ldots \ldots \ldots \ldots \ldots$

6. Results and Conclusions $\ldots \ldots \ldots \ldots \ldots$

6.1. Results. . . . . . . . . . . . . . . . . . . . . . . . . . . . . 119

6.1.1. Neutrino Energy Result. . . . . . . . . . . . . . . . . . . . . . 119

6.1.2. Bjorken x Result . . . . . . . . . . . . . . . . . . . . 125

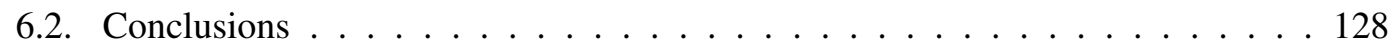

Appendix A. Additional Figures $\ldots \ldots \ldots \ldots$

A.1. Kinematic Distributions of Selected Events ． . . . . . . . . . . . . 130

A.2. Predicted Neutral Current and Wrong Sign Background . . . . . . . . . . . 148 
A.3. Event Sample Vertex Distributions . . . . . . . . . . . . . . . . . . . . 154

A.4. Scintillator Background Prediction . . . . . . . . . . . . . . . . . 172

A.4.1. Scale Factors . . . . . . . . . . . . . . . . . . . . 172

A.4.2. Accuracy of Scintillator Background Prediction . . . . . . . . . . . . 172

A.4.3. Errors on Scintillator Background Prediction . . . . . . . . . . . 172

A.5. Signal and Background Prediction Distributions . . . . . . . . . . . . . 172

A.6. Efficiency . . . . . . . . . . . . . . . . . . . . . 195

A.7. Migration . . . . . . . . . . . . . . . . . . . . . . . 213

A.8. Absolute Cross Sections ．. . . . . . . . . . . . . . . . . . . . . . . . 219

A.9. Cross Section Ratios for All Targets . . . . . . . . . . . . . . . . . . . . 224

A.9.1. Neutrino Energy . . . . . . . . . . . . . . . . . . . . 224

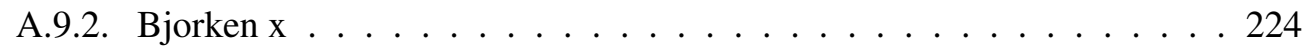

Appendix B. Nuclear Target Geometry Calculations … . . . . . . . . . . . 228

B.1. Fraction Along Strip Adjacent to Passive Target Occupied by C/Fe/Pb . . . . . 228

B.1.1. Inner Detector Border ． . . . . . . . . . . . . . . . 228

B.1.2. Nuclear Target Materials Border . . . . . . . . . . . . . . . . . . 229

B.2. Fiducial Area of Nuclear Targets . . . . . . . . . . . . . . . . . 232

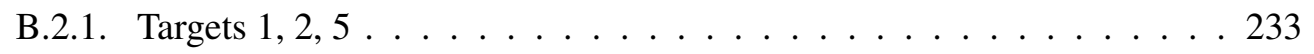

B.2.2. Target 3 . . . . . . . . . . . . . . . . 234

Appendix C. Kalman Filters $\ldots \ldots \ldots \ldots$

C.1. Track Fitting . . . . . . . . . . . . . . . 236

C.1.1. Seed State . . . . . . . . . . . . . . . . . . . . . . . 237

C.1.2. Predict . . . . . . . . . . . . . . . . . . 237

C.1.3. Filter . . . . . . . . . . . . . . . . . . . . 239 


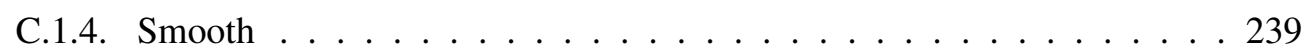

C.2. Vertex Fitting . . . . . . . . . . . . . . . . . . . . 239

C.2.1. Initial Guess . . . . . . . . . . . . . . . . . . 239

C.2.2. Adaptive Vertex Kalman Filter . . . . . . . . . . . . . . . . . . . 240

C.2.3. Annealing . . . . . . . . . . . . . . . . . . . . . . 242

Appendix D. The MINERvA Collaboration . . . . . . . . . . . . . . . . . . . 243

Bibliography . . . . . . . . . . . . . . . . . . 247 


\section{List of Tables}

1.1. Forces described by the Standard Model and their mediator bosons. . . . . . . 3

1.2. Properties of quarks and leptons. . . . . . . . . . . . . . . 3

2.1. Relevant meson decays in NuMT. . . . . . . . . . . . . . . . . . . . . 19

2.2. Modular composition of subdetectors in the inner detector. . . . . . . . . . . 23

2.3. Density properties of a scintillator plane. . . . . . . . . . . . . . . . 24

2.4. Nuclear composition of a scintillator plane. . . . . . . . . . . . . . . . 24

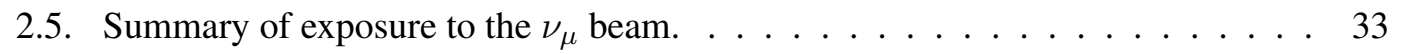

3.1. $d E / d x$ for materials in the MINERvA detector. . . . . . . . . . . . . . 52

3.2. Calorimetric scale factors $\alpha \ldots \ldots \ldots \ldots \ldots \ldots$

4.1. Summary of hadron production interactions constrained by measurements. . . . 56

4.2. A summary of relativistic Fermi gas parameters in GENIE. . . . . . . . . . . 58

5.1. Muon tracking efficiency in MINOS. . . . . . . . . . . . . . . . . . 68

5.2. Mass and number of scatterers in the fiducial volume of each target material. . . 71

5.3. Neutrino events selected in the carbon, iron, lead, and scintillator chargedcurrent sample. . . . . . . . . . . . . . . . . . . . . 73

5.4. Muon tracking efficiency corrections and errors. . . . . . . . . . . . . . . 88

5.5. Neutrino flux prediction. . . . . . . . . . . . . . . . . 90

5.6. Modules used for scintillator measurements in ratio of cross sections. . . . . . 94

5.7. Uncertainties on NuMI beamline components. . . . . . . . . . . . . . . . . 100

5.8. Uncertainties on muon momentum reconstruction. . . . . . . . . . . . . . . 101 
5.9. Uncertainties on final state interaction models in GENIE. . . . . . . . . . . . . 105

5.10. Uncertainties on the detector response to different sources of light. . . . . . . . 109

5.11. Uncertainties on neutrino cross section model in GENIE. . . . . . . . . . . . 116

6.1. Comparison of measured to expected values for $\sigma^{A} / \sigma^{C H}$ as functions of $E_{\nu}$. . 123

6.2. Comparison of measured to expected values for $\frac{d \sigma^{A}}{d x_{b j}} / \frac{d \sigma^{C H}}{d x_{b j}}$ as functions of $x_{b j}$. 124

6.3. Grouping of systematic errors for display purposes. . . . . . . . . . . . . . . 124 


\section{List of Figures}

2.1. A schematic drawing of the NuMI beamline. . . . . . . . . . . . . . . . 17

2.2. The NuMI target. . . . . . . . . . . . . . . . . . . 18

2.3. Positions of the NuMI baffle, target, and horns. . . . . . . . . . . . . . . 19

2.4. The NuMI secondary absorbers and monitors. . . . . . . . . . . . . . . . . 20

2.5. Major regions of the MINERvA detector. . . . . . . . . . . . . . 21

2.6. An inner detector tracking module mounted in an outer detector frame. . . . . . 22

2.7. Cross sectional view of triangular scintillator strips in a plane. . . . . . . . . . 24

2.8. Schematic of the nuclear targets of MINERvA. . . . . . . . . . . . . 25

2.9. Cross-sectional view of a pair of outer detector scintillator scripts. . . . . . . . 28

2.10. The MINOS near detector. . . . . . . . . . . . . . . 31

2.11. NuMI beam data recorded by MINERvA. . . . . . . . . . . . . . 32

3.1. Schematic of effects accounted for in hit calibration . . . . . . . . . . . 35

3.2. Schematic of the Rutgers PMT test stand. . . . . . . . . . . . . . . . . 37

3.3. Alignment of a scintillator plane. . . . . . . . . . . . . . . . . . 40

3.4. Consistency in energy response after channel-to-channel calibration. . . . . . . 41

3.5. Calibration of absolute energy scale using muon energy unit. . . . . . . . . . . 42

3.6. Hit time distribution and reconstructed time slices. . . . . . . . . . . . . . 44

4.1. A comparison of NA49 and FTFP_BERT predicted hadron cross sections. . . . 56

4.2. Prediction of the flux with hadron reweighting. . . . . . . . . . . . . . 57

4.3. A comparison of total cross section data to GENIE. . . . . . . . . . . . . . . 59 
4.4. Bodek-Yang modifications to nuclear structure functions. . . . . . . . . . . . 61

5.1. Carbon event candidate with one reconstructed track. . . . . . . . . . . 70

5.2. Lead event candidate with two reconstructed tracks and 3D vertex. . . . . . . . 71

5.3. Backgrounds from neutral current and wrong sign events. . . . . . . . . . . 75

5.4. Predicted background from misidentified nuclei. . . . . . . . . . . . . . . 76

5.5. Distribution showing misidentification of the interaction nucleus. . . . . . . . . 78

5.6. Ratio of vertex-z distribution in data to simulation. . . . . . . . . . . . . 78

5.7. Muon acceptance as a function of muon energy and angle. . . . . . . . . . . 80

5.8. Muon acceptance as a function of transverse position. . . . . . . . . . . . . . 81

5.9. $\quad E_{h a d}$-dependent scale factor in prediction of background from scintillator. . . . 82

5.10. Accuracy of the prediction of scintillator contamination. . . . . . . . . 83

5.11. Bin migration. . . . . . . . . . . . . . . . . 85

5.12. Event selection efficiency and purity. . . . . . . . . . . . . . . . . . . . . 89

5.13. Neutrino flux prediction. . . . . . . . . . . . . . . . . . 91

5.14. Isoscalar correction applied each cross sections. . . . . . . . . . . . . . . . . 92

5.15. Isoscalar correction applied each ratio of cross sections. . . . . . . . . . . . 93

5.16. Measured absolute scintillator cross section. . . . . . . . . . . . . . . . . 93

5.17. Comparison of cross section ratios measurements from each target. . . . . . . . 95

5.18. Flux errors on charged current inclusive event rate. . . . . . . . . . . . . 98

5.19. Flux errors from spread in hadronic interaction models. . . . . . . . . . . . . . 99

5.20. Error on flux due to NuMI beamline systematic uncertainties. . . . . . . . . . . 101

5.21. Residual in the MINOS reconstruction of inverse muon momentum. . . . . . . 102

5.22. Residual in the position of the muon vertex. . . . . . . . . . . . . . . . . . 104

5.23. Residual in the slopes of the muon track. . . . . . . . . . . . . . . . . . . . 104

5.24. Test beam study of Birks' parameter with stopped protons. . . . . . . . . . . . 108 
5.25. Sources of visible energy in hits of the recoil system. . . . . . . . . . . . . 109

5.26. Test beam measurement of proton response. . . . . . . . . . . . . . . . . 110

5.27. Discrepancy between data and simulation in the $n A \rightarrow p X$ cross section. . . . 110

5.28. Michel electron energy spectrum. . . . . . . . . . . . . . . . . . . 112

5.29. Test beam measurement of charged pion response. . . . . . . . . . . . . . . 112

5.30. Scintillator background scale error. . . . . . . . . . . . . . . . . . . . . 114

5.31. Cross section model errors on reconstruction efficiency. . . . . . . . . . . . . . 117

5.32. Summary of relative systematic errors on the reconstruction efficiency. . . . . . 117

5.33. Error cancellation in cross section ratio measurement. . . . . . . . . . . . . . 118

6.1. Measurement of ratios of $\nu_{\mu}$ charged current inclusive $\sigma . \ldots \ldots \ldots$

6.2. Measurement of ratios of $\nu_{\mu}$ charged current inclusive $\frac{d \sigma}{d x_{b j}}$. . . . . . . . . 121

6.3. Relative errors on ratios of $\sigma$ and $\frac{d \sigma}{d x_{b j}}$. . . . . . . . . . . . 122

A.1. Kinematic distributions of selected events. . . . . . . . . . . . . . . . . . 131

A.2. Predicted neutral current and wrong sign backgrounds. . . . . . . . . . . . . 148

A.3. Vertex distributions of selected events. . . . . . . . . . . . . . . . 155

A.4. $E_{h a d}$-dependent scale factor in prediction of background from scintillator in passive targets. . . . . . . . . . . . . . . . . . . 173

A.5. Accuracy of the prediction of scintillator contamination for $E_{\nu}$ distributions. . . 174

A.6. Accuracy of the prediction of scintillator contamination for $x_{b j}$ distributions. $\quad 175$

A.7. Systematic errors on the scintillator background prediction. . . . . . . . . . . 176

A.9. Signal and background distribution plots. . . . . . . . . . . . . . 178

A.10.Efficiency and errors on efficiency. . . . . . . . . . . . . . . . . 196

A.11.Bin migration. . . . . . . . . . . . . . . . . . . . . . . 213

A.12. Absolute cross sections. . . . . . . . . . . . . . . . . . . . 220

A.13. Comparison of cross section ratios in each $E_{\nu}$ bin. . . . . . . . . . . . . 225 
A.15.Comparison of cross section ratios in each $x_{b j}$ bin. . . . . . . . . . . . 227

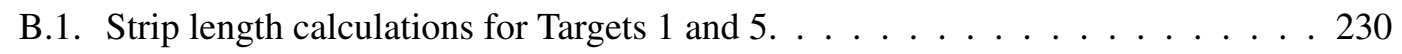

B.2. Strip length calculations for Target 2. . . . . . . . . . . . . . . . 231

B.3. Strip length calculations for Target 3. . . . . . . . . . . . . . . . . . 232 


\section{Chapter 1}

\section{Introduction and Overview}

Particle physics is the study of fundamental properties of matter and interactions. It seeks to provide the ultimate answer to the questions "what's that made of?" and "how was it made?" The scattering of one particle by another has been one of the most successful tools in discovering answers to these questions and understanding the most basic building blocks of the universe. Scattering has provided proof for the existence of increasingly fundamental constituents of matter: atoms $\rightarrow$ nuclei $\rightarrow$ nucleons $\rightarrow$ quarks. The Standard Model of particle physics is the most complete explanation of the subatomic universe, but it does not describe all phenomena.

Neutrino scattering has played, and continues to play, a particularly important role in the development of the Standard Model. The first observation of the neutral current weak interactions of neutrinos in 1973 was crucial in verifying the electroweak theory that unified the electromagnetic and weak forces. Measurements of neutrinos scattering off the constituents of the nucleon were crucial in the development of the Quark Parton Model. More recently, neutrino oscillations have been used to study fundamental symmetries of the universe. Unique properties of the neutrino make it a good candidate for understanding the decades-old mystery of the EMC effect, which remains unexplained, despite significant study using charged lepton probes.

This chapter describes aspects of the Standard Model, neutrino scattering, and the nuclear environment that are relevant to charged current scattering of neutrinos at few-GeV energies ${ }^{1}$

\footnotetext{
1 "Few-GeV" in neutrino scattering is a loosely defined energy regime of roughly $1-5 \mathrm{GeV}$, where quasi-elastic scattering, resonance production, and deep inelastic scattering (c.f. Sec. 1.2) are all relevant. MINERvA is referred to as a "few-GeV" experiment, even though the actual range of measured energies is larger.
} 
Shortcomings of current models are presented to motivate this measurement, which improves the situation. The remaining chapters in this dissertation are as follows:

- Chapter 2 describes the neutrino beam and detectors used to collect data for this measurement.

- Chapter 3 summarizes calibration and event reconstruction in the MINERvA detector.

- Chapter 4 details the methods used to simulate neutrino interactions in MINERvA.

- Chapter 5 explains the method used to analyze the nuclear dependence of charged current inclusive $\nu_{\mu}$ interactions with a description of systematic uncertainties.

- Chapter 6 presents and discusses the results of the analysis.

\subsection{The Standard Model}

The Standard Model of particle physics explains three of the four observable forces: the electromagnetic, strong, and weak forces. It defines $16+\left.1\right|^{2}$ fundamental particles. There are three types of particle: bosons, quarks, and leptons.

Bosons have integer spin. Four of these are spin-1 vector bosons which mediate the forces of the Standard Model: the photon $(\gamma)$, gluon $(g), W$, and $Z$. The forces are summarized in Table 1.1. The fifth boson is the scalar Higgs boson $(H)$ which has spin 0 and is responsible for the property of mass.

Quarks and leptons are fermions, which have half-integer spin. There are 6 quarks and 6 leptons, which come in pairs that are repeated for three generations. The quark generations are up $(u)$ and down $(d)$, charm $(c)$ and strange $(s)$, top $(t)$ and bottom $(b)$. There two types of leptons: charged leptons and neutrinos. The lepton generations are electron $(e)$ and electron neutrino $\left(\nu_{e}\right)$, muon $(\mu)$ and muon neutrino $\left(\nu_{\mu}\right)$, tau $(\tau)$ and tau neutrino $\left(\nu_{\tau}\right)$. For each of

\footnotetext{
${ }^{2}$ Quarks, leptons, and force carriers make 16. The Higgs is the one.
} 


\begin{tabular}{ccc}
\hline Force & Particle & Example \\
\hline Strong & Gluon $(g)$ & Binds the quarks in a nucleon \\
Electromagnetic & photon $(\gamma)$ & Holds atoms together in molecules \\
Weak & $\mathrm{W}$ and $\mathrm{Z}\left(W^{ \pm}\right.$and $\left.Z^{0}\right)$ & Beta decay \\
\hline
\end{tabular}

Table 1.1: Forces described by the Standard Model and their mediator bosons.

\begin{tabular}{ccccccc}
\hline & \multicolumn{7}{c}{ Leptons } \\
Particle & Mass $\left(\mathrm{MeV} / c^{2}\right)^{3}$ & Charge (e) & $L_{e}$ & $L_{\mu}$ & $L_{\tau}$ \\
\hline$\nu_{e}$ & $<3 \times 10^{-6}$ & 0 & 1 & 0 & 0 & \\
$e$ & 0.511 & -1 & 1 & 0 & 0 & \\
$\nu_{\mu}$ & $<0.19$ & 0 & 0 & 1 & 0 & \\
$e$ & 0.511 & -1 & 0 & 1 & 0 & \\
$\nu_{\tau}$ & $<18.2$ & 0 & 0 & 0 & 1 & \\
$e$ & 0.511 & -1 & 0 & 0 & 1 & \\
\hline \multicolumn{7}{c}{ Quarks } \\
Particle & Mass $\left(\mathrm{GeV} / c^{2}\right)$ & Charge (e) & $S$ & $C$ & $B$ & $T$ \\
\hline $\mathrm{u}$ & $1-5 \times 10^{-3}$ & $2 / 3$ & 0 & 0 & 0 & 0 \\
$\mathrm{~d}$ & $3-9 \times 10^{-3}$ & $-1 / 3$ & 0 & 0 & 0 & 0 \\
$\mathrm{c}$ & $1.15-1.35$ & $2 / 3$ & 0 & 1 & 0 & 0 \\
$\mathrm{~s}$ & $75-170 \times 10^{-3}$ & $-1 / 3$ & -1 & 0 & 0 & 0 \\
$\mathrm{t}$ & $174.3 \pm 5.1$ & $2 / 3$ & 0 & 0 & 0 & 1 \\
$\mathrm{~b}$ & $4-4.4$ & $-1 / 3$ & 0 & 0 & -1 & 0 \\
\hline
\end{tabular}

Table 1.2: Properties of quarks and leptons.

the fermions, there exists an anti-particle that carries the opposite quantum numbers. Table 1.2 summarizes quark and lepton properties.

\subsubsection{Shortcomings of the Standard Model}

Despite its overwhelming success, the Standard Model cannot explain all observed phenomena. The most obvious shortcoming is that it does not attempt to explain gravity. But the Standard Model also fails to explain some subatomic behavior, although it being constructed to do so.

\footnotetext{
${ }^{3}$ Neutrino flavors do no have definite mass. See Sec. 1.1.1 for more discussion.
} 


\section{Neutrino Oscillations}

The existence of neutrino oscillations was proven at the turn of the $21^{\text {st }}$ century [1], after decades of hints [1, 2]. This is the phenomenon by which a neutrino of one flavor propagates and is later found to be another flavor. Neutrinos interact as flavor eigenstates $\left|\nu_{\alpha}\right\rangle$ but propagate as mass eigenstates $\left|\nu_{i}\right\rangle$. The flavor eigenstates are superpositions of mass eigenstates which are related by the PMNS matrix [3]:

$$
\left(\begin{array}{c}
\nu_{e} \\
\nu_{\mu} \\
\nu_{\tau}
\end{array}\right)=\left(\begin{array}{ccc}
U_{e 1} & U_{e 2} & U_{e 3} \\
U_{\mu 1} & U_{\mu 2} & U_{\mu 3} \\
U_{\tau 1} & U_{\tau 2} & U_{\tau 3}
\end{array}\right)\left(\begin{array}{c}
\nu_{1} \\
\nu_{2} \\
\nu_{3}
\end{array}\right)
$$

or expressed as three mixing angles and a phase

$$
U=\left(\begin{array}{ccc}
c_{12} c_{13} & s_{12} c_{13} & s_{13} e^{-i \delta} \\
-s_{12} c_{23}-c_{12} s_{23} s_{13} e^{i \delta} & c_{12} c_{23}-s_{12} s_{23} s_{13} e^{i \delta} & s_{23} c_{13} \\
s_{12} s_{23}-c_{12} c_{23} s_{13} e^{i \delta} & -c_{12} c_{23}-s_{12} c_{23} s_{13} e^{i \delta} & c_{23} c_{13}
\end{array}\right)
$$

where $c_{i j}=\cos \left(\theta_{i j}\right)$ and $s_{i j}=\sin \left(\theta_{i j}\right)$. The time evolution of the mass eigenstate state is given by the Schrödinger equation

$$
\left|\nu_{i}(t)\right\rangle=e^{-i E_{i} t}\left|\nu_{i}(0)\right\rangle
$$

where $E_{i}$ is the total energy of the neutrino. Combining Eq. 1.3 and 1.2 with the simplification of considering only two neutrino flavors, say the $\mu$ and $\tau$, yields the probability for oscillation from one flavor to another as

$$
P_{\mu \rightarrow \tau}=\sin ^{2}\left(2 \theta_{23}\right) \sin ^{2}\left(\frac{\Delta m_{23}^{2} L}{4 E}\right)
$$

where $\theta_{23}$ is an element of the PMNS matrix, $\Delta m_{23}=\left|m_{3}-m_{2}\right|$ is the difference in mass between eigenstates, $L$ is the distance of propagation, and $E$ is the energy of the neutrino.

The existence of oscillations requires that the neutrino masses are different and thus that they have non-zero mass. Yet neutrinos in the Standard Model do not have mass. Developing 
an understanding of neutrino interactions is rightfully one of the top priorities of the particle physics community, because it will surely reveal new physics.

\section{The EMC Effect}

In 1983, the European Muon Collaboration (EMC) reported measurements of the structure of the proton that exhibited new and, even today, not fully understood behavior [4]. The measurements of the structure function $F_{2}$, which describes the momenta of constituents of the proton and neutron, were made by scattering muons inelastically off iron and deuterium. The interesting and unexpected result was that $F_{2}$ was different in the two targets. This means that the quark structure of a nucleon is effectively modified inside a nucleus. The modifications observed were a suppression of quarks with very low momenta $\left(x_{b j}<0.1\right]^{4}$, an enhancement at slightly higher momenta $\left(0.1<x_{b j}<0.3\right)$, and a significant suppression again at moderate momenta $\left(0.3<x_{b j}<0.7\right)$. Because no physical mechanism could be used the explain the observation, it became known as the "EMC effect", after the collaboration that first reported it. Since the first observation of the EMC effect, there have been numerous precision measurements of the nuclear dependence of quark distributions, mostly through the use of deep inelastic electron or muon scattering. These have led to a deeper understanding of quantum chromodynamics (QCD) which describes the interactions among quarks and gluons in the nucleus. The regions at lower $x_{b j}$ are now understood to be due to interference between the scattering from different quarks/nucleons in the nucleus. The term "EMC effect" is now commonly used to refer only to the behavior at $0.3<x_{b j}<0.7$, which still is not understood.

\footnotetext{
${ }^{4}$ Bjorken $\mathrm{x}\left(x_{b j}\right)$, in simplified terms, is the fraction of a nucleon's momentum carried by the quark that participates in the scattering process.
} 


\subsection{Neutrino Scattering}

Neutrinos interact only weakly 5 . If the interaction is mediated by a $W^{ \pm}\left(Z^{0}\right)$ it is called a charged (neutral) current interaction. A charged current neutrino interaction has a charged lepton with matching lepton number in the final state. For $\nu_{\mu}$ scattering, there will be a $\mu^{-}$ in the final state. In neutral current interactions, the final state lepton is a neutrino that has the same lepton number as the incident neutrino. This dissertation presents an analysis of the charged current cross section and has negligible backgrounds from neutral current events, so only charged current interactions are described in this chapter. There are three dominant interaction channels for neutrino-nucleon scattering neutrino energies in the few-GeV range, which are characterized by the state of the struck nucleon after the interaction. These are:

- Quasi-elastic scattering - The neutrino scatters off the whole nucleon. No pions are produced.

- Resonance production - The interaction leaves the nucleon in an excited resonance state. The resonance most often decays into one nucleon and one pion.

- Deep inelastic scattering - The neutrino interacts with a quark in the nucleus. The ejected quark hadronizes, creating any number of hadronic final state particles. Often there are many pions produced.

Deep inelastic scattering (DIS) is somewhat arbitrarily defined as interactions with $Q^{2}>1(\mathrm{GeV} / c)^{2}$ and $W>2 \mathrm{GeV}$, and resonance scattering as interactions with pion production at smaller $Q^{2}$ or $W$ than the DIS limits. Neutrino scattering from nuclei is more complex, but is usually characterized in the same way, where quasi-elastic scattering refers to events where no pion is produced, resonance scattering produces a single pion, and deep inelastic scattering produces one or more pions with the kinematic limits given above.

\footnotetext{
${ }^{5}$ Weakly here means via the weak force.
} 


\subsubsection{Quasi-Elastic Scattering}

Charged current quasi-elastic scattering of a neutrino or antineutrino off a free nucleon is respectively

$$
\begin{aligned}
& \nu_{l}(k)+n(p) \rightarrow l^{-}\left(k^{\prime}\right)+p\left(p^{\prime}\right) \text { or } \\
& \bar{\nu}_{l}(k)+p(p) \rightarrow l^{+}\left(k^{\prime}\right)+n\left(p^{\prime}\right),
\end{aligned}
$$

where the lepton flavor $l$ is $e, \mu$ or $\tau$, and is the same on both sides. This is the dominant process for $E_{\nu}<2 \mathrm{GeV}$.

The differential cross section for this interaction can be written as

$$
\frac{d \sigma^{\nu, \bar{\nu}}}{d Q^{2}}=\frac{M_{p, n}^{2} G_{F}^{2} \cos ^{2}\left(\theta_{c}\right)}{8 \pi\left(1+\frac{Q^{2}}{M_{W}^{2}}\right)^{2} E_{\nu}^{2}}\left[A\left(Q^{2}\right) \pm B\left(Q^{2}\right) \frac{s-u}{M_{p, n}^{2}}+C\left(Q^{2}\right) \frac{(s-u)^{2}}{M_{p, n}^{4}}\right]
$$

where $\theta_{c}$ is the Cabibbo angle, $s$ and $u$ are Mandelstam variables, and the functions $A\left(Q^{2}\right)$, $B\left(Q^{2}\right)$ and $C\left(Q^{2}\right)$ are expressed in terms of the nucleon form factors [5]. The scalar and axial tensor form factors vanish if the hadronic current is time and charge invariant [6]. The induced pseudo-scalar form factor has a negligible effect because it is multiplied by $\left(m_{l} / M_{p, n}\right)^{2}$. The functions $A\left(Q^{2}\right), B\left(Q^{2}\right)$ and $C\left(Q^{2}\right)$ then take the form

$$
\begin{gathered}
A\left(Q^{2}\right)=\frac{m_{l}^{2}+Q^{2}}{M_{p, n}^{2}}\left[(1+\tau)\left|F_{A}\right|^{2}+(\tau-1)\left|F_{V}^{1}\right|^{2}+\tau\left|\xi_{p, n} F_{V}^{2}\right|^{2}(1-\tau)\right. \\
\left.+4 \tau F_{V}^{1} \xi_{p, n} F_{V}^{2}-\frac{m_{l}^{2}}{4 M_{p, n}^{2}}\left(\left(F_{V}^{1}+\xi_{p, n} F_{V}^{2}\right)^{2}+\left|F_{A}\right|^{2}\right)\right] \\
B\left(Q^{2}\right)=4 \tau\left(F_{V}^{1}+\xi_{p, n} F_{V}^{2}\right) F_{A}, \text { and } \\
C\left(Q^{2}\right)=\frac{1}{4}\left(\left|F_{A}\right|^{2}+\left|F_{V}^{1}\right|^{2}+\tau\left|\xi_{p, n} F_{V}^{2}\right|^{2}\right)
\end{gathered}
$$

where $\tau=\frac{Q^{2}}{4 M_{p, n}^{2}}$ again. The Nachtmann scaling variable $\xi_{p, n}=2(1+\sqrt{1+\tau})^{-1}$ relates the elastic to inelastic form factors [7]. The vector form factors $F_{V}^{1}$ and $F_{V}^{2}$ have been precisely measured using $e N$ scattering. Under the Conserved Vector Current (CVC) Hypothesis, the 
vector form factors obtained from charged lepton scattering can be applied to neutrino scattering [8].

\subsubsection{Resonance Production}

A baryon resonance $\left(\Delta\right.$ or $\left.N^{*}\right)$ is produced when a neutrino produces an excited state of the nucleon. The baryon resonance decays, so that the effective hadronic final state for charged current neutrino-induced resonance production is one nucleon and one or more mesons. For example, production of the $\Delta^{++}$resonance is

$$
\begin{aligned}
\nu+N \rightarrow l+\Delta^{++} & \\
\Delta^{++} & \rightarrow \pi^{+} p .
\end{aligned}
$$

Single pion production is roughly $1 / 3$ of the cross section for neutrinos in the energy range of $2 \mathrm{GeV}<E_{\nu}<7 \mathrm{GeV}$.

The Rein-Sehgal treatment of neutrino-induced baryon resonances [9] is to first calculate the cross section for a resonance with an infinitely narrow mass width:

$$
\frac{d \sigma}{d q^{2} d \nu}=\frac{G_{F}^{2}}{4 \pi^{2}}\left(\frac{-q^{2}}{Q^{2}}\right) \kappa\left(u^{2} \sigma_{L}+\nu^{2} \sigma_{R}+2 \mu \nu \sigma_{s}\right),
$$

where $G_{F}$ is the Fermi constant, $q$ is the 4-momentum transfer, $Q^{2}$ is the 3-momentum transfer squared, $\sigma_{L, R, s}$ are components of polarization of the mediating $W, u=\left(E+E^{\prime}+Q\right) / 2 E$, and $v=\left(E+E^{\prime}-Q\right) / 2 E$; the kinematic factor $\kappa=\left(M^{2}-M_{N} 2\right) / 2 M_{N}$, where where $M$ is the resonance mass and $M_{N}$ is the mass of the ground state nucleon. Then, the cross section for production of this hypothetical baryon is integrated over the appropriate width of the resonance $\Gamma$ in question using a Breit-Wigner factor:

$$
\delta(W-M) \rightarrow \frac{1}{2 \pi} \frac{\Gamma}{(W-M)^{2}+\Gamma^{2} / 4}
$$




\subsubsection{Deep Inelastic Scattering}

A neutrino scatters off a quark in a nucleon in deep inelastic scattering (DIS) [10]. The cross section depends on the energy of the incoming neutrino, the kinematics of the exchanged boson, and the quark structure of the struck nucleon. The kinematic factors involved are $Q^{2}$ (square of the four momentum transferred), $\nu$ (energy of the exchanged boson), $y$ (inelasticity or the fraction of the incoming energy carried by the outgoing hadronic system), and $x_{b j}$ (Bjorken scaling variable, or the fraction of the nucleon's momentum carried by the struck quark in the infinite momentum frame).

$$
\begin{aligned}
Q^{2} & =2 E_{\nu}\left(E_{\mu}-p_{\mu} \cos \left(\theta_{\mu}\right)\right) \\
\nu & =E_{\nu}-E_{\mu} \\
W & =\sqrt{M^{2}+2 M \nu-Q^{2}} \\
x & =\frac{Q^{2}}{2 M \nu} \\
y & =\nu / E_{\nu}
\end{aligned}
$$

The cross section can be written as

$$
\begin{aligned}
\frac{d^{2} \sigma^{\nu, \bar{\nu}}}{d x d y} & =\frac{G_{F}^{2} M_{n} E_{\nu}}{\pi\left(1+Q^{2} / M_{W, Z}^{2}\right)^{2}} \\
& {\left[\frac{y^{2}}{2} 2 x F_{1}\left(x, Q^{2}\right)+\left(1-y-\frac{x y M_{n}}{2 E_{\nu}}\right) F_{2}\left(x, Q^{2}\right) \pm y\left(1-\frac{y}{2}\right) x F_{3}\left(x, Q^{2}\right)\right], }
\end{aligned}
$$

where the $F_{3}$ term is added (subtracted) for $\nu(\bar{\nu})$. There are two other allowed structure functions, called $F_{4}$ and $F_{5}$, but they are multiplied by kinematic factors proportional to $\left(m_{l^{ \pm}}^{2} / M_{n} E_{\nu}\right)^{2}$ and $m_{l^{ \pm}}^{2} / M_{n} E_{\nu}$ respectively, which makes them negligible. The $F_{i}$ are dimensionless structure functions which describe the quark structure of the nucleon. 
The relevant structure functions for neutrino DIS at leading order are

$$
\begin{aligned}
F_{2}\left(x, Q^{2}\right) & =2 \sum_{q=u, d, \ldots}\left[x q\left(x, Q^{2}\right)+x \bar{q}\left(x, Q^{2}\right)\right], \\
x F_{3}\left(x, Q^{2}\right) & =2 \sum_{q=u, d, \ldots}\left[x q\left(x, Q^{2}\right)-x \bar{q}\left(x, Q^{2}\right)\right], \text { and } \\
2 x F_{1}\left(x, Q^{2}\right) & =F_{2}\left(x, Q^{2}\right) \frac{1+4 M^{2} x^{2} / Q^{2}}{1+R_{L}\left(x, Q^{2}\right)},
\end{aligned}
$$

where the $q\left(x, Q^{2}\right)$ are parton distribution functions (PDFs) interpreted as the probability that parton species $q$ has $x$ fraction of the nucleon's momentum when probed at $Q^{2}$. Note that $x F_{3}$ should have no or very small contribution from sea quarks, under the assumption that they are created in $q \bar{q}$ pairs. $F_{1}$ is related to $F_{2}$ by the longitudinal structure function $R_{L}$.

\subsection{Nuclear Effects}

Neutrino experiments are frequently forced to employ heavy materials in order to obtain the statistics necessary for precise measurements, because the cross sections involved are so small. Water, carbon, iron, lead, and, more recently, argon are the most commonly used nuclear materials in neutrino detectors. The use of nuclear targets complicates the observation of neutrino interactions in two ways. Nuclear structure and correlations among the nucleons within the nucleus can directly affect the event rate or kinematics of neutrino interaction processes. In addition, the final state hadrons produced by the neutrino interaction can interact with the residual nucleus in various ways, processes collectively referred to as final state interactions (FSI). These nuclear effects are poorly understood in neutrino scattering and contribute considerably to uncertainties in neutrino oscillation measurements. There are no accurate measurements of these nuclear effects using neutrinos in the few-GeV energy regime. 


\subsubsection{Primary Neutrino Interaction}

Nucleons are bound in a nucleus by the strong force. The interaction probabilities for bound nucleons differ from those for free nucleons due to the QCD physics that binds the nucleus [11, [12]. At present, there is no complete QCD description of a nucleon, let alone of a nucleus. Treatment of the nuclear modifications to interaction probabilities is a patchwork of theories and parameterizations to data. Modifications of cross sections of a bound nucleon compared to a free nucleon have been extensively, though not exhaustively, measured in charged lepton scattering. However, neutrinos are sensitive to different aspects of quark structure, so these charged lepton measurements must be translated to the neutrino sector.

The mechanisms that give rise to a modified cross section are organized into ranges of the scaling variable $x_{b j}$ (defined in Eq. 1.13). The dominant nuclear modifications are in the following $x_{b j}$ regimes 6 ;

- Shadowing $\left(x_{b j} \lesssim 0.1\right)-$ Suppression of the cross section due to interactions with hadronic fluctuations of a $W$ and the target.

- The EMC effect $\left(0.3 \lesssim x_{b j} \lesssim 0.7\right)-$ A phenomenon with no accepted theoretical explanation which appears to be smearing of quark distributions towards lower momenta.

- Fermi motion $\left(0.7 \lesssim x_{b j}\right)$ - Momentum of nucleons in the nucleus smears quark momentum distributions.

One other nuclear modification is considered which is presently of great interest to the neutrino community: an enhancement of the transverse quasi-elastic cross section due to nucleonnucleon correlations.

\footnotetext{
${ }^{6}$ These boundaries on $x_{b j}$ are approximate and can depend on the process or nuclear target.
} 


\section{Shadowing}

Shadowing is a reduction in the cross section due to destructive interference of interactions with multiple nucleons in a nucleus that occurs for $x_{b j} \lesssim 0.1$ [13, 14, 15]. Each nucleon is shielded from the force-mediating vector boson by the other nucleons in the nucleus. Before interacting with a particular nucleon, the vector boson must survive a journey through the nucleus before colliding with said nucleon. In the case of neutrino scattering, the $W$ can interact during hadronic fluctuations. The partial conservation of axial current (PCAC) leads to the Adler relation, which describes the axial-vector hadronic states to which the $W$ may fluctuate. In contrast to the vector meson dominance (VMD) observed in the hadronic nature of the $\gamma$, there are many hadronic states that make significant contributions to the hadronic behavior exhibited by a $W$. The axial current has contributions from $W$ fluctuations to the $a_{1}$ meson, $p-\pi$ system, and other states.

Nuclear shadowing is fairly well understood and has been successfully measured with charged lepton scattering. However, there are two important expected differences in the shadowing of neutrino interactions. The first is that the shadowing of sea quarks differs from valence quarks. Neutrinos, unlike charged leptons, are directly sensitive to the number of valence quarks through the structure function $F_{3}$. Shadowing is different for axial and vector currents, and only neutrinos are sensitive to the axial structure. This difference is most pronounced at low $Q^{2}$, since the vector current vanishes at $Q^{2} \rightarrow 0 \mathrm{GeV} / c^{2}$ (because it is conserved) and the axial does not.

\section{EMC Effect}

The EMC effect is a $x_{b j}$-dependent modification in the cross section in the approximate range $0.3 \lesssim x_{b j} \lesssim 0.7$. The observation is a suppression of the structure function $F_{2}$ that grows at larger $x_{b j}$. There is no accepted explanation for this behavior, and the EMC effect remains an interesting experimental and theoretical topic. 
The "antishadowing" region at $0.1 \lesssim x_{b j} \lesssim 0.2$ is so-named because it was believed to be caused by constructive interference in multiple scattering through a mechanism very similar to shadowing. However, the coherence time at such values of $x_{b j}$ is too small for such long range N-N correlations. The "antishadowing" effect is an enhancement in the bound nucleon cross section which may instead be related to the EMC effect.

One of the many models suggested to explain the EMC effect is the existence of multiquark clusters, in which the nucleus contains groups of $3 N$ quarks [16]. While a quark in a free nucleon cannot have $x_{b j}>1$, a multiquark cluster can. The existence of very high momentum states would in turn suppress $F_{2}$ at lower values of $x_{b j}$. Multiquark models became popular shortly after the original EMC publication, and they may come into vogue again with recent measurements that show a correlation of structure functions in the EMC range to those at $x_{b j}>1$ [17]. This explanation is by no means widely accepted; it is given here merely as an example.

\section{Fermi Motion and the Fermi Gas Model}

Fermi motion is the momentum that nucleons have relative to the nuclear center of mass. It is the dominant nuclear effect at $\left(0.7 \lesssim x_{b j}\right)$. A common model of the nucleus that explains this phenomenon is the relativistic Fermi gas (RFG) [18, 19]. In this model, the nucleus is made of two systems of free nucleons (one each for neutrons and protons) that obey Fermi statistics confined in a potential well. The potential felt by each nucleon is due to the superposition of the forces on it from all other nucleons. The nucleons sit in a well potential with walls at the nucleus radius. When the nucleus is in the ground state, the nucleons fill the lowest energy levels. The Fermi momentum $p_{F}$ is the largest momentum of a nucleon for the nucleus in a ground state. The nucleons have a flat momentum distribution that goes to zero above $p_{F}$.

There are other two nuclear effects explained with RFG. Pauli blocking within the gas significantly suppresses the cross section at low $Q^{2}$. The nuclear binding energy $E_{b}$ imposes 
a minimum energy that must be imparted to the hadronic system for a nucleon to be removed from the nucleus or be excited to a higher energy bound state, which suppresses some cross sections and changes the energy of the observed hadronic system.

Although it is the strong force that binds nucleons in a nucleus, the binding in this model is rather weak. The nucleons in the Fermi gas model are non-interacting; the nucleus is an incoherent sum of nucleons. This aspect of the model is not realistic. Bodek and Ritchie introduced an extension to the RFG that takes into account N-N correlations [20]. The BodekRitchie model adds high momentum tails to the nucleon momentum distribution.

\section{Transverse Enhancement}

The transverse $e A$ quasi-elastic cross section is enhanced for bound nucleons. Although transverse enhancement has been known and modeled in charged lepton scattering for more than a decade, the neutrino community is only recently mobilizing to account for the effect in simulations and analyses [21]. The most popular theory for the enhancement is currently meson exchange currents (MEC). In the theory, the probe can interact with hadronic currents that mediate forces between correlated nucleons. By far, the most frequently correlated pair is $n p$ [22]. The size of the effect depends on the nucleus.

Accounting for transverse enhancement in the quasi-elastic channel significantly reduces tension in an infamous discrepancy between measurements by MiniBooNE near $2 \mathrm{GeV}$ and

measurements by NOMAD near $5 \mathrm{GeV}[21]$. This underscores how imperative it is for the neutrino community to treat nuclear effects with more rigor.

\subsubsection{Final State Interactions}

Hadrons produced in neutrino-nucleus interactions begin in the nuclear environment. As they propagate through the nucleus, they are subject to possible hadronic interactions with it. These are known as final state interactions (FSI). FSI cause the observed hadronic system 
to differ from the system produced by the neutrino interaction. The severity of the problem depends on the nature of the particular final state, detector, and analysis techniques.

Consider the effects of FSI in neutrino single pion production. Pions of energies produced by few-GeV neutrino interactions are subject to four types of FSI [23]:

- Elastic Scattering - The pion scatters and the nucleus remains in the ground state.

- Inelastic Scattering - The pion scatters and the nucleus is left in an excited state, or a nucleon may be ejected from the nucleus.

- Absorption - The pion can be absorbed, leaving no pion in the final state.

- Charge Exchange - The pion changes charge, most often charged $\leftrightarrow$ neutral.

Some of these interactions change the number and/or type of final state hadrons, which may result in misidentification of a signal event. If any of these processes occurs, the neutrino energy will be reconstructed incorrectly. Proper reconstruction of the neutrino energy is crucial in the measurement of neutrino oscillations, and FSI present a serious difficulty.

The probability that FSI occur is very high because the cross section of hadronic interactions are so large. The total $\pi A$ cross section can be larger than 1 barn 7 Roughly half of all few-GeV $\nu F e$ interactions undergo some FSI [24].

\footnotetext{
${ }^{7}$ Pion-nucleus cross sections seem especially large to a neutrino physicist who is familiar with interaction probabilities that are $\sim 14$ orders of magnitude smaller!
} 


\section{Chapter 2}

\section{Apparatus}

MINERvA (Main INjector Experiment $\nu$-A) is a neutrino scattering experiment at Fermi National Accelerator Laboratory (FNAL) in the NuMI beam. NuMI delivers an intense flux of neutrinos or antineutrinos, created by the decay of mesons produced in $p \mathrm{C}$ collisions at $120 \mathrm{GeV} / c$. The MINERvA detector employs fine-grained polystyrene scintillator for tracking and calorimetry. In addition to the active scintillator target, the detector contains passive nuclear targets of carbon, iron, lead, water, and liquid helium. The MINOS near detector sits downstream of MINERvA and is the muon spectrometer.

\subsection{NuMI Neutrino Beam}

The NuMI (Neutrinos at the Main Injector) beamline is a conventional accelerator neutrino source at Fermilab [25]. NuMI can provide an intense flux of either mostly muon neutrinos or mostly muon antineutrinos to short- and long-baseline neutrino experiments MINERvA, MINOS, ArgoNeuT, and NOvA [26, 27, 28, 29].

$120 \mathrm{GeV} / c$ protons from the Main Injector bombard a graphite target which produces many mesons, mostly pions and kaons. Two pulsed magnetic horns focus positive (negative) mesons which will decay to produce a predominately $\nu_{\mu}\left(\bar{\nu}_{\mu}\right)$ beam. The beam propagates through a beam dump and earth to absorb the undecayed mesons and muons. The NuMI beam is directed into the Earth at a pitch of $58 \mathrm{mrad}$, so that the center of the neutrino flux penetrates the MINOS far detector in Soudan, MN. A near detector cavern for short baseline neutrino experiments is $\sim 1 \mathrm{~km}$ downstream of the NuMI target and $100 \mathrm{~m}$ underground. The flux of neutrinos along 
the focusing axis have energies $1-50 \mathrm{GeV}$. A schematic of the NuMI beamline is shown in Fig. 2.1.

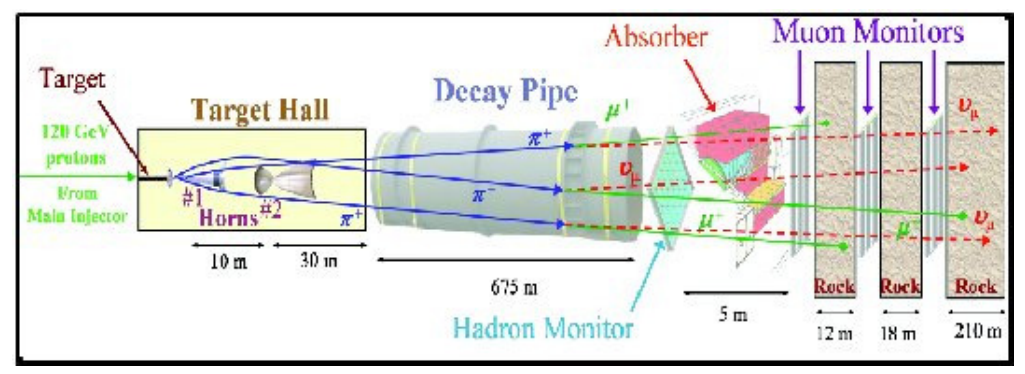

Figure 2.1: A schematic drawing of the NuMI beamline. Reprinted from [30], Copyright (2005) with permission from $\mathrm{R}$. Zwaska.

\subsubsection{Proton Delivery}

The Booster accelerator delivers $8 \mathrm{GeV}$ protons to the Main Injector ring, which accelerates the protons to $120 \mathrm{GeV} / c$ [31]. The protons are accelerated with $53 \mathrm{MHz}$ radio frequency (rf) waves. This rf signal creates 558 potential wells, which are called rf buckets, around the $3.3 \mathrm{~km}$ circumference of the accelerator ring. Using the rf signal, the protons are grouped into six batches. The Booster has the ability to inject additional batches on top of those already stored in the Main Injector. Protons from the Main Injector are extracted to NuMI via a single turn extraction with a repetition rate of $0.53 \mathrm{~Hz}$. Typically, when the Tevatron was operational, the final five batches were extracted to NuMI. After the Tevatron was decommissioned on September 30, 2011, all six batches were extracted to NuMI. A beam spill containing all six batches normally lasts $10 \mu$ s and delivers $\sim 42 \times 10^{12}$ protons per pulse (PPP); a typical spill of five batches lasts $8.4 \mu$ s and delivers $\sim 35 \times 10^{12}$ PPP. Roughly $15 \mathrm{~cm}$ prior to striking the target, the proton beam passes through a toroid which measures the number of protons, and the beam profile is monitored to ensure a proper beam spot. 


\subsubsection{NuMI Target and Horns}

A $120 \mathrm{GeV} / c$ proton beam is collimated to $\sim 1 \mathrm{~mm}$ immediately before striking the NuMI target by a baffle. This ensures that stray protons do not bombard the support structure and that the beam is not overly focused, which would damage the target. The target is comprised of 47 graphite fins, which are mounted to steel pipes along the beam direction (c.f. Fig. 2.2). The steel pipes provide water cooling. The fins are narrow $(6.4 \mathrm{~mm}$ wide and $15 \mathrm{~mm}$ tall) so that final state hadrons produced by $120 \mathrm{GeV} / c p \mathrm{C}$ collisions are not likely to reinteract in the target. Each fin is $20 \mathrm{~mm}$ long, giving the target a total length of $\sim 940 \mathrm{~mm}$, which corresponds to 2 interaction lengths [32]. The target is electrically isolated, so that the emission of charged particles from a $48^{\text {th }}$ fin can be monitored to understand the shape and intensity of the incoming proton beam; this use of the $48^{\text {th }}$ fin is called a "Budal monitor" [33]. Of the proton interactions which eventually produce a neutrino passing through detectors in the MINOS detector cavern, $89 \%$ come from carbon (in either the proper target or the Budal monitor); the remaining interactions occur in support structures or ambient gas [34].

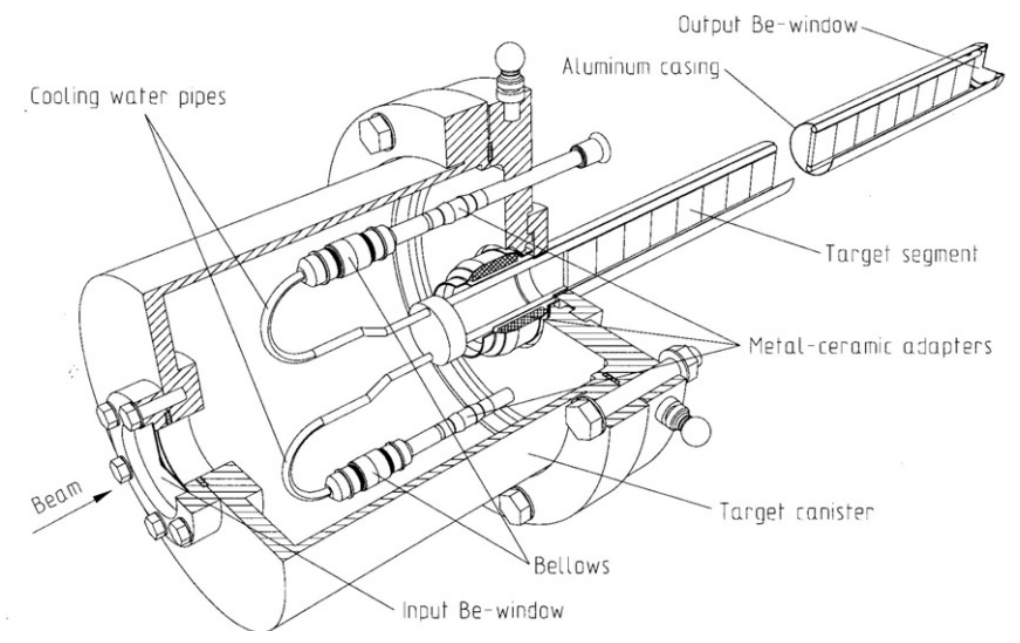

Figure 2.2: The NuMI target. Reprinted from [35], Copyright (2002) with permission from Fermi National Accelerator Laboratory.

Hadrons produced in the target are focused by two $3 \mathrm{~m}$ long magnetic horns situated downstream of the target, as shown in Fig. 2.3 . The horns are pulsed with a current of $+(-) 185 \mathrm{kA}$ 


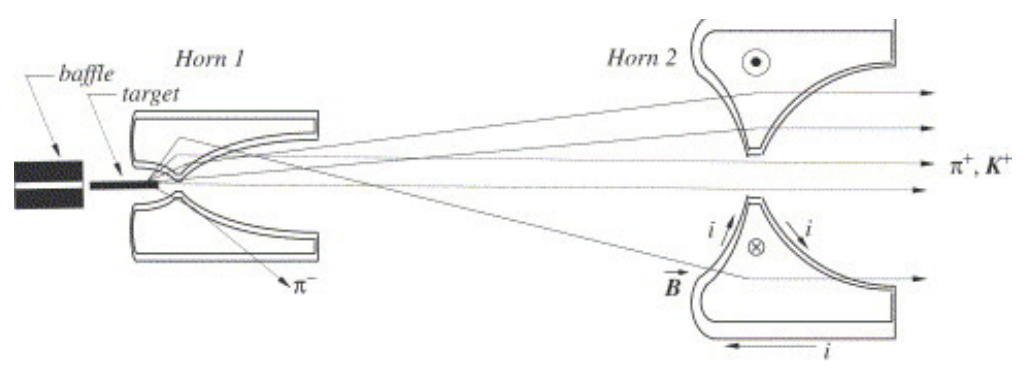

Figure 2.3: Schematic showing positions of the NuMI baffle, target, and horns. Reprinted from [32], Copyright (2006) with permission from Elsevier.

\begin{tabular}{lc}
\hline Decay Mode & Fraction \\
\hline$\pi^{+} \rightarrow \mu^{+} \nu_{\mu}$ & $99.99 \%$ \\
$\pi^{+} \rightarrow \mu^{+} \nu_{\mu} \gamma$ & $2.00 \times 10^{-4}$ \\
$\pi^{+} \rightarrow \mathrm{e}^{+} \nu_{e}$ & $1.23 \times 10^{-4}$ \\
$\mathrm{~K}^{+} \rightarrow \mu^{+} \nu_{\mu}$ & $63.55 \%$ \\
$\mathrm{~K}^{+} \rightarrow \pi^{0} \mathrm{e}^{+} \nu_{e}$ & $5.07 \%$ \\
$\mathrm{~K}^{+} \rightarrow \pi^{0} \mu^{+} \nu_{\mu}$ & $3.35 \%$ \\
\hline
\end{tabular}

Table 2.1: Decay modes of $\pi^{+} / \mathrm{K}^{+}$(charge conjugate for $\pi^{-} / \mathrm{K}^{-}$) resulting in neutrino relevant in the NuMI beam [36].

to bend positive (negative) $\pi$ 's and $K$ 's towards the primary proton beam's path. The current in the horns can be varied to support special studies of the beamline. The target can be moved up to $2.5 \mathrm{~m}$ upstream relative to the horns, which changes the momentum spectra of the focused particles, thereby allowing for a variable energy spectrum. Moving the target away from the horns focuses mesons with a higher energy, resulting in a higher energy beam.

Of the mesons whose decay will contribute to the neutrino flux in the MINOS detector cavern, $\sim 97 \%$ are $\pi$ 's and the rest are $K$ 's [34]. Focusing $\pi^{+} / \mathrm{K}^{+}\left(\pi^{-} / \mathrm{K}^{-}\right)$results in a beam of predominately $\nu_{\mu}\left(\bar{\nu}_{\mu}\right)$ through the decay channels listed in Table 2.1 .

\subsubsection{Decay and Detector Halls}

Secondary pions and kaons must be allowed to decay to produce a neutrino beam. This is accomplished in a cylindrical decay pipe which is $2 \mathrm{~m}$ wide, $675 \mathrm{~m}$ long, and held at a vacuum of $\sim 1$ Torr. The upstream end of the pipe is $50 \mathrm{~m}$ downstream of the target, giving a total of 
$725 \mathrm{~m}$ for the secondary beam particles to decay. NuMI pions have decay lengths ranging from roughly $0.1-5 \mathrm{~km}$ in accordance with their energy range $2-60 \mathrm{GeV}$.

Secondary hadrons and non-interacting primary protons are removed from the beam at the end of the decay pipe by a hadron absorber made of aluminum, steel, and concrete. A $1 \times 1 \mathrm{~m}^{2}$ hadron monitor records the position and intensity of the beam where the decay pipe meets the hadron absorber. Only neutrinos and muons are left in the beam at this point.

The beam passes through through $240 \mathrm{~m}$ of dolomite prior to entering the MINOS near detector cavern, which is enough material to filter all muons from the beam. The neutrino component of the beam is unaffected because of the extreme rarity of a neutrino interacting with the rock. Three muon monitors made of $2 \times 2 \mathrm{~m}^{2}$ arrays of ionization chambers, which record a pulse height proportional to the number of throughput muons, are installed in alcoves whose distance to the decay pipe varies. The amount of material between the point of meson decay and a muon monitor dictates the minimum momentum a muon must have had when born by pion decay. Since each muon monitor has a different muon momentum threshold, comparing rates among them yields information about kinematics of the pions that decay and thus of the neutrino beam. A fourth muon monitor was installed in the summer of 2013 in order to improve sensitivity to higher momentum muons which are more relevant in the medium energy beam.

Figure 2.4 shows the NuMI absorber region.

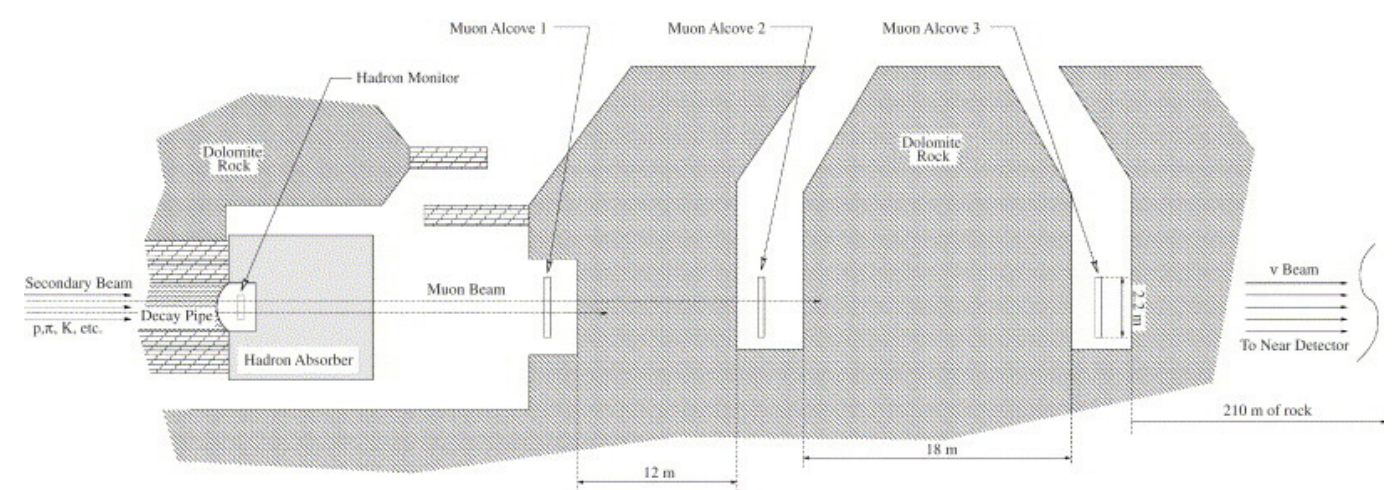

Figure 2.4: The NuMI secondary absorbers and monitors. Reprinted from [32], Copyright (2006) with permission from Elsevier. 


\subsection{The MINERvA Detector}

The MINERvA detector is designed to measure inclusive and exclusive neutrino-nucleus interaction channels for neutrino energies from $1-50 \mathrm{GeV}$. The major detector regions of MINERvA are show in Fig. 2.5. The hexagonal main core of the detector is approximately $5 \mathrm{~m}$ long and has inner and outer regions. The inner detector (ID) is longitudinally organized into four subdetectors: the nuclear targets region, the fully active tracking region, downstream electromagnetic calorimetry (ECAL), and downstream hadronic calorimetry (HCAL). The outer detector (OD) is a shell of hadronic calorimetry which surrounds and physically supports the ID. It serves both to contain particles produced by events in the detector and shield from particles produced by neutrino interactions outside of the detector. One ID module is mounted in the center of one OD frame, as shown in Fig. 2.6. The MINOS near detector sits downstream of MINERvA and serves as a toroidal muon spectrometer.

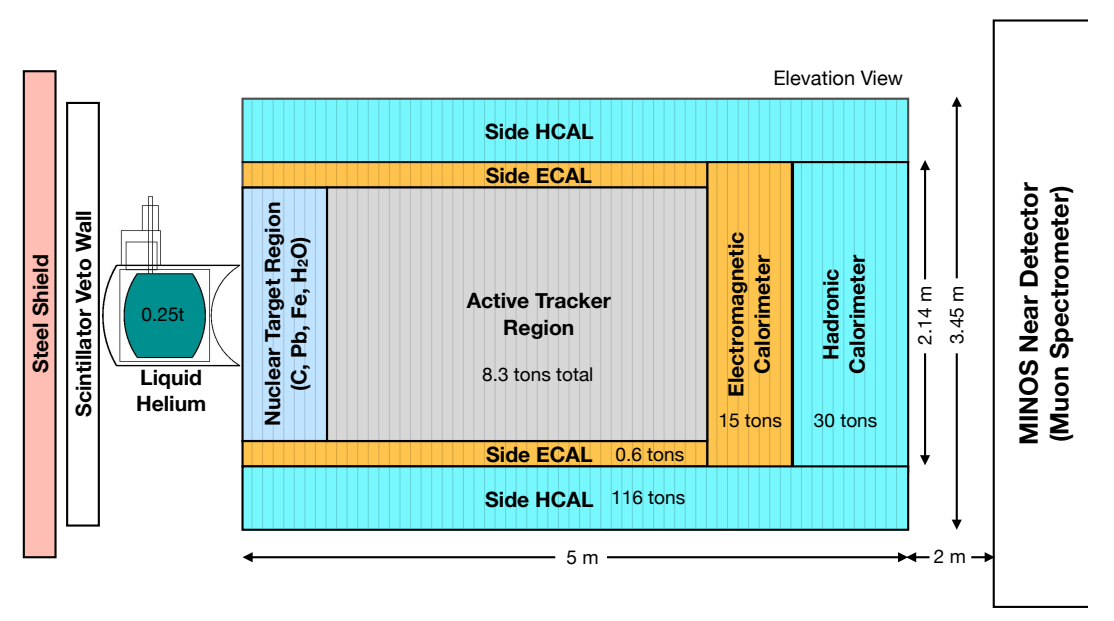

Figure 2.5: Major regions of the MINERvA detector.

\section{MINERvA Coordinate System}

MINERvA uses a right-handed coordinate system. The z-axis runs through center of the detector in the general direction of the neutrino beam. The detector, unlike the beam, is parallel 


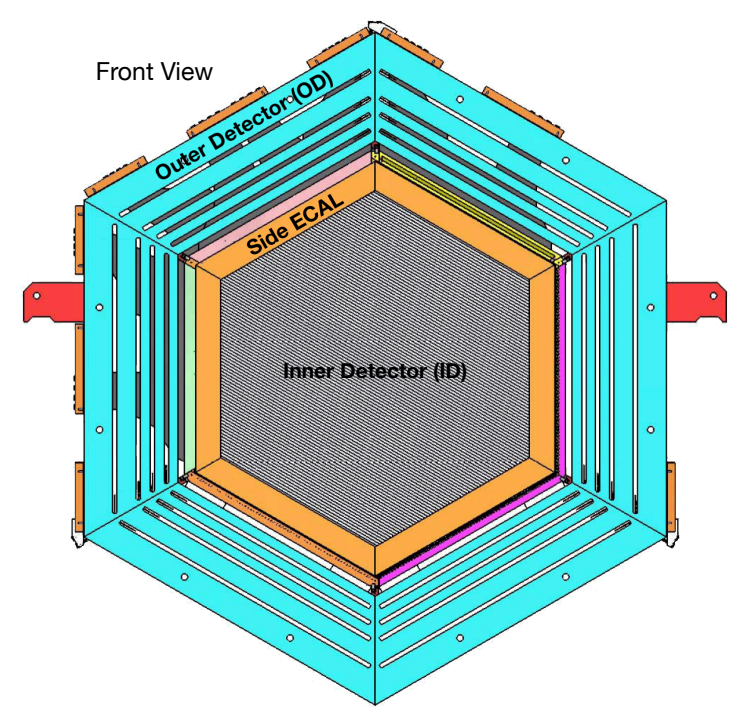

Figure 2.6: An inner detector tracking module mounted in an outer detector frame.

with the surface of the Earth 1 . The y-axis is up, away from the Earth. The x-axis is to the left from the perspective of the beam.

\subsubsection{Upstream Region}

Upstream of the main detector, there is a veto wall of iron slabs and scintillator paddles used to reduce and identify particles entering the front face of the detector. The paddles tag muons as having been produced upstream of the central MINERvA detector and helium target. The steel slabs can stop low energy hadrons or induce them to shower so that they might be detected by the paddles. A liquid helium target encased in a cryogenic vessel sits between the veto wall and the main detector. This target will be used as the "light" nuclear target in future analyses of nuclear dependence. The veto wall and helium target have yet to be used for physics analysis and are not described further here.

\footnotetext{
${ }^{1}$ Recall that the NuMI beam is directed into the Earth at pitch of $58 \mathrm{mrad}$.
} 


\begin{tabular}{c|ccc}
\hline Subdetector & Module Type & Module Numbers & N Scint. Planes \\
\hline Nuclear Targets & 22 tracking, 5 passive & $-5-22^{3}$ & 44 \\
Tracking Region & 62 tracking modules & $23-84$ & 114 \\
ECAL & 10 ECAL & $85-94$ & 20 \\
HCAL & 20 HCAL & $95-114$ & 20 \\
\hline
\end{tabular}

Table 2.2: Modular composition of subdetectors in the inner detector.

\subsubsection{Inner Detector}

The ID is a regular hexagon of apothem ${ }^{2} 1.07 \mathrm{~m}$ with a vertical major axis composed of 120 modules stacked longitudinally, numbered -5-114. There are tracking, ECAL, HCAL, and passive target modules. Except for the passive target modules, each module type employs scintillator as the active element. The arrangement of modules into subdetectors is summarized in Table 2.2.

\section{Scintillator Planes}

The active element in MINERvA is polystyrene, a solid plastic scintillator which is described further in Sec. 2.2.4. ID planes are made of triangular scintillator strips which are $33 \mathrm{~mm}$ wide and $17 \mathrm{~mm}$ high. The length of each strip varies based on its placement within the hegaxonal plane. A scintillator plane is made of 127 strips glued together with 3M-DP190 translucent epoxy, as shown in Fig. 2.7. Planes are made light tight by gluing on sheets of Lexan with 3M-DP190 gray epoxy. Remaining light leaks are sealed with black PVC electrical tape. The areal density of an assembled plane $\left(2.024 \mathrm{~g} / \mathrm{cm}^{2}\right)$ differs significantly from pure polystyrene $\left(1.65 \mathrm{~g} / \mathrm{cm}^{2}\right)$ because of the epoxy, Lexan, and tape. A summary of the density of and number of nucleons in a plane is shown in Table 2.3, this is broken down by nucleus in Table 2.4

\footnotetext{
${ }^{2}$ An apothem of a regular hexagon is the shortest distance from the center of the hexagon to an edge.

${ }^{3}$ One of the passive target modules (Target 3 ) occupies the space of two modules. So the nuclear targets region occupies the space of 28 modules.
} 


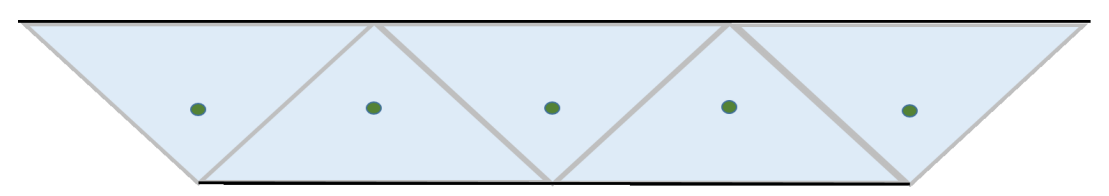

Figure 2.7: Cross sectional view of triangular scintillator strips in a plane. 127 Strips are glued together and wrapped in Lexan to make one scintillator plane.

\begin{tabular}{cccccc}
\hline Areal Mass $\left(\mathrm{g} / \mathrm{cm}^{2}\right)$ & Fiducial Area $\left(\mathrm{cm}^{2}\right)$ & Mass $(\mathrm{kg})$ & N Protons & N Neutrons & N Nucleons \\
\hline $2.02 \mathrm{e}+00$ & $2.50 \mathrm{e}+04$ & $5.07 \mathrm{e}+01$ & $1.63 \mathrm{e}+28$ & $1.42 \mathrm{e}+28$ & $3.05 \mathrm{e}+28$ \\
\hline
\end{tabular}

Table 2.3: Density and target number of a scintillator plane, assuming a fiducial area with an $85 \mathrm{~cm}$ apothem.

There are three possible orientations for the strips in the hexagon. If stacked horizontally, so that the length runs parallel to the $\mathrm{y}$-axis, each strip corresponds to a different $\mathrm{x}$ coordinate. This orientation is called the "X-view". The U- and V-views result from rotations in the XYplane by $+60^{\circ}$ and $-60^{\circ}$, respectively. These three views provide a stereoscopic 3D image of interactions in the detector. The use of three views gives redundant measurements of the two orthogonal coordinates $\mathrm{X}$ and $\mathrm{Y}$, which can be used to resolve ambiguity in the formation of 3D tracks.

A $0.2 \mathrm{~cm}$ thick hexagonal lead collar covers the outermost $15 \mathrm{~cm}$ of each plane's upstream face. This "side ECAL" stimulates electromagnetic showers and thus reduces the amount of energy leaving the ID.

\begin{tabular}{c|cccccc}
\hline Element & \% of Plane's Mass & Mass $(\mathrm{kg})$ & N Atoms & N Protons & N Neutrons & N Nucleons \\
\hline Carbon & 87.62 & $4.44 \mathrm{e}+01$ & $2.23 \mathrm{e}+27$ & $1.34 \mathrm{e}+28$ & $1.34 \mathrm{e}+28$ & $2.67 \mathrm{e}+28$ \\
Hydrogen & 7.42 & $3.76 \mathrm{e}+00$ & $2.25 \mathrm{e}+27$ & $2.25 \mathrm{e}+27$ & $1.78 \mathrm{e}+25$ & $2.26 \mathrm{e}+27$ \\
Oxygen & 3.18 & $1.61 \mathrm{e}+00$ & $6.06 \mathrm{e}+25$ & $4.85 \mathrm{e}+26$ & $4.85 \mathrm{e}+26$ & $9.70 \mathrm{e}+26$ \\
Aluminum & 0.26 & $1.32 \mathrm{e}-01$ & $2.94 \mathrm{e}+24$ & $3.82 \mathrm{e}+25$ & $4.11 \mathrm{e}+25$ & $7.93 \mathrm{e}+25$ \\
Titanium & 0.69 & $3.50 \mathrm{e}-01$ & $4.40 \mathrm{e}+24$ & $9.67 \mathrm{e}+25$ & $1.14 \mathrm{e}+26$ & $2.10 \mathrm{e}+26$ \\
Silicon & 0.27 & $1.37 \mathrm{e}-01$ & $2.93 \mathrm{e}+24$ & $4.11 \mathrm{e}+25$ & $4.13 \mathrm{e}+25$ & $8.24 \mathrm{e}+25$ \\
Chlorine & 0.55 & $2.79 \mathrm{e}-01$ & $4.73 \mathrm{e}+24$ & $8.05 \mathrm{e}+25$ & $8.73 \mathrm{e}+25$ & $1.68 \mathrm{e}+26$ \\
\hline
\end{tabular}

Table 2.4: Nuclear composition of a scintillator plane, assuming a fiducial area with a $85 \mathrm{~cm}$ apothem. 


\section{Tracking Modules}

A tracking module contains two scintillator planes in either the UX or VX orientations. Modules are stacked with alternating orientations yielding a pattern of UX/VX planes.

\section{Nuclear Targets}

The nuclear targets region contains 22 tracking modules and 5 solid passive targets. There are four tracking modules between targets, which is adequate for reconstructing tracks and showers. A schematic of the nuclear targets region is shown in Fig. 2.8 .

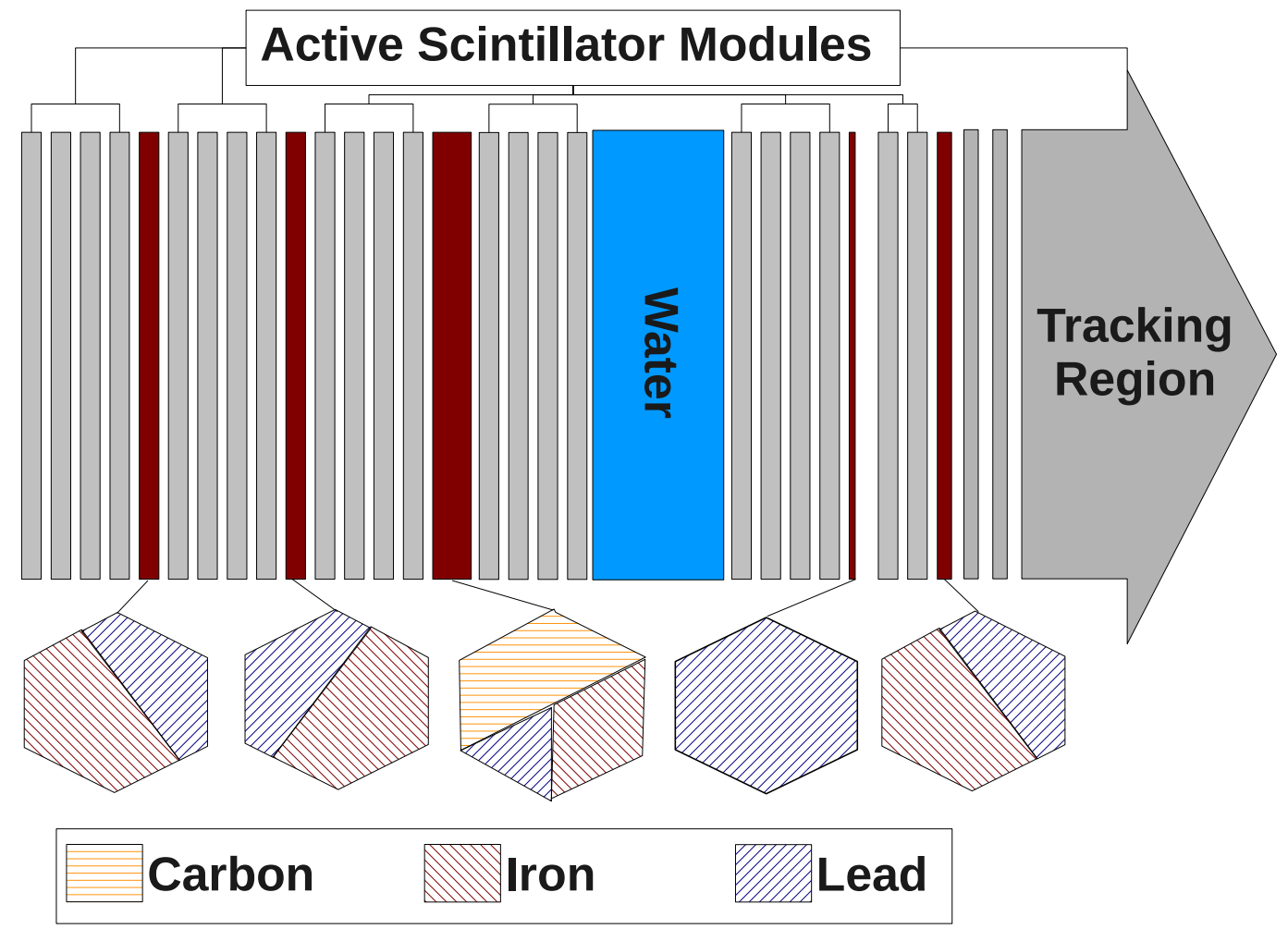

Figure 2.8: Schematic of the nuclear targets of MINERvA. Not shown is the liquid helium cryogenic vessel which sits just upstream (left) of the nuclear target region. The tracking region of the MINERvA detector is immediately downstream (right) of the nuclear target region.

Passive targets are numbered upstream to downstream 1-5. The targets are built out of transverse segments of carbon, iron, and lead. The mass of each nucleus is spread around the detector in both the longitudinal and transverse direction to reduce systematic errors from both 
the event rate and the development of showers originating in upstream targets. Thicker targets are the most upstream, so that they interfere with fewer final state products from interactions in other targets.

Targets 1, 2, and 5 contain iron and lead, which are divided diagonally on a $20.5 \mathrm{~cm}$ offset from the center of the hexagon. Target 3 is made of carbon, iron, and lead; which occupy $\frac{1}{2}, \frac{1}{3}$, and $\frac{1}{6}$ of the area of the target, respectively. Target 4 contains only lead. Each target has some unique features.

- Target 1 - Being the most upstream target makes Target 1 difficult to analyze. The primary difficulty is the small number of upstream tracking planes which can be used to identify particles produced outside of MINERvA.

- Target 2 - The orientation of iron and lead is flipped horizontally from that of Target 1.

- Target 3 - The carbon slice is three times as thick as the iron and lead, which are the same thickness as the iron and lead in Targets 1 and 2. All materials are flush at the upstream end, so that there is an air gap downstream of the iron and lead.

- Target 4 - Target 4 is much thinner than the other targets. It is intended to induce electromagnetic interactions of particles from the upstream targets before they enter the low $\mathrm{Z}$ tracking region. This both improves the containment of events in the upstream targets and reduces the noise from $n$ 's, $\gamma^{\prime}$ 's, and $\pi^{0}$ 's which would confuse event selection in the tracking region.

- Target 5 - Target 5 has the same shape as Target 1 but is half the thickness. As the large, fully active tracking region is immediately downstream of this target, tracking is a bit more precise than in other targets.

There is an air gap between Targets 3, 4, and 5 and the first scintillator plane downstream of them, which is beneficial for reconstruction because it allows for greater spatial separation of low energy hadronic final state particles. 


\section{ECAL}

ECAL modules are similar to tracking modules, except that a $0.2 \mathrm{~cm}$ lead sheet covers the entire downstream edge of each plane. The module just upstream of the ECAL region is a hybrid of tracking and ECAL modules which contains a lead collar on the upstream plane but a full lead sheet on the downstream plane. Therefore there is a lead sheet upstream of each tracking plane in the ECAL.

The lead in the ECAL stimulates electromagnetic showers. This helps to contain energy from photons, electrons, and pions which otherwise might exit the rear of the detector. The granularity of scintillator in the ECAL is just as fine as in the dedicated tracking region, which is easily sufficient to track charged particles.

\section{HCAL}

HCAL modules contain one scintillator plane and a $2.54 \mathrm{~cm}$ thick hexagonal steel plane. The scintillator plane is on the downstream end of each module. The planes in the HCAL have orientation pattern $\mathrm{Fe}|\mathrm{X} \mathrm{Fe}| \mathrm{V} \mathrm{Fe}|\mathrm{X} \mathrm{Fe}| \mathrm{U}$.

\subsubsection{Outer Detector}

The OD is a steel hexagonal shell with inner apothem $1.168 \mathrm{~m}$ and outer apothem $1.727 \mathrm{~m}$. All steel frames are $3.49 \mathrm{~cm}$ thick except for frames surrounding the thicker downstream HCAL where they are $3.81 \mathrm{~cm}$ thick. Each frame has four slots which hold a pair of $2.54 \times 2.54 \mathrm{~cm}^{2}$ rectangular scintillator strips for calorimetry and crude tracking. The distances from the inside edge of an OD frame to each pair of strips is $6.35,15.24,23.50$, and $37.47 \mathrm{~cm}$. The pairs are joined longitudinally so that each strip is roughly in line with one of the ID scintillator planes supported by the frame. 


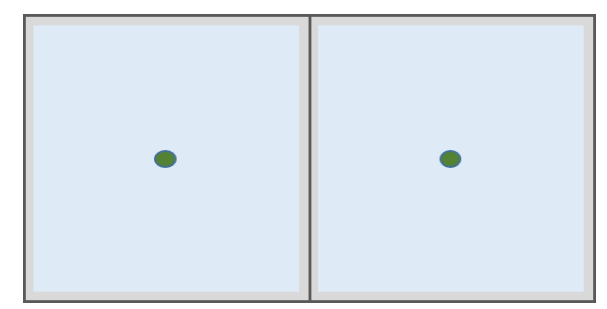

Figure 2.9: Cross-sectional view of a pair of outer detector scintillator scripts, which are embedded in a steel frame.

\subsubsection{Scintillator and Light Collection}

MINERvA uses polystyrene, a solid plastic scintillator with the chemical formula $(\mathrm{CH})_{8 n}$. It is extruded into triangular (rectangular) strips for the ID (OD). The scintillator is doped with $1 \%$ 2,5-diphenyloxazole (PPO) and 0.03\% 1,4-bis(5-phenyloxazol-2-yl) benzene (POPOP). Ionization caused by the passage of a charged particle induces PPO to scintillate. In response to the fluorescence of PPO, POPOP scintillates a more focused light spectrum at $410 \mathrm{~nm}$. Strips are coated with a white 15:85 mixture of $\mathrm{TiO}$ and polystyrene, which improves internal reflection.

A wavelength shifting (WLS) fiber is embedded in the center of each strip. WLS fiber collects the blue scintillation light, shifts it to green, and directs it out of the scintillator. The shifted green light is reflected internally in the fiber, which greatly reduces the loss of signal.

Groups of eight fibers are attached at a connector which plugs into a female end of an optical decoder unit (ODU) which protrudes from a steel tube, called a "box' 4 Boxes are mounted atop the detector. Eight connectors are plugged into each box 5 for a total of 64 fibers per box. The ODU is a set of 64 optical fibers which map light from the detector to the face of a 64-anode photomultiplier tube (PMT) to ensure that signals from adjacent strips do not go to adjacent PMT channels. Each box contains an ODU, PMT, and a PMT base, to which front end electronics will be attached. The digitization of this optical signal is discussed in the following

\footnotetext{
${ }^{4}$ Roughly half of the PMT boxes were assembled at Rutgers, The State University of New Jersey.

${ }^{5}$ Some boxes are not fully instrumented to the detector. These have a blackened plug inserted into one of the ODU ports to prevent light leaks.
} 
section.

\subsubsection{Electronics and Data Acquisition}

64-anode photomultiplier tubes (PMTs) transform the collected scintillation light into photoelectrons on their photocathodes, which are then amplified into a measurable signal by series of dynodes in the PMTs. There are $~ 500$ PMTs on the MINERvA detector which service $\sim 32,000$ channels $\sqrt{6}$ All of these PMTs were tested for quality assurance and calibration using the Rutgers PMT Test Stand. PMT signals are read by front-end boards (FEBs), which are controlled by a Field-Programmable Gate Array (FPGA). FEBs store charge for all 64 channels in six TriP-t chips and also power the PMT dynodes with a Crockcroft-Walton. Each TriP-t chip services 32 channels and has a user-selectable gain. Two chips are set to low gain for all 32 channels; the other four chips are set to medium- and high-gain for 16 channels each.

MINERvA reads out the entire detector for $16 \mu$ s at the call of a trigger signal from the NuMI beam. This readout window, hereafter referred to as a "gate", begins $0.5 \mu$ s before and ends $5.5 \mu$ s after the beam spill. When the charge in a channel crosses the discriminator threshold, the TriP-t's begin integrating charge and record hits times. All channels on a TriP-t are readout $150 \mathrm{~ns}$ after any channel fires the discriminator. There is a $20 \mathrm{~ns}$ reset window during which no charge is recorded in the recently readout channels. Channels can be readout in this way up to five times during each gate. In addition, there is an untimed final readout at the end of the gate, for which all hits are given the time of $16 \mu \mathrm{s}$, which is the end of the gate.

Groups of up to 10 FEBs are daisy-chained together and communicate with the data acquisition system (DAQ) through VME modules. Each chain of FEBs is connected to a Chain Readout Controller (CROC). Timing information, like the NuMI and MINOS triggers, is communicated among VME modules by a CROC Interface Module (CRIM). CROCs and a CRIM are mounted in a VME crate along with a CAEN V2718 crate controller, which communicates

\footnotetext{
${ }^{6}$ The number of instrumented channels varied with time.
} 
with a computer. MINERvA uses two VME crates.

For a more detailed description see Ref. [37].

\subsection{MINOS Near Detector}

The Main Injector Neutrino Oscillation Search (MINOS) is a two detector neutrino oscillation experiment situated on-axis in Fermilab's Main Injector [27]. The near detector is at Fermilab, and the far detector is $735 \mathrm{~km}$ away in Soudan, Minnesota. Both MINOS detectors are made of interleaved steel and plastic scintillator modules, similar to the MINERvA HCAL. Unlike MINERvA, the MINOS detectors are magnetized, which makes them capable of reconstructing the charge and momentum of the (anti)muons produced by charged current (anti)neutrino interactions.

The near detector, shown in Fig. 2.10, is $2 \mathrm{~m}$ downstream of MINERvA and composed of 282 planes, weighing in at 980 metric tons. Its transverse shape is a squashed octagon which resembles a witch's cauldron. MINERvA's optical system was based on that in MINOS, so there are many similarities. The major difference is that MINOS scintillator strips are rectangular with a cross sectional area of $1.0 \times 4.1 \mathrm{~cm}^{2}$. The steel modules are $2.65 \mathrm{~cm}$ thick. The near detector is divided longitudinally into two regions: the upstream calorimeter and downstream spectrometer. In the calorimeter, there is one scintillator plane between each pair of steel planes, which is adequate granularity for reconstructing the energy of a hadronic system. The spectrometer is meant to reconstruct muons and has one scintillator plane after every fourth steel plane.

Since the near detector is the only MINOS detector relevant to MINERvA, it is sometimes referred to in this dissertation as just "MINOS". For MINERvA's purposes, MINOS is a toroidal muon spectrometer. 

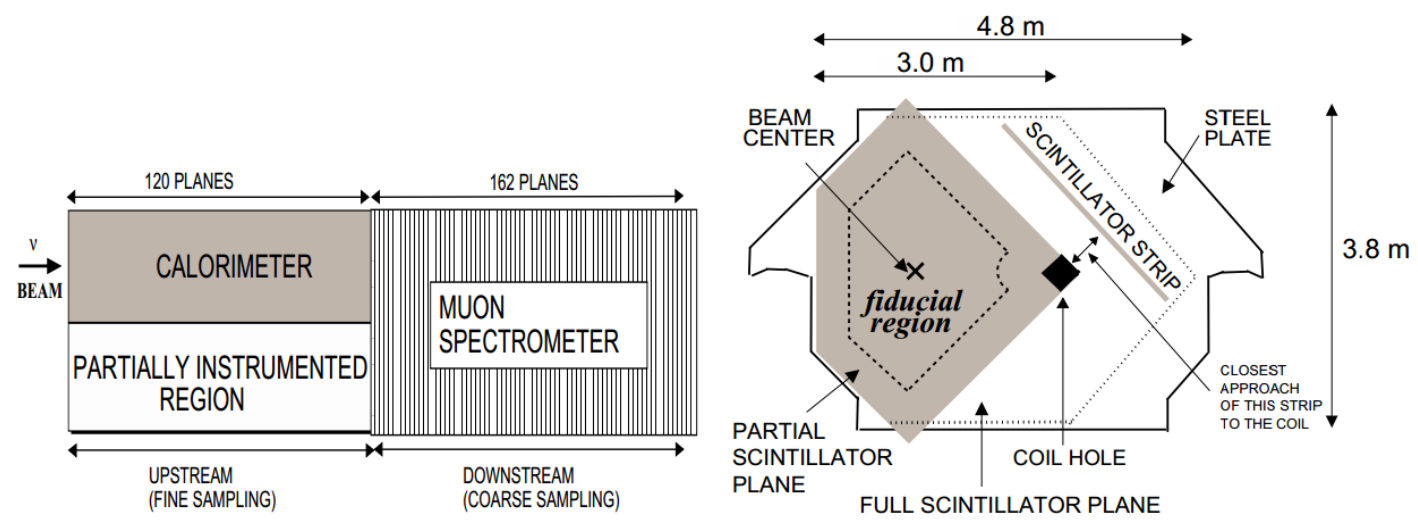

Figure 2.10: The MINOS near detector (left) as viewed from above and (right) as seen by the beam. Reprinted from [38] with permission from the MINOS collaboration; Copyright (2010) by The American Physical Society.

\subsection{Data Run Periods}

MINERvA has been collecting data since the fall of 2009. The Low Energy Run refers to data taken between the fall of 2009 and April 2012, shown in Ref. 2.11. This run ended when the accelerator complex was shut down for upgrades. The primary goal of the upgrades was to increase the energy, intensity, and repetition rate of the NuMI beam for NOvA. This is also quite beneficial for many analyses that can be performed in MINERvA. The upgrade was completed in September 2013, thus beginning the Medium Energy Run which will last at least another five years.

For its first four months of data taking, a partially-constructed ( $~ 50 \%)$ MINERvA recorded $\bar{\nu}_{\mu}$-mode data from NuMI. Neither this data nor the detector configuration are relevant to the analysis presented here. For information on and an analysis of data taken during this period see Ref. [39].

MINERvA was fully installed by the end of March 2010, with the exception of the water target, helium target, and veto wall. The detector recorded NuMI beam data in various beam configurations until the end of the Low Energy Run. Only the $\nu_{\mu}$-mode data is relevant to this dissertation. Most of the $\nu_{\mu}$ beam data was collected Summer 2010, and Fall 2010 through 


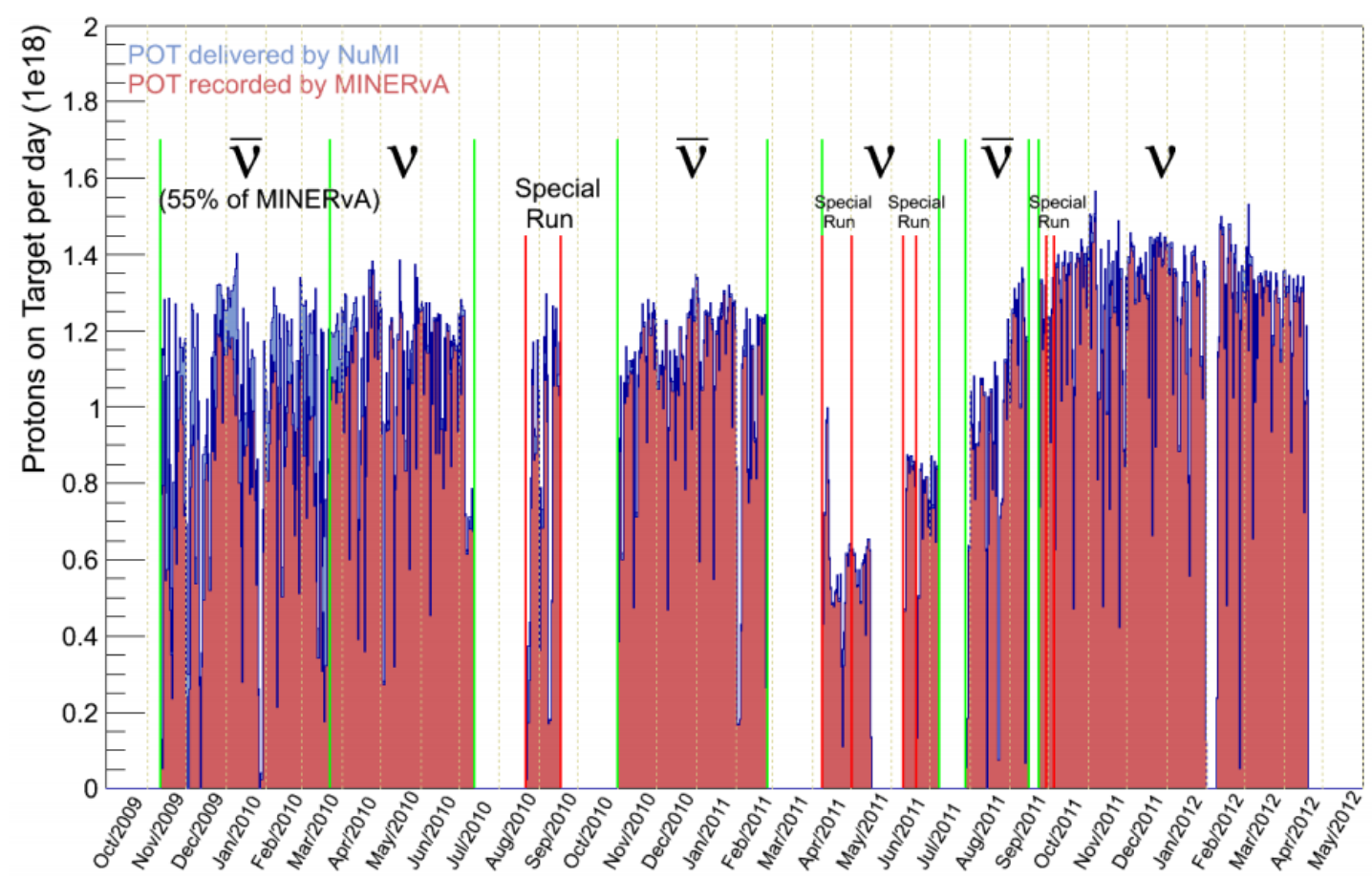

Figure 2.11: NuMI beam data recorded by MINERvA. The first $\sim$ four months of data were taken with $50 \%$ of the detector installed. Vertical bars indicate changes in the beam. Special runs refers to non-standard NuMI horn and target configurations.

Spring 2012. In addition, about one week of data was collected in each May and June of 2011.

Table 2.5 shows a summary of exposure to the NuMI beam in $\nu_{\mu}$-mode. 


\begin{tabular}{llll}
\hline Start & End & $\begin{array}{l}\text { P.O.T. } \\
\left(\times 10^{20}\right)\end{array}$ & Notes \\
\hline Mar 22, 2010 & Jul 12, 2010 & 0.941 & $\begin{array}{l}\text { 2 most upstream modules not instru- } \\
\text { mented. }\end{array}$ \\
May 7, 2011 & May 13, 2011 & 0.025 & \\
Jun 22, 2011 & Jul 1, 2011 & 0.064 & Veto installed. Helium and water \\
Oct 18, 2011 & Apr 30, 2012 & 1.914 & \begin{tabular}{l} 
targets present, sometimes empty. \\
\hline
\end{tabular} \\
\hline
\end{tabular}

Table 2.5: A summary of the data run periods during which MINERvA collected data from the $\nu_{\mu}$-focusing mode of the NuMI beam. P.O.T. listed is used for the analysis presented here and is less than the P.O.T. delivered to or recorded by MINERvA. 


\section{Chapter 3}

\section{Calibration and Reconstruction of MINERvA}

In the most basic terms, a particle detector measures the position, time, and energy of ionization caused by the passage of charged particles through its mass. These three quantities are not directly measured, but must be inferred from analog-to-digital conversion (ADC) and time-to-digital conversion (TDC) signals, and from knowledge of the detector's optical and electronic components. The detector must therefore be calibrated to account for any deviations or processes which are not modeled sufficiently well.

Reconstruction algorithms interpret calibrated measurements of energy deposits in the detector as particles. Pattern recognition and expectations for physical behavior in the detector form the basis of these algorithms. In neutrino scattering, all of the detector activity is due to the products of the interaction; neutrinos do not participate in electromagnetic processes like ionization. The process by which a neutrino interacted in the detector and the kinematics of that interaction are inferred based on the reconstruction of its final state products.

This chapter describes the calibration and reconstruction of raw hit information. It closely follows Ref. [26], although there are significant differences in the description of reconstruction.

\subsection{Hit Calibration}

Figure 3.1 shows the steps in collection of light and conversion to ADC that require calibration. Some calibrations were measured Ex situ on components prior to installation on the detector or with a separate bench test. Others are measured In situ using the fully constructed detector. The following is a summary of the effects that cause a priori measurements of detector 


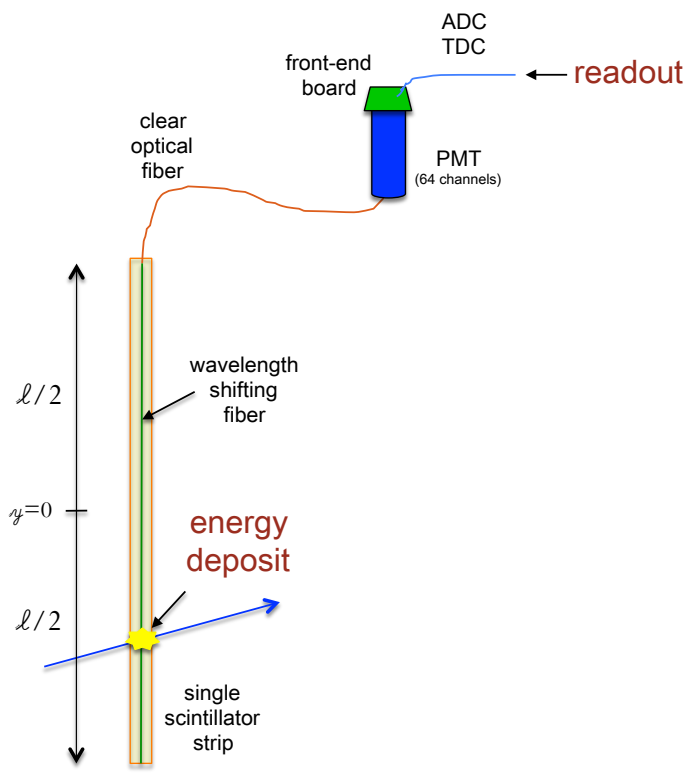

Figure 3.1: Schematic of effects accounted for in hit calibration. Figure taken from Ref. [26].

activity to differ from the actual activity and thus must be calibrated.

\section{Position}

The most dramatic difference between the true and observed position happens when a channel is not connected to the strip predicted by the plex, which is the mapping of electronics to detector channels. There are deviations from the nominal position of modules due to imperfections in construction, and the stress and shear from adjacent modules.

\section{Time}

Calibrations must account for the time it takes light to travel though the optical fibers to reach the PMTs. This calibration is different for all channels because of variations in fiber lengths and imperfections of fibers. The fluorescence time of the PPO/POPOP system is energy-dependent. The time it takes FEBs to respond varies due to their different distances from the VME crate and also inherent variations in manufacturing. 


\section{Energy}

Many corrections must be made to interpret ADC counts as energy depositions in the detector. The conversion of measured $A D C_{i}$ to energy deposition $E_{i}$ in channel $i$ can be parameterized as

$$
E_{i}=A D C_{i} \times\left[Q_{i}(A D C) \cdot G_{i}(t) \cdot e^{l_{i} / \lambda_{\text {clear }}} \cdot \eta_{i}^{\text {att }}(d) \cdot S_{i}(t) \cdot C(t)\right]
$$

where the calibration terms are:

- $Q_{i}(A D C)-$ Conversion of charge to ADC, or gain, of the TriP-t chip.

- $G_{i}(t)$ - Amplification of signal in the PMT dynode chain.

- $e^{l_{i} / \lambda_{\text {clear }}}-$ Attenuation of light in the clear optical fiber with attenuation length $\lambda_{\text {clear }}=$ $7.83 \mathrm{~m}$.

- $\eta_{i}^{a t t}$ - Attenuation of light in the WLS fiber, which is a function of the position in the fiber.

- $S_{i}(t)$ - Time-dependent relative energy scale of the channel with respect to the others. This accounts for variations in the extrusion of scintillator, bubbles in optical fibers, connection of the fibers to the PMT, environmental conditions across the detector (temperature, humidity, dripping water), and any other variations.

- $C(t)$ - Time-dependent absolute energy scale.

\subsubsection{Ex situ Calibrations}

Some calibrations were measured on components prior to installation on the detector or with a separate bench test. 


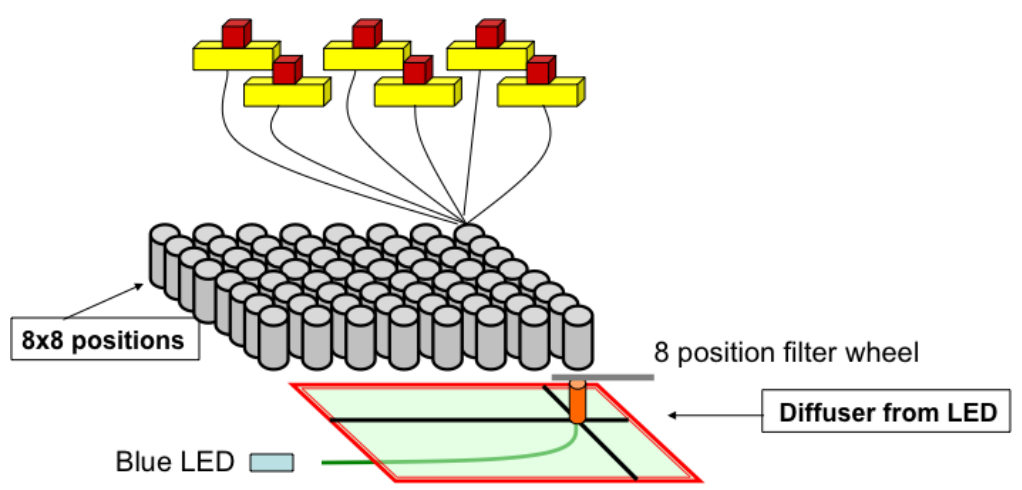

Figure 3.2: Schematic of the Rutgers PMT test stand. Light from a blue LED is directed though a green WLS fiber and illuminates one pixel in each of six PMTs. A diffuser can filter light to vary the signal. Figure taken from Ref [26].

\section{Front-End Boards}

The conversion of charge to ADC count was measured on each FEB for all three gain settings (low, medium, high) prior to installation. A known charge from a capacitor was injected into the FEB, and the resulting ADC count response was fit to a tri-linear function to calibrate each channel.

\section{Photomutliplier Tubes}

PMTs were tested on a dedicated test stand at Rutger ${ }^{1}$ before being enclosed in steel boxes. The test stand illuminated one pixel at a time using light from a blue LED that was directed through a green WLS fiber. 64 groups of six clear optical fiber were used so that six PMTs could be tested at once. Figure 3.2 shows the setup.

\section{Module Mapper}

The optical attenuation in the WLS fibers in each scintillator plane was mapped using $\gamma$ radiation of a ${ }^{137} \mathrm{Cs}$ source. The source was directed up and down each strip in the plane longitudinally while strip response was measured. The resulting calibration is an attenuation

\footnotetext{
${ }^{1}$ The Rutgers test stand was moved to Fermilab in May 2010 to test replacement PMTs. The test stand is still able to test PMTs should the need arise during the Medium Energy Run.
} 
scale factor for each channel that is a function of the energy deposition's longitudinal position in the strip.

\subsubsection{In situ Calibrations}

In situ calibrations are necessary to characterize time-dependent effects, and account for variations in or changes due to detector construction. Measurements for these calibrations use either rock muons or special triggers, which are explained below.

Rock muons are the final state products of neutrino interactions in the rock of the detector hall that enter MINERvA. They are plentiful; often, several are recorded in a beam spill. Muons are minimum ionizing particles, thus their energy deposition pattern in the detector is consistent and well understood.

The MINERvA DAQ can send triggers to readout the detector between NuMI spills, provided there is enough time to be ready to trigger for the next spill. There are two special types of trigger important for calibration: pedestal and light injection. The specifics of each are discussed below.

\section{Pedestals}

Reading an FEB will result in a nonzero ADC count even when no charge has been recorded. This ADC count is called the "pedestal". The pedestal is measured simply by reading out the detector when there is expected to be no light. This is accomplished by taking pedestal triggers between NuMI triggers, when there is no beam. The mean of the ADC distribution is taken as the value of the pedestal and subtracted during calibration. Each channel has its own pedestal calibration. Pedestal data is taken by switching to a mixed NuMI-pedestal trigger mode roughly twice a day for one half hour each. 


\section{PMT Gains}

The gain of each PMT channel changes as it ages. The ideal way to measure gain is to induce a known number of photoelectrons to cascade through the dynode chain and measure the response. Such a system would have been very expensive and not entirely necessary for MINERvA. Instead, MINERvA employs a light injection system. Each PMT box has two ports into which optical fibers from a light injection system are connected. There is a diffuser in each port to spread the light across the whole face of the PMT. The LED used in light injection is tuned so that roughly one photoelectron is generated in each channel. Assuming a probabilistic model for amplification in the dynode chain, the gain can be calculated from the difference in the RMS of the pedestal ADC and of the one-photoelectron ADC. The gain is calculated for all channels in the detector. Light injection data is taken between NuMI spills, in a mixed trigger mode.

\section{Plane Alignment}

Installation and gravity cause small shifts in position and rotation of scintillator planes. These deviations can be measured by comparing the fitted path of a rock muon though a strip and the energy deposited in that strip. The farther a muon travels through a strip, the more energy it will deposit in said strip. Since most rock muons are along the beam direction, the longest distance traveled is through the point of the scintillator triangle. The energy deposited in a strip is histogrammed as a function of transverse position. Finding the peak energy deposition from the histogram locates the point of the triangle. This is done for all strips in the plane. A shift in position will cause the peak to be found offset from nominal position. A rotation about the $\mathrm{z}$ axis manifests as a positional dependence through the plane of the shift in the triangle peak position. Figure 3.3 shows an example of the measurements used for alignment. 

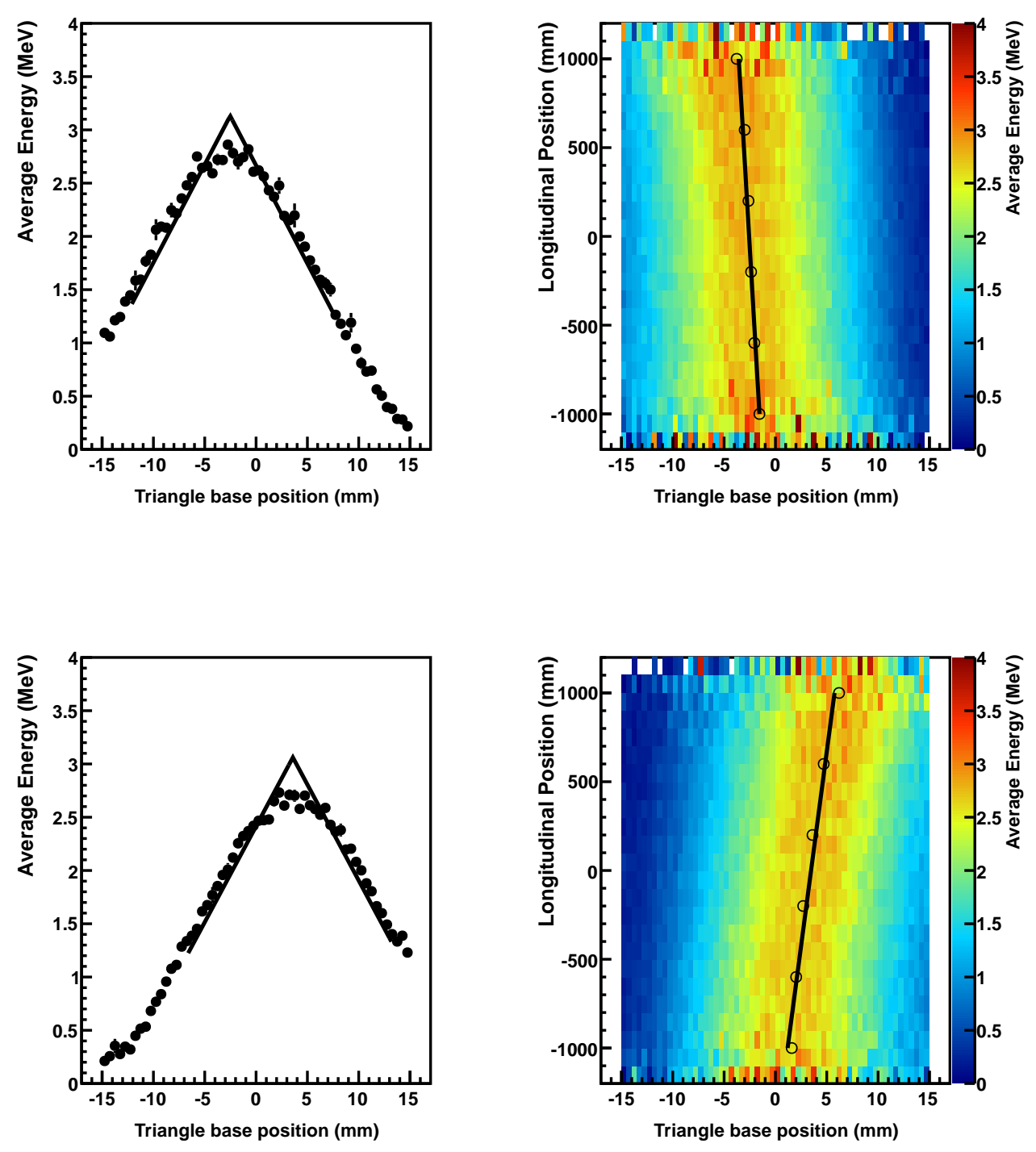

Figure 3.3: Alignment of a scintillator plane using the measured position and $d E / d x$ with a rock muon sample. (left) The peak of the energy distribution is offset from the expected value of 0 meaning there is an offset in strip position. (right) The triangle base position changes with the longitudinal position in the strip, indicating a rotational misalignment. The top and bottom rows are different planes. Figure from Ref. [26]. 


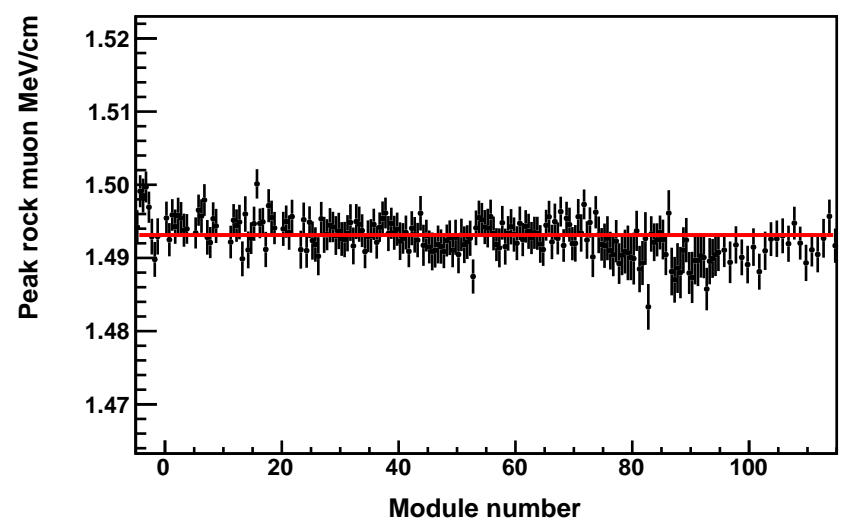

Figure 3.4: Peak muon energy per length for for all planes. The consistency in energy response is due to the channel-to-channel calibration. The red line is a linear fit showing flatness. Figure from Ref. [26].

\section{Channel to Channel Calibrations}

There are numerous reasons for remaining variations in the channels' responses to energy deposition. A channel-to-channel calibration is performed to normalize channels relative to each other, thereby removing any remaining variations. The calibration is performed on a sample of tracked rock muons that enter the front and exit the back of MINERvA; such muons are called through-going muons. The energy per length, $E / L$, of scintillator traversed is measured, as in the process of plane alignment. It is important that the plane alignment calibration is applied first. Variations in the peak of the $E / L$ distribution are calibrated out. Consistency in the energy response of modules after channel-to-channel calibration is shown in Fig. 3.4

\section{Absolute Energy Scale}

The absolute energy scale of the detector is set by comparing the peak energy deposited by a muon in a scintillator strip, called the muon energy unit (MEU), in data to simulation. The use of simulation is safe because the mechanism of muon energy loss through ionization is very well understood. Through-going rock muons with a reconstructed momentum are used 

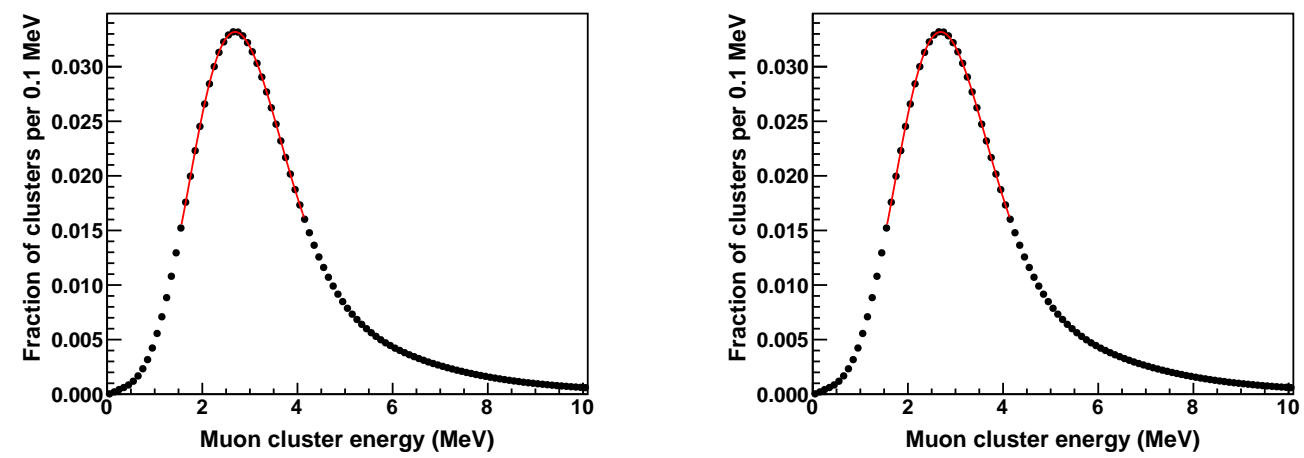

Figure 3.5: Calibration of absolute energy scale using muon energy unit (MEU). (Left) Comparison of MEU in data and simulation used to set the calibration. (Right) Fit to the peak of the energy distribution gives the value for MEU. Figure from Ref. [26].

for the calibration. The MEU is extracted from a fit of the distribution of energy deposited by the muons. The calibration is the ratio of data to simulation for this value. Only one scale factor is necessary for the detector since the previous channel-to-channel calibration removes any variations. Figure 3.5 shows a a comparison of data and simulated muon energy deposited per length and a fit to the distribution to tune the MEU.

\section{Timing}

Timing calibrations are also accomplished using fitted through-going rock muon tracks. The calibration procedure is iterative; the best known timing calibrations are applied to find even better timing calibrations until convergence. The time of each hit is compared with the truncated mean time of hits on the track to form a residual, taking into account the travel time of the muon and previous timing calibrations.

Time slewing is calibrated for each channel. The calibration moves the center the peak of the hit time residual to 0 . The slewing calibration is done in bins of energy deposited, because it is energy-dependent.

A separate time calibration accounts for the time offset between FEBs. This calibration is 
done for each group of 32 channels connected by a TriP-t chip.

\section{Cross-talk}

Cross-talk is measured in situ using rock muon tracks. The ideal measurement of crosstalk illuminates just one pixel at a time, as in Sec. 3.1.1. Muons are an adequate light source because they usually deposit energy into only two strips per plane. Cross-talk is identified as signal measured in channels that were not traversed by the muon but sit on the same PMT as channels that were traversed by the muon. The frequency and energy distribution of crosstalk hits is compared to the simulation to calibrate the probabilistic algorithm that simulates cross-talk.

\subsection{Reconstruction}

This section describes a track-based approach to finding events with a muon in MINERvA. Such events can be charged current neutrino interactions or rock muons.

Reconstruction of events in MINERvA begins by grouping hits in a gate temporally into "time slices", which usually are collections of all the activity produced by physical events in the detector. Subsequent reconstruction algorithms act on single time slices, and hits in different time slices do not interfere ${ }^{2}$ Hits are then spatially grouped within planes into "clusters". Groups of clusters that resemble the path of a charged particle are associated as a "track". Tracks are fit to the expected path of a charged particle to better understand its path. Tracks are identified as muons by looking for corresponding activity in MINOS. Clusters that are not associated with the muon are assumed to come from the recoil system, and their energies are summed to get the recoil energy.

\footnotetext{
2 This is not true for the reconstruction of Michel electrons. Hits from Michels are seen roughly $2 \mu \mathrm{s}$ after the hits from the parent $\mu^{+}$or $\pi^{+}$due to the delay in decay. The time difference is large compared to the typical separation of time slices, so the Michel will be found in a later time slice. Michel electrons are not considered in this analysis.
} 


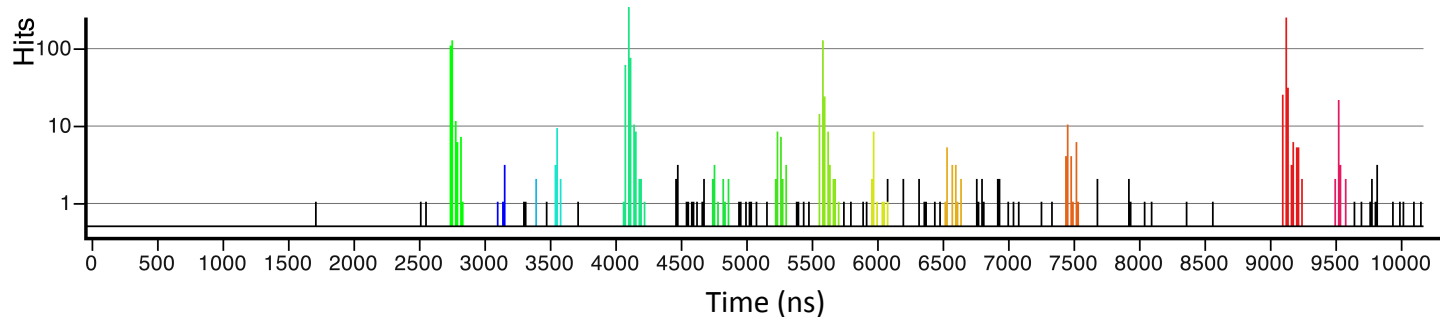

Figure 3.6: Hit time distribution in a neutrino beam spill. The colored peaks are hits that have been grouped together into "time slices". Figure comes from Ref. [26].

\subsubsection{Clustering Hits by Time}

Time slices are found by scanning the hit time distribution. The algorithm starts by walking a $30 \mathrm{~ns}$ window forward in time. If the integrated number of photoelectrons in the window exceeds 10, a candidate time slice is created. All hits in the $30 \mathrm{~ns}$ window are part of the time slice. The window then continues to talk forward in time, adding all hits in the roaming window to the time slice, until the integrated number of photoelectrons is less than 10 . The window then resumes walking forward and will look for more time slices.

Figure 3.6 shows an example hit time histogram for a gate. Colored peaks in the distribution are time slices. A typical beam spill from $\mathrm{NuMI}$ in $\nu_{\mu}$-focusing mode contains 5-12 time slices.

\subsubsection{Clustering Hits by Position}

The first step of spatial pattern recognition is merging hits within a plane into clusters. Hits that are contiguous are merged into a cluster. Clusters take hits in a greedy fashion, i.e. a cluster is made out of the maximum number of contiguous hits. All hits are used to create clusters, which necessitates the existence of one-hit clusters.

The energy of a cluster is the sum of energies of its constituent hits. Its time is taken from the time of the most energetic hit. The cluster position is the charge-weighted mean position of the hits.

Because of the alternating triangular design in scintillator planes, it is extremely likely that 
a particle passing through a plane will intersect more than one strip. A different topology of hits is expected for different situations. For example, muons tend to produce very narrow clusters, and showering hadrons produce very high energy broad clusters. Clusters are classified as low activity, trackable, heavy ionizing, cross-talk, or supercluster according to their energy, number of hits, and energy of hits. Hits are classified by their energy. The classification criteria for clusters is as follows.

- Low activity clusters have energies less than $1 \mathrm{MeV}$.

- Trackable clusters have energies between 1-12 MeV and contain fewer than five hits. The cluster must have at least one hit with energy $0.5-12 \mathrm{MeV}$, but no more than two. If there are two hits with $0.5-12 \mathrm{MeV}$, they must be adjacent.

- Heavy ionizing clusters have energies greater than $12 \mathrm{MeV}$ and contain fewer than five hits. The cluster must have at least one hit with energy greater than $0.5 \mathrm{MeV}$, but no more than three. If there are two or three hits with energies greater than $0.5 \mathrm{MeV}$, they must be contiguous.

- Cross-talk clusters are made of hits that are correlated with tracked activity in PMT space.

- Superclusters do not fit the criteria of any of the above categories. Practically, this means clusters that are very wide or have hit patterns consistent with multiple particles are superclusters.

\subsubsection{Track-Based Event Formation}

The analysis described here looks for charged current events, i.e. events with a muon in the final state. Muons are the simplest final state particle to identify and, in this case, the most important. For these reasons, MINERvA has developed an algorithm to build events which is focused primarily on finding the muon track. 


\section{Identify the Anchor}

The first step in the event formation algorithm is to find tracks by applying pattern recognition to trackable and heavy ionizing clusters. The longest track, usually the muon, is declared the "anchor" track and retained. All non-anchor tracks are discarded. Anchor tracks must be longer than 25 nodes, or the event is discarded. The event vertex is taken to be the origin of the anchor track.

\section{Create Anchored Tracks}

Next, pattern recognition is redone with the clusters that were not used to create the anchor. If tracks are found to be compatible with the event vertex, they are kept. The criteria for compatibility are (1) that the track's projection is less than $100 \mathrm{~mm}$ away from the vertex at its closest approach and (2) that the track's origin is less than $250 \mathrm{~mm}$ away from the vertex. If any anchored tracks are found, this step is repeated. Each iteration of pattern recognition uses fewer clusters, because the clusters have been used to make tracks.

\section{Create Secondary Tracks}

Next, secondary tracks are created by finding tracks that begin near the end of a primary track. A track emanating from terminus of another track can be the result of an inelastic scatter, decay, or hard elastic scatter. The anchoring test is the same for secondary tracks as explained above, except that the anchor point is the end of a track instead of the primary vertex. This step is repeated until no more new tracks are found.

Repeating each step until convergence ensures that all tracks which can be traced back to the primary vertex through a connected system of tracks are found. Tracks and vertices are fit after each pattern recognition step as described in Sec.3.2.5. 


\subsubsection{Track Formation}

Track formation has two steps: track finding and track cleaning.

\section{Track Finding}

MINERvA employs a track seed-based pattern recognition scheme to find tracks. Here, a track is a group of clusters that have a pattern consistent with the path of a charged particle. Clusters in a time slice that have not yet been associated with a track are the inputs to the track finding algorithm.

First, track seeds are created by comparing the positions of clusters that are in the same view $(\mathrm{X}, \mathrm{U}$, or $\mathrm{V})$. Track seeds are made of three clusters that are in the contiguous planes of a particular view and can be fit to a $2 \mathrm{D}$ line with reasonable success.

Next, track candidates are grown by stitching together compatible track seeds to make longer 2D lines. To form a track candidate, the seeds must be in the same view, share one cluster, and have similar slopes. After candidates are formed, they are joined. Track candidates are joined with other candidates in the same view if they project to each other. Compatible candidates are joined even if there are gaps instead of clusters between them.

The last step is the merge candidates of different views. There are two schemes for doing this: 3D and 2D. In 3D merging, triplets of track candidates are tested for longitudinal overlap and 3D consistency, i.e. that the coordinates calculated with two candidates correctly predict the third. In 2D merging, pairs of track candidates are tested for longitudinal overlap and 3D consistency. The 3D consistency for 2D merging requires untracked activity where one would expect a third candidate, rather than requiring an actual third track candidate. 3D merging is more robust and is tried to exhaustion before $2 \mathrm{D}$ merging is allowed to run. $2 \mathrm{D}$ merging is useful because it finds tracks which are obscured in one view by overlapping activity or detector inefficiency. 


\section{Track Cleaning}

During track cleaning, clusters may be added to a track or partially broken apart. Clusters can be added to a track for two purposes:

- Fill gaps in tracks that cross planes in which there is no activity associated with the track. If there are clusters in the gaps, they are added to fill the gap.

- Extend endpoints of the track by attempting to propagate farther upstream. Sometimes the upstream end of a muon's track is obscured by hadronic activity. In this cleaning step, the track will claim muon-like amounts of energy from clusters that are in its upstream path of propagation.

Sometimes a cluster is added to a track even though it was already used by another track. These situations are resolved by breaking the cluster in two. The fraction of the cluster energy awarded to each track is determined by the $d E / d x$ of each track near the disputed cluster. The most common case is a muon that possesses added superclusters or heavy ionizing clusters. In this instance, the muon track gets an amount of the shared cluster's energy that is consistent with the muon's $d E / d x$ in the vicinity of the disputed cluster.

\subsubsection{Track and Vertex Fitting}

To fit tracks, MINERvA uses an implementation of the well-known Kalman filter method [40], which is a local least-squares estimator. During the application to track fitting, the Kalman filter will minimize the sum of standardized distance between the position of the energy deposited in a layer of scintillator and the estimate of the track's parameters as it passed through that layer of scintillator. The algorithm produces a position, slope, and covariance matrix for each cluster. The covariance matrix includes noise from multiple scattering [41].

Vertices with at least two tracks are initially fit with a crude (though often very good) estimate of the vertex position by averaging points of closest approach. The vertex position is 
then fit using an adaptive Kalman filter minimization routine [42]. The adaptive feature weights down tracks that do not appear to be compatible with the vertex, so that they influence the vertex position less.

For more details on fitting methods see Appendix C.

\subsubsection{Muon Reconstruction}

The proper identification and reconstruction of muons is crucial to the analysis presented in this dissertation. A track in MINERvA is identified as a muon if it matches to activity in MINOS. These are the only types of muons currently used in charged current inclusive analyses.

\section{Matching Tracks to MINOS}

MINERvA tracks that have at least one cluster in any of the five most downstream modules (in the HCAL) are considered candidates for matching. MINOS track-match candidates must have hits in at least one of the four most upstream MINOS planes. The MINOS and MINERvA tracks must occur within 200 ns of each other. All pairs of candidates are tested for compatibility in two ways.

The first, and preferable, compatibility test projects the tracks to each other's end points. The position and slope of the MINERvA track at its most downstream point is projected downstream into MINOS and compared with the MINOS track-match candidate's most upstream position. The MINOS track is propagated upstream to MINERvA for a crosscheck. If the residual of both projections is less than $40 \mathrm{~cm}$, the two tracks are matched. If more than one MINOS track passes the matching criteria, the one with the lowest projection residual is used.

The second test uses the distance of closed approach, and is used only if no track-match is found using the endpoint projection criteria. The MINERvA and MINOS tracks are simultaneously projected towards each other. The projection compatibility test is then evaluated at the 
point of closest approach. This matching method exists to find cases in which the muon scatters off material between MINERvA and MINOS.

\section{Charge and Momentum Reconstruction}

The magnetic field in MINOS deflects muons according to their charge. The charge of the muon determines the sign of curvature of this deflection. Deflection of the muon is measured, and the charge is inferred. The polarity of the field can be flipped so that either helicity can be focused: usually $\mu^{-}$for $\nu_{\mu}$ studies and $\mu^{+}$for $\bar{\nu}_{\mu}$ studies.

The momentum of a muon is reconstructed starting at the downstream end, with MINOS. MINOS uses two methods of momentum reconstruction: range and curvature. Reconstruction by range is the more precise method but is only possible for lower energy, lower angle muons that stop in the detector. Muons are reconstructed by curvature described by

$$
K=\frac{1}{R}=\frac{0.3 B}{p_{\mu}}
$$

The energy which the muon loses in its travel through MINERvA is then added.

\subsubsection{Recoil System Reconstruction}

Clusters in the recoil system pass the following criteria:

- They are not associated with the muon track.

- They are not identified as cross talk.

- They are within a time window of $-20 /+35$ ns of the event time.

The energy of these clusters is added calorimetrically to determine the energy of the recoil system. Three corrections are applied to the clusters' energy:

1. Correct visible energy by accounting for passive material traversed. 
2. Multiply the above result by a scale factor $s_{i}\left(E_{\text {recoil }}\right)$ that is tuned to Monte Carlo.

3. Apply a polyline correction to the above result that applies an energy-dependent scale factor, also tuned to Monte Carlo.

The reconstruction procedure can be summarized as

$$
E_{\text {had }}=\operatorname{poly}\left(E_{\text {had }}\right) \cdot \alpha \cdot \sum_{i}^{\text {hits }} E_{i}^{v i s} \cdot f_{i}^{\text {pass. }}
$$

where $\operatorname{poly}\left(E_{h a d}\right)$ is the polyline correction, $\alpha$ is constant scale factor that depends on the vertex position, $E_{i}^{v i s}$ is the visible energy of a hit, and $f_{i}^{\text {pass. }}$ corrects for expected loss of energy in passive material near the hit.

\section{Passive Material Correction}

The passive material traversed by the particle is estimated using the hit location. To calculate how much energy is lost in the passive material, it is assumed that the number of MEUs per $\mathrm{g} / \mathrm{cm}^{2}$ deposited in scintillator is also deposited in the passive material. The number of MEUs deposited in scintillator is

$$
n_{\text {meu }}=\frac{e_{\text {vis }}}{f_{\text {active }} \cdot M_{S c .} \cdot d E d x_{S c}},
$$

where $e_{v i s}$ is the visible energy of a hit, $M_{S c}$. is the mass of scintillator that recorded the hit in $\mathrm{g} / \mathrm{cm}^{2}$, and $d E d x_{S c}$ is the energy lost in scintillator by a minimum ionizing particle in $\mathrm{MeV} /$ $\mathrm{g} / \mathrm{cm}^{2}$. The total energy deposited is estimated to be

$$
E=n_{\text {meu }} \cdot \sum_{i}^{\text {materials }}\left(M_{i} \cdot d E d x_{i}\right),
$$

where the sum is over materials near the hit. The $d E / d x$ values are listed in Table 3.1 .

When a hit is in a plane adjacent to a passive nuclear target it is assumed that half of the target's thickness has been traversed. Hits provide only 1D information about the transverse position and most hits in the recoil system are not tracked. Therefore it is not generally possible 


\begin{tabular}{c|cccc}
\hline Material & Scintillator & Carbon & Iron & Lead \\
$\mathrm{dEdx}\left(\mathrm{MeV} / \mathrm{g} / \mathrm{cm}^{2}\right)$ & 1.936 & 1.742 & 1.451 & 1.122 \\
\hline
\end{tabular}

Table 3.1: $d E / d x$ for materials in the MINERvA detector [43].

\begin{tabular}{c|ccccc}
\hline Vertex Location & Target 2 & Target 3 & Target 4 & Target 5 & Tracker \\
\hline$\alpha$ & 1.78 & 1.67 & 1.59 & 1.57 & 1.60 \\
\hline
\end{tabular}

Table 3.2: Calorimetric scale factors $\alpha$ from Eq. 3.3 .

to associate a hit with carbon, iron, or lead in the passive target. Instead, the average $d E / d x$ of target materials is used which takes into account the area of the target occupied by each material along the length of the hit strip. For details on the calculation of strip lengths associated with passive materials, see Appendix B.

\section{Multiplicative Scale Factor}

The multiplicative scale factor $\alpha$ is applied to the reconstructed recoil energy to account for additional losses of visible energy like finite containment, neutral particles, and FSI. The scale factor is tuned using the Monte Carlo by minimizing the error between the reconstructed recoil energy and the true hadronic energy, defined as $E_{h a d}=E_{\nu}-E_{\mu}$. This definition of true recoil energy is chosen to optimize the neutrino energy reconstruction, which is reconstructed for inclusive events as $E_{\nu}=E_{h a d}+E_{\mu}$. Only events that pass all analysis cuts and have true $E_{\text {had }}$ between 1 and $10 \mathrm{GeV}$ are used for the tuning. A different $\alpha$ is found for the tracking region and for each passive target, because showers emanating from different locations in the detector encounter different material and thus develop differently. Scale factors are shown in Table 3.2 . 


\section{Polyline Correction}

An additional multiplicative correction is derived from the residuals in bins of $E_{\text {true }}$. A polyline is formed with points $(X, Y)=\left(\bar{E}_{\text {true }}(1+\mu), \bar{E}_{\text {true }}\right)$, where $\bar{E}_{\text {true }}$ is the average true $E_{h a d}$ in that bin and $\mu$ is the mean of the Gaussian fit for the residual $\left(E_{\text {reco }}-\right.$ $\left.E_{\text {true }}\right) / E_{\text {true }}$ in that bin. The polyline starts at $(0,0) \mathrm{GeV}$ and ends at $(50,50) \mathrm{GeV}$. Bins with $E_{\text {true }}<300 \mathrm{MeV}$ are not used. The multiplicative correction for an event with $E_{\text {had }}$ is determined from the two polyline points $p^{1}$ and $p^{2}$ such that $p_{X}^{1}<=E_{h a d}<p_{X}^{2}$. The energy after the polyline correction is

$$
E_{\text {had }}^{\prime}=p_{Y}^{1}+\left(E_{h a d}-p_{X}^{1}\right) \cdot \frac{p_{Y}^{2}-p_{Y}^{1}}{p_{X}^{2}-p_{X}^{1}}
$$




\section{Chapter 4}

\section{Simulation}

The simulation of neutrino events in MINERvA is accomplished in four distinct steps. Each of these steps employs at least one Monte Carlo 1 . First, G4numi predicts the neutrino flux produced by NuMI. Next, GENIE determines if and how these neutrinos interact in the MINERvA detector. Then, Geant4 and MINERvA-specific codes simulate the detector response to the final state products of the neutrino interaction. Last, any simulated particles exiting the back of MINERvA are propagated to and simulated in MINOS with Geant3.

\subsection{NuMI Flux}

The NuMI beam is simulated with the G4numi package, which is a NuMI-specific implementation of Geant4 version 9.2.p03. MINERvA has chosen to use the FTFP_BERT hadronic physics model for G4numi. For energies greater than $5 \mathrm{GeV}$, FTFP_BERT uses the FRITIOF string model to generate the primary hadronic collision, the Lund model for fragmentation into hadrons, and precompound splines to de-excite the remnant nucleus. The Bertini model for intranuclear cascade is used for lower energy hadrons.

G4numi has a description of the NuMI beamline geometry. $120 \mathrm{GeV} / c p \mathrm{C}$ collisions are generated one proton at a time with a beam spot size of $1.1 \mathrm{~mm}$. The hadronic models used in the simulation were found to disagree significantly, so external hadron production data is used to reweight these predictions. This reweighting is significant, but this is discussed in Sec. 4.1 .

\footnotetext{
${ }^{1}$ All simulations described in this dissertation are Monte Carlo codes. "Simulation" and "Monte Carlo" may be used interchangeably.
} 
The products of primary $p \mathbf{C}$ collisions are allowed to propagate through the material of the NuMI beamline, where they may reinteract. The products of these interactions are focused according to a description of the magnetic horns and decayed by G4numi.

This procedure is used to predict the flux from NuMI for all neutrino and antineutrino flavors.

\section{Reweighting pC Interactions}

As mentioned above, the FTFP_BERT model does not provide a very precise description of proton-nucleus interactions. To improve the accuracy of the flux prediction, MINERvA reweights events according to measurements from $p C$ hadron production experiments. Currently, results from two hadron production experiments are used for reweighting:

- NA49 [44] - Collected data at $158 \mathrm{GeV} / c$. NA49 also has the more descriptive handle "Large Acceptance Hadron Detector for an Investigation of Pb-induced Reactions at the CERN SPS".

- Barton et al. [45] — Collected data at $100 \mathrm{GeV} / c$ using the Fermilab Single Arm Spectrometer in the M6E beamline.

Neither of these datasets was collected at the NuMI momentum $120 \mathrm{GeV} / c$, so an energy scaling is applied using predictions from FLUKA [46], a different hadron production simulation.

Figure 4.1 shows a comparison of the $p \mathrm{C} \rightarrow \pi^{ \pm} X$ cross sections predicted by the FTFP_BERT model to data collected with NA49 [44]. The kinematic region relevant to $\nu_{\mu}$ production for MINERvA is approximately $p_{t}=[0.1,0.6] \mathrm{GeV} / c$ and $x_{F}=[0.05,0.15]$.

The reweight factor is the ratio of data to FTFP_BERT values of the invariant cross section

$f\left(E, x_{F}, p_{t}\right)=E \frac{d^{3} \sigma}{d p^{3}}$. An energy scaling correction calculated with FLUKA accounts energy difference between NuMI energies and available data. $p_{t}$ is the outgoing particle's transverse 


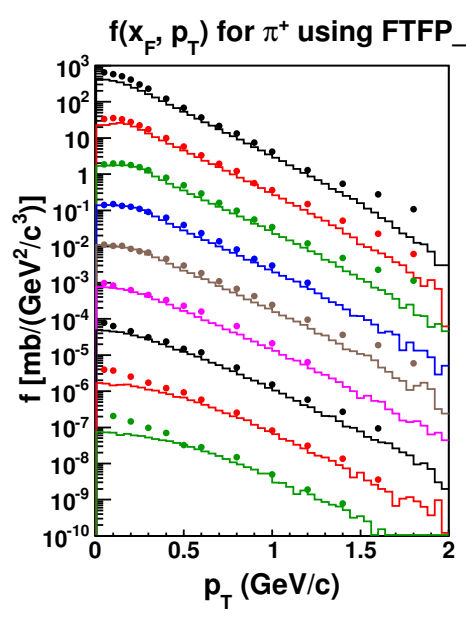

(a) $p C \rightarrow \pi^{+} X$

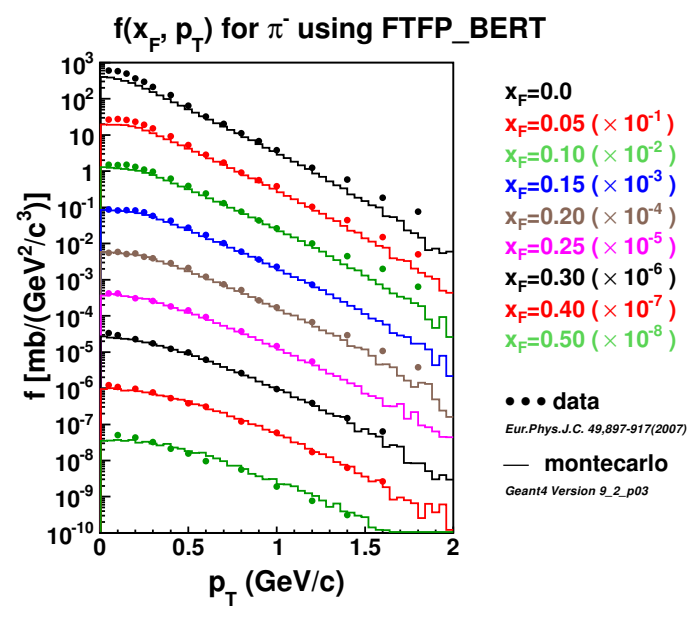

(b) $p C \rightarrow \pi^{-} X$

Figure 4.1: A comparison of NA49 and FTFP_BERT predicted hadron cross sections for $p$ C $\rightarrow$ $\pi^{+} \mathrm{X}$ (left) and $p \mathbf{C} \rightarrow \pi^{-} \mathrm{X}$ (right).

\begin{tabular}{ccc}
\hline Cross Section & Kinematics & Dataset \\
\hline$p C \rightarrow \pi^{ \pm} X$ & $x_{F}<0.5$ & NA49 [44] \\
$p C \rightarrow \pi^{ \pm} X$ & $x_{F}>0.5$ & Barton et al. [45] \\
$p C \rightarrow K^{ \pm} X$ & $x_{F}<0.2$ & NA49 [44] \\
$p C \rightarrow p X$ & $x_{F}<0.95$ & Barton et al. [45] \\
\hline
\end{tabular}

Table 4.1: Summary of hadron production interactions constrained by measurements.

momentum and a scaling variable from Feynman $x_{F}=2 p_{L}^{*} / \sqrt{s}$, where $p_{L}$ is outgoing particle longitudinal momentum and $\sqrt{s}$ is the total center of mass energy [47]. The factor for reweighting a simulated event with $x_{F}$ and $p_{t}$ to data collected at $158 \mathrm{GeV} / c \approx 158 \mathrm{GeV}$ is then

$$
R W=\frac{f\left(158 \mathrm{GeV}, x_{F}, p_{t}\right)_{\text {data }}}{f\left(E, x_{F}, p_{t}\right)_{g 4 n u m i}} \cdot \frac{f\left(E, x_{F}, p_{t}\right)_{\text {fluka }}}{f\left(158 \mathrm{GeV}, x_{F}, p_{t}\right)_{\text {fluka }}} .
$$

Particles that reinteract are subject to similar reweighting. Weights are also applied to account for the attenuation of the proton beam in the NuMI target.

Some simulated events are outside of the kinematic range of the data or produce a final state that was not measured. These events are not constrained and do not get a reweight factor $(R W=1)$. The kinematic regimes that are reweighted are summarized in Table 4.1 


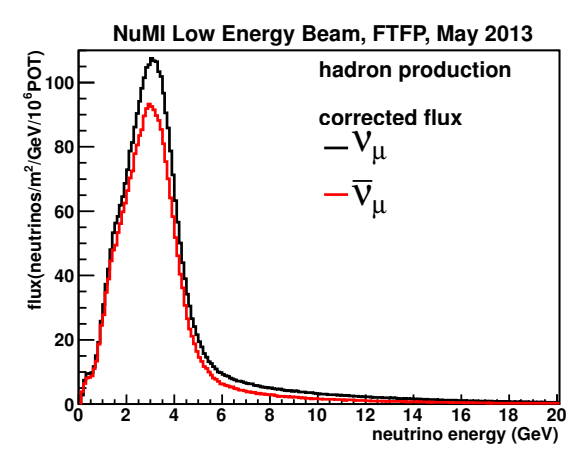

(a) Predicted Flux

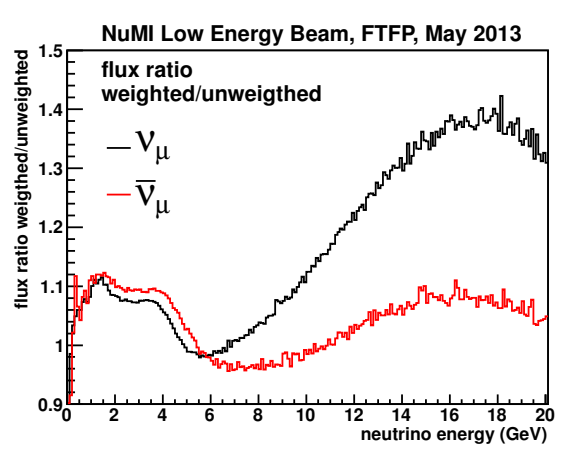

(b) Hadron Production Reweight Factors

Figure 4.2: Prediction of the flux (left) with hadron reweighting (right). This is the right sign flux, i.e. $\nu_{\mu}\left(\bar{\nu}_{\mu}\right)$ when NuMI focuses $\nu_{\mu}\left(\bar{\nu}_{\mu}\right)$.

\subsubsection{Resulting Flux}

The $\nu_{\mu}$ and $\bar{\nu}_{\mu}$ flux and hadron production reweight factors are shown in Fig. 4.2 .

\subsection{Neutrino Interactions}

MINERvA generated neutrino interactions with GENIE 2.6.42 [24 $]^{2}$ GENIE physics models that are relevant for MINERvA are valid in the "Medium-Energy Range" of $100 \mathrm{MeV}-$ $500 \mathrm{GeV}$, far wider than the energy spectrum of the NuMI beam. There are three steps to GENIE's simulation, each with its own family of models: nuclear physics, neutrino cross sections, and final state interactions (FSI). Each stage has been tuned to and/or validated against data, where available.

\subsubsection{Nuclear Physics}

Nuclei are modeled as a relativistic Fermi gas (RFG) [19]. The high momentum tails in the nucleon momentum distribution from the Bodek-Ritchie model are included. Pauli blocking

2 MINERvA actually used an off-brand version of GENIE tagged as R-2_6_4-minerva, which has identical physics to officially tagged 2.6.2 and 2.6.4 releases. The difference between 2.6.4 and 2.6.4-minerva is a trivial change which avoided the need for MINERvA to upgrade its version of ROOT. MINERvA also added code to record the full neutrino parentage back to the $p \mathrm{C}$ interaction, which was subsequently incorporated into the main GENIE branch. 


\begin{tabular}{ccc}
\hline Nucleus & $p_{F}$ for $\mathrm{p}(\mathrm{n})(\mathrm{MeV} / c)$ & $E_{b}(\mathrm{MeV})$ \\
\hline Carbon & $221(221)$ & 25 \\
Oxygen & $225(225)$ & 27 \\
Iron & $251(263)$ & 36 \\
Lead & $245(283)$ & 44 \\
\hline
\end{tabular}

Table 4.2: A summary of relativistic Fermi gas parameters in GENIE.

is implemented by disallowing quasi-elastic events which produce a nucleon in the final state that does not have a momentum greater than the Fermi momentum. The Fermi momentum $p_{F}$ and binding energy $E_{b}$ for relevant nuclei are listed in Table 4.2. See Sec. 1.3 .1 for further discussion on RFG.

The other nuclear models are relevant only for certain neutrino interaction processes and are discussed below, in the context of those interaction processes.

\subsubsection{Cross Section Models}

The first step in generating a neutrino interaction is to determine if and where a neutrino with $E_{\nu}$ (generated by NuMI simulation) will interact in the detector. This is done by calculating interaction probabilities based on the total neutrino-nucleus cross section at $E_{\nu}$, which is the sum of calculated cross sections for all interaction processes $p: \sigma\left(E_{\nu}\right)=\sum_{p} \sigma_{p}\left(E_{\nu}\right)$. GENIE's calculation of $\sigma\left(E_{\nu}\right)$ has been tuned to available data, as shown in Fig. 4.3 . After it has been determined that the neutrino interacts, GENIE selects the interaction process randomly by considering the relative likelihood for each process $P_{p}\left(E_{\nu}\right)=\sigma_{p}\left(E_{\nu}\right) / \sigma\left(E_{\nu}\right)$. Finally, event kinematics are determined according to the model of the particular interaction process.

\section{Quasi-Elastic Scattering}

Quasi-elastic scattering is modeled in the Llewellyn-Smith formalism described in Sec.1.2.1][5]. The vector form factors come from the BBBA05 parameterization [48]. The axial form factor 


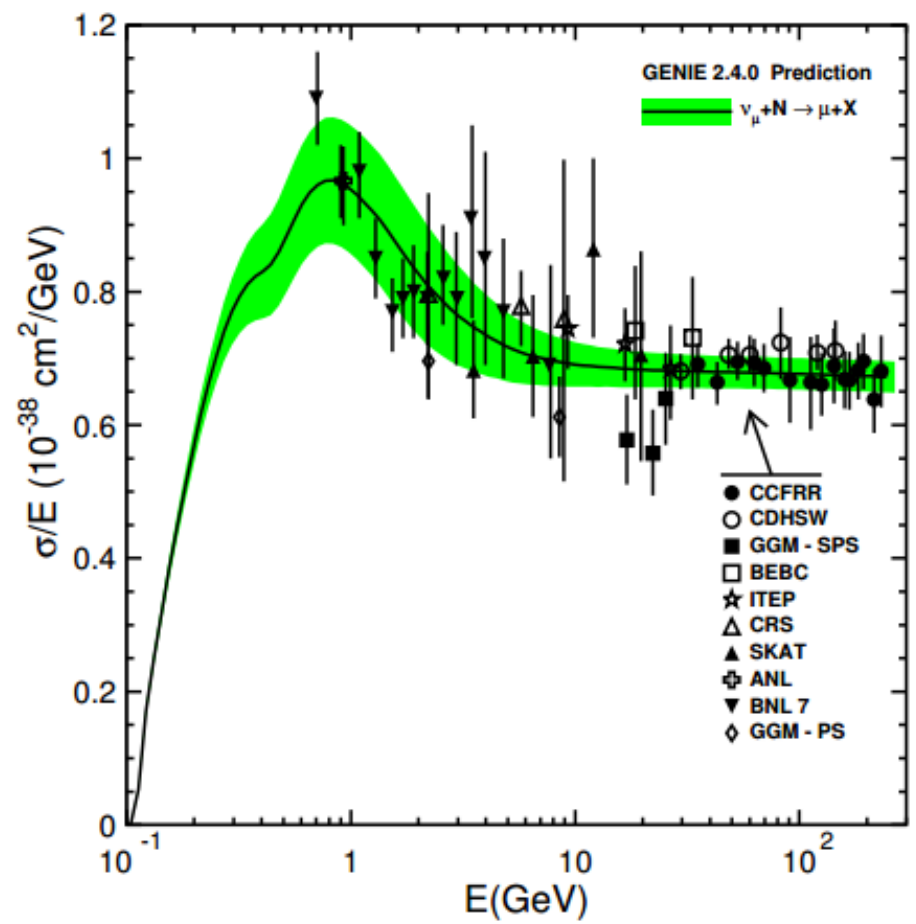

Figure 4.3: A comparison of GENIE's charged current inclusive NuMI cross section for an isoscalar target to world data. The shaded band is the uncertainty on free nucleon cross sections. Reprinted from [24], Copyright (2010) with permission from Elsevier. 
is modeled as a dipole with $M_{A}=1.01 \mathrm{GeV} / c^{2}$.

$$
F_{A}\left(Q^{2}\right)=\frac{F_{A}(0)}{\left(1+Q^{2} / M_{A}^{2}\right)^{2}}
$$

where $F_{A}(0)=-1.267$ is measured from neutron decay and the axial mass $M_{A}=0.99 \mathrm{GeV} / c^{2}$. Using the partially conserved vector hypothesis (PCAC) [49], the pseudo-scalar form factor is related to the axial form factor

$$
F_{p}=\frac{2 M_{n}^{2} F_{A}}{M_{\pi}^{2}+Q^{2}}
$$

Again, Pauli blocking in the RFG is implemented for quasi-elastic scattering, so the outgoing nucleon must have $p>p_{F}$.

\section{Resonance Production}

GENIE uses the Rein-Seghal model for neutrino-induced baryon resonance production [9]. Cross sections of 16 resonances are summed incoherently to obtain the total resonance production cross section. The resonances included are:

$$
\begin{aligned}
& P 33(1232), S 11(1535), D 13(1520), S 11(1650), \\
& D 13(1700), D 15(1675), S 31(1620), D 33(1700), \\
& P 11(1440), P 13(1720), F 15(1680), P 31(1910), \\
& P 33(1920), F 35(1905), F 37(1950), P 11(1710) .
\end{aligned}
$$

The P33(1600) and F17(1970) are calculated in Rein-Seghal model, but excluded in GENIE because they are ambiguous. The axial and vector masses are $1.12 \mathrm{GeV} / c^{2}$ and $0.84 \mathrm{GeV} / c^{2}$ respectively.

\section{Deep Inelastic Scattering}

GENIE's definition of DIS differs from the commonly used kinematic definition of $Q^{2}>$

$1(\mathrm{GeV} / c)^{2}$ and $W>2 \mathrm{GeV}$. In GENIE, all inelastic events that do not produce a resonance 
are treated as DIS. The DIS cross section is calculated in the Quark Parton Model. Parton distribution functions (PDFs) are modified according to the second iteration of a procedure by Bodek and Yang to account for higher twist and target mass effects through use of a new scaling variable $\xi_{w}$ [50]. Evaluation of PDFs is frozen at a $Q_{\min }^{2}=0.8(\mathrm{GeV} / c)^{2}$. The longitudinal structure function $R_{L}$ is the Bodek-Yang low- $Q^{2}$ modification to the Whitlow parameterization.

The Bodek-Yang procedure suggests modifications to nuclear structure functions in neutrino scattering [51]. These modifications are parameterizations of charged lepton DIS measurements for $F_{2}^{d} / F_{2}^{f r e e}$ and $F_{2}^{F e} / F_{2}^{d}$ as a function of $x_{b j}$, shown in Fig. 4.4. $F_{2}$ and $F_{3}$ for all nuclei larger than deuterium are modified according to this parameterization. That is, the nuclear PDFs for all nuclei are that of isoscalar iron. The DIS cross section in GENIE is scaled by 1.032 to match measured cross sections at $100 \mathrm{GeV}$. This scale factor is necessary because the Bodek-Yang nuclear modification does not attempt to treat the axial modification differently from the vector.
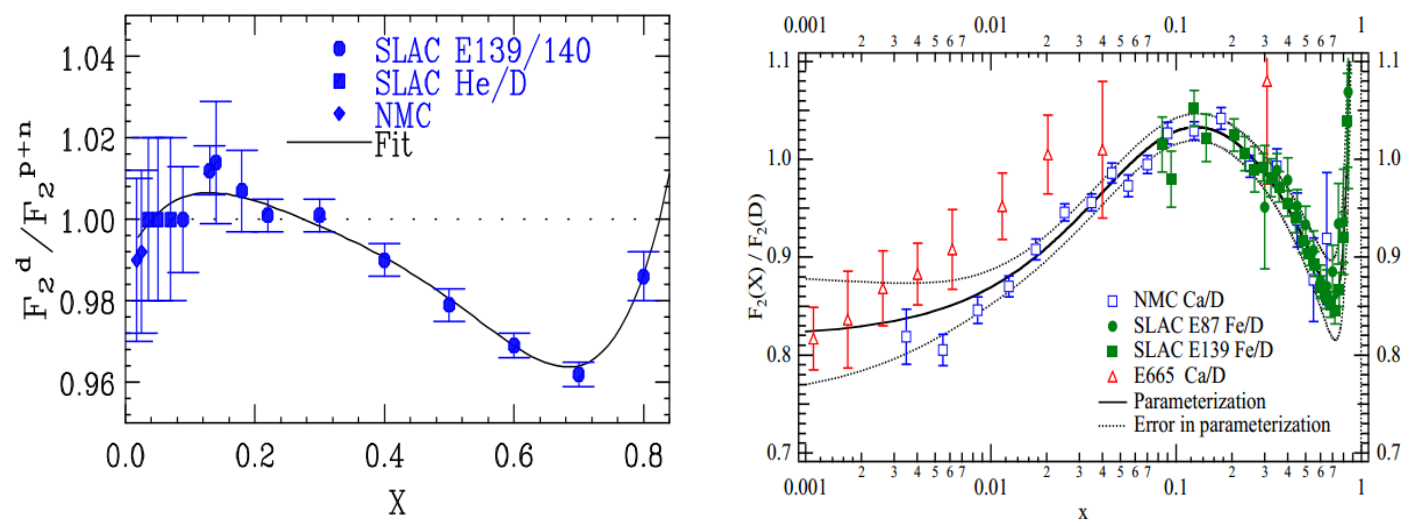

Figure 4.4: Bodek-Yang modifications to nuclear structure function $F_{2}$ as parametrization of charged lepton DIS data. (Left) Modification from free nucleon to deuterium. (Right) Modification from deuterium to isoscalar iron. Reprinted from [52], Copyright (2002) with permission from Elsevier. 


\section{Issues}

GENIE and the Bodek-Yang model were tuned using the leading order PDFs GRV981o. However, the MINERvA configuration of GENIE currently uses next-to-leading order PDFs GRV98nlo. This is a mistake that alters the total DIS cross section by as much as $5 \%$ and will be corrected for future MINERvA results. The GENIE predictions of the cross section ratios analysis presented here are not affected because there is no nuclear dependence in PDFs.

GENIE's implementation of Bodek-Yang has a bug due to improper use of scaling variables. In Bodek-Yang, the nuclear modification parameterization and low- $Q^{2}$ extension to $R_{w}$ are functions of $x_{b j}$. GENIE mistakingly uses $\xi_{w}$ for these calculations. The impact of the error on this analysis has been evaluated and is negligible due to the ratios technique.

\section{Transition from Resonance to DIS}

As mentioned in Sec. 1.2, the experimental distinction between resonance production and DIS is somewhat arbitrary. GENIE handles this disputed region by restricting resonance production to $W<W_{\text {cut }}$, where $W_{\text {cut }}=1.7 \mathrm{GeV}$. The DIS cross section is restricted to $W$ greater than the mass of the $\Delta^{++}\left(W_{\min }=M_{\Delta^{++}}=1.232 \mathrm{GeV}\right)$. The transition region is between $W_{\min }$ and $W_{c u t}$, where both DIS and resonance production occur. DIS interactions with resonance-like final states, meaning $1 \pi$ and $2 \pi$, are suppressed in this region to avoid double counting.

\section{Hadronization}

GENIE uses the AGKY hadronization model [53]. The model joins two descriptions of hadronization: KNO scaling and PYTHIA/JETSET [54, 55].

The AGKY use of KNO scaling is a phenomenological hadronization model developed for use in few-GeV neutrino scattering. It is relevant for lower values of $W$. First, the model selects the type and number of hadrons that will be generated using KNO scaling. Then, it assigns 
momentum to these hadrons by distributing the available $W$ among them and performing a phase space decay.

PYTHIA/JETSET is a comprehensive set of codes that is the standard for simulating high energy hadron collisions. It has a wide range of validity but is not tuned to neutrino-induced hadronization. Hadronization is calculated with the phenomenological Lund string fragmentation model [56].

AGKY uses KNO scaling for events with $W<W_{\min }=2.3 \mathrm{GeV}$ and PHYTHIA/JETSET for $W>W_{\max }=3.0 \mathrm{GeV}$. Between these bounds, either model for hadronization can be used. The probability of using KNO scaling goes from $100 \%$ at $W_{\min }$ to $0 \%$ at $W_{\max }$.

\subsubsection{Final State Interactions}

FSI in GENIE is performed by INTRANUKE, which is an intranuclear cascade (INC) simulation. INC models work by stepping hadrons through the nuclear environment, which is populated by nucleons. At each step, a hadron has a probability of interacting that is a convolution of the $h N$ cross section and the nuclear density. After it is determined that the hadron interacts, the interaction channel is selected randomly according to relative cross sections.

GENIE has two implementations of INTRANUKE. The $h A$ implementation considers the mean free path of hadrons in the nucleus to determine if and how FSI occur. The values for the mean free paths of the hadrons in the nucleus come from a wide variety of experiments. This model has been extensively compared with data for validation. A newer model $h N$ considers interactions with nucleons in the nuclear environment, and is thus a better representation of INC. The $h A$ model was used in the analysis presented here, but MINERvA will use the $h N$ model in the future because it has been shown to give a more accurate description of FSI in neutrino interactions. 


\subsection{MINERvA Simulation}

MINERvA's observation of neutrino interactions in the detector is simulated in two stages. The first is the propagation and energy loss of the final state products of neutrino interaction. The second is the collection and interpretation of energy deposited into active materials. To make the simulation match data as accurately as possible, generated neutrino events are overlaid onto beam data gates.

\subsubsection{Propagation of Particles in MINERvA}

Geant4 9.4.p02 is used to simulate the propagation of particles through the mass of the MINERvA detector [57]. The simulation works by propagating particles in time steps, then determining if and how they interact with the material. Hadron physics is simulated with the QGSP_BERT module, which uses a Bertini model for intranuclear cascade for hadrons with energies below $10 \mathrm{GeV}$. Nearly all final state hadrons in this analysis are less energetic than $10 \mathrm{GeV}$, and QGSP_BERT outperforms other modules here because quark gluon string calculations are not expected to be as effective at these lower energies. The standard Geant 4 modules are used for all other processes.

\subsubsection{Overlaying Simulated Events onto Data Gates}

Many aspects of the data are not simulated. These include event overlap in the detector, events in the calorimetry, rock muon events, dead time, dead channels, and mis-calibration. Instead of developing complicated extensions to the simulation, the effects are directly imported from data. The data overlay procedure is preferable as it is clearly more representative of the data than any simulation of these effects could be.

Each generated neutrino event is paired with one gate that comes from the run period being modeled. Only gates that contain more than $5 \times 10^{12}$ P.O.T. are used, because empty spills are not analyzed. Hits and beam spill information from the gate are used throughout the rest of 
simulation. When analyzing a Monte Carlo with overlaid data, it is important not to consider overlaid data as signal candidates.

\subsubsection{MINERvA Readout and Electronics}

The neutrino interaction is placed at a time within the spill according to distribution of NuMI beam's batch structure. An optical model translates the energy deposited in scintillator into light using Birks' law [58]. Decalibrations are applied so that the photostatistics and light yield in the simulation match those found in data. Time-dependent calibrations applicable to the overlaid data event are used to decalibrate the energy and include the identification of dead channels. A PMT model that includes optical cross-talk converts the light into charge. The time it takes for light to propagate through optical fibers is smeared with a data-driven model. Digitization of charge into hits is simulated by a model of the front end electronics. Simulated hits are checked against the dead time found in the overlaid data gate and masked accordingly.

\subsection{MINOS Simulation}

The positions and momenta of simulated particles that exit the back from MINERvA are fed into a MINOS -owned GEANT3 simulation of the MINOS near detector [59]. The simulation includes the passage of charged particles through the magnetic field and the readout of energy deposited in active elements. Reconstruction is then performed using the hits generated by these simulated particles.

Hit and track information is retained from the MINOS gate (called a "snarl") that corresponds to the MINERvA gate used in the data overlay procedure. In doing so, the confusion during the process of matching a reconstructed track from MINERvA into MINOS that occurs due to event overlap is simulated. Note that overlap during track finding in MINOS is not simulated, because the reconstruction only considers the hits on generated particles. 


\title{
Chapter 5
}

\begin{abstract}
Analysis
This chapter presents an analysis of the ratios of the charged current inclusive cross section on carbon, iron, and lead to scintillator. The analysis is restricted to interactions with $2 \mathrm{GeV}<$ $E_{\nu}<20 \mathrm{GeV}$ and $0^{\circ}<\theta_{\mu}<17^{\circ}$, due to the limitations of the MINOS spectrometer. The event selection allows scintillator events to contaminate the carbon, iron, and lead samples. This scintillator background is subtracted using a data-based procedure. The measured ratios of cross sections and absolute cross sections are calculated, along with the sources of uncertainty on this measurement. This analysis is performed using $2.94 \times 10^{20}$ P.O.T. of $\nu_{\mu}$-mode data recorded by MINERvA from 2009-2012. The results of this analysis are shown and discussed in Chapter 6 .
\end{abstract}

\subsection{Selection of Charged Current $\nu_{\mu}$ Events}

A charged current neutrino event produces a charged lepton in the final state. This analysis requires the presence of a $\mu^{-}$, the signature of a $\nu_{\mu}$ scattering, with no requirement on the composition of the hadronic system.

$$
\nu_{\mu}+A \rightarrow \mu^{-}+X
$$

The interaction nucleus is identified by the position of track-based vertex reconstruction. Data quality cuts ensure the reliability of the reconstruction. 


\subsubsection{Muon Requirements}

This analysis identifies charged current events as those which contain exactly one muon. A muon is identified as a tracked particle that exits the back of MINERvA, and is chargeand momentum-analyzed in the MINOS near detector. The requirements for matching a track in MINERvA to a track in MINOS are described in 3.2.6. Muons that stop in MINERvA are difficult to differentiate from pions and are not used in this analysis. Muons that exit MINERvA and are not matched to a reconstructed track in MINOS are not used, because the muon energy can only be reconstructed if the particle either stopped or was measured by its curvature in a magnetic field. The muon charge must be negative, which is accomplished by selecting events in which the MINOS track has a negative curvature, $\frac{q}{p}<0$.

\section{Effects on Acceptance}

The requirements to match a track into MINOS restrict the explored kinematic space due to the geometrical relationship of MINERvA to MINOS and the tracking threshold of MINOS.

Because MINERvA is located upstream of MINOS, muons with larger scattering angles are less likely to enter MINOS. Vertices that are farther upstream (i.e. farther from MINOS) have a lower angular threshold for acceptance. The angle of accepted events is restricted to $17^{\circ}$ with respect to the beam direction. This ensures adequate acceptance to perform measurements in the most upstream nuclear targets.

The muon must have enough momentum to travel through MINERvA, enter MINOS, and traverse enough MINOS planes to form a track. The momentum required to exit MINERvA depends on how close the vertex is to the back of MINERvA and ranges from 0.2 to $1.2 \mathrm{GeV} / c$. Muons that originate near the upstream region of the detector will lose more energy through $d E / d x$ processes and thus have a higher momentum threshold. The MINOS tracking efficiency for muons entering the front plane of MINOS is shown in Table 5.1 .

Only events with $2<E_{\nu}<20 \mathrm{GeV}$ are used in this analysis. The lower limit implies 


\begin{tabular}{c|ccccccc}
\hline Momentum Bin Low Edge $(\mathrm{GeV} / c)$ & 0.00 & 0.15 & 0.25 & 0.50 & 0.75 & 1.00 & 1.25 \\
Efficiency & 0.00 & 0.55 & 0.60 & 0.60 & 0.80 & 0.90 & 1.00 \\
\hline
\end{tabular}

Table 5.1: Approximation of MINOS tracking efficiency for muons that enter the front of MINOS as a function of momentum. Efficiency is the fraction of muons entering the detector that are tracked [60].

a minimum momentum for the muon, which ensures adequate acceptance. Cutting directly on the muon momentum is not done because it would complicate the calculation of cross sections as a function of the neutrino energy. The upper limit is used because a selection of very high energy $\nu_{\mu}$ events will have a larger contamination of $\bar{\nu}_{\mu}$ events. This decrease in $\nu_{\mu}$ purity occurs because the flux at these higher energies consists of many neutrinos produced by the decay of unfocused mesons, which have not been charge-selected.

\subsubsection{Fiducial Volume and Target Identification}

The event vertex must be within a regular hexagonal area with apothem $850 \mathrm{~mm}$. This ensures that the event did not occur in the lead of the electromagnetic calorimetry that surrounds the perimeter of the detector. The areal cut also restricts the fiducial area to a region where the acceptance into MINOS changes less rapidly than at the outer edges of the detector. The requirements for selecting events in the passive and active targets are slightly different due to inherent differences in reconstruction.

\section{Scintillator Sample}

Scintillator events are those with a vertex in the fully active central tracking region. The fiducial volume consists of modules $27-80$, inclusive. The full tracking region spans modules 23-84. The limitation of the fiducial region to modules $27-80$ provides a sufficient buffer to eliminate events originating in the iron and lead of the passive targets region (upstream of tracker), and lead of the electromagnetic calorimetry (downstream of tracker). 


\section{Passive Target Samples}

Events in the passive target samples must pass two stages of vertex cuts. The first cut is on the longitudinal position, which identifies the target assembly (Targets 1-5) of the interaction. The second cut is on the transverse position, which identifies the nucleus within the target assembly with which the interaction occurred.

\section{Target Assembly Identification}

Energy deposited in passive targets cannot be directly observed. For events originating in a slab of passive material, the first signal measured is from the energy deposited in the first scintillator module downstream of the passive target. If there is only one reconstructed track, an event from the passive material and an event in the first module downstream of the passive material cannot be distinguished. Events that have a vertex in the module downstream of a passive target are assumed to be from the passive target. An example event display of a carbon event candidate with one track is shown in Fig. 5.1. Because the passive targets are more dense than a scintillator module, this assumption is usually correct. This selection also allows interactions actually in the scintillator, and the procedure for subtracting the contribution from these scintillator events is described in Sec.5.3. The muon track is projected along its trajectory to the longitudinal center of the target assembly using a Kalman filter [40]. The projection identifies where the event would have occurred if indeed it occurred in the target assembly. The muon energy is adjusted using $d E / d x$ for the material traversed according to the propagation.

Events with more than one reconstructed track can be fit to find a three-dimensional vertex, which may be inside the passive material. An example event display of a lead event candidate is shown in Fig. 5.2. However, the resolution of the vertex reconstruction is not sufficient to prevent smearing of scintillator interactions into passive material, and vice versa. The same selection criteria are applied to events with fitted vertices and one track events. Any gains in

purity by applying a stricter criterion would be more than compensated by loss of efficiency, 


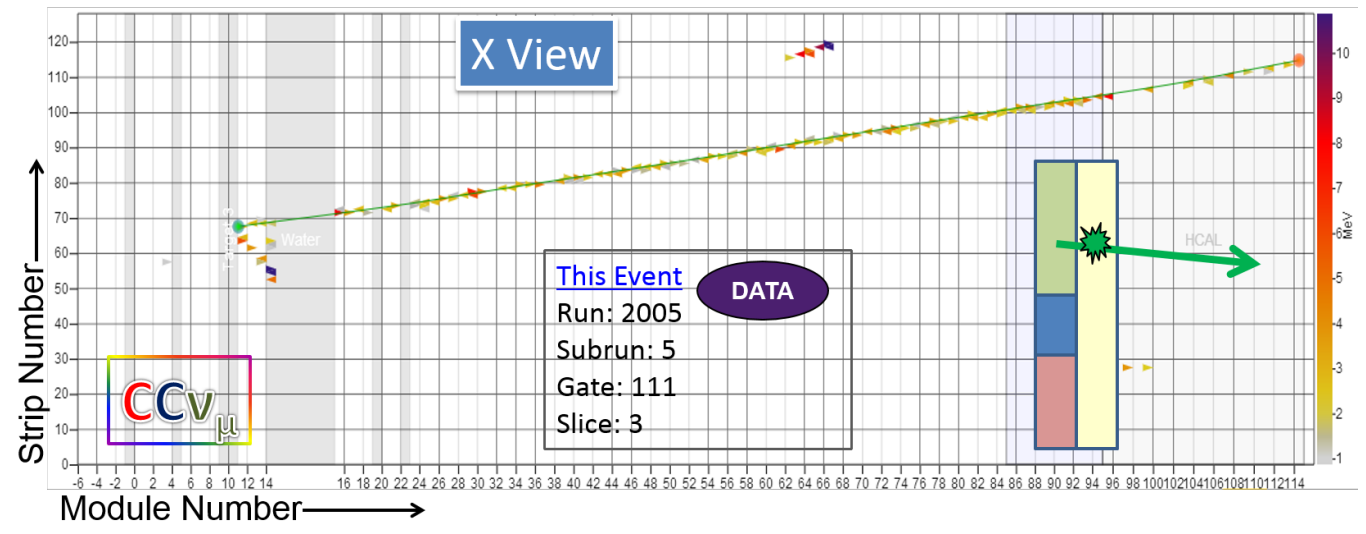

Figure 5.1: A carbon event candidate that has only one reconstructed track. The beam enters from the left and MINOS sits to the right of the event display. Passive targets are shown as shaded rectangles in the upstream region of the display. Only the $\mathrm{x}$ view is shown. The reconstructed vertex is in the first plane downstream of the passive carbon target. A cartoon of the event is inset on the lower right of the event display.

because there are a significant number of interactions in passive material whose vertex is reconstructed in scintillator.

The selection criterion for passive target events is that the vertex is between one plane upstream and one module downstream of the target. The selected event sample of each target is roughly $70 \%$ one track, $10 \%$ fitted vertices in the target, $20 \%$ multi-track events that do not have a vertex in the passive target [61].

\section{Target Nucleus Identification}

The transverse position of the vertex is compared with the orientation of the carbon, iron, and/or lead of the target assembly. e.g. if the vertex is in the area occupied by iron, the event goes into the iron sample.

The vertex is required to be more than $2.5 \mathrm{~cm}$ away from any seams between the passive materials (e.g. where iron meets lead). There is welding and some overlap of different materials at the seam, which would lead to ambiguity in the assignment of interaction nucleus. This requirement also serves to reduce the number of events with misidentification of interaction nucleus due to transverse smearing to the point where this background is negligible. 


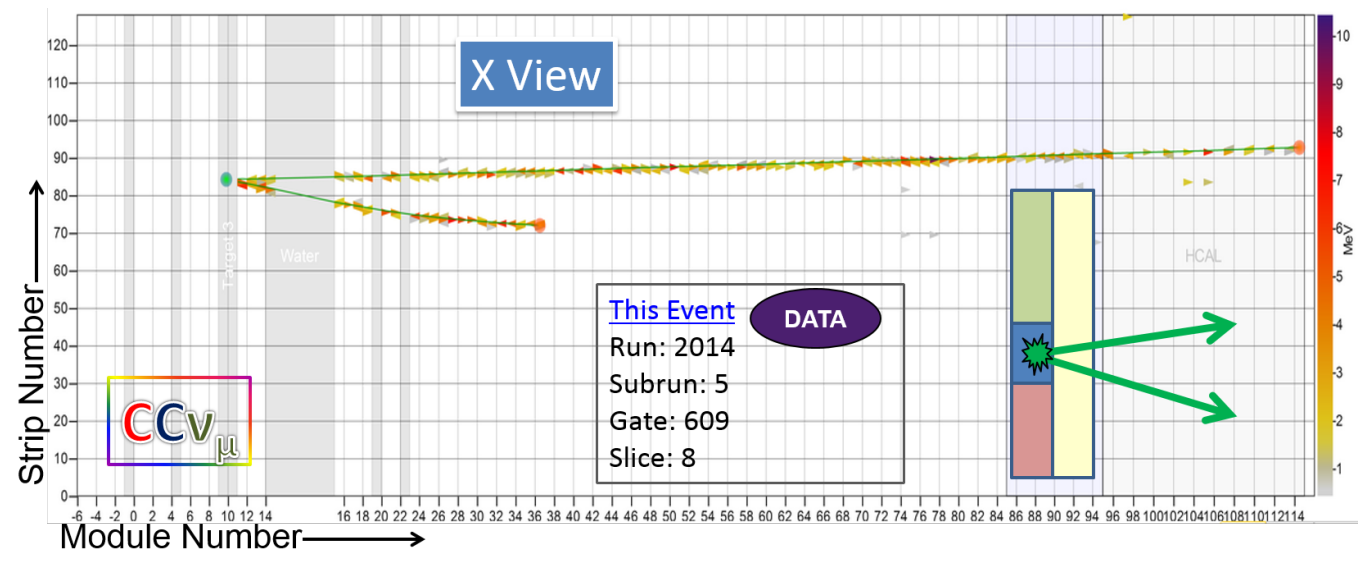

Figure 5.2: A lead event candidate that has two reconstructed tracks that have been fit to a 3D vertex in the lead. Figure 5.1 gives a general description of the event display.

\begin{tabular}{ccccc}
\hline Nucleus & Mass (ton) & N Protons (E30) & N Neutrons (E30) & N Nucleons (E30) \\
\hline Carbon & 0.159 & 0.048 & 0.048 & 0.096 \\
Iron & 0.628 & 0.176 & 0.203 & 0.379 \\
Lead & 0.711 & 0.169 & 0.258 & 0.427 \\
Scintillator & 5.476 & 1.760 & 1.534 & 3.294 \\
\hline
\end{tabular}

Table 5.2: Mass and number of scatterers in the fiducial volume of each target material.

\section{Scintillator Faux Targets}

The passive nuclear targets are segmented in the transverse direction into different materials, as shown in Fig. 2.8. Throughout this analysis, sections of tracker are used as references for these target sections. A faux target is defined as the scintillator contained in a group of 6 contiguous tracker modules within a transverse region similar to the passive target section being referenced. For example, one faux target for the iron of Target 2 is the transverse area of modules 27-32 that resembles the iron of Target 2. The first faux target begins with module 27; the last ends with module 80 .

\section{Number of Scatterers}

Table 5.2 shows the mass and target number of the fiducial regions. 


\subsubsection{Reconstruction Quality Assurance}

Correct identification of the interaction nucleus is paramount for the success of this analysis. To ensure that the reconstruction of the vertex is reliable, events that have characteristics of poor reconstruction are rejected. Interactions in the detector may be reconstructed poorly due to detector problems or algorithmic shortcomings. Interactions external to the detector, notably "rock muons" which are defined below, may be incorrectly identified events in the fiducial region.

\section{Rejecting Bad Vertex Reconstruction}

Proper tracking of the muon is the most critical step in vertex reconstruction. The muon track may be obscured by hadronic activity, which can cause hits generated by the muon near the vertex to fail to be associated with the muon track. In such events, the muon is tracked starting from the downstream end and stops short of reaching the interaction vertex. A similar topology occurs when one or more scintillator planes traversed by the muon fails to record hits. Readout inefficiency may happen due to dead time or dead channels. The procedure for identifying all of these problems is to look for muon-like activity that is upstream of the vertex. Events were rejected if they fit any of the following criteria:

- Hits were recorded in eight or more planes in the muon's projected upstream path, and the average energy of the hits was between 1.5 and $6.5 \mathrm{MeV}$ per plane.

- Hits were recorded in six or more consecutive planes in the muon's projected upstream path, and the average energy of the hits was above $1.5 \mathrm{MeV}$ per plane.

- More than one TriP-t associated with strips upstream of the muon track were dead. This indicates that some activity associated with the track may not have been observed. 


\begin{tabular}{c|cccc}
\hline Target & N Carbon & N Iron & N Lead & N Scintillator \\
\hline 2 & - & 8190 & 6624 & - \\
3 & 5953 & 4001 & 2390 & - \\
4 & - & - & 10245 & - \\
5 & - & 6833 & 4708 & - \\
Tracker & - & - & - & 189168 \\
Total & 5953 & 19024 & 23967 & 189168 \\
\hline
\end{tabular}

Table 5.3: Neutrino events selected in the carbon, iron, lead, and scintillator charged-current sample.

\section{Rock Muon Rejection}

Rock muons are the final state muons of neutrino events external to the MINERvA detector. They are produced when neutrinos interact in the rock of the cave or the equipment in the detector hall upstream of MINERvA. Often these rock muons enter the front or side of the detector and exit the back, where they may continue into MINOS. Most beam spills produce several rock muons that enter the detector. These muons match the event selection criteria for the charged current inclusive sample, except the criteria associated with the vertex position. The quality assurance cuts defined above in Sec. 5.1.3 also serve to remove the majority of poorly reconstructed rock muon events.

\subsection{Event Sample}

The number of events in the charged current samples is listed in Table 5.3. Kinematic and vertex distributions of selected events are shown in Appendices A.1 and A.3, respectively.

\subsection{Backgrounds}

There are three types of background in the event sample.

- Neutrino events of the wrong channel. These include wrong sign $\left(\bar{\nu}_{\mu}\right)$ events and neutral current events. 
- Rock muons that were not removed by quality assurance cuts.

- Events reconstructed in the wrong nucleus.

\subsubsection{Wrong Sign and Neutral Current Events}

A wrong sign (WS) event can be falsely accepted in the event sample when a $\bar{\nu}_{\mu}$ chargedcurrent interaction produces a muon whose charge is reconstructed as negative. Although a $\bar{\nu}_{\mu}$ event can lead to a $\mu^{-}$through charm production, the vast majority of the WS background is the result of a reconstruction failure in MINOS. Even before charge-identification, the WS background is very small because the great majority of events are $\nu_{\mu}$ interactions due to the flux being predominately $\nu_{\mu}$ 's and the fact that the $\nu_{\mu}$ cross section is roughly twice that of the $\bar{\nu}_{\mu}$ cross section. The WS contamination is smallest near the flux focusing peak, where the charge selection of mesons in the beamline is most effective. This background is very small $(<0.4 \%$ ) .

Neutral current (NC) events do not produce a charged lepton in the final state. It is possible for final state products to decay into a muon that enters MINOS, but most of the NC background is due to reconstruction failures. There are a small number of high energy pions that exit MINERvA and form a track in MINOS before losing their energy in a hadronic shower. The other source of NC background is incorrect matching of a MINERvA to MINOS track. For example, a final state pion is tracked to the back of MINERvA and perhaps exits. This pion track may be coincident with and incorrectly matched to an unrelated muon track in MINOS. The NC background also is very small $(<0.1 \%)$.

Both of these backgrounds are estimated using the Monte Carlo predictions for their fractional contributions. Figure 5.3 shows the Monte Carlo prediction for the fraction of the Target 4 lead event sample that comes from WS and NC events. The NC and WS backgrounds are shown for all targets in Appendix A.2. 

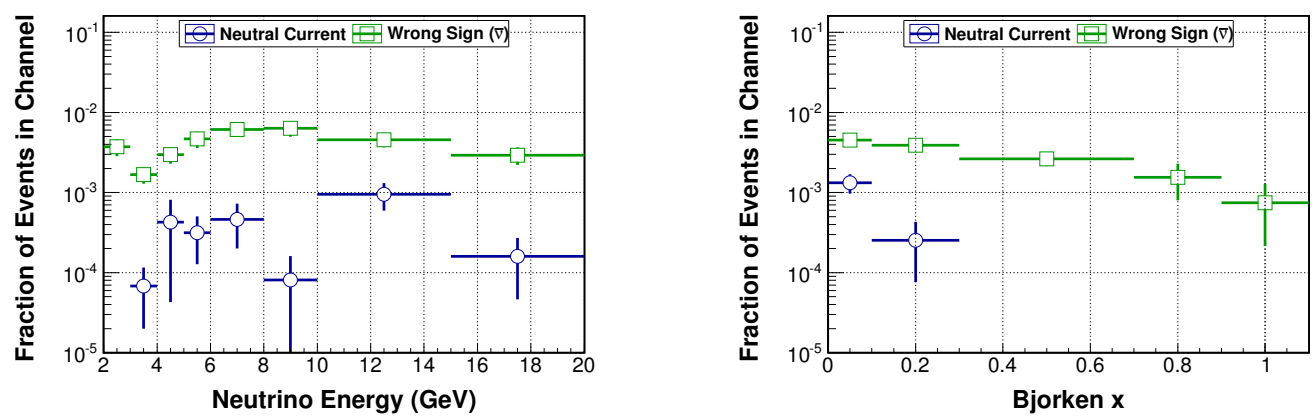

Figure 5.3: Monte Carlo prediction for the fraction of neutral current and wrong sign events in the lead sample from Target 4 .

\subsubsection{Rock Muon Events}

The two most upstream tracking modules of the detector were not instrumented in the earliest data taking period, because electronics were more urgently needed to service the test beam detector at that time. This diminished the rock muon veto capabilities of the detector in the most upstream region only. Further compounding the problem, there were several regions of dead channels near Target 2 in data taken between May 22, 2010 and July 12, 2010, which lowered the tracking efficiency near that target. The scale of the contamination was determined through a team event scan effort. The scan revealed that 60 out of $975(6.2 \pm 0.8 \%)$ events were rock muon contamination of the sample for Target 2 in the data recorded in playlist 1 . No other regions of the detector or playlists showed significant contamination. There is no statistically significant difference in the kinematic distributions of these rock muon events and signal events. A flat weight of $1-.062=.938$ is applied to events found in Target 2 in the data from playlist 1.

\subsubsection{Misidentified Nucleus}

In order to understand the nuclear dependence of neutrino interactions, the event samples must be separated by interaction nucleus as well as possible. The event selection for passive 
target samples is designed to allow some contamination from scintillator. The fractional contribution of the scintillator background is roughly proportional to the ratio of areal mass of the passive target to nearby scintillator, which has a range of $20-40 \%$. This is a significant background and is subtracted by a procedure described in Sec.5.4. The number of events migrating from other passive targets is small $(<1 \%)$ and is subtracted using the Monte Carlo prediction for the fractional contribution of these events to the passive target event sample. Figure 5.4 shows Monte Carlo predictions for the backgrounds from misidentified nucleus for the iron of Target 2. The background scales are similar for other passive targets, except that the fractions vary with the mass of the signal target.

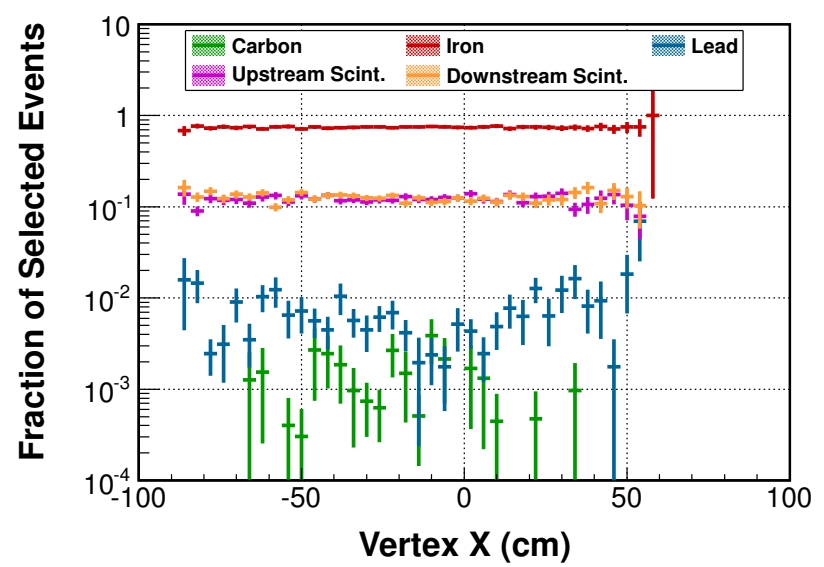

Figure 5.4: Monte Carlo prediction for the fraction of events in the Target 2 iron sample that has a true nucleus of interaction in carbon (green), iron (red), lead (blue), and scintillator (purple and orange).

The scintillator has no background due to misidentified nuclei, because the fiducial volume is very large and well separated from other materials. Scintillator is a composite material that is predominately carbon and hydrogen. It is not possible to separate events based on the interaction nucleus in scintillator, so it is treated as a composite material. 


\subsection{Scintillator Contamination Subtraction}

By design, the event selection procedure allows events in scintillator into the passive target event samples. This scintillator contamination is shown in orange as scintillator contaminating the passive target samples Fig. 5.5. This contamination must be subtracted in order to measure the cross section of a specific nucleus found in the passive target. The subtraction procedure does not rely on a cross section model; the measured event rate in the tracker fiducial volume is used to predict the spectrum for the scintillator impurity. The tracking and nuclear target regions occupy different areas, and, therefore, have different acceptance into MINOS. The data-driven background subtraction procedure described below uses efficiency and acceptance corrections from two separate Monte Carlo samples to minimize systematic uncertainties. The steps of the procedure are as follows:

- First, a sample of scintillator events is selected from the data using the procedure described in Sec. 5.1

- Next, the difference in geometric acceptance between events in the tracking region and events in the passive targets is accounted for using a muon-only Monte Carlo.

- Then, efficiency differences that depend on the hadronic energy are accounted for using simulated neutrino events to derive an efficiency correction.

- Finally, the efficiency-corrected distribution is scaled so that the integrated number of events in the true background and predicted background are equal.

\subsubsection{Geometric Acceptance Correction}

Events in the tracker region and events in the passive targets region have different geometric acceptance due to their different positions relative to MINOS. The geometric acceptance is calculated using a muon-only simulation implemented with Geant4. Acceptance is defined 


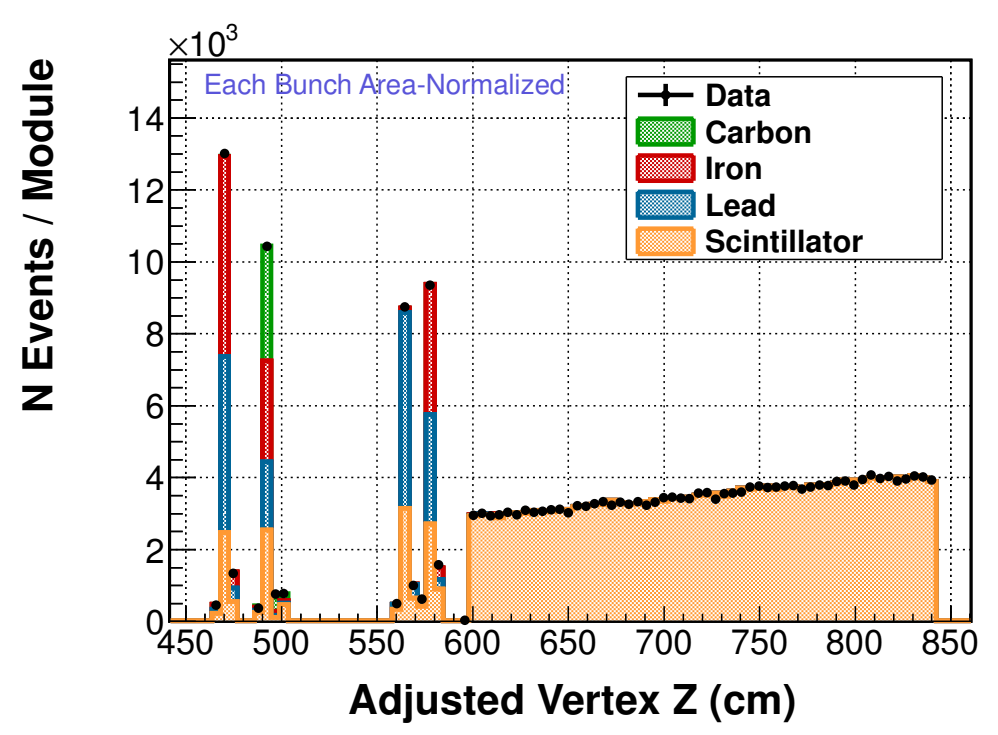

Figure 5.5: Distribution of the $\mathrm{z}$ coordinate of the vertex showing misidentification of the interaction nucleus. Data is shown in black points with statistical error. The Monte Carlo event sample is shown as stacked histograms which are color-coded by the true interaction nucleus. Passive carbon, iron, and lead targets are located at the large spikes in the nuclear targets region $(450-600 \mathrm{~cm})$. The upward slope throughout the tracker region $(600-840 \mathrm{~cm})$ is an effect of the acceptance in MINOS. The number of Monte Carlo events in each target has been scaled to the number of data events.

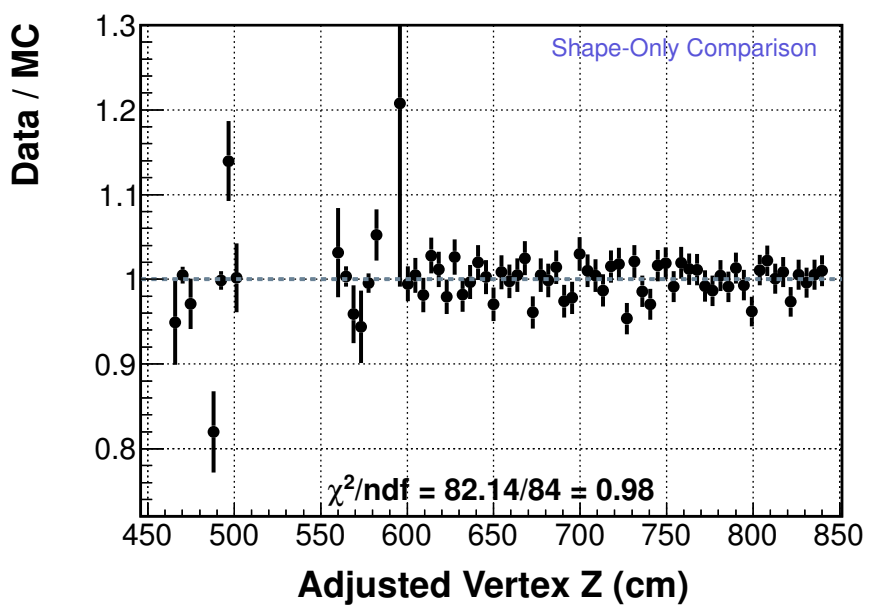

Figure 5.6: Ratio of vertex-Z distribution in data to simulation using the histograms of Fig. 5.5 
as the fraction of muons with a given energy, angle, and position that enter MINOS in a reconstructible state. The acceptance in calculated at points every $200 \mathrm{~cm}$ in the longitudinal direction, $5 \mathrm{~cm}$ in the transverse directions, $0.2 \mathrm{GeV}$ in muon energy, and $1^{\circ}$ in muon polar angle. At each of these points, three muons are generated at each degree of $\phi$ for a total of 1080 muons. Each of these muons is tested for acceptance using the following criteria based on the true path of the muon in the simulation:

- The muon must reach the back of MINERvA within the area of regular hexagon with apothem $1.07 \mathrm{~m}$ (inner detector size).

- The muon must enter MINOS in the "partially instrumented" calorimeter region and stay in that area for $60 \mathrm{~cm}$, which is long enough to be tracked.

- The muon must have sufficient momentum when it enters MINOS. Instead of a binary decision, a MINOS tracking efficiency weight is used. This efficiency curve is shown in Table 5.1

The fraction of muons that are accepted, weighted for MINOS tracking efficiency, is the acceptance for that point. The acceptance is shown as a function of muon energy and angle for two longitudinal positions is shown in Fig. 5.7 .

To reduce statistical fluctuations, the acceptance is integrated over each target section (e.g. iron of Target 2) and each faux target section (e.g. transverse area of modules 27-32 resembling iron of Target 2). The acceptance corrections are then a function of the muon energy and angle, and of the position of the target or faux target section containing the vertex. Each event in the tracker is weighted as

$$
w=\frac{f_{\text {acc }}^{t g t 2, F e}\left(E_{\mu}, \theta_{\mu}\right)}{f_{a c c}^{f a u x 2, F e}\left(E_{\mu}, \theta_{\mu}\right)},
$$

where $f_{a c c}^{t g t 2, F e}$ is the fraction of muons accepted in the iron of Target 2 and $f_{\text {acc }}^{f a u x 2, F e}$ is the fraction accepted in the faux target for that section. 


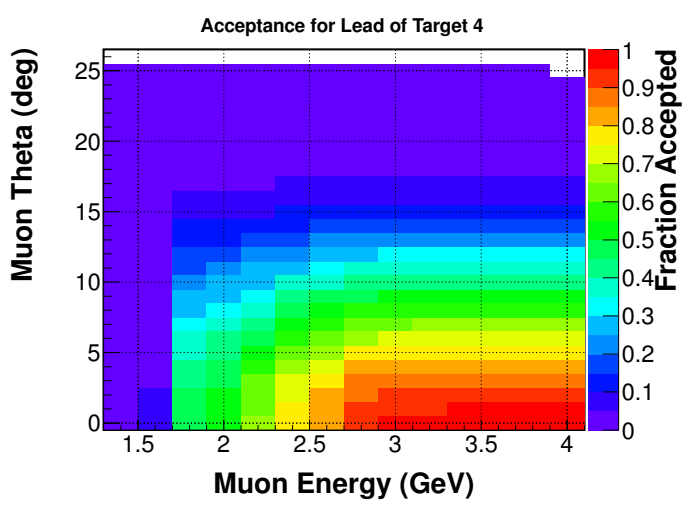

(a) $6.4 \mathrm{~m}$ from MINOS

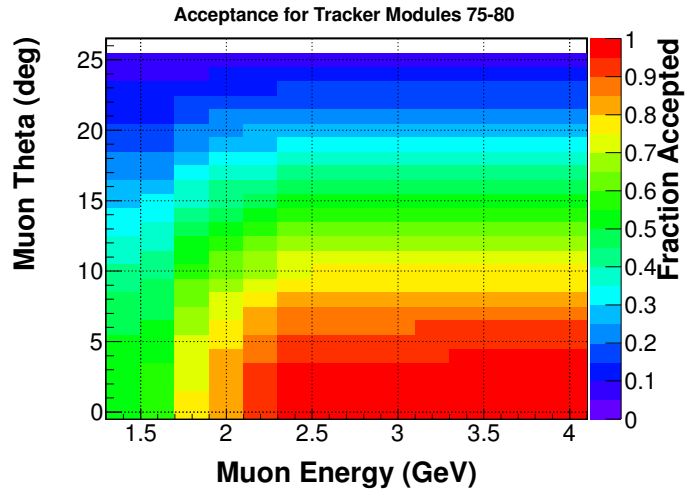

(b) $3.4 \mathrm{~m}$ from MINOS

Figure 5.7: Muon acceptance as a function of energy and angle shown for two longitudinal regions of the detector. Acceptance falls as low energy because the muons lack the momentum to make it through the material of MINERvA and into MINOS. Acceptance falls at high angle because the muon is less likely to point to MINOS. Both effects are more dramatic for upstream events.

The procedure for estimating the distribution of background events from scintillator is as follows:

1. Events are selected in the faux targets corresponding to the passive target. Kinematic distributions are created using these events and weights from Eq. 5.2. This is done for both data and simulated samples.

2. The distribution in simulation is scaled to the true background, so the integral number of events is equal. The same scale factor will be applied to the data prediction of the background.

\subsubsection{Hadronic Energy Efficiency Correction}

Predictions using only the geometric acceptance fail to match the true background in certain variables, most noticeably at low $E_{h a d}$, because the reconstruction efficiency of neutrino events depends on more than just the muon kinematics. For example, events with more hadronic energy will have a lower reconstruction efficiency because the shower may obscure the muon 

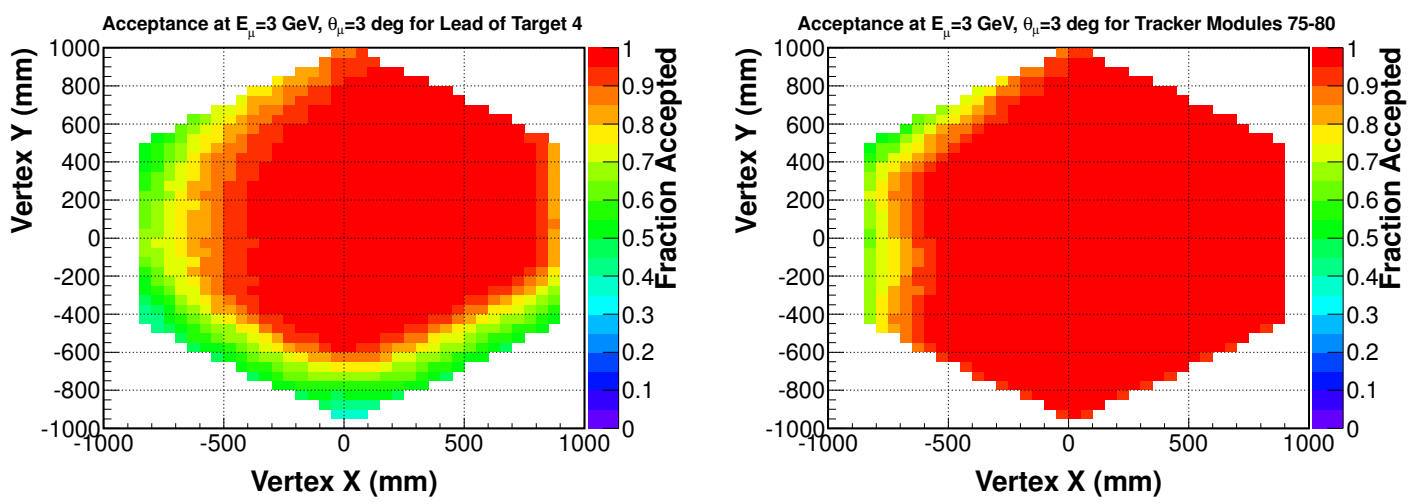

(a) $\theta_{\mu}=3^{\circ}, 6.4 \mathrm{~m}$ from MINOS

(b) $\theta_{\mu}=3^{\circ}, 3.4 \mathrm{~m}$ from MINOS
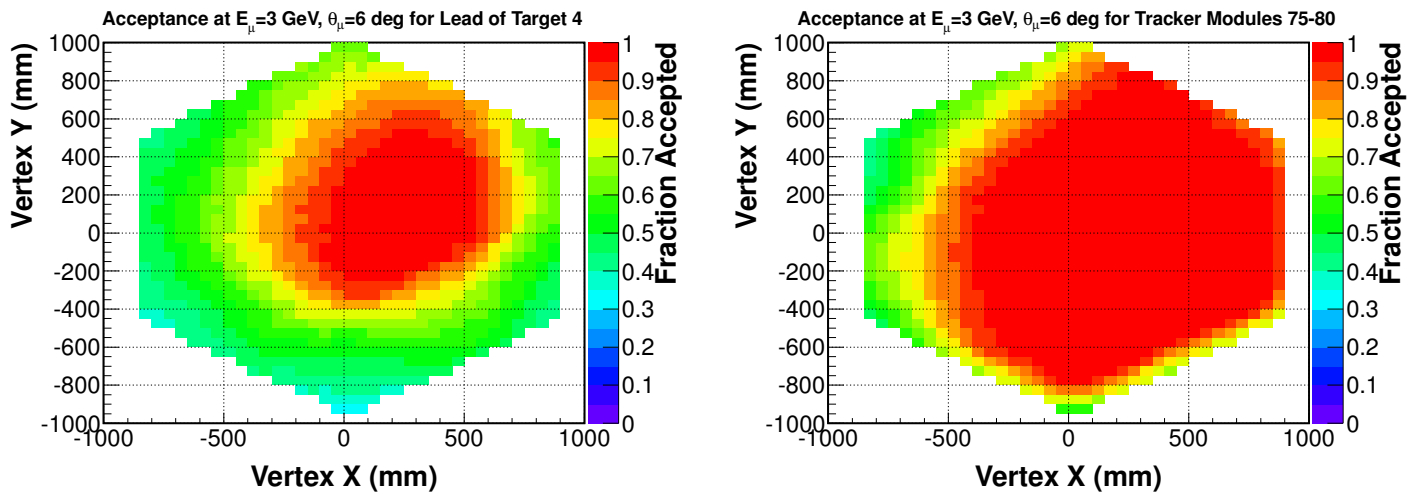

(c) $\theta_{\mu}=6^{\circ}, 6.4 \mathrm{~m}$ from MINOS

(d) $\theta_{\mu}=6^{\circ}, 3.4 \mathrm{~m}$ from MINOS

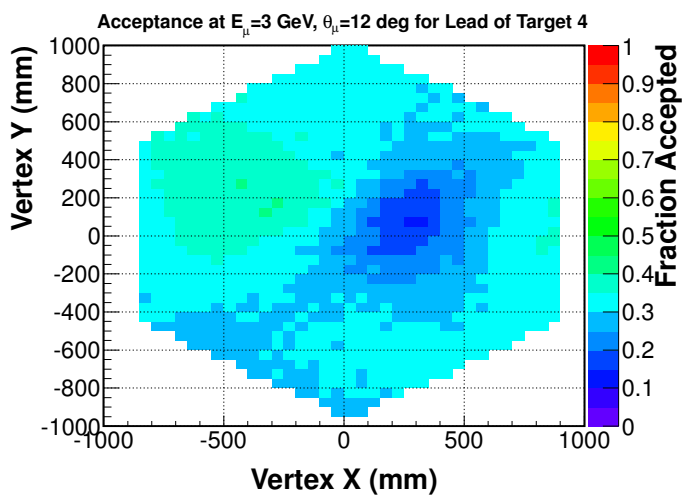

(e) $\theta_{\mu}=12^{\circ}, 6.4 \mathrm{~m}$ from MINOS

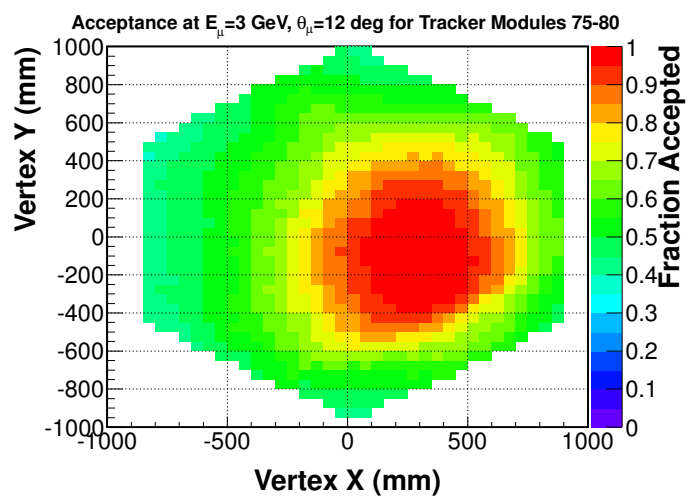

(f) $\theta_{\mu}=12^{\circ}, 3.4 \mathrm{~m}$ from MINOS

Figure 5.8: Muon acceptance as a function of transverse position shown for two longitudinal regions of the detector and three angles. All muons in the plot have an energy of $3 \mathrm{GeV}$. Acceptance falls in regions that are far from the MINOS fiducial area, which is generally the outer edges of MINERvA. The fiducial region of MINOS is most apparent in the plots at $\theta_{\mu}=$ $6^{\circ}$ in the upstream region (middle left) and $\theta_{\mu}=12^{\circ}$ in the downstream region (lower right). 


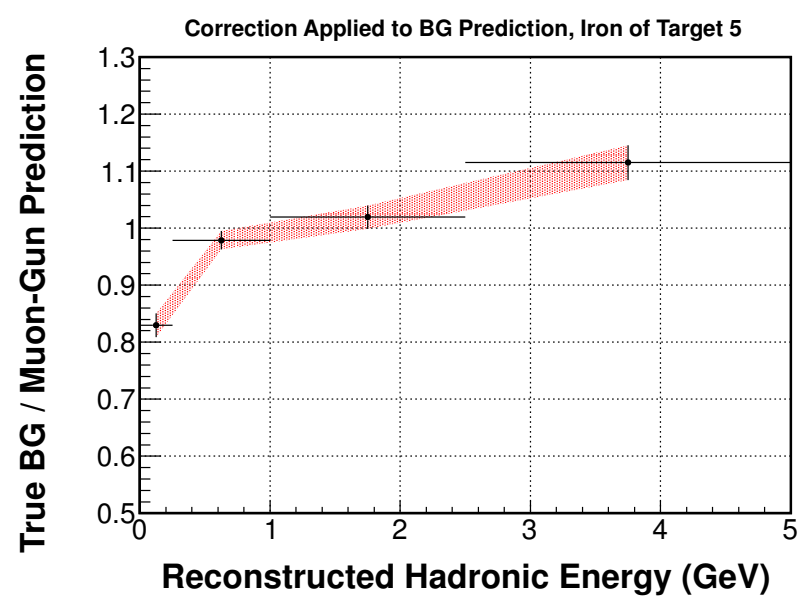

Figure 5.9: $E_{h a d}$-dependent scale factor in prediction of background from scintillator in passive targets in the iron of Target 5 . The shaded band shows the statistical uncertainty.

track. This effect is larger near passive targets, because the heavy nuclei stimulate electromagnetic and hadronic showers. To obtain an acceptably accurate prediction of the background, an additional acceptance factor that depends on $E_{h a d}$ is applied to each event.

An $E_{h a d}$-dependent correction factor is calculated by taking the ratio of true to estimated background from the muon gun acceptance prediction. The weight for the iron of Target 2 for the $i$ th bin of $E_{h a d}$ would be

$$
w_{i}^{E_{\text {had }}}=\frac{N_{\text {true }, i}^{\text {tgt }, F e}}{N_{\text {est }, i}^{t g t, F e}},
$$

where $N_{t r u e, i}^{t g t 2, F e}$ is the number of true plastic background events and $N_{e s t, i}^{t g t 2, F e}$ is the number of plastic background events predicted by the previous muon gun step. These scale factors for the iron of Target 5 are shown in Fig. A.4. All scale factors are shown in Appendix A.4.

\subsubsection{Accuracy}

Both muon gun and the $E_{\text {had }}$-dependent corrections are applied to events in the tracker region to obtain the final prediction for the plastic background. These events are now weighted as

$$
w=\frac{f_{a c c}^{t g t 2, F e}\left(E_{\mu}, \theta_{\mu}\right)}{f_{a c c}^{f a u x 2, F e}\left(E_{\mu}, \theta_{\mu}\right)} \cdot w_{i}^{E_{h a d}},
$$




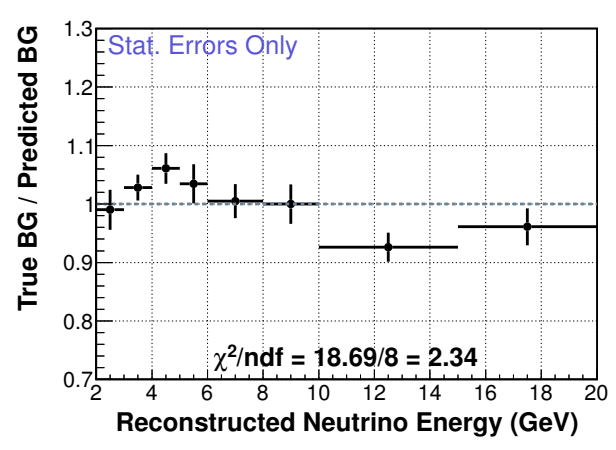

(a) $E_{\nu}$, Iron of Target 5

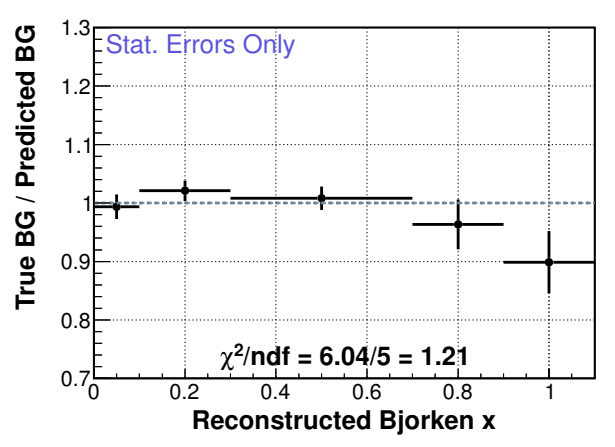

(b) $x_{b j}$, Iron of Target 5

Figure 5.10: Accuracy of the prediction of scintillator contamination in the iron of Target 5 sample as a function of $E_{\nu}$ (left) and $x_{b j}$ (right). The y axis is the ratio of the true background to the predicted background. The error bars are the statistical error of the Monte Carlo.

where all terms have been defined above.

The final procedure for estimating the distribution of background events from scintillator is as follows.

1. Events are selected in the faux targets corresponding to the passive target. Kinematic distributions are created using these events and weights from Eq. 5.4. This is done for both data and simulated samples.

2. The distribution in simulation is scaled to the true background, so the integral number of events is equal. The same scale factor will be applied to the data prediction of the background.

Figure 5.10 shows accuracy of the plastic background prediction as a function of $E_{\nu}$ and $x_{b j}$ for the iron of Target 5. Accuracy is demonstrated as a ratio of the true to predicted background. Similar plots for all targets can be found in Appendix A.4. 


\subsection{Calculating a Cross Section}

The primary results of this analysis are ratios of cross sections, but absolute cross sections are formed in the process. This section describes how those cross sections and ratios are calculated.

The total cross section for neutrino energy bin $i$ is calculated as

$$
\sigma_{i}=\frac{\sum_{j} U_{i j}\left(N_{j}-N_{j}^{b g}\right)}{\epsilon_{i} T \Phi_{i}},
$$

where $j$ is the index of a smeared $E_{\nu}$ bin, $U_{i j}$ is a function that accounts for smearing from true bin i to reconstructed bin j, $N_{j}$ is the number of selected events, $N_{j}^{b g}$ is the estimated number of background events, $\epsilon_{i}$ is the efficiency for reconstructing signal events, $T$ is the number of target nucleons, and $\Phi_{i}$ is the flux in bin $i$.

The flux-integrated differential cross section for an $x_{b j}$ bin $i$ is

$$
\left(\frac{d \sigma}{d x_{b j}}\right)_{i}=\frac{\sum_{j} U_{i j}\left(N_{j}-N_{j}^{b g}\right)}{\epsilon_{i} T \Phi \Delta_{i}\left(x_{b j}\right)}
$$

where $\Phi$ is the flux integrated over the acceptance range of $E_{\nu}=2-20 \mathrm{GeV}, \Delta_{i}\left(x_{b j}\right)$ is the width of the bin, and other terms have the same meaning as in Eq. 5.5

The event selection yielding $N_{j}$ was described in Sec. 5.1. Background estimation $N_{j}^{b g}$ was described in Sec. 5.4. Target numbers $T$ are in Table 5.2. Bin migration $U_{i j}$ is discussed in Sec.5.5.1. The efficiency correction $\epsilon_{i}$ is outlined in Sec.5.5.2. The flux is not necessary to the formation of ratios but is discussed in Sec. 5.5.3.

\subsubsection{Bin Migration and Unfolding}

Finite detector resolution and imperfect reconstruction cause the reconstructed kinematics of an event to differ from its true kinematics. This smearing can cause events to migrate from one bin of a histogram to another. The effects of detector smearing can be estimated using a full simulation of neutrino events in the detector. Several techniques exist to unfold reconstructed 
distributions using a smearing matrix, but they all introduce either a bias from the model used in the simulation or erratic statistical fluctuations. When migration is significant or the distribution is not well modeled, unfolding distributions is unwise. Examples of migration matrices are shown in Figs. 5.11. The full set of migration matrices is in Appendix A.7

The background-subtracted reconstructed $E_{\nu}$ distribution is unfolded into true kinematics $E_{\nu}$ distributions for each target section using the iterative Bayesian technique with 4 iterations [62]. The nuclear effects of the $E_{\nu}$ distribution are well simulated so any introduced model dependence is negligible.

Reconstructed $x_{b j}$ distributions are not unfolded because that may introduce a strong model dependence on $x_{b j}$-dependent nuclear effects that are not well simulated. In addition, the smearing in $x_{b j}$ is significant and asymmetric; both features can cause problems for unfolding. Models which predict distributions in generated kinematics must be folded by multiplying by the migration matrix in order to be compared to $x_{b j}$ distributions in this analysis.

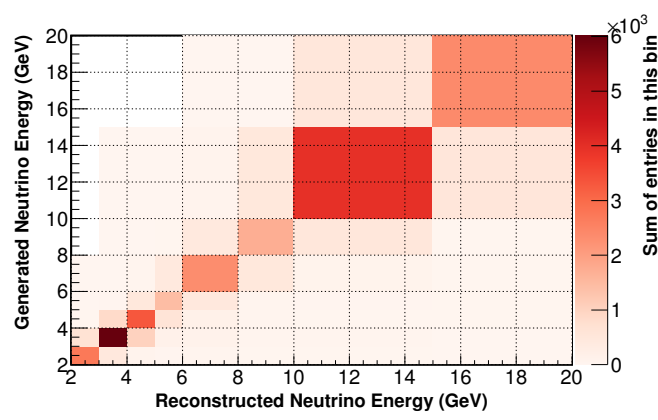

(a) $E_{\nu}$, Lead of Target 4

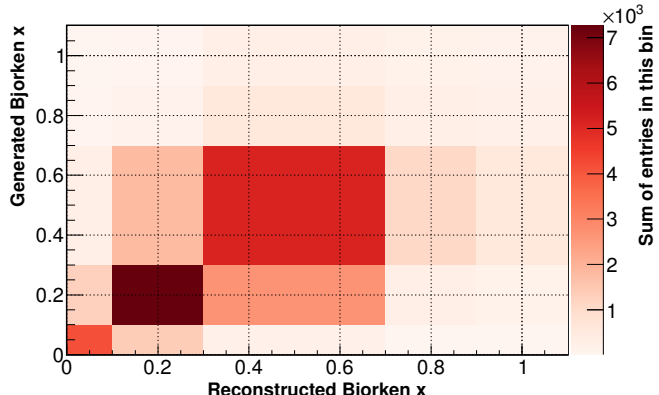

(b) $x_{b j}$, Lead of Target 4

Figure 5.11: Bin migration matrices for $E_{\nu}$ (left) and $x_{b j}$ (right) calculated with simulation. The $\mathrm{x}$-axis is the reconstructed quantity and the $\mathrm{y}$-axis is the generated quantity.

\subsubsection{Efficiency and Acceptance Corrections}

Not all generated events are observed due to limited detector acceptance and reconstruction inefficiency. The primary reason for the loss of events is the geometrical acceptance requirement that the muon is matched to a track in MINOS. The other reason for lost events is 
reconstruction failures in MINERvA or MINOS. It is preferable, but not always possible, to measure efficiency using the data to avoid entangling the measurement with the models in the simulation.

\section{Muon Tracking Efficiency}

The muon tracking efficiency has been measured in MINERvA and MINOS separately [63]. These measurements only address the efficiency of muons that propagate from MINERvA to MINOS and do not incorporate any geometric acceptance effects.

To measure the tracking efficiency in MINERvA, a sample of events was selected which have a track in MINOS that is pointing into the MINERvA fiducial area and also have muon-like activity in the MINERvA detector. The test for muon-like activity was very crude and required no tracking. The fraction of such events that also had a track in MINERvA is the tracking efficiency in MINERvA. This efficiency was measured as a function of the muon momentum reconstructed in MINOS.

To measure the tracking efficiency in MINOS, the inverse of the above study was performed. This efficiency was measured by identifying events with a track in MINERvA that point to the MINOS fiducial area. Efficiency is the fraction of such events that had a corresponding track in MINOS. It is not possible to make a momentum-dependent version of this measurement because there is no reconstructed muon momentum for events that are not reconstructed by MINOS. Instead, the sample is coarsely divided into low and high momentum at $3 \mathrm{GeV} / c$ by using the magnitude of curvature in MINERvA caused by multiple scattering. The high momentum sample contains muons that were deflected by less than $1 \mathrm{~cm}$ in the MINERvA downstream calorimeters. The low momentum sample muons were deflected by more than $4 \mathrm{~cm}$.

The tracking efficiency in both detectors, especially MINOS, is sensitive to the intensity of the neutrino spill. The higher the intensity, the more likely it is that overlapping activity will 
obscure the muon or confuse the tracking algorithms. For this reason, the tracking efficiency of both detectors was measured for every run period. These measurements were compared to simulation to derive a efficiency correction. The efficiency corrections are summarized in Table 5.4

\section{Remaining Reconstruction Efficiency}

All remaining loss of signal, including that due to geometric acceptance limitations, is calculated using the full MINERvA simulation. Efficiency for true kinematics bin $i$ is measured in simulation as the fraction of generated events which are observed and selection in the event sample

$$
\epsilon_{i}=\frac{N_{i, s i g}^{o b s .}}{N_{i, s i g .}^{g e n .}} .
$$

The numerator $N_{i, s i g}^{\text {obs. }}$ is the number of generated signal events in bin $i$ which pass the event selection fiducial requirements. The denominator $N_{i, \text { sig. }}^{g e n}$ is the number of generated signal events in bin $i$. Note that the numerator definition does not require that the generated event is a charged-current $\nu_{\mu}$ event, because the simulation will be used directly to predict the backgrounds from NC and WS events, as discussed in Sec. 5.3.1

Distributions of $x_{b j}$ have not been unfolded into true kinematics space. It is not possible to calculate the efficiency directly in reconstructed bins, because reconstructed quantities are not defined for most events that are not observed (e.g. there is no muon energy for an event that has no reconstructed muon). The efficiency measured in true kinematic space must be folded into detector-smeared space in order to apply the efficiency correction. Folding is performed by multiplying the efficiency in Eq. 5.7 by the migration matrix. The equation for the folded efficiency for reconstructed kinematics bin $\mathrm{j}$ is

$$
\epsilon_{j}=\sum_{i} U_{j i} \epsilon_{i}
$$

where $U_{j i}$ is the migration matrix, $\epsilon$ is the efficiency, and the sum is over bins $i$ in true kinematic space. 


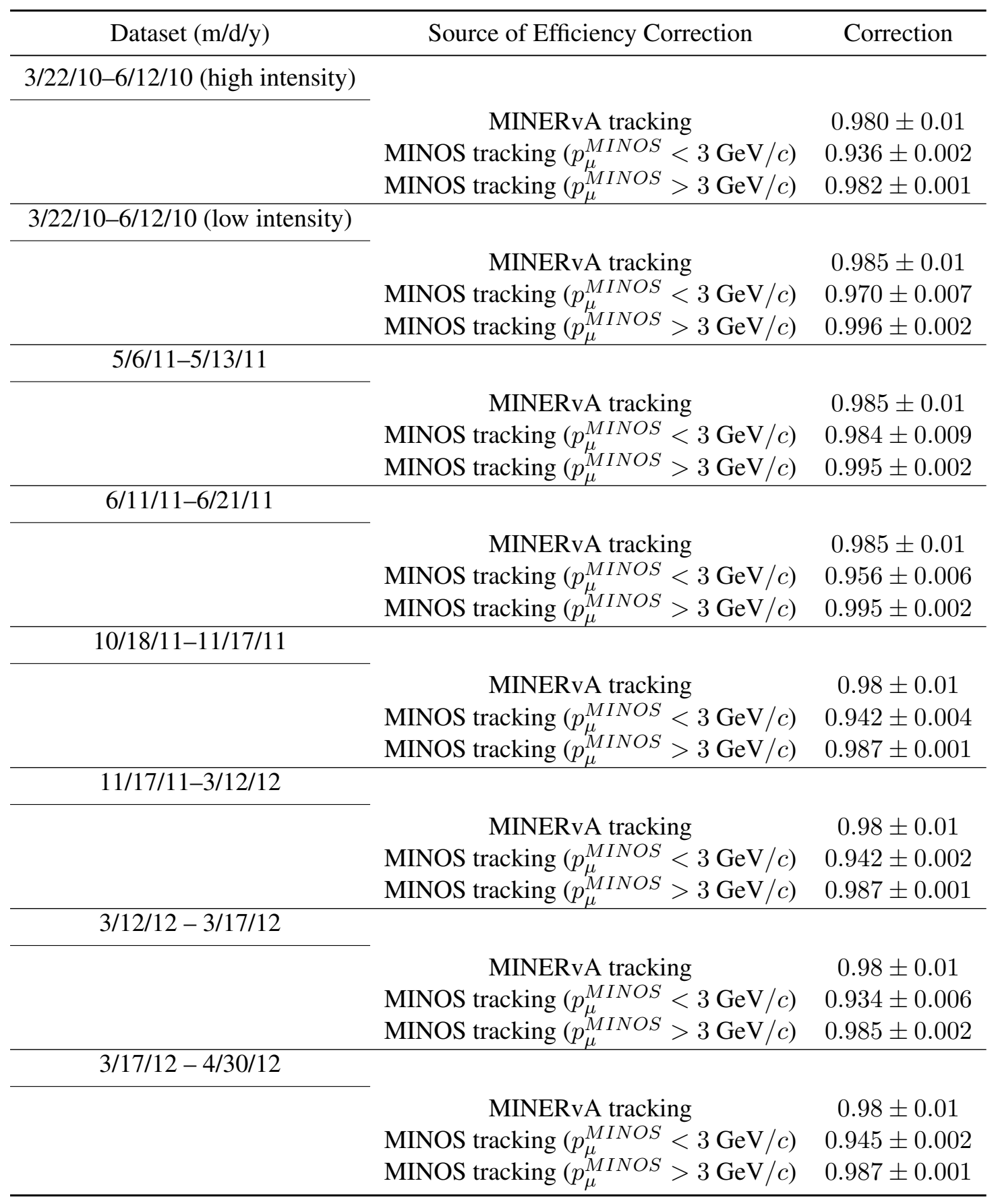

Table 5.4: Corrections to the muon reconstruction efficiency in MINERvA and MINOS. 
Figure 5.12 shows the efficiency for events from the lead of Target 4 as a function of $E_{\nu}$, $x_{b j}$, and $\theta_{\mu}$. Efficiency is defined as the fraction of signal events that pass all event selection criteria. Purity is the fraction of events in the sample that are generated signal events. The efficiency for all target samples can be found in Appendix A.6
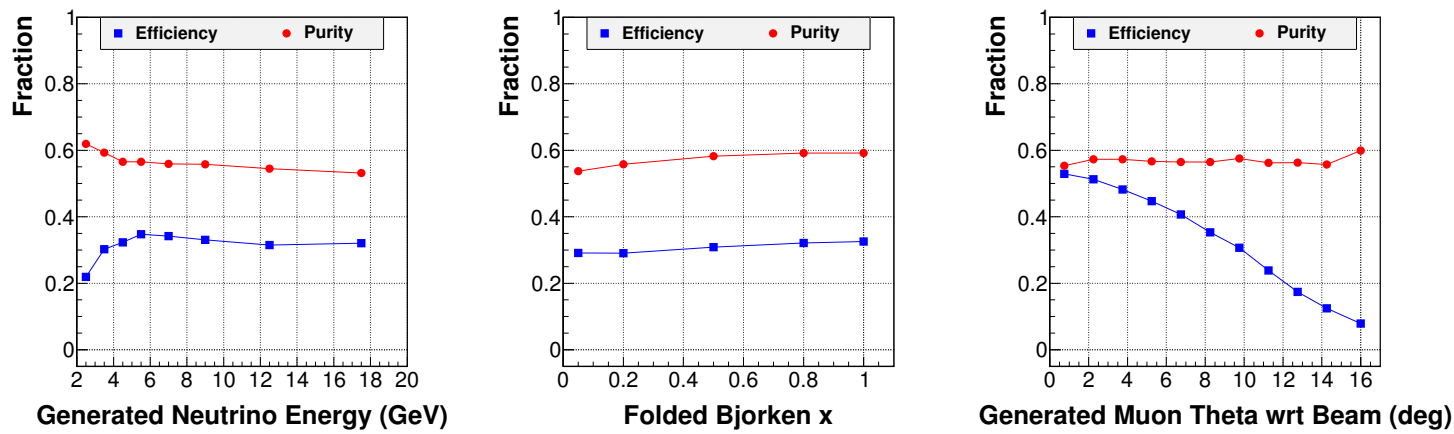

Figure 5.12: Monte Carlo calculation of efficiency (blue) and purity (blue) for the lead of Target 4 as a function of $E_{\nu}$ (left), $x_{b j}$ (center), and $\theta_{\mu}$ (right). Efficiency is defined as the fraction of signal events selected. Purity is the fraction of selected events that are signal; the major source of impurity is the scintillator background. These plots are representative of all targets.

\subsubsection{Flux}

The goal of this analysis is to form ratios of cross sections. The flux is the same throughout all targets, because the radius of the neutrino beam is wide enough that there is no variation across the detector. The flux cancels in the calculation of ratios and can simply be ignored when calculating ratios of cross sections. However, because absolute cross sections are interesting and provide an important check on the reliability of the analysis, they are included in this dissertation. These absolute cross sections should not be considered a result produced by MINERvA, as the flux contains far more uncertainty than is ultimately achievable. Absolute cross sections are formed by dividing the efficiency corrected signal by the number of incident neutrinos to form the cross section. The flux used in this analysis is shown in Table 5.5 and Fig. 5.13 


\begin{tabular}{cc}
\hline$E_{\nu}$ Bin $(\mathrm{GeV})$ & $\nu_{\mu}$ Flux \\
\hline $1.50-2.00$ & 0.310 \\
$2.00-2.50$ & 0.409 \\
$2.50-3.00$ & 0.501 \\
$3.00-3.50$ & 0.526 \\
$3.50-4.00$ & 0.419 \\
$4.00-4.50$ & 0.253 \\
$4.50-5.00$ & 0.137 \\
$5.00-5.50$ & 0.080 \\
$5.50-6.00$ & 0.055 \\
$6.00-6.50$ & 0.042 \\
$6.50-7.00$ & 0.036 \\
$7.00-7.50$ & 0.030 \\
$7.50-8.00$ & 0.027 \\
$8.00-8.50$ & 0.024 \\
$8.50-9.00$ & 0.021 \\
$9.00-9.50$ & 0.019 \\
$9.50-10.00$ & 0.017 \\
$10.00-11.00$ & 0.030 \\
$11.00-12.00$ & 0.025 \\
$12.00-13.00$ & 0.021 \\
$13.00-14.00$ & 0.018 \\
$14.00-15.00$ & 0.015 \\
$15.00-16.00$ & 0.012 \\
$16.00-17.00$ & 0.010 \\
$17.00-18.00$ & 0.009 \\
$18.00-19.00$ & 0.007 \\
$19.00-20.00$ & 0.006 \\
$20.00-21.00$ & 0.005 \\
$21.00-22.00$ & 0.004 \\
$22.00-23.00$ & 0.004 \\
$23.00-24.00$ & 0.003 \\
$24.00-25.00$ & 0.002 \\
\hline
\end{tabular}

Table 5.5: Flux of $\nu_{\mu}$ 's in the low energy forward horn current tune of the NuMI beam in units of $\times 10^{-8}$ neutrinos per $\mathrm{cm}^{2}$ per P.O.T. 


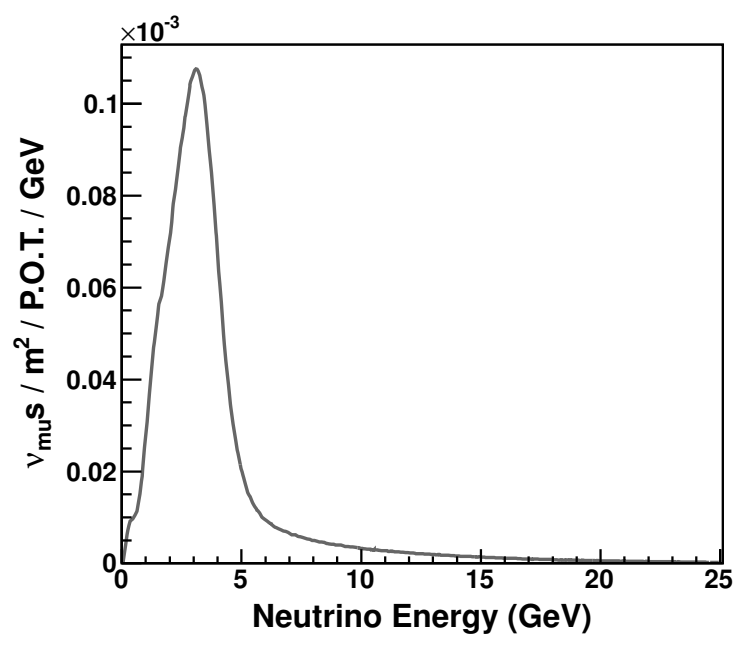

Figure 5.13: Prediction of the $\nu_{\mu}$ flux in the NuMI beam forward horn current (neutrino mode) during the Low Energy Run. Units are number of $\nu_{\mu}$ 's per incident proton per $m^{2}$ per GeV.

\subsubsection{Isoscalar Correction}

MINERvA does not currently have the ability to differentiate a neutrino interaction with a neutron from that with a proton, which would require reconstructing the charge of all final state particles. The average cross section of nucleons in a nucleus is measured instead. The neutrino-proton cross section is different from the neutrino-neutron cross section. This means that the average per-nucleon cross section of a nucleus depends on its neutron excess $\mathbb{1}^{1}$ In the absence of nuclear effects, the average cross section per nucleon of a nucleus with $Z$ protons and $N$ neutrons is

$$
\bar{\sigma}_{Z, N}=\frac{1}{Z+N}\left(Z * \sigma_{p}+N * \sigma_{n}\right)
$$

where $\sigma_{p}$ and $\sigma_{n}$ are the free nucleon cross sections for the proton and neutron respectively. Note that the average cross section per nucleon is the same for all isoscalar targets $(Z=N)$, but for non-isoscalar targets $\bar{\sigma}_{Z, N}$ differs due to neutron excess.

Deviations from the sum of free nucleon cross sections arise also due to nuclear effects,

\footnotetext{
${ }^{1}$ Neutron excess is the the ratio of the number of neutrons to protons in the nucleus. This is greater than 1 (thus "excess") for stable nuclei because Coulomb repulsion among protons makes them harder to bind.
} 

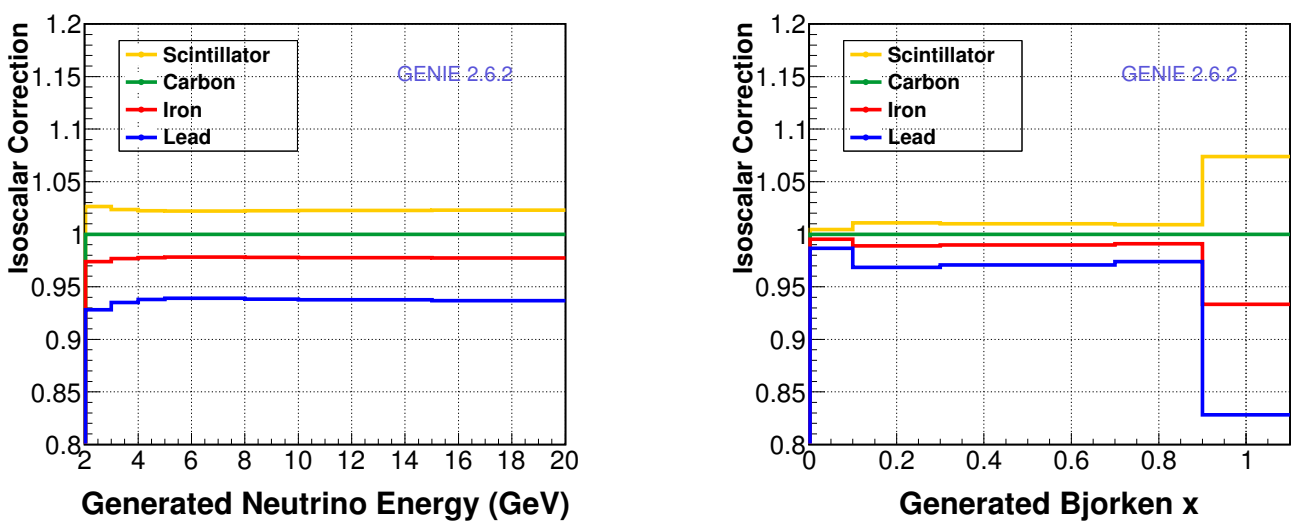

Figure 5.14: Isoscalar correction applied to the cross section of each nuclear target. The corrections are derived using predictions of free nucleon cross sections in GENIE 2.6.2 and GRV98nlo PDFs.

which this analysis measures. An isoscalar correction removes the effects of neutron excess.

The isoscalar correction is

$$
f_{Z, N}^{i s o}=\frac{\left(Z * \sigma_{p}+N * \sigma_{n}\right) /(Z+N)}{\left(\sigma_{p}+\sigma_{n}\right) / 2} .
$$

The isoscalar-corrected ratio of cross sections of nuclei A and B is then

$$
\frac{\sigma_{A}^{\prime}}{\sigma_{B}^{\prime}}=\frac{\sigma_{A}}{\sigma_{B}} * \frac{\frac{1}{Z_{B}+N_{B}}\left(Z_{B} * \sigma_{p}+N_{B} * \sigma_{n}\right)}{\frac{1}{Z_{A}+N_{A}}\left(Z_{A} * \sigma_{p}+N_{A} * \sigma_{n}\right)}=\frac{F_{A}}{F_{B}},
$$

which measures the difference in nuclear effects $\frac{F_{A}}{F_{B}}$. MINERvA does not have a hydrogen target and no experiment has a free neutron target, so GENIE's free nucleon cross sections are used. The isoscalar correction for each nucleus Fig. 5.14 and for the ratio of of passive nuclei to scintillator in Fig. 5.15 .

\subsubsection{Absolute Cross Section Results}

Measurements of absolute cross sections and their comparisons to the MINERvA Monte Carlo are shown in Appendix A.8. A representative example of these results in Fig. 5.16 shows the measurement of the inclusive cross section in scintillator and a comparison to Monte Carlo. The discrepancy between data and Monte Carlo is consistent with measurements of other interaction channels in MINERvA. The reason for the difference in the cross section as a function of 

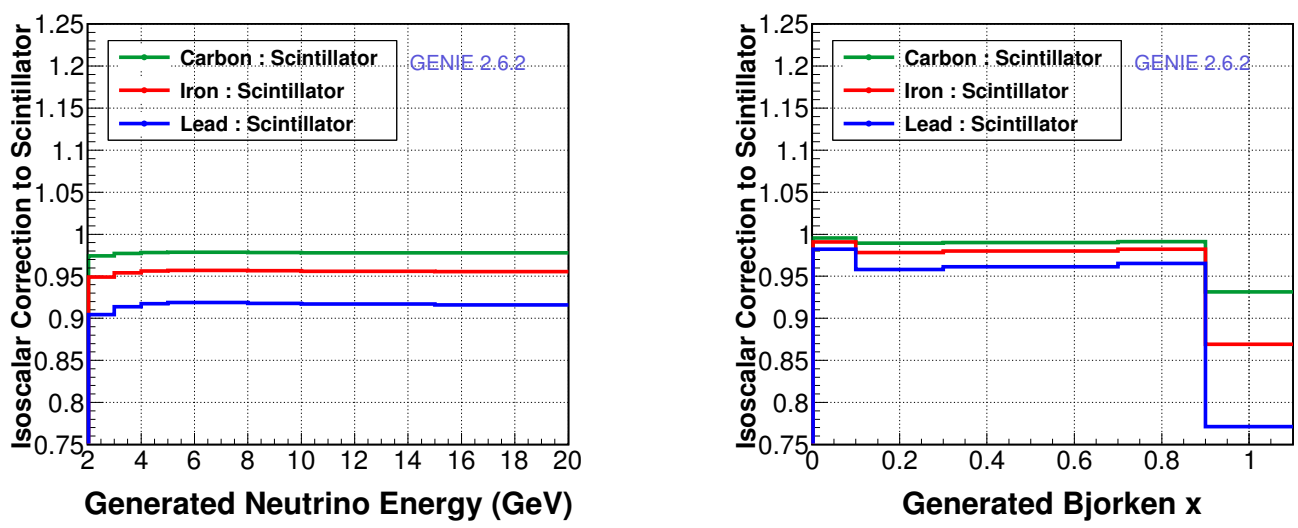

Figure 5.15: Isoscalar correction applied to the ratio of cross sections for each passive nucleus. The corrections are derived using predictions of free nucleon cross sections in GENIE 2.6.2 and GRV98nlo PDFs.
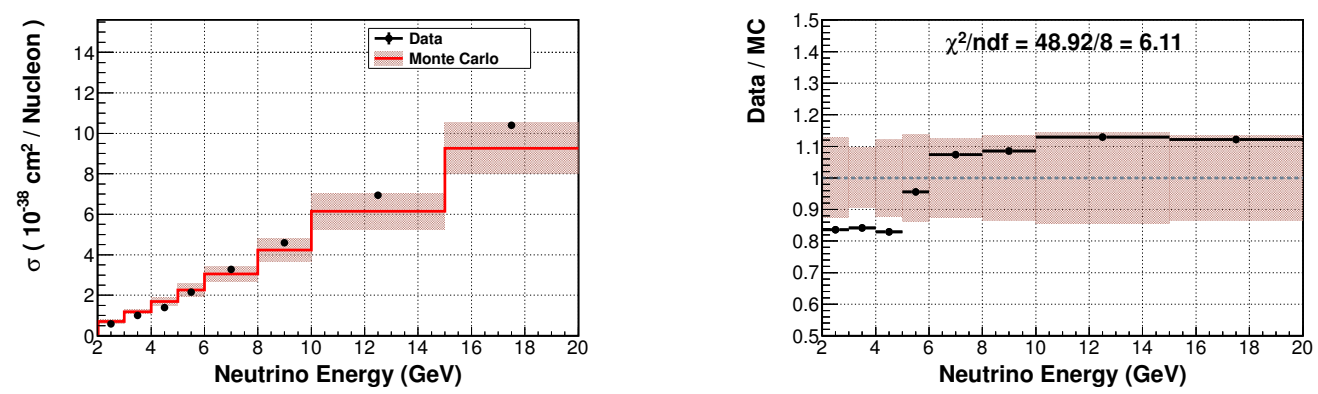

Figure 5.16: (Left) Measured and predicted absolute scintillator cross section as a function of $E_{\nu}$. (Right) Ratio of measurement to prediction.

neutrino energy is overwhelmingly due to imperfect modeling of the flux, not a difference in the neutrino interaction cross section. There are several imminent improvements to the simulation of the neutrino beam in MINERvA.

\subsubsection{Forming Ratios}

Cross section ratios are formed with the sums of efficiency-corrected distributions, taking into account the number of targets. Dividing by the flux is not necessary because the flux has no spatial dependence throughout the detector; the flux is identical through all targets. Uncertainties in the flux model are considered and contribute a small uncertainty on the ratio, 


\begin{tabular}{cc}
\hline Target Number & Scintillator Modules \\
\hline 2 & $27-32,51-56,63-68$ \\
3 & $33-38,57-62,75-80$ \\
4 & $45-50$ \\
5 & $39-44,69-74$ \\
\hline
\end{tabular}

Table 5.6: Listing of scintillator modules paired to each passive target assembly. This is done to keep the ratios statistically independent. The assignments are chosen to minimize systematic bias by varying the transverse and longitudinal areas of the modules.

which is discussed in Sec. 5.6.2. The ratio of cross sections is formed simply by dividing the cross section of one nucleus by another nucleus.

A statistically independent scintillator sample is used to form each of the ratios of carbon, iron, and lead. The tracking modules in each sample are chosen using faux targets so that the areal shape of scintillator matches that of the target sections that comprise the target nucleus. Modules that are matched to each target come from varied longitudinal position to further avoid systematic bias. The modules used as the denominator in the ratio for each target are listed in Table 5.6 .

\subsubsection{Summing Over Targets}

Event samples have thus far been separated by their target section or faux target; each has its own background, migration matrix, and efficiency correction because the reconstruction and acceptance differs across targets. This segregation increases the accuracy of all the above corrections. Event samples of similar nuclei are added after the efficiency correction is applied, since at that point all such differences have been removed. For example, the efficiencycorrected samples of the iron from Targets 2,3 , and 5 are added. The compatibility of measurements in the different targets is shown in Fig. 5.17 The figure shows cross section ratios. The cross sections of the passive carbon, iron, and lead targets are normalized by the cross section on scintillator using the pairings of Table 5.6. The isoscalar correction has been applied. These 


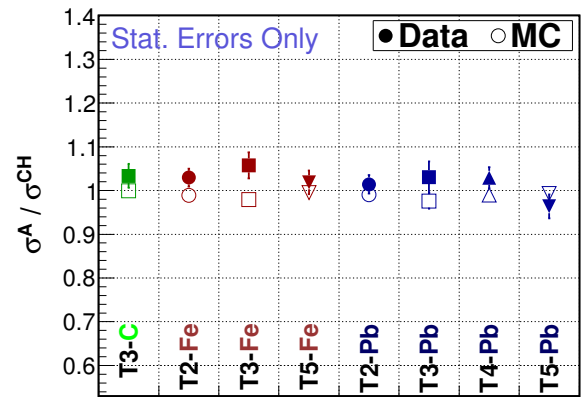

(a) $E_{\nu}=[2,20] \mathrm{GeV}$

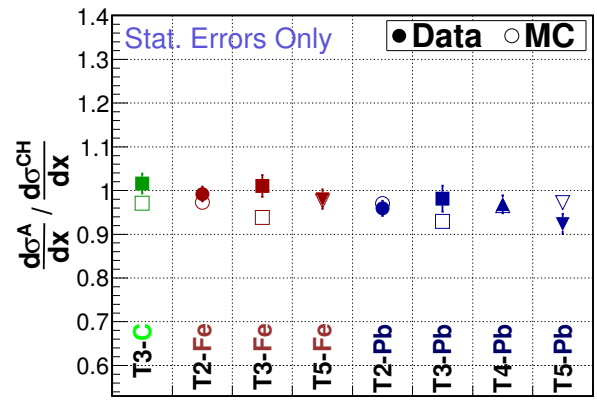

(b) $x_{b j}=[0.0,1.1]$

Figure 5.17: Cross section ratios of carbon, iron, and lead to scintillator measured with each passive target assembly. Data are shown in filled in points, Monte Carlo in the hollow points. Only statistical errors are shown.

plots suggest that the measurements of the cross sections using different targets are compatible and can thus be added without systematic bias. Similar comparisons are made for each bin of $E_{\nu}$ and $x_{b j}$; these can be found in Appendix A.9.

\subsection{Systematic Uncertainties}

\subsubsection{Evaluation of Systematic Errors}

Systematic errors are evaluated using the "many universes" procedure [64]. Each source of systematic error is represented by a deviation from the nominal values. The entire cross section calculation is repeated assuming a deviation in the nominal value. The calculation with a deviation is referred to as a "universe" because it represents a hypothetical scenario where, for example, the flux is $5 \%$ higher. Sometimes it is appropriate to use an ensemble of universes representing possible deviations from the nominal value.

The covariance matrix representing the error on the cross section as a result of a source of systematic uncertainty is derived from the spread of the cross section as measured in the many universes. There are two ways of calculating the covariance matrix: spread and maximum deviation. 


\section{Spread}

Statistical tests can be used to determine the spread among the cross section universes that result from systematic deviations. When the deviations are expected to resemble a Gaussian distribution, the error on the cross section in a bin is equal to the root mean squared (RMS) of the values of the cross section for all universes in that bin. The covariance matrix element relating bin $i$ and bin $j$ calculated with the RMS method is

$$
\operatorname{cov}_{i, j}=\frac{1}{N} \sum_{k=1}^{N}\left(x_{k i}-\bar{x}_{i}\right)\left(x_{k j}-\bar{x}_{j}\right) .
$$

When the Gaussian assumption is not appropriate, the interquartile range (IQR) of the cross section in all universes is used. The IQR is defined as the distance between the boundaries at the bottom $25 \%$ and top $25 \%$ of the distribution. For a Gaussian distribution $I Q R=.6745 \sigma$. A covariance matrix element relating bin $i$ and bin $j$ calculated with the IQR method is

$$
V_{i, j}=(0.741 * I Q R(i))(0.741 * I Q R(j))
$$

\section{Maximum Deviation}

The maximum deviation among the universes is another measure of an error. This method is used when there are too few universes to use a more sophisticated statistical test of the spread among the universes. Any systematic error evaluated with less than ten universes uses the deviation method. If there is only one deviation from the nominal value (one universe), the distance between the deviation and nominal value is the error on the cross section. If there are more than two deviations, then half of the size of the largest difference in deviations is used. A covariance matrix element relating bin $i$ and bin $j$ calculated with the maximum deviation method is

$$
V_{i, j}=\Delta_{\max }(i) \Delta_{\max }(j)
$$




\section{Procedure for Systematics on Measurable Quantities}

Systematics on measurable quantities (i.e. muon energy, muon angle, recoil energy) produce lateral shifts. If the observed value for one of these quantities differs from the true value, the event can be added to the wrong bin in a histogram, changing the distribution. Such uncertainties are assessed under the assumption that systematic deviations can be represented as a Gaussian distribution with some standard deviation $\sigma$. First, $\sigma$ is determined as a systematic difference between data and Monte Carlo in the reconstruction of the quantity. Next, an ensemble of 60 random shift factors $s_{i}$ are pulled from a Gaussian which is centered on 0 with its $\sigma$ determined in the previous step. These random factors represent the deviation from the nominal value; the analysis is repeated in each of these universes. The shifted value of a measured quantity in one universe may cross the bounds of the signal region. In this case, the event is rejected in only that universe. The cross section is calculated in all 60 universes, and the error on the cross section measurement comes from the RMS spread method.

\subsubsection{Flux}

Flux is a notorious uncertainty in most neutrino experiments. The fundamental difficulty in knowing the neutrino flux is that the final beamline products, the neutrinos, cannot be easily measured except by the very interactions which are being studied by the experiment. This is why many neutrino analyses, including this analysis, are designed to mitigate dependence on the simulation of the flux. Error on the flux comes from uncertainty in the cross sections of hadron-producing $p C$ interactions and the focusing of these hadrons in the beamline.

The dominant source of uncertainty on the flux from the NuMI beam comes from the primary $p \mathbf{C}$ interactions. The cross section for a $p \mathbf{C}$ interaction to produce a final state particle that will produce a neutrino through meson decay is not well simulated for interactions that take place at $120 \mathrm{GeV} / c$, the momentum of incident protons in NuMI. Hadron production

cross section measurements of $p C \rightarrow \pi^{ \pm}$and $p C \rightarrow K^{ \pm}$interactions are used to constrain 


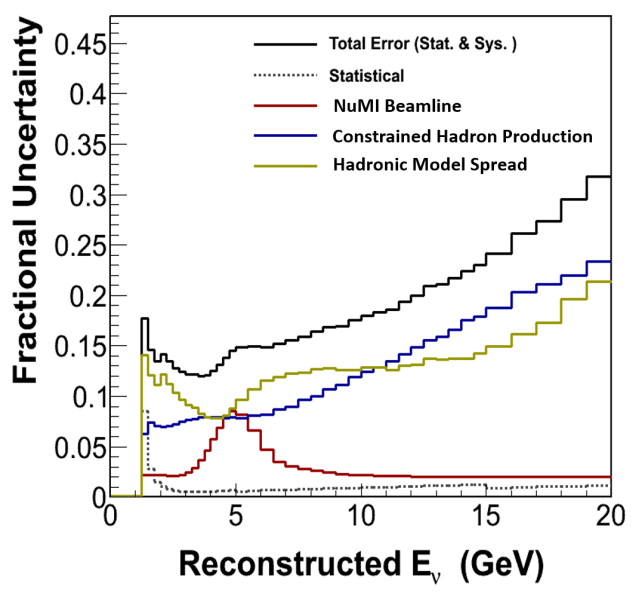

Figure 5.18: Flux errors on the charged current $\nu_{\mu}$ event rate. The three uncorrelated sources of systematic uncertainty are discussed in detail in Sec. 5.6.2

the simulation where possible, which greatly reduces the uncertainty. Other uncertainties are related to the beamline apparatus.

These sources of uncertainty are uncorrelated. Figure 5.18 shows a summary of the flux errors on the reconstructed neutrino energy spectrum for charged current events.

\section{Constrained Hadron Production}

When a simulated $p \mathrm{C}$ interaction is constrained by external data, the uncertainty on that interaction is the uncertainty on the constraining cross section measurement.

There is no available analysis of data on a NuMI target at $120 \mathrm{GeV} / c$, so available data must be interpolated ${ }^{2}$ The primary dataset that constrains hadron production in the NuMI beam is NA49, which measured $p \mathrm{C}$ interactions at $158 \mathrm{GeV} / c$ [44]. These data are related to NuMI energies by Feynman scaling. The uncertainty in this scaling procedure was evaluated by scaling from from NA49 momenta $(158 \mathrm{GeV} / c)$ to NA61 momenta $(31 \mathrm{GeV} / c)$ [66]. The differences between NA61 data and scaled NA49 data were found to have a negligible impact on the neutrino flux [67].

\footnotetext{
2 The MIPP collaboration recorded $p \mathrm{C}$ collisions on a NuMI target at $120 \mathrm{GeV} / c$, but it has not yet been analyzed [65].
} 


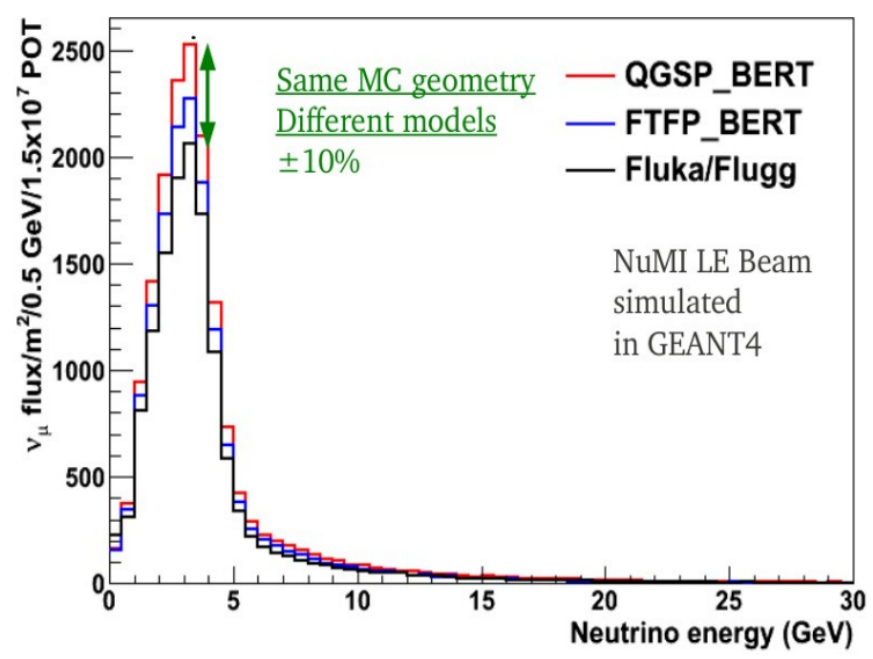

Figure 5.19: Predictions for the NuMI neutrino flux using three different hadronic models: Geant4's implementation of QGSP_BERT and FTFP_BERT, and Fluka. The maximum spread among the models is the error on the flux for hadronic interactions that are not constrained by hadronic production cross section data. Reprinted from [34], Copyright (2011) with permission from M. Kordosky.

\section{Hadronic Model Spread}

The error on the cross section of $p \mathrm{C}$ interactions that are not constrained by data is evaluated using comparisons among simulation models. Three different hadronic models are used to simulate $p$ C interactions to predict the neutrino flux, as shown in Fig. 5.19. The models were the Geant4 implementation of QGSP_BERT and FTFP_BERT [68], and Fluka [46]. The maximum spread among these models is the error on the flux contributed by $p \mathrm{C}$ interactions that are not constrained by external data.

\section{NuMI Beamline}

Errors on the flux due to uncertainties in the NuMI beamline were evaluated for the MINOS experiment [69]. Reasonable uncertainties on NuMI beamline components were evaluated by considering performance of beamline monitoring equipment and the precision in construction of beamline components. Table 5.7 shows the uncertainties in the NuMI beamline. These 


\begin{tabular}{cc}
\hline Source & Uncertainty \\
\hline Number of protons on target & $2.0 \%$ \\
Horn transverse misalignment & $1.0 \mathrm{~mm}$ \\
Horn tilt & $0.2 \mathrm{mrad}$ \\
Horn current miscalibration & $1.0 \%$ \\
Horn current distribution & $\delta=6 \mathrm{~mm} / \delta=\infty$ \\
Baffle scraping & $0.25 \%$ \\
Misalignment of shielding blocks & $1.0 \mathrm{~cm}$ \\
Target density & $2.0 \%$ \\
\hline
\end{tabular}

Table 5.7: Uncertainties on NuMI beamline components. Reprinted from [69], Copyright (2008) with permission from $\check{Z}$. Pavlović.

uncertainties were propagated to the flux prediction using Fluka [46] to simulate hadron production in the NuMI target. Figure 5.20 shows errors on the flux from beamline components. The uncertainties are largest around $5 \mathrm{GeV}$, which is the falling edge of the focusing peak.

\subsubsection{Muon Energy}

There are three contributions to the error on the muon energy reconstruction: MINOS range measurement, MINOS curvature measurement, and energy loss in MINERvA. These errors are independent and added in quadrature. The contributions to the error on the muon momentum are summarized in Table 5.8

\section{MINOS Range Measurement}

MINOS has measured the error on reconstruction of the muon momentum by range to be $2 \%$ [27]. This error arises due to uncertainty in the detector mass, simplifications in the reconstruction's implementation of the geometry, and uncertainty in the $d E / d x$ model. The MINOS range measurement error is applicable to all muons that are reconstructed in MINOS, which is all events used in this analysis. 


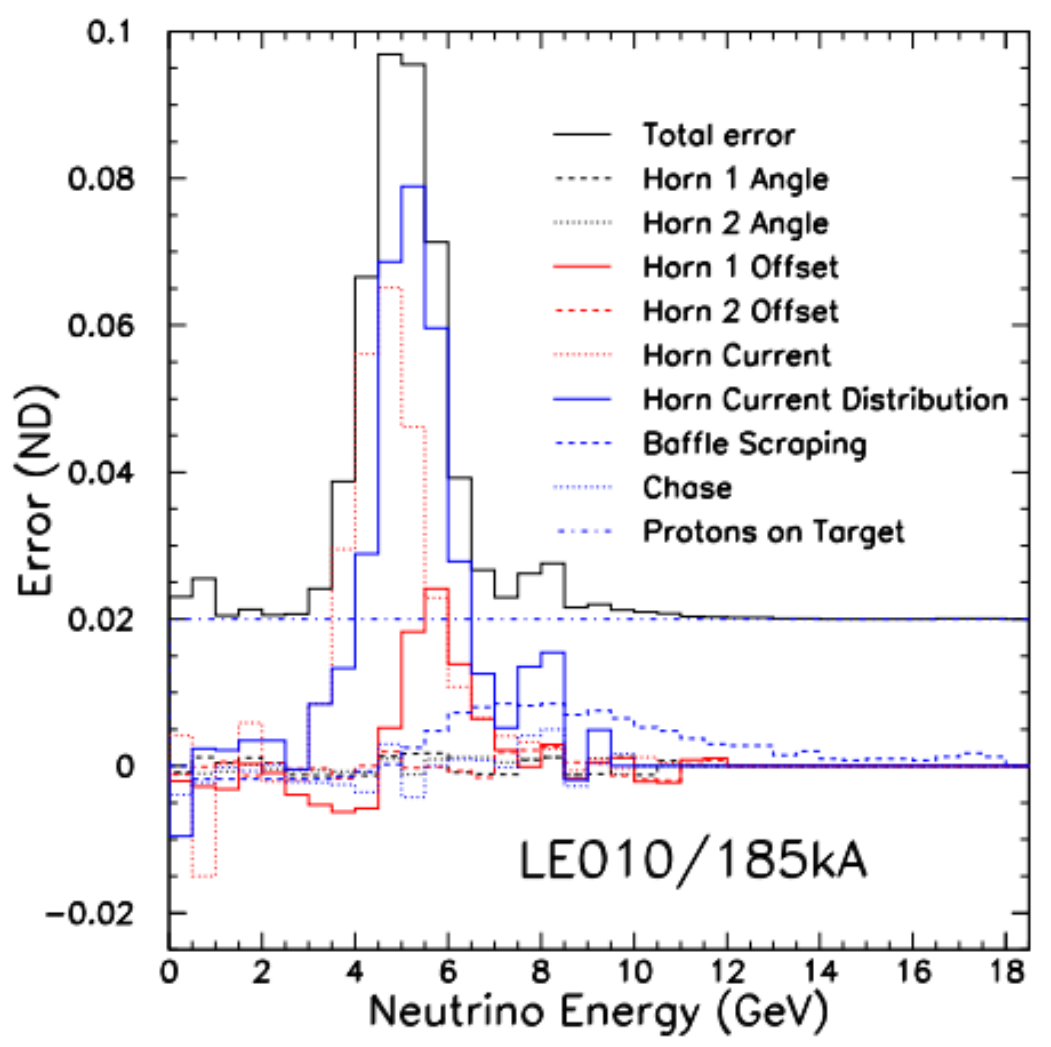

Figure 5.20: Relative errors on the flux due to uncertainties in the NuMI beamline. Reprinted from [69], Copyright (2008) with permission from $\check{Z}$. Pavlović.

\begin{tabular}{cc}
\hline Error Source & Error \\
\hline MINOS Range & $2.0 \%$ \\
\hline MINOS Curvature $\left(p_{\mu}<1 \mathrm{GeV} / c\right)$ & $2.5 \%$ \\
MINOS Curvature $\left(p_{\mu}>1 \mathrm{GeV} / c\right)$ & $0.6 \%$ \\
\hline MINERvA $d E / d x($ scintillator events) & $30 \mathrm{MeV}$ \\
MINERvA $d E / d x(\mathrm{C}, \mathrm{Fe}, \mathrm{Pb}$ events) & $40 \mathrm{MeV}$ \\
\hline MINERvA mass (scintillator events) & $11 \mathrm{MeV}$ \\
MINERvA mass $(\mathrm{C}, \mathrm{Fe}, \mathrm{Pb}$ events) & $17 \mathrm{MeV}$ \\
\hline
\end{tabular}

Table 5.8: Uncertainties on muon momentum reconstruction. The types of uncertainty are independent and added in quadrature. The MINOS range uncertainty is relevant for all muons. The MINOS curvature uncertainty is only relevant if the muon momentum was reconstructed by curvature. 


\section{MINOS Curvature Measurement}

The additional error on muon momentum when it is reconstructed by curvature in MINOS is $2.5 \%(0.6 \%)$ for a $\mu$ with less (more) than $1 \mathrm{GeV} / c[26]$. This uncertainty was measured by comparing the residual on the inverse muon momentum in data and simulation. The residual is defined as the difference in inverse muon momentum found using range and curvature:

$$
\Delta_{K}=\frac{1}{p_{\text {curv }}}-\frac{1}{p_{\text {range }}}
$$

This distribution is shown in Fig. 5.21. The residual is fit to a Gaussian. Error on reconstruction by curvature relative to range is taken to be the difference between the mean of the Gaussian fits in data and Monte Carlo, $\mu_{\text {data }}-\mu_{M C}$.

This method of evaluation uses the MINOS range reconstruction to form the residual, so the range error is added to the error on curvature for the total contribution to muon energy error budget from reconstruction in MINOS. The total error on muon momenta reconstructed by curvature is $2.1 \%(3.1 \%$ ) for momenta more (less) than $1 \mathrm{GeV} / c$.

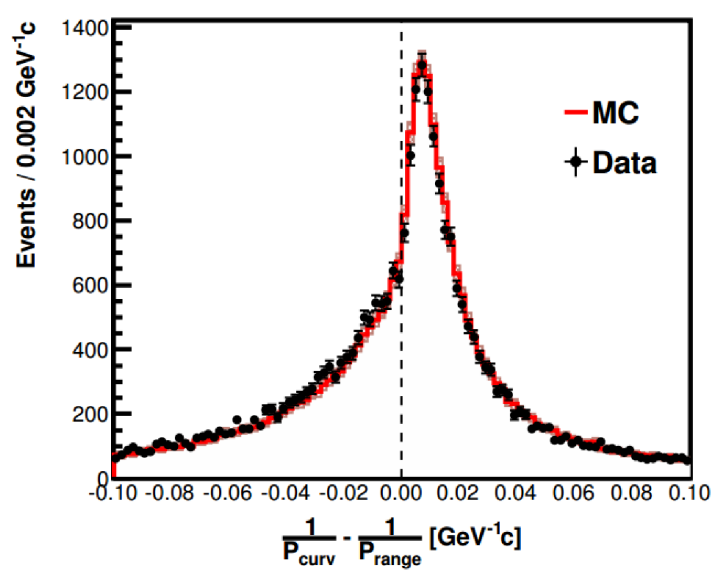

Figure 5.21: Residual in the MINOS reconstruction of the inverse muon momentum from Eq. 5.15. The difference between the data and Monte Carlo distributions is used to establish an error for the reconstruction of muon momentum by curvature relative to range. The distributions are offset from 0 by the amount of energy a muon loses in half of a MINOS plane, because the MINOS range reconstruction assumes the muon originated in the center of the most upstream MINOS plane; all of the muons for this study came from MINERvA and thus passed through the entire first plane. Figure from Ref. [26]. 


\section{Energy Loss in MINERvA}

There is additional error in energy loss in MINERvA due to uncertainties in the material assay and the $d E / d x$ model, which are independent. These errors depend on how much material the muon traverses in MINERvA, so there is a larger error assigned to events in the upstream passive nuclear targets region. The $d E / d x$ model uncertainty adds 30 (40) $\mathrm{MeV}$ to the error budget on muon energy for events in scintillator (carbon, iron, or lead). The MINERvA material mass uncertainty adds 11 (17) $\mathrm{MeV}$ to the error budget on muon energy for events in scintillator (carbon, iron, or lead).

\subsubsection{Muon Vertex and Angle Reconstruction}

Rock muon tracks are used to study the performance of track fitting. Only rock muons that enter the front of MINERvA, are matched to a track in MINOS, and have an energy less than $20 \mathrm{GeV}$ are used. These are long reliable tracks. The tracks are broken in half to create two tracks, one upstream and one downstream. The two broken tracks share the hits at the break point. Each of the broken tracks is fit independently, which yields two fitted values for the transverse position and slope of the track at the breakpoint. Differences in the values obtained from the broken tracks are fit to a double Gaussian. The position residuals are shown in Fig. 5.22, and the angle residuals are shown in Fig. 5.23, These resolutions are compared to simulation to establish the systematic error. The error on the $\mathrm{x}(\mathrm{y})$ position of the muon vertex is $0.91 \mathrm{~mm}(1.25 \mathrm{~mm})$. The error on both slopes $\left(\frac{d x}{d z}\right.$ and $\left.\frac{d x}{d z}\right)$ of the muon track is $1 \mathrm{mrad}$.

\subsubsection{Recoil Energy Reconstruction}

There are two avenues for uncertainty to enter in the reconstruction of recoil energy. The detector's response to hadronic particles is not perfectly known. The mass and calibration of the detector, energy loss models, and reconstruction methods all affect the observed energy of a particle. Recoil energy is reconstructed using a prediction from the simulation for the amount 


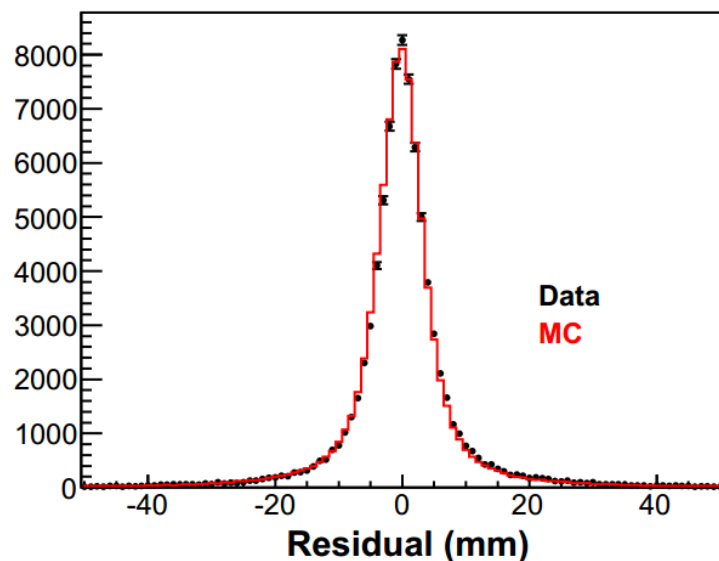

(a) x Position Residual

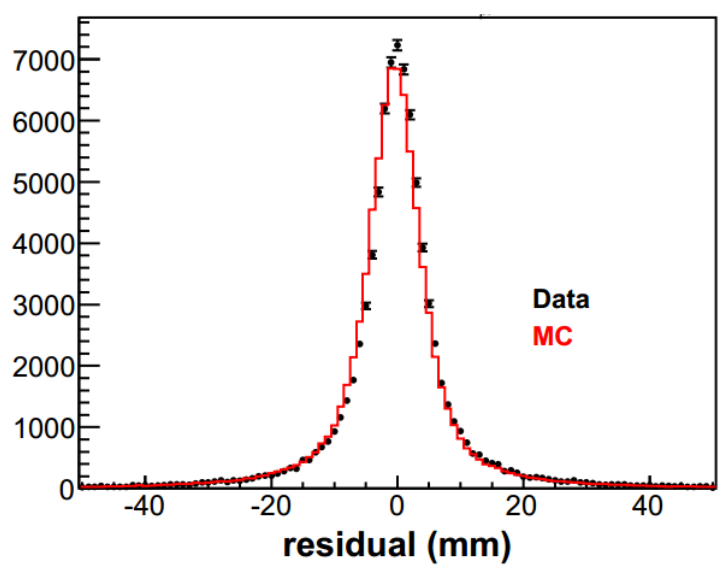

(b) y Position Residual

Figure 5.22: Residual in the position of the muon vertex. The level of agreement between data and simulation is used to establish an error in the $\mathrm{x}(\mathrm{y})$ position of $0.91 \mathrm{~mm}(1.25) \mathrm{mm}$.

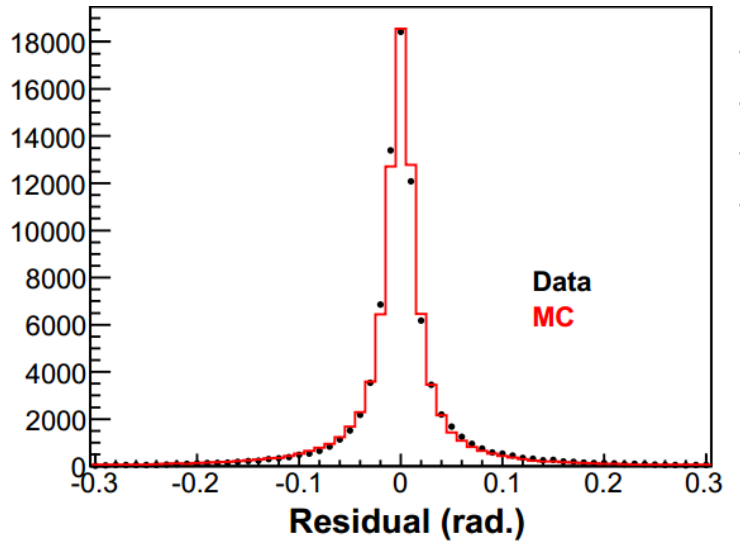

(a) $\frac{d x}{d z}$ Slope Residual

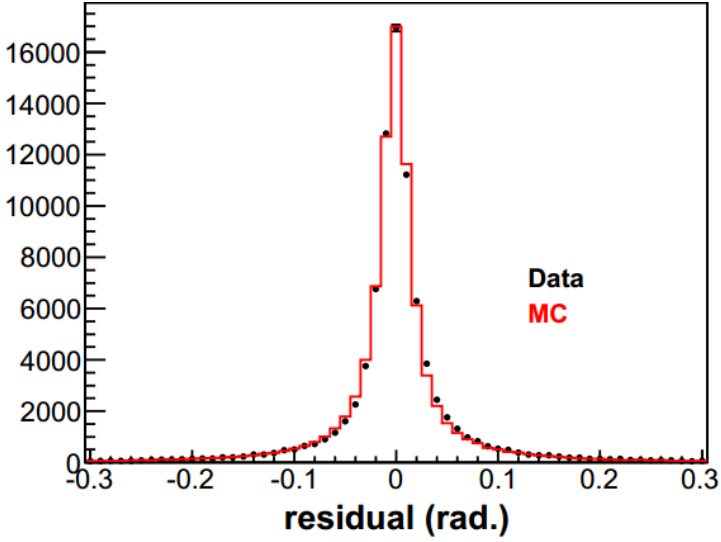

(b) $\frac{d y}{d z}$ Slope Residual

Figure 5.23: Residual in the slopes of the muon track. The level of agreement between data and simulation is used to establish an error in the muon angle of $1 \mathrm{mrad}$. Figure from Ref. [26].

of hadronic energy that is not detected. Final state interactions (FSI), or intra-nuclear scattering of final state particles, is a significant cause of hadronic energy failing to be observed. The methods for evaluating errors due to detector response and FSI are described below.

\section{Final State Interaction Models}

The $\alpha$ scale and the polyline corrections to the recoil energy of Eq. 3.3 have been tuned with a Monte Carlo sample. The FSI models used in this Monte Carlo determine what happens to a 


\begin{tabular}{cc}
\hline Model Parameter & $\delta(P) / P$ \\
\hline Pion mean free path & $\pm 20 \%$ \\
Pion charge exchange probability & $\pm 50 \%$ \\
Pion elastic reaction probability & $\pm 10 \%$ \\
Pion inelastic reaction probability & $\pm 40 \%$ \\
Pion absorption probability & $\pm 20 \%$ \\
Pion $\pi$-production probability & $\pm 20 \%$ \\
Nucleon mean free path & $\pm 20 \%$ \\
Nucleon charge exchange probability & $\pm 50 \%$ \\
Nucleon elastic reaction probability & $\pm 30 \%$ \\
Nucleon inelastic reaction probability & $\pm 40 \%$ \\
Nucleon absorption probability & $\pm 20 \%$ \\
Nucleon $\pi$-production probability & $\pm 20 \%$ \\
AGKY model $x_{f}$ distribution & $\pm 20 \%$ \\
Pion angular distribution & isotropic $\leftrightarrow$ Rein-Sehgal \\
Resonance branching ratio decay to photon & $\pm 50 \%$ \\
\hline
\end{tabular}

Table 5.9: Uncertainties on final state interaction models in GENIE. This table is based on a similar table found in [24].

particle generated by the neutrino interaction before it exits the nuclear environment. Many of these uncertainties are evaluated by reweighting Monte Carlo events. Reweightable FSI model parameters are shifted according to their uncertainty and the probability of the FSI scattering processes is recalculated. The cross section ratios analysis is repeated for each $\pm 1 \sigma$ excursion of model parameters. Half of the maximum deviation among the excursions and central value is the error from the model parameter. Model parameters are varied independently, and the resulting errors are added in quadrature. Table 5.9 lists the reweightable model parameters and their uncertainties. FSI model parameters that cannot be handled through event reweighting are evaluated with statistically independent Monte Carlo samples, which are discussed in Sec. 5.6.5

\section{Shifted Monte Carlo Samples}

Some parameters in the Monte Carlo are not reweightable. Shifted Monte Carlo samples are generated for each such parameter to assess the impact of uncertainty in the model. Half 
of the deviation of the reconstructed $E_{h a d}$ distribution for the selected events from each shifted sample from the central value is the systematic error due to uncertainty in that parameter. The parameters are uncorrelated. The procedure follows a similar study performed for the MINOS collaboration [70]. The statistics of the shifted samples are not large enough to assess a systematic for each nuclear target individually, so events are grouped into only two samples: passive targets and scintillator.

\section{Effective Nuclear Radius}

The effective nuclear radius is the size of the nucleus for low energy hadrons. It affects the overall scattering amplitude. A larger effective nuclear radius makes it more likely that the final state particle with undergo an intranuclear interaction. The effective nuclear radius is varied by an amount determined by comparisons of the FSI model with measurements of the pion-iron cross section from neutrino data. This is accomplished by shifting the FSI parameters for pion (INUKE-DelRPion) and nucleon (INUKE-DelRNucleon) in GENIE by $\pm 0.6 \mathrm{fm}$ from their respective nominal values of $0.5 \mathrm{fm}$ and $0.9 \mathrm{fm}$. Negative values for these parameters are not physical, so $0 \mathrm{fm}$ is used when the excursion would put the value below $0 \mathrm{fm}$. The errors from the positive excursion are $2 \%(0.25 \%)$ for events with $E_{\text {had }}$ less (greater) than $2 \mathrm{GeV}$. The errors from the negative excursion are $1 \%(0.25 \%)$ for events with $E_{h a d}$ less (greater) than $2 \mathrm{GeV}$

\section{Formation Time}

The formation time is a parameter in the hadronization model. It is the mean time it takes for a quark to undergo hadronization. An increase in the formation time will result in fewer intranuclear interactions and thus increase in the amount of visible energy in the detector. The nominal value of the formation time parameter (FZONE-ct0) in GENIE is $0.342 \mathrm{fm} / \mathrm{c}$, and the shifts used are $\pm 50 \%$. The excursions resulted in symmetric errors. For passive target events, 
the error is $2 \%(1 \%)$ for events with $E_{\text {had }}$ less (greater) than $2 \mathrm{GeV}$. For scintillator events, the error is $1.5 \%(0 \%)$ for events with $E_{\text {had }}$ less (greater) than $2 \mathrm{GeV}$.

\section{Hadronization Model}

The AKGY model uses a phase space reweighting scheme to simulate hadronization of final state particles. This reweighting suppresses the transverse momentum of decayed mesons with increasing $p_{t}^{2}$, which is the expected behavior. Errors on the hadronization model are evaluated by turning off the reweighting mechanism to produce a shifted sample [71]. This is accomplished in GENIE by setting the parameters KNO-PhaseSpDec-Reweight and KNOUseBaryonPdfs-xFpT2 to false. For passive target events, the error is $5 \%, 1 \%$, and $2.5 \%$ for events with $E_{\text {had }}<1 \mathrm{GeV}, 1 \mathrm{GeV}<E_{\text {had }}<10 \mathrm{GeV}$, and $10 \mathrm{GeV}<E_{\text {had }}$ respectively. For scintillator events, the error is $1 \%, 1 \%$, and $1.5 \%$ for the same energy ranges.

\section{Birks' Parameter}

Birks' empirical law for the expected light yield for energy deposited in scintillator is scaled by $\left(1+k_{B} d E / d x\right)^{-1}$, where $k_{B}$ is Birks' parameter, due to saturation effects in the scintillator. A sample of protons that stopped in the test beam detector was used to study this saturation behavior. The study found an uncertainty of $30 \%$ on the nominal value of $k_{B}=0.133 \mathrm{~mm} / \mathrm{MeV}$ used in the simulation [26]. The test beam data strongly favor a shift towards lower $k_{B}$, so the errors for this analysis are evaluated as half the difference between the nominal and $-30 \%$ excursion. The error on the charged current $\nu_{\mu}$ sample is 5\% (1\%) for events with $E_{\text {had }}$ less (greater) than $1 \mathrm{GeV}$.

\section{Detector Response}

A Monte Carlo prediction of the composition of the hits identified as the recoil system was used to evaluate the error in the detector response. The composition breaks down the 


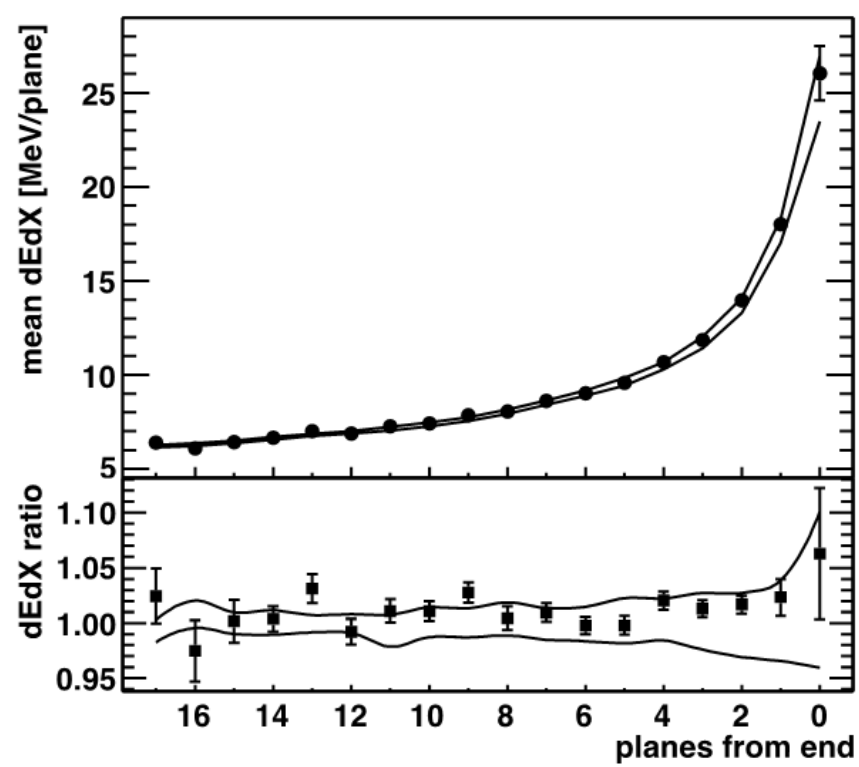

Figure 5.24: Data points show the energy loss profile of stopped protons with statistical error. The lower (upper) curves are simulations of these data with Birks' parameter shifted up (down) by $30 \%$ from the nominal value of $k_{B}=0.133 \mathrm{~mm} / \mathrm{MeV}$.

visible energy, corrected for traversed passive material, into the sources of visible energy. This procedure used only reconstructed information to identify hits, but uses generator information to identify the source responsible for the energy recorded. Examples of the composition of the recoil system are shown in Fig. 5.25. The uncertainties in the detector's response to each source of visible energy are summarized in Table 5.10 .

\section{Protons}

The proton uncertainty comes from a test beam measurement of proton kinetic energy reconstruction [26, 72]. The Fermilab Meson Test Facility beamline enables precise reconstruction of the momentum of charged particles by curvature through the use of bending magnets [73]. Protons that stop in the test beam detector were used to compare kinetic energy reconstructed by range to the beamline measurement. The ratio of measured to simulated beamline kinetic energy was histogrammed for protons stopping in each module, as shown in Fig. 5.26. The deviation of this ratio from one gives an uncertainty of $3.5 \%$ on the detector's response to 


\begin{tabular}{cc}
\hline Energy Source & Uncertainty \\
\hline Proton & $3.5 \%$ \\
Neutron $(\mathrm{KE}<50 \mathrm{MeV})$ & $25 \%$ \\
Neutron $(50<\mathrm{KE}<150 \mathrm{MeV})$ & $10 \%$ \\
Neutron $(\mathrm{KE}>150 \mathrm{MeV})$ & $20 \%$ \\
Muon & $2.4 \%$ \\
$\gamma, \pi^{0}, e^{ \pm}$ & $3 \%$ \\
$\pi^{ \pm}$, Kaon & $5 \%$ \\
Cross talk & $20 \%$ \\
Other & $20 \%$ \\
\hline
\end{tabular}

Table 5.10: Uncertainties on the detector response to different sources of light.

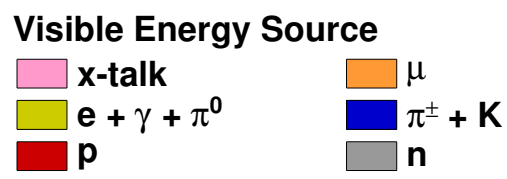

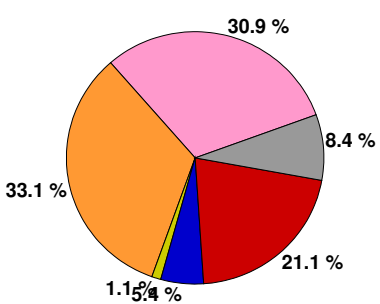

(a) $E_{\text {had }}=[0.0,0.2] \mathrm{GeV}$

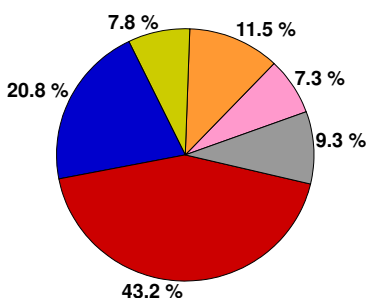

(b) $E_{\text {had }}=[0.2,1.0] \mathrm{GeV}$

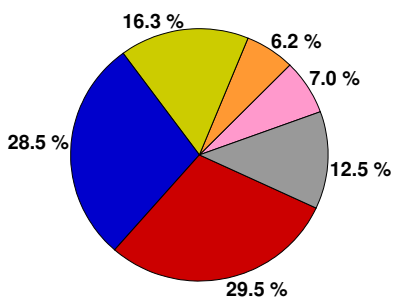

(c) $E_{\text {had }}=[1.0,2.5] \mathrm{GeV}$

Figure 5.25: Sources of visible energy in hits of the recoil system weighted by visible energy for several hadronic energy bins. These plots were made using simulation for the lead of Target 4 and are representative of events on all nuclei.

protons.

\section{Neutrons}

The neutron uncertainties come from comparisons of the $n A \rightarrow p X$ cross section in data and simulation [74]. Data for the comparisons is from [75, 76, 77, 78]. The energy dependence of the discrepancy is shown in Fig. 5.27. These uncertainties are grouped into three regions of kinetic energy and taken to be half the data-simulation discrepancy: 


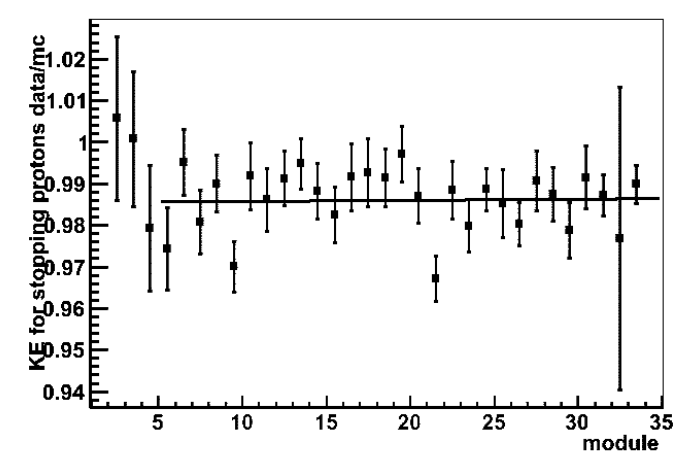

Figure 5.26: Discrepancy between data and simulation in the comparison of beamline kinetic energy to range of protons in the MINERvA test beam as a function of the module number in which the proton stops. This discrepancy is used to establish a 3.5\% error on the detector's response to protons.

- If the kinematic energy is less than $50 \mathrm{MeV}$, the error is $25 \%$.

- If the kinematic energy is between 50 and $150 \mathrm{MeV}$, the error is $10 \%$.

- If the kinematic energy is greater than $150 \mathrm{MeV}$, the error is $20 \%$.

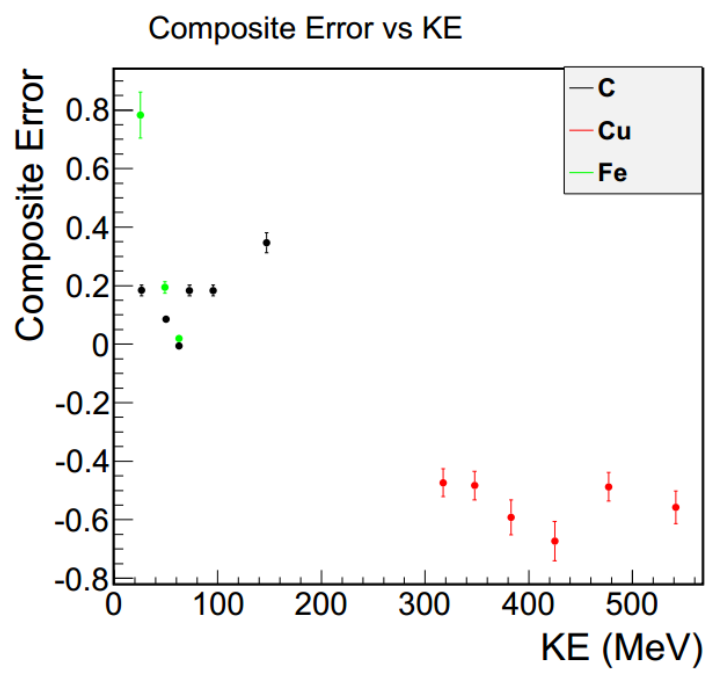

Figure 5.27: Discrepancy between data and simulation in the $n A \rightarrow p X$ cross section as a function of neutron kinematic energy (KE). The error on detector response to neutrons is half this discrepancy. It is $25 \%$ for energies less than $50 \mathrm{MeV}, 10 \%$ between 50 and $150 \mathrm{MeV}, 20 \%$ greater than $150 \mathrm{MeV}$. Figure from Ref. [74]. 


\section{Muons}

The peak of distributions of the energy deposited per plane by muons is compared to simulation to tune the absolute energy scale of the detector, as described in Sec. 3.1.2. This determination of the minimum-ionizing energy unit (MEU) depends on the accuracy of the simulation. The uncertainty on the detector mass model gives a $2 \%$ error to determination of MEU. There is a $1 \%$ uncertainty on the Bethe-Bloche process that is used to simulate the energy deposited by a muon. These two uncertainties added in quadrature yield an error on the muon response of $2.4 \%$.

\section{Electromagnetic Showers}

The detector's response to electromagnetic showers was studied using Michel electrons [26]. Michel electrons are a product of the decay of an antimuon $\left(\mu^{+} \rightarrow \bar{\nu}_{\mu}+\nu_{e}+\mathrm{e}^{+}\right)$. They appear in the detector where antimuons stop or as a tertiary product in the decay of a $\pi^{+}$. The energy spectrum of these electrons is known very well, making them an excellent source for calibration. A Michel electron sample was obtained by selecting events with only small, isolated energy depositions. The energy spectrum of these events is compared with simulation in Fig. 5.28, The means of the distributions are shifted by 3\%, which is taken to be the error

on electromagnetic response. This error is used for energy deposited by $\gamma, \pi^{0}, e^{ \pm}$final state particles because the mechanism for energy loss is the same.

\section{Charged Mesons}

The uncertainty on charged pions comes from test beam measurements comparing the fraction of available energy that is observed in the detector in data and simulation [79]. The incoming energy is the precise beamline reconstruction. The observed energy is the sum of all hits in the detector corrected for the passive material traversed. Systematic errors on the simulation are considered, but an additional 5\% error is necessary to cover the discrepancy between data 


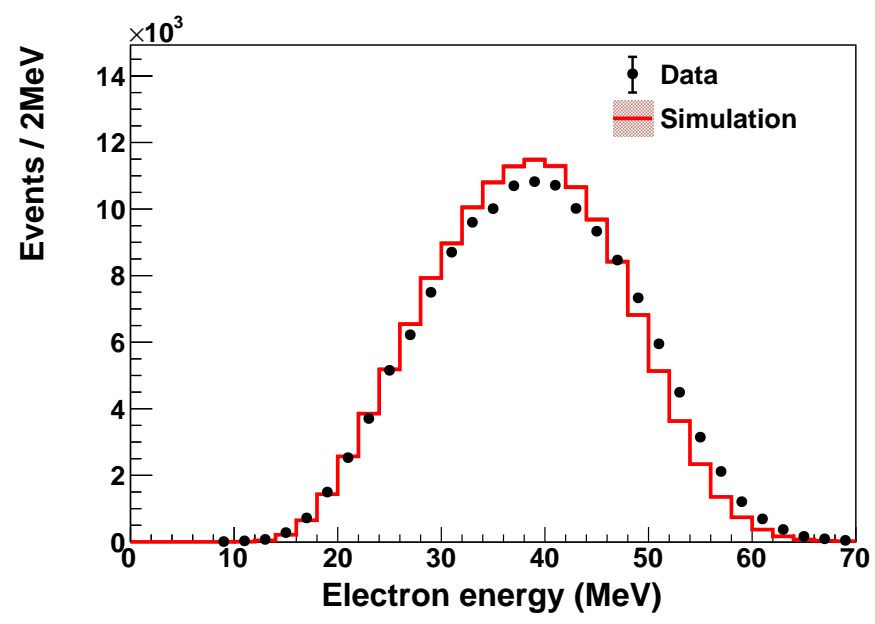

Figure 5.28: The energy spectrum of Michel electron candidates in data (points) and simulation (line). The means of the distributions agree to $3 \%$, which is taken as the uncertainty on the detector's response to electromagnetic particles. Figure from Ref. [26]

and simulation. $5 \%$ is then taken as the error on the detector response to charged pions. The result of the study is shown in Fig. 5.29. This uncertainty is also applied to kaon response, because their response is very similar and there are not sufficient statistics to perform a kaon-only measurement.

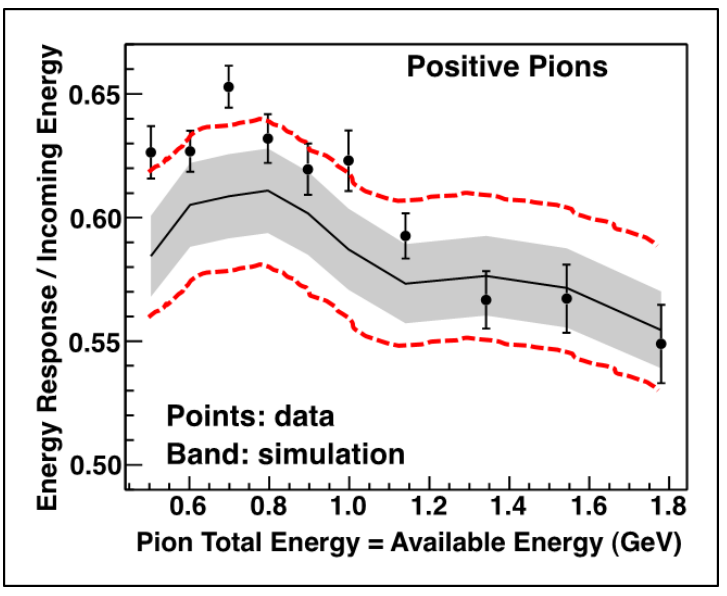

Figure 5.29: A comparison of the MINERvA test beam (T977) measurement for the detector's response to positive pions to simulation. The y-axis shows the visible energy corrected for traversed passive material divided by incoming pion energy measured by the beamline. The shaded simulation band shows the systematic errors. An additional error of 5\% is needed to cover the difference in data and simulation. The dotted red line approximates the systematic error on the simulation with the additional 5\% detector response error. Figure from Ref. [26]. 


\section{Cross-talk}

Cross-talk is simulated by specifying a likelihood that a recorded energy deposition will generate cross-talk in a neighboring channel [80]. The uncertainty on the detector response to cross-talk is dominated by optical rather than electrical cross-talk, because more than $90 \%$ of visible energy from cross-talk comes from the optical variety. Optical cross-talk was measured by two means: on a test stand with light injection and in-situ by looking at visible energy near rock muon tracks. These two methods disagree at the $20 \%$ level, which is the error on the detector response to cross-talk.

\subsubsection{Subtraction of Scintillator Contamination}

The subtraction of scintillator background described in Sec. 5.4 is data-driven, so all systematic errors discussed above apply to the scintillator background prediction; i.e. errors from flux, muon reconstruction, hadronic energy reconstruction, and normalization were evaluated on the distribution of events from the tracker used to predict the background. The convolution of migration due reconstruction systematic bias and reconstruction-dependent efficiency corrections was considered as well.

The statistical uncertainty in the true background distribution is accepted as a systematic error, because the prediction of scintillator background is normalized to the number of true background events. This error is uncorrelated among bins and among targets.

The predicted and true background distributions consistently compare at $1<\chi^{2} /$ d.o.f. when the above systematic errors are considered, which indicates the need for an additional systematic on the subtraction procedure itself. An additional systematic uncertainty is added so that the comparison of predicted and true backgrounds yields a $\chi^{2} /$ d.o.f. $\lesssim 1$. The $\chi^{2}$ calculation treats this additional systematic as uncorrelated bin-to-bin. Figure 5.30 demonstrates the determination of this systematic error. After it has been calculated, this systematic error is treated as correlated both bin-to-bin and target-to-target. This correlation is justified because 


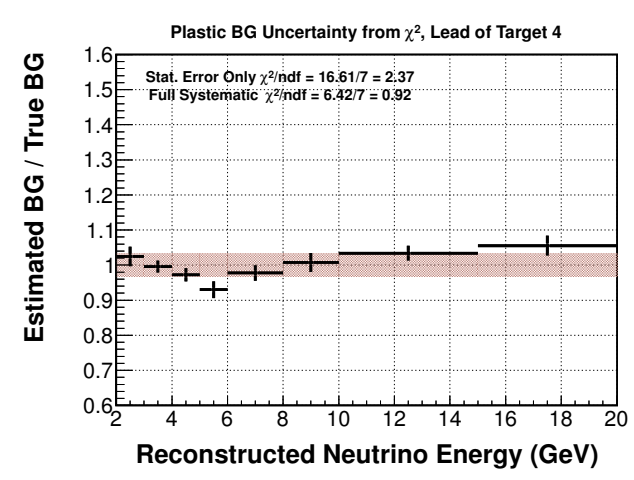

(a) Prediction of $E_{\nu}$ distribution

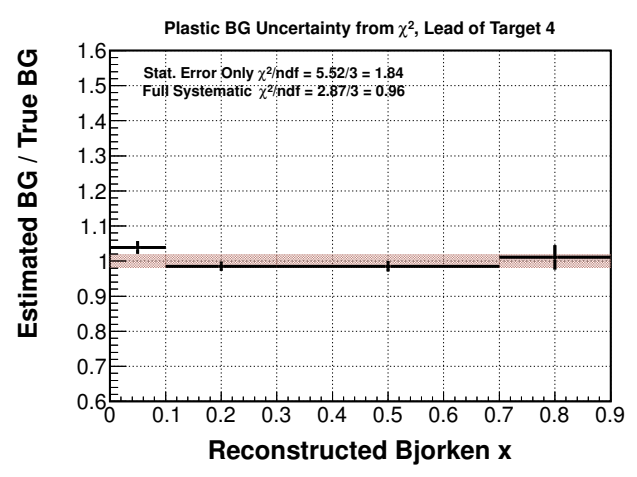

(b) Prediction of $x_{b j}$ distribution

Figure 5.30: The error on the $E_{h a d}$-dependent efficiency scale factor used to predict the scintillator background chosen to be sufficiently large for the true and estimated background distributions to agree within $\chi^{2} /$ d.o.f. $<1$. The tick marks show the ratio of estimated to true background with statistical error. The pink band about 1 shows the correlated systematic error on the $E_{h a d}$-dependent correction. $\chi^{2}$ calculations before and after the additional correlated error are shown in the figures.

the $E_{h a d}$-dependent scale factors have a similar shape for all passive targets.

\subsubsection{Unfolding}

The error on iterative Bayesian unfolding after iteration $i$ is equal to the difference in the distribution between iteration $i$ and $i+1[\overline{62}]$. This evaluation showed that the error on unfolding the neutrino energy spectrum is negligible, so it is ignored.

\subsubsection{Normalization}

Normalization errors comes from uncertainty in flat corrections to the data. Taking a ratio of cross sections results in a large cancellation of such uncertainties.

\section{Subtraction of Rock Muons}

Rock muons were a background in Target 2 for early data. The prevalence of these events was evaluated by scanning events displays, as discussed in Sec.5.3.2. Events were weighted to adjust for this background. The Gaussian approximation of the statistical error on identifying 
60 out of 975 events is $0.8 \%$. This error is for the integral number of events, but this analysis puts events into $\sim 9$ bins for kinematic variables. Therefore the error from rock muon weights is $\sqrt{(9)} \cdot 0.8 \%=2.4 \%$ and applies only to events in Target 2 from the earliest recorded data.

\section{Detector Mass}

Uncertainty in the mass of detector components translates into an error on the number of target nucleons. The uncertainty on the mass and chemical composition of the scintillator is $1.4 \%$ [26]. The uncertainty on the mass of passive targets is due to variations in density and thickness. Errors on the mass of carbon, iron, and lead are respectively $0.5 \%, 1.0 \%$, and $0.5 \%$. Uncertainty in mass is correlated for all events in a given nucleus and uncorrelated for events on different nuclear targets.

\subsubsection{Efficiency Correction}

\section{Muon Reconstruction Efficiency}

The muon reconstruction efficiencies in MINERvA and MINOS are described in Sec.5.5.2, The uncertainties on these corrections are the statistical error from the measurement of the efficiency, which are listed in Table 5.4 .

\section{Total Reconstruction Efficiency}

The full MINERvA Monte Carlo is used to evaluate the remaining reconstruction efficiency and geometric acceptance. All of the sources of systematic error described above apply to event selection used to form the efficiency.

Additionally, the use of a neutrino generator introduces cross section model dependence. The errors that come from cross section model uncertainties are evaluated by event reweighting. A cross section model parameter is changed within its uncertainty and the cross section for a

generated event is recalculated. The efficiency is calculated independently for $\pm 1 \sigma$ excursions 


\begin{tabular}{ccc}
\hline Model Parameter & Associated Process & $\delta(P) / P$ \\
\hline Axial mass for NC elastic & QE & $\pm 25 \%$ \\
Strange axial form factor $\eta$ for NC elastic & QE & $\pm 30 \%$ \\
Axial mass for CCQE & QE & $-15 \%,+25 \%$ \\
Normalization of CCQE & QE & $-15 \%,+20 \%$ \\
Pauli suppression in CCQE at low $Q^{2}$ & QE & $\pm 35 \%$ \\
CCQE vector form factor model & QE & BBA05 Dipole \\
Normalization of CC resonance production & Res & $\pm 20 \%$ \\
Normalization of NC resonance production & Res & $\pm 20 \%$ \\
Axial mass for CC resonance production & Res & $\pm 20 \%$ \\
Vector mass for CC resonance production & Res & $\pm 10 \%$ \\
Non-resonance CC1 $\pi$ production for $\nu p$ & DIS & $\pm 50 \%$ \\
Non-resonance CC2 $\pi$ production for $\nu p$ & DIS & $\pm 50 \%$ \\
Non-resonance CC1 $\pi$ production for $\nu n$ & DIS & $\pm 50 \%$ \\
Non-resonance CC $2 \pi$ production for $\nu n$ & DIS & $\pm 50 \%$ \\
$A_{H T}$ parameter in Bodek-Yang model & DIS & $\pm 25 \%$ \\
$B_{H T}$ parameter in Bodek-Yang model & DIS & $\pm 25 \%$ \\
$C_{V 1 u}$ parameter in Bodek-Yang model & DIS & $\pm 30 \%$ \\
$C_{V 2 u}$ parameter in Bodek-Yang model & DIS & $\pm 40 \%$ \\
\hline
\end{tabular}

Table 5.11: Uncertainties on neutrino cross section model in GENIE. This table is based on a similar table found in Ref. [24].

in each model parameter. Half of the maximum deviation among the excursions and central value calculations of efficiency is taken as the error due to that model parameter. The model parameters and their uncertainties are listed in Table 5.11. All model parameters are treated as independent and added in quadrature. The cross section model uncertainties are highly correlated among target samples; a model excursion which results in a larger cross section in lead is very likely to also give a higher cross section in all other nuclei. The cross section model uncertainties in the efficiency for the lead of Target 4 are shown in Fig. 5.31.

The statistical uncertainty on the Monte Carlo sample used to calculate efficiency is also a source of error in the efficiency correction. The statistical uncertainty is uncorrelated among events and targets.

Sources of error on the efficiency calculation for the lead of Target 4 are shown in Fig. 5.32. 

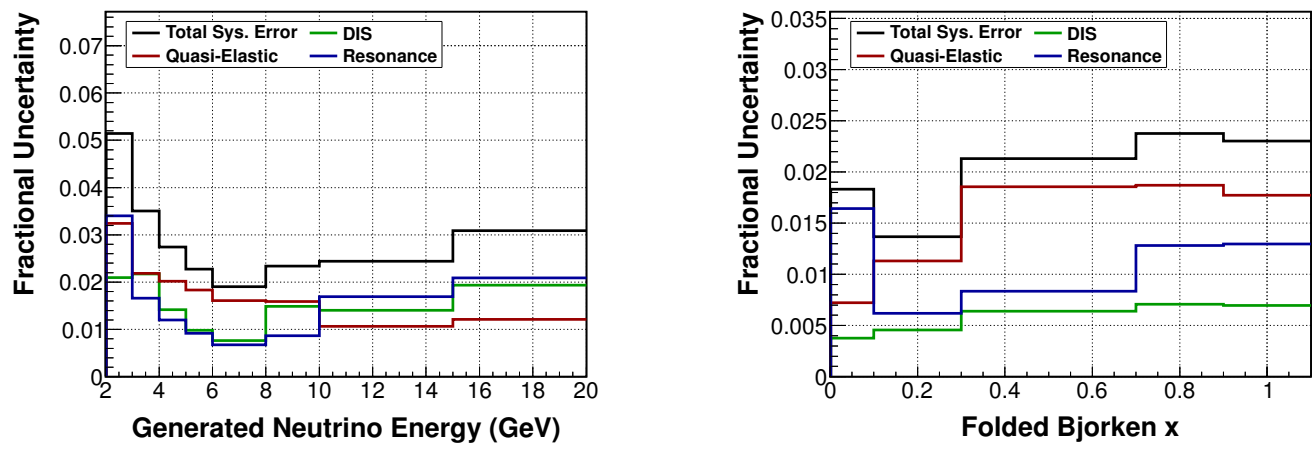

Figure 5.31: Relative errors on the reconstruction efficiency from uncertainty in cross section models. Model parameters are grouped by their associated interaction process. See Table 5.11 for the list of model parameters considered.
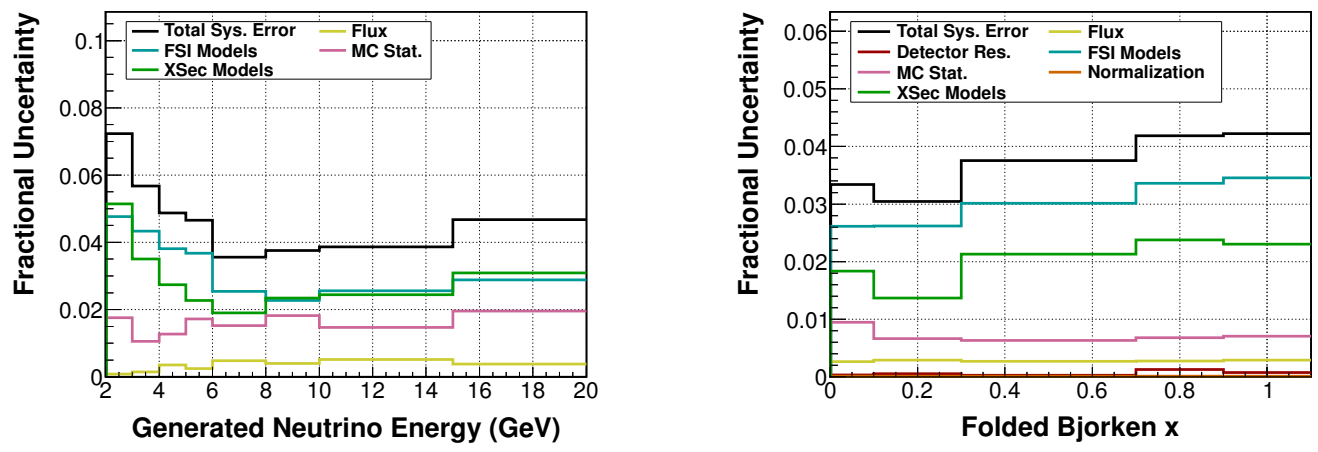

Figure 5.32: Summary of relative systematic errors on the reconstruction efficiency. 


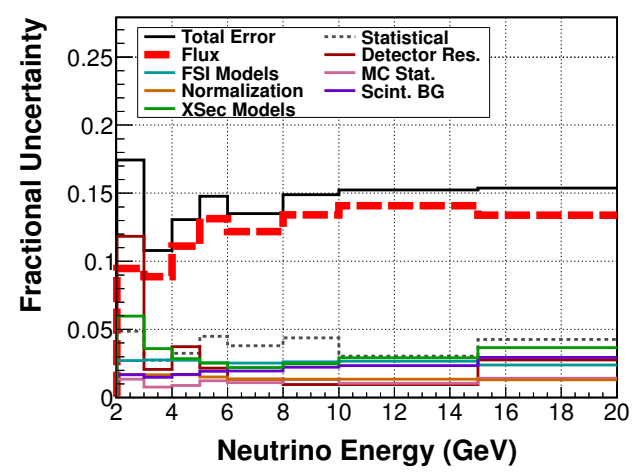

(a) $\sigma_{F e}$

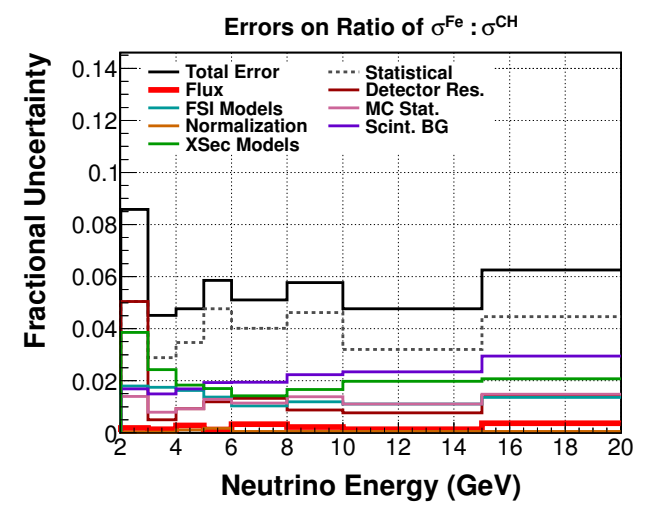

(c) $\sigma_{F e}: \sigma_{C H}$

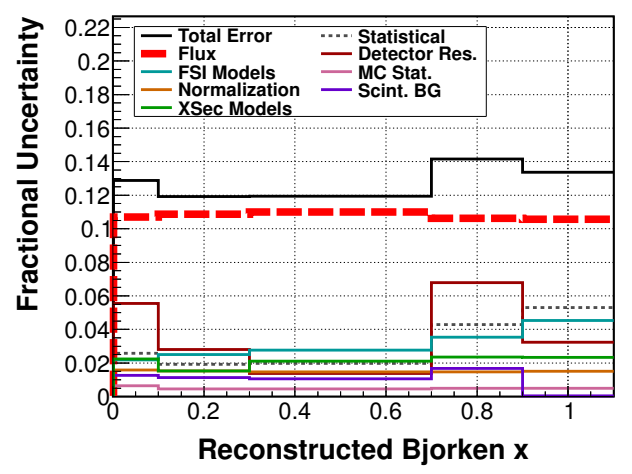

(b) $\frac{d \sigma_{F e}}{d x}$

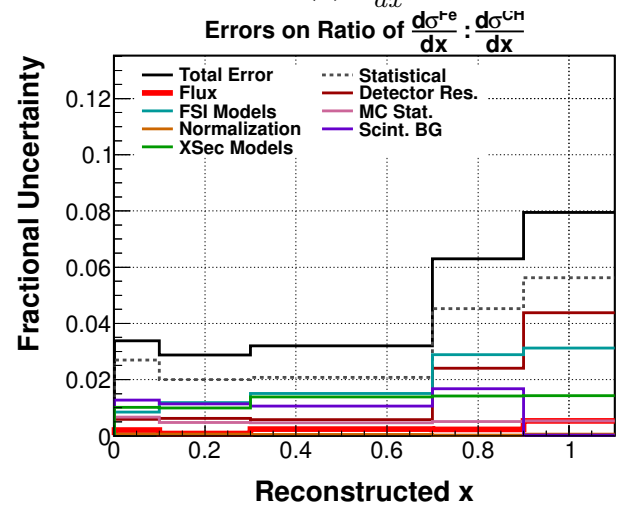

(d) $\frac{d \sigma_{F e}}{d x}: \frac{d \sigma_{C H}}{d x}$

Figure 5.33: Relative errors on the measurement of the iron absolute cross section (top) and ratio of iron to scintillator cross section (bottom). The flux error (bold red line) is obviously the dominant error in the absolute cross section measurement, but it is almost negligibly small in the measurement of cross section ratios.

\subsubsection{Cancellation of Errors in Ratios}

Many uncertainties on the measured cross section cancel or are greatly reduced through the use of ratios. The near cancellation of errors from the flux is the most beneficial reduction created by the ratio. A summary of errors for the absolute cross section of iron and the ratio of iron to scintillator cross sections is shown in Fig. 5.33. The relative error from the flux is highlighted to make its suppression more obvious. 


\section{Chapter 6}

\section{Results and Conclusions}

\subsection{Results}

The ratios of total charged current $\nu_{\mu}$ cross section $\sigma$ in carbon, iron, and lead to scintillator

are shown in Fig. 6.1 and Table 6.1. Results for $\frac{d \sigma}{d x_{b j}}$ are shown in Fig. 6.2 and Table 6.2 . Figure 6.3 shows the relative errors on these measurements. There are too many sources of systematic error to show in one plot, thus they have been merged into a small number of groups according to Table 6.3 .

\subsubsection{Neutrino Energy Result}

The agreement between data and Monte Carlo in Fig. 6.1 suggests that the nuclear dependence of the inclusive charged current $\nu_{\mu}$ cross section is well modeled as a function of neutrino energy. This is an important result for neutrino oscillation experiments, which use heavy nuclear targets. Poor modeling of nuclear effects in neutrino interactions would introduce large uncertainties in the shape of the neutrino energy spectra and, thus, result in less precise measurement of neutrino oscillation parameters.

\section{Limitations and Future Prospects}

While this result confirms the success of current models, there is still reason for oscillation searches to worry about the simulation of nuclear effects. The energy resolution and statistical limits of this analysis have limited the size of neutrino energy bins to $1 \mathrm{GeV}$. Oscillation 


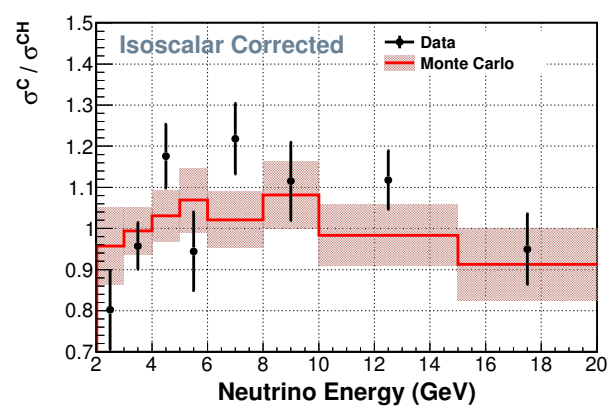

(a) Carbon

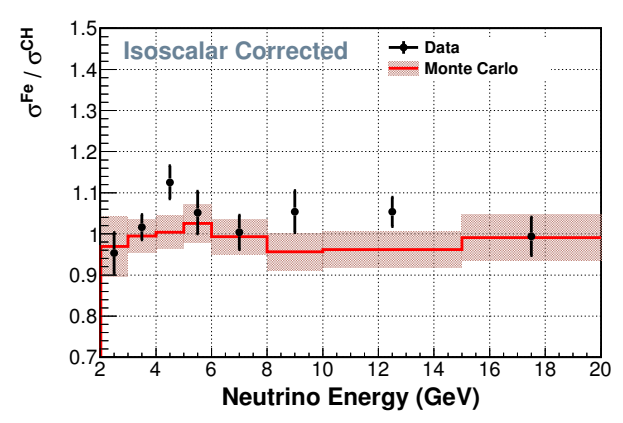

(c) Iron

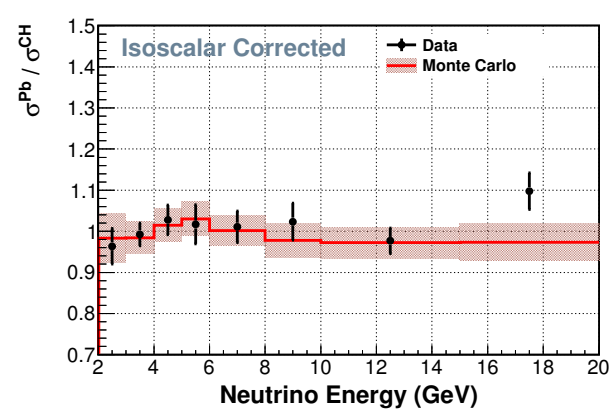

(e) Lead

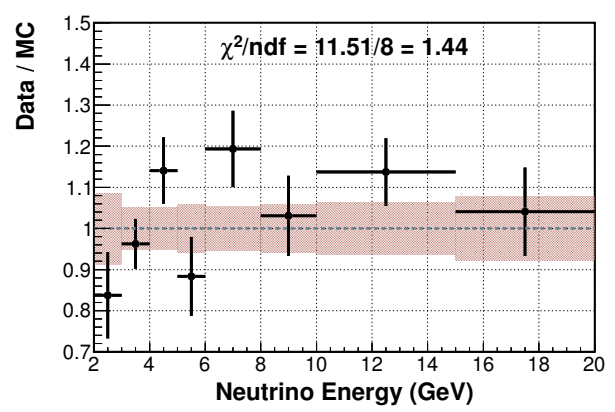

(b) Data to Monte Carlo ratio for carbon

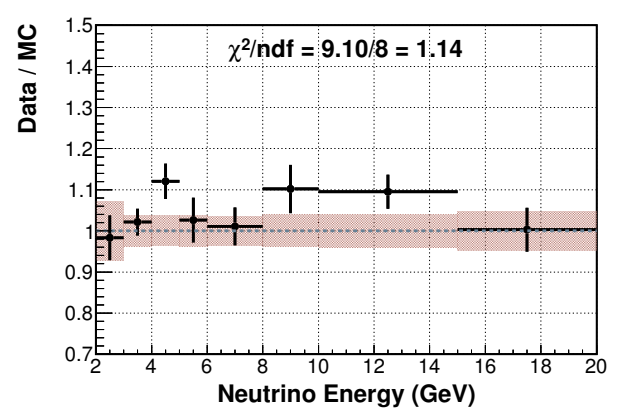

(d) Data to Monte Carlo ratio for iron

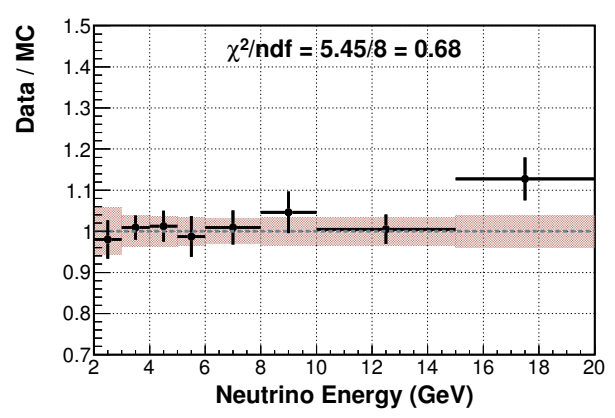

(f) Data to Monte Carlo ratio for lead

Figure 6.1: (Left) Results for the measurement of ratios of $\nu_{\mu}$ charged current inclusive $\sigma$ in carbon $(\mathrm{C})$, iron $(\mathrm{Fe})$, and lead $(\mathrm{Pb})$ to scintillator $(\mathrm{CH})$. (Right) The ratio of data to Monte Carlo of this result is also shown. Tick marks on data points show statistical error, and the pink band shows total systematic error. 


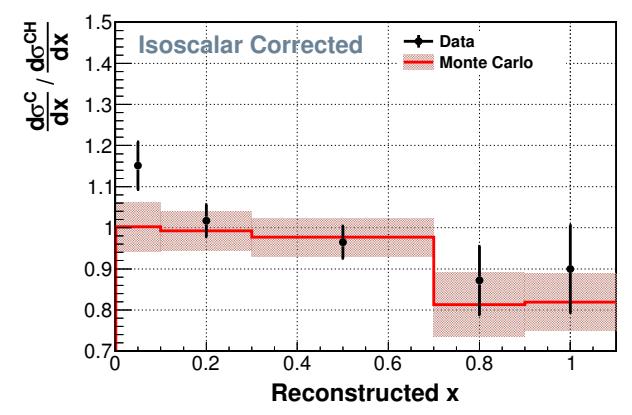

(a) Carbon

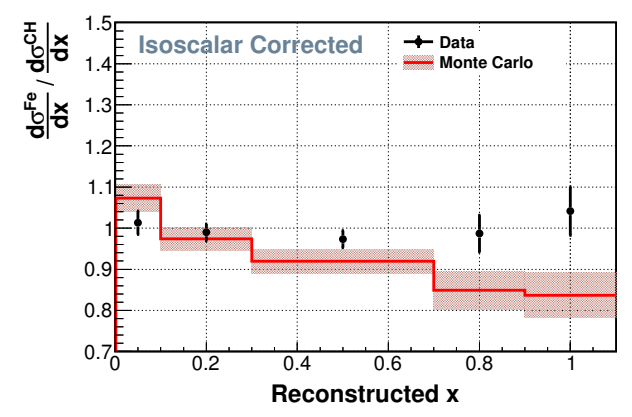

(c) Iron

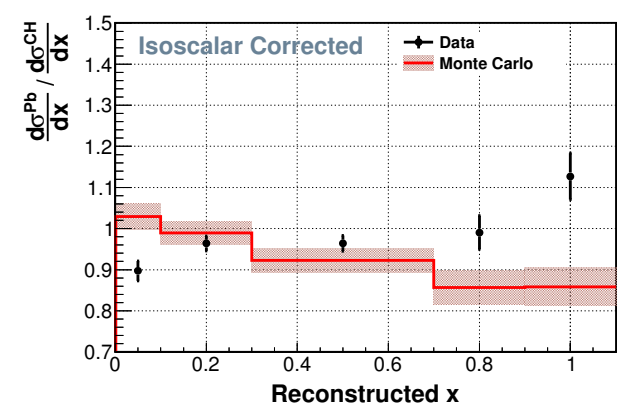

(e) Lead

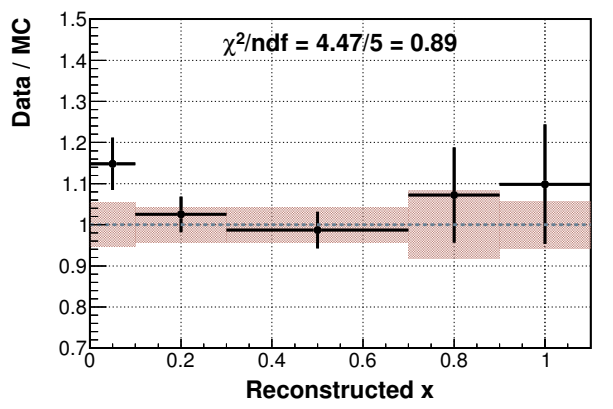

(b) Data to Monte Carlo ratio for carbon

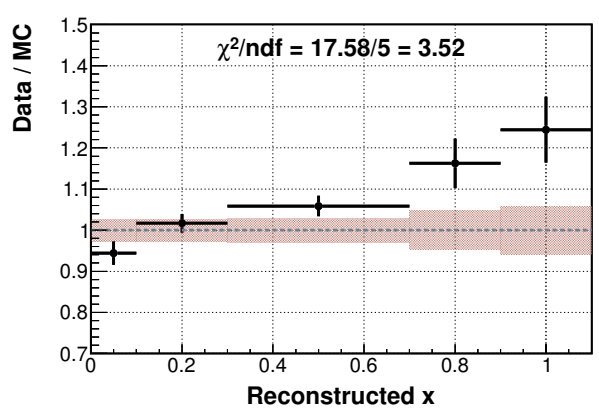

(d) Data to Monte Carlo ratio for iron

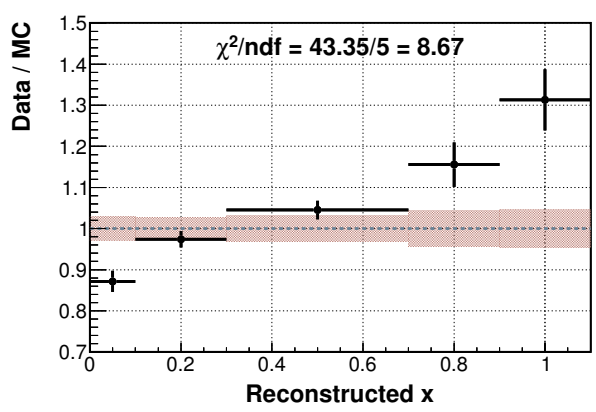

(f) Data to Monte Carlo ratio for lead

Figure 6.2: (Left) Results for the measurement of ratios of $\nu_{\mu}$ charged current inclusive $\frac{d \sigma}{d x_{b j}}$ in carbon $(\mathrm{C})$, iron $(\mathrm{Fe})$, and lead $(\mathrm{Pb})$ to scintillator $(\mathrm{CH})$. (Right) The ratio of data to Monte Carlo of this result is also shown. Tick marks on points shows statistical error, and the pink band shows total systematic error. 


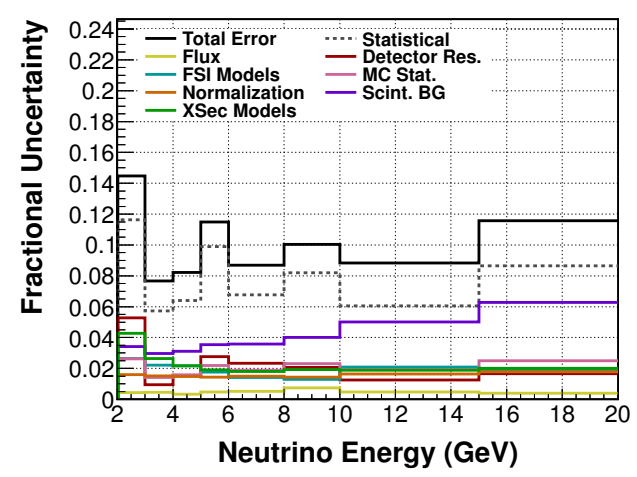

(a) Carbon $E_{\nu}$

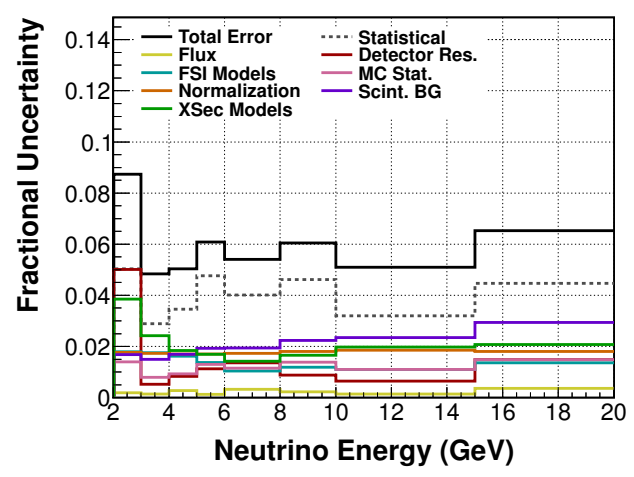

(c) Iron $E_{\nu}$

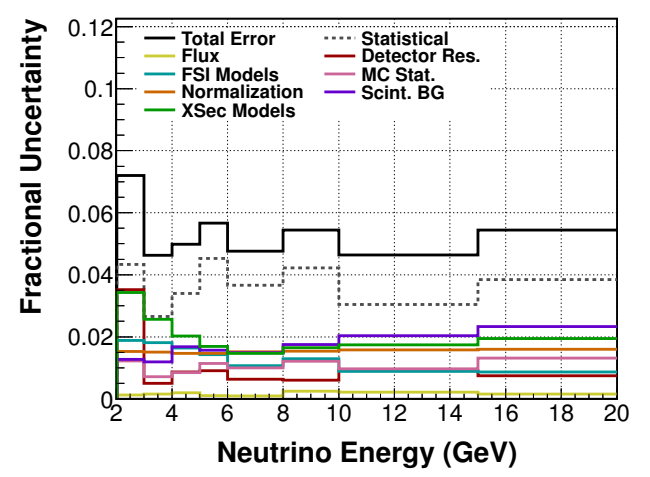

(e) Lead $E_{\nu}$

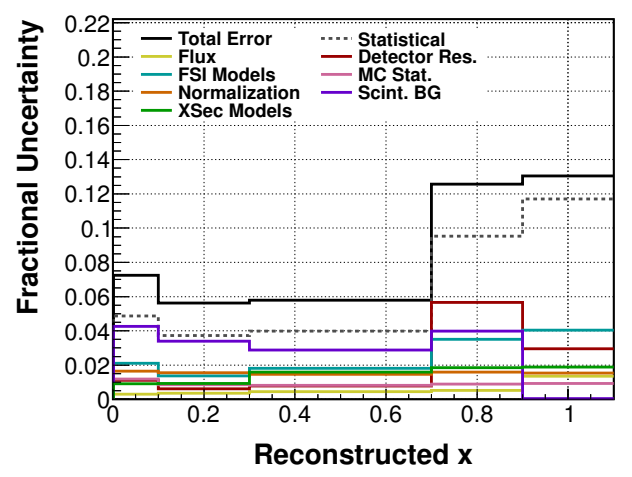

(b) Carbon $x_{b j}$

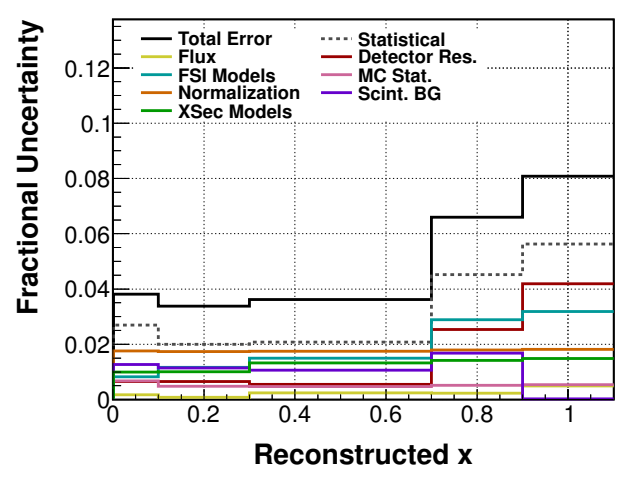

(d) Iron $x_{b j}$

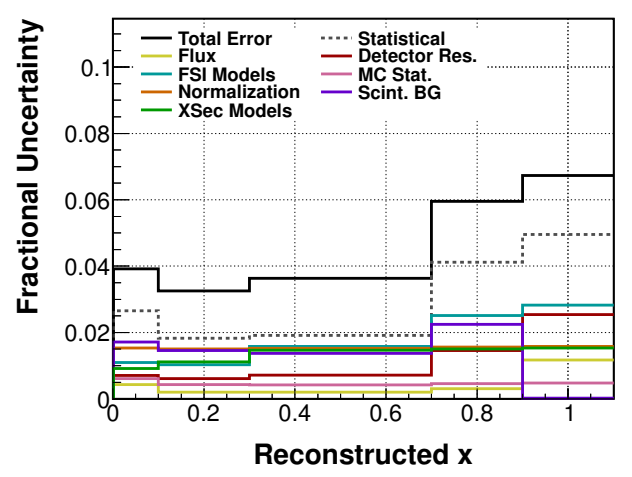

(f) Lead $x_{b j}$

Figure 6.3: Relative errors on ratios of $\sigma$ and $\frac{d \sigma}{d x_{b j}}$. 


\begin{tabular}{|c|c|c|c|c|c|c|}
\hline & $E_{\nu} \operatorname{Bin}(\mathrm{GeV})$ & Data & $\mathrm{MC}$ & $\sigma_{\text {stat }}$ & $\sigma_{\text {sys }}$ & $\frac{(\text { Data }-\mathrm{MC})}{\sigma_{t o t}}$ \\
\hline \multirow[t]{8}{*}{ Carbon } & $2-3$ & 0.80 & 0.96 & 0.093 & 0.069 & -1.25 \\
\hline & $3-4$ & 0.96 & 0.99 & 0.055 & 0.049 & -0.47 \\
\hline & $4-5$ & 1.18 & 1.03 & 0.075 & 0.060 & 1.43 \\
\hline & $5-6$ & 0.94 & 1.07 & 0.094 & 0.055 & -1.05 \\
\hline & $6-8$ & 1.22 & 1.02 & 0.082 & 0.066 & 1.75 \\
\hline & $8-10$ & 1.11 & 1.08 & 0.091 & 0.065 & 0.27 \\
\hline & $10-15$ & 1.12 & 0.98 & 0.068 & 0.072 & 1.27 \\
\hline & $15-20$ & 0.95 & 0.91 & 0.082 & 0.073 & 0.31 \\
\hline \multirow[t]{8}{*}{ Iron } & $2-3$ & 0.95 & 0.97 & 0.048 & 0.068 & -0.19 \\
\hline & $3-4$ & 1.02 & 0.99 & 0.029 & 0.039 & 0.42 \\
\hline & $4-5$ & 1.13 & 1.00 & 0.039 & 0.041 & 2.05 \\
\hline & $5-6$ & 1.05 & 1.03 & 0.050 & 0.040 & 0.39 \\
\hline & $6-8$ & 1.00 & 0.99 & 0.040 & 0.036 & 0.19 \\
\hline & $8-10$ & 1.05 & 0.96 & 0.049 & 0.041 & 1.42 \\
\hline & $10-15$ & 1.05 & 0.96 & 0.034 & 0.042 & 1.60 \\
\hline & $15-20$ & 0.99 & 0.99 & 0.044 & 0.047 & 0.04 \\
\hline \multirow[t]{8}{*}{ Lead } & $2-3$ & 0.96 & 0.98 & 0.042 & 0.055 & -0.27 \\
\hline & $3-4$ & 0.99 & 0.98 & 0.026 & 0.038 & 0.19 \\
\hline & $4-5$ & 1.03 & 1.02 & 0.035 & 0.037 & 0.23 \\
\hline & $5-6$ & 1.02 & 1.03 & 0.046 & 0.035 & -0.21 \\
\hline & $6-8$ & 1.01 & 1.00 & 0.037 & 0.031 & 0.18 \\
\hline & $8-10$ & 1.02 & 0.98 & 0.043 & 0.035 & 0.75 \\
\hline & $10-15$ & 0.98 & 0.97 & 0.030 & 0.034 & 0.10 \\
\hline & $15-20$ & 1.10 & 0.97 & 0.042 & 0.042 & 1.92 \\
\hline
\end{tabular}

Table 6.1: Comparison of measured to expected values for $\sigma^{A} / \sigma^{C H}$ for each neutrino energy bin, where $\mathrm{CH}$ is scintillator. Columns labeled as $\sigma$ show the errors on the cross section ratio. 


\begin{tabular}{ccccccc}
\hline & $x_{b j}$ Bin & Data & MC & $\sigma_{\text {stat }}$ & $\sigma_{\text {sys }}$ & $\frac{(\text { Data }-\mathrm{MC})}{\sigma_{\text {tot }}}$ \\
\hline Carbon & $0.0-0.1$ & 1.15 & 1.00 & 0.056 & 0.062 & 1.69 \\
& $0.1-0.3$ & 1.02 & 0.99 & 0.038 & 0.043 & 0.41 \\
& $0.3-0.7$ & 0.96 & 0.98 & 0.038 & 0.041 & -0.21 \\
& $0.7-0.9$ & 0.87 & 0.81 & 0.083 & 0.071 & 0.50 \\
& $0.9-1.1$ & 0.90 & 0.82 & 0.105 & 0.052 & 0.63 \\
\hline \multirow{6}{*}{ Iron } & $0.0-0.1$ & 1.01 & 1.07 & 0.027 & 0.027 & -1.46 \\
& $0.1-0.3$ & 0.99 & 0.97 & 0.020 & 0.027 & 0.46 \\
& $0.3-0.7$ & 0.97 & 0.92 & 0.020 & 0.029 & 1.47 \\
& $0.7-0.9$ & 0.99 & 0.85 & 0.045 & 0.047 & 2.01 \\
& $0.9-1.1$ & 1.04 & 0.84 & 0.059 & 0.060 & 2.32 \\
\hline \multirow{6}{*}{ Lead } & $0.0-0.1$ & 0.90 & 1.03 & 0.024 & 0.026 & -3.53 \\
& $0.1-0.3$ & 0.96 & 0.99 & 0.018 & 0.026 & -0.78 \\
& $0.3-0.7$ & 0.96 & 0.92 & 0.018 & 0.030 & 1.15 \\
& $0.7-0.9$ & 0.99 & 0.86 & 0.041 & 0.043 & 2.15 \\
& $0.9-1.1$ & 1.13 & 0.86 & 0.056 & 0.051 & 3.38 \\
\hline
\end{tabular}

Table 6.2: Comparison of measured to expected values for $\frac{d \sigma^{A}}{d x_{b j}} / \frac{d \sigma^{C H}}{d x_{b j}}$ for each $x_{b j}$ bin, where $\mathrm{CH}$ is scintillator. Columns labeled as $\sigma$ show the errors on the cross section ratio.

\begin{tabular}{cc}
\hline Group Name & Error Sources Included \\
\hline Detector Resolution & Muon energy, muon angle, hadronic energy, vertex position \\
FSI Models & All parameters described in Sec. 5.6.5 and 5.6 .5 \\
Cross Section Models (XSec) & All model parameters described in Table 5.11 \\
MC Statistics & Statistics of Monte Carlo used in to calculate efficiency \\
Normalization & Muon tracking efficiencies, mass, rock muon rate \\
Flux & All sources of flux uncertainty \\
Scintillator Background & Statistical and scale errors on scintillator background \\
\hline
\end{tabular}

Table 6.3: Grouping of systematic errors for display purposes. 
experiments usually require finer bins to precisely measure the neutrino energy spectrum near the expected region of (dis)appearance. For example, MINOS uses $0.5 \mathrm{GeV}$ bins. It is possible that any unmodeled behavior in nuclear dependence is observable only at this finer granularity. Many neutrino oscillation experiments operate at energies slightly lower than those measured in this analysis. Lower energy experiments often attempt to isolate the quasi-elastic channel, which is the dominant channel for neutrino energies below $3 \mathrm{GeV}$. The manifestations of nuclear dependence are different for elastic and inelastic interaction channels, and it is possible that the inclusive measurement will dampen these manifestations.

MINERvA is working to address each of these limitations. The energy resolution of the detector will improve through better reconstruction and further analysis of data taken by the MINERvA Test Beam Collaboration (E977). This analysis used only muons that were analyzed in the MINOS spectrometer. Including events in which the muon stops in MINERvA will extend the analysis to lower neutrino energies and greatly improve the statistics of the measurement. MINERvA has already presented an analysis of quasi-elastic events in the tracker region and is developing an analysis of quasi-elastic events on the nuclear targets.

\subsubsection{Bjorken x Result}

This result shows significant disagreement with Monte Carlo at both low and high values

of $x_{b j}$. The discrepancy is a suppression (enhancement) at low (high) $x_{b j}$ that grows with the size of the nucleus.

One possible explanation for the difference is imperfect simulation of detector smearing. This may occur because of improper simulation or optimistic accounting of uncertainties of final state interactions (FSI). Take an increase in the cross section for intranuclear pion absorption as a hypothetical change to FSI. This would lead to fewer pions exiting the nucleus and thus a lower observed hadronic energy $\nu$. In turn, this would distort the $x_{b j}$ distribution to higher values because $x_{b j}=Q^{2} / 2 M \nu$. Such an effect would be more pronounced for larger 
nuclei, thus introducing a nuclear-dependent effect. It is very difficult to disentangle nuclear dependence in cross sections from FSI; however, it may be possible by performing measurements of nuclear dependence using different exclusive final states. MINERvA is capable of making such measurements with great precision, but it is not clear that it is even theoretically possible to disentangle the two effects.

Discussions specific to the physics of low and high $x_{b j}$ are below.

\section{Low Bjorken $x$}

The data show a suppression in the cross section ratio at low $x_{b j}\left(x_{b j}=[0.0,0.1]\right)$ that grows with the size of the nucleus. This is not predicted by the simulation. At low $x_{b j}$, shadowing and antishadowing effects modify the structure function $F_{2}$. Shadowing is understood to be caused by the phenomenon of quark multiple scattering, which produces a suppression at $x_{b j}<0.07$, where the value of $x_{b j}$ at the turnover point varies with the nucleus and level of contribution from the axial current [13]. The cause of the enhancement of $F_{2}$ in the antishadowing region is not understood, but the effect has been conclusively observed. These effects are well-measured for vector $F_{2}$ from charged lepton scattering, but have not been directly measured in neutrino scattering.

The events used for this measurement have are well into the nonperturbative regime ( $80 \%$ of the events have $Q^{2}<1(\mathrm{GeV} / c)^{2}, 60 \%$ have $\left.Q^{2}<0.5(\mathrm{GeV} / c)^{2}\right)$. Nuclear modification of structure functions is normally measured in the perturbative regime $\left(Q^{2}>1(\mathrm{GeV} / c)^{2}\right)$, where more precise calculations are possible. Shadowing effects are known to be enhanced at lower values of $Q^{2}$. Thus there is reason to believe that the modifications measured in this analysis should be more extreme than measurements made with DIS events in safely perturbative kinematic space.

Most simulations of neutrino scattering use the very same modifications observed in charged 
lepton scattering, but standard theories say this cannot be correct. Neutrino scattering amplitudes are sensitive to $x F_{3}$ and the axial structure of $F_{2}$, which is not the case in charged lepton scattering. $F_{2}$ can have quite different behavior for neutrino and charged lepton scattering at low $Q^{2}$. Vector contributions to the cross section, which are the only type that exist for charged lepton scattering experiments, go to zero as $Q^{2} \rightarrow 0$. The axial contributions do not vanish. We expect that there are differences in the nuclear modification of structure functions for neutrinos and charged leptons, and that these differences are larger at low $Q^{2}$.

\section{High Bjorken $x$}

Events at high $x_{b j}\left(x_{b j}=[0.7,1.1]\right)$ come predominantly from the quasi-elastic channel. The nuclear dependence of quasi-elastic neutrino scattering is not well simulated. This topic has been of great interest in the field for the last few years.

The quasi-elastic cross section is usually simulated using a dipole for the axial form factor, which has just one parameter: the axial mass $M_{A}$. High precision measurements of $M_{A}$ using quasi-elastic neutrino scattering off nuclei made in last decade show significant tension. For example, an analysis of NOMAD data at $\left\langle E_{\nu}\right\rangle=25 \mathrm{GeV}$ yields $M_{A}=1.05 \pm 0.06 \mathrm{GeV} / c^{2}$, in agreement with a fit to world data from free nucleon targets; while MiniBooNE data at $<E_{\nu}>=1 \mathrm{GeV}$ yields $M_{A}=1.350 \pm 0.066 \mathrm{GeV} / c^{2}$. Both analyses are performed using a relativistic Fermi gas (RFG) model of the nucleus, in which the nuclear cross section is an incoherent sum of free nucleon cross sections. This is the same nuclear model used by MINERvA. However, electron elastic scattering measurements have shown that nucleonnucleon correlations result in an increase of up to $20 \%$ in the transverse cross section, so-called "transverse enhancement" [21, 81, 82, 83]. The expected energy dependence of transverse enhancement explains a significant amount of the disagreement between the MiniBooNE and NOMAD measurements of $M_{A}$ [21]. It is possible that some of the disagreement between the data and Monte Carlo of Fig. 6.2 at high $x_{b j}$ is due to the nuclear dependence of transverse 
enhancement in quasi-elastic channel.

\subsection{Conclusions}

Ratios of the inclusive charged current $\nu_{\mu}$ cross section on carbon, iron, and lead to scintilla-

tor have been measured as a function of neutrino energy $(\sigma)$ and $x_{b j}\left(\frac{d \sigma}{d x_{b j}}\right)$. The measurement was restricted to $2 \mathrm{GeV}<E_{\nu}<20 \mathrm{GeV}$ and $0^{\circ}<\theta_{\mu}<17^{\circ}$ due to geometric limitations of the muon spectrometer. These results are the first precision measurement of the nuclear dependence of the neutrino inclusive cross section in the few-GeV neutrino energy regime, and the first measurement of nuclear modification of structure functions in neutrino scattering in the nonperturbative regime. This is the first analysis of events on the nuclear targets in MINERvA, and also the first measurement of inclusive cross sections in MINERvA.

The results show an assuring, though not definitive, confirmation that the nuclear dependence of $\sigma$ is modeled well for neutrino energy distributions. This is important to measurements of oscillation parameters like mass splitting and mixing angles, because these parameters are measured on heavy targets through analysis of the neutrino energy spectra. It is important to improve the energy resolution of this measurement and to probe lower energies in order to provide oscillation searches with a better constraint.

The measurement of the nuclear dependence of $\frac{d \sigma}{d x_{b j}}$ shows behavior that is not modeled well at both low and high $x_{b j}$. Possible explanations include (1) enhanced final state interactions, (2) stronger than expected shadowing, and (3) transverse enhancement of the quasielastic cross section. Measurements of the nuclear dependence of exclusive channels are likely necessary to explain the observed deviations from expectation. Future MINERvA analyses of the nuclear dependence of deep inelastic scattering can use data collected with the recently upgraded NuMI beam at higher energies to make more direct measurements of the effective nuclear modification of structure functions. A MINERvA analysis of the nuclear dependence of quasi-elastic scattering is in development and will directly address the discrepancy at high 
$x_{b j}$. 


\section{Appendix A}

\section{Additional Figures}

\section{A.1 Kinematic Distributions of Selected Events}

Figures in this section show distributions of reconstructed kinematic variables for the charged current $\nu_{\mu}$ event samples. The simulation is split into stacked histograms based on the interaction type as follows:

- CC-QE - Yellow - Charged current quasi-elastic.

- $W<1.3(\mathrm{GeV})$ inelastic - Orange - Resonances with low invariant mass.

- $1.3<W<2(\mathrm{GeV})-\mathrm{Red}-$ The transition region from resonance to deep inelastic scattering.

- $Q^{2}<1\left(\mathrm{GeV} / c^{2}\right), W>(2 \mathrm{GeV})$ - Pink — Not-so-deep inelastic scattering (nonperturbaitve DIS).

- $Q^{2}>1\left(\mathrm{GeV} / c^{2}\right), W>(2 \mathrm{GeV})$ - Purple - Safe deep inelastic scattering (DIS).

Neutral current and wrong sign events are excluded. The simulation has been area-normalized by the number of events which pass the event selection. The data points show statistical uncertainty. Each page shows a different event sample. 

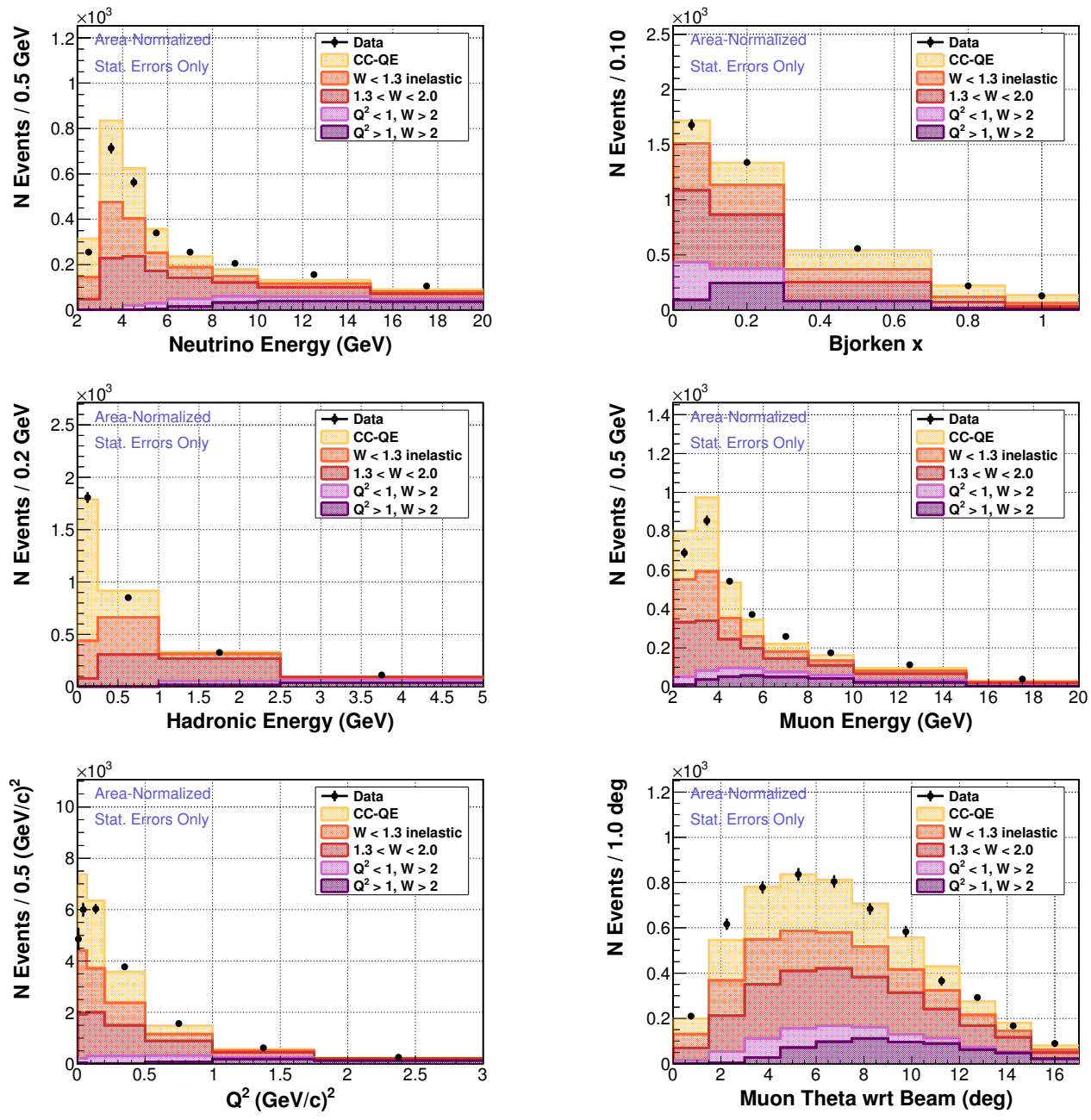

(a) Target 2 iron

Figure A.1: Kinematic distributions of selected events. For explanation, see top of this section. 

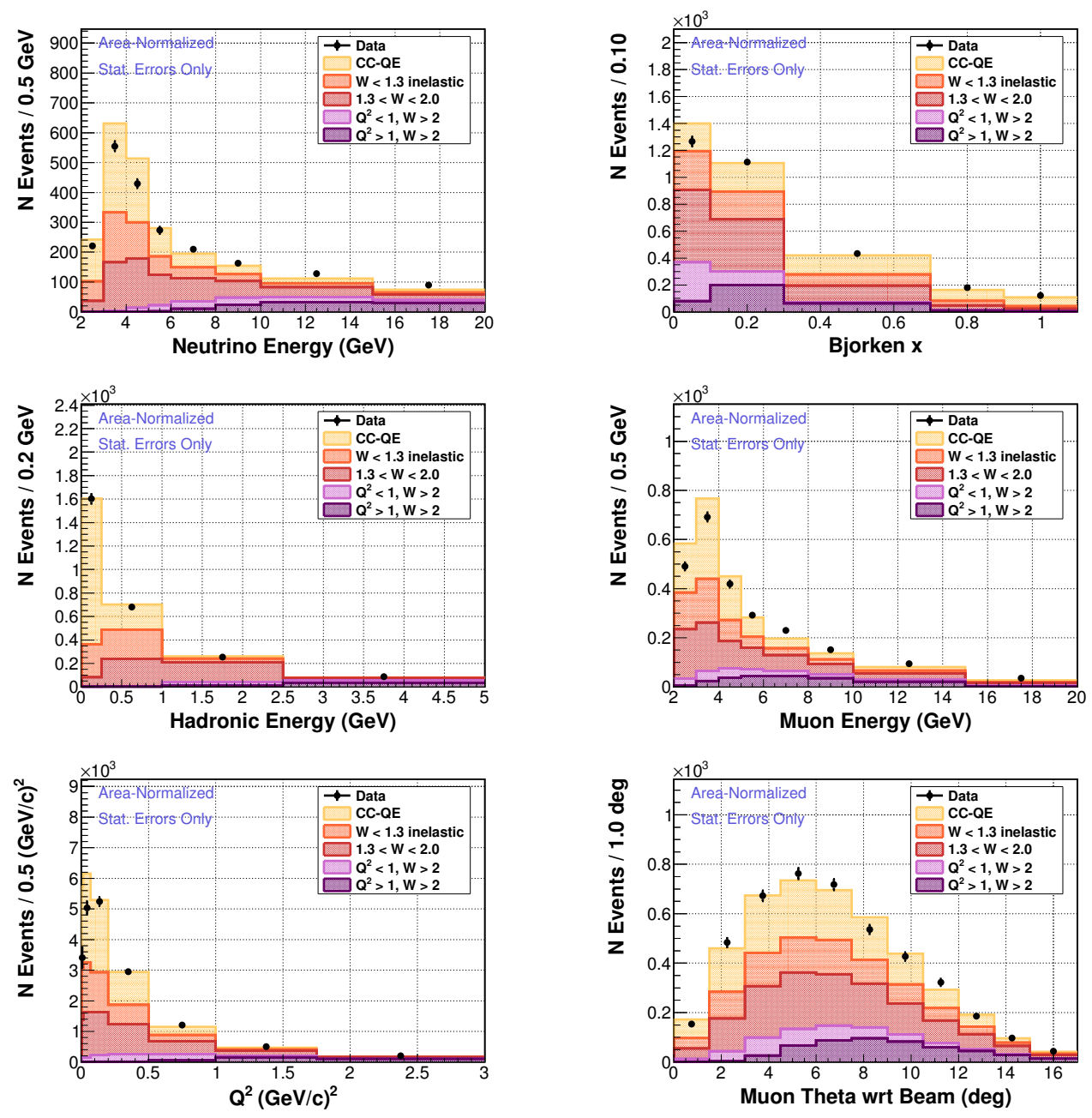

(b) Target 2 lead

Figure A.1: Kinematic distributions of selected events. For explanation, see top of this section. 

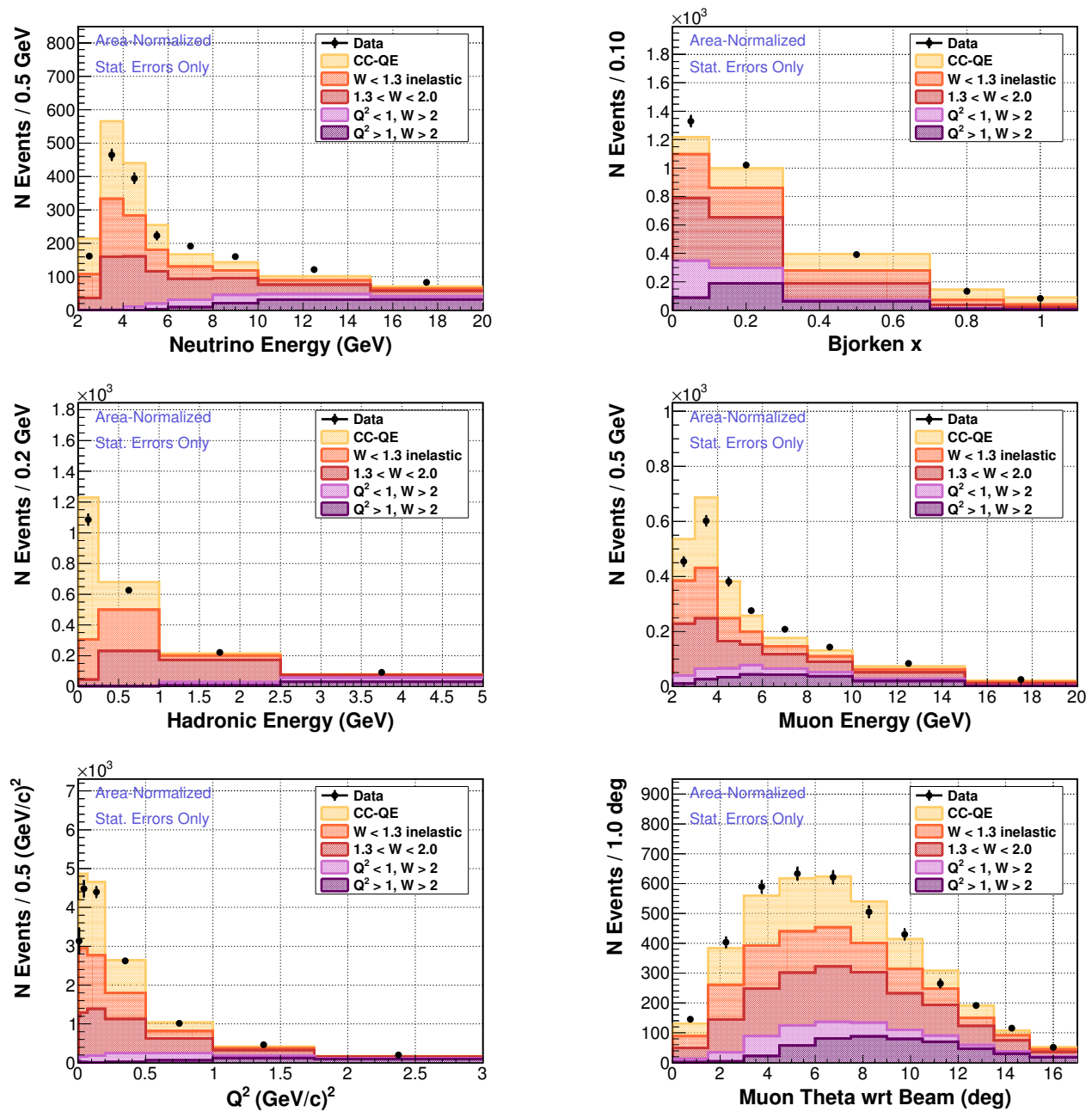

(c) Target 3 carbon

Figure A.1: Kinematic distributions of selected events. For explanation, see top of this section. 

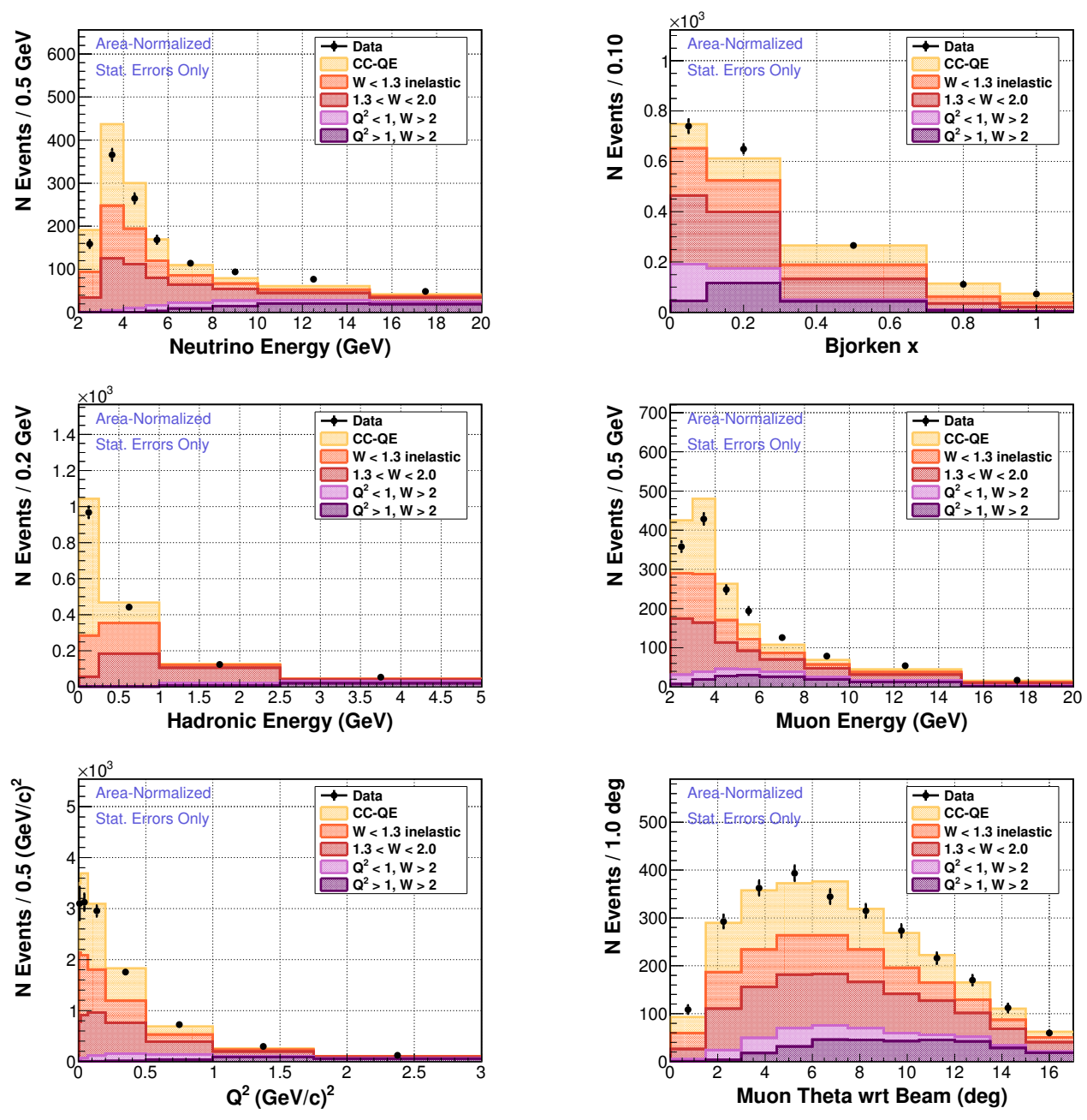

(d) Target 3 iron

Figure A.1: Kinematic distributions of selected events. For explanation, see top of this section. 

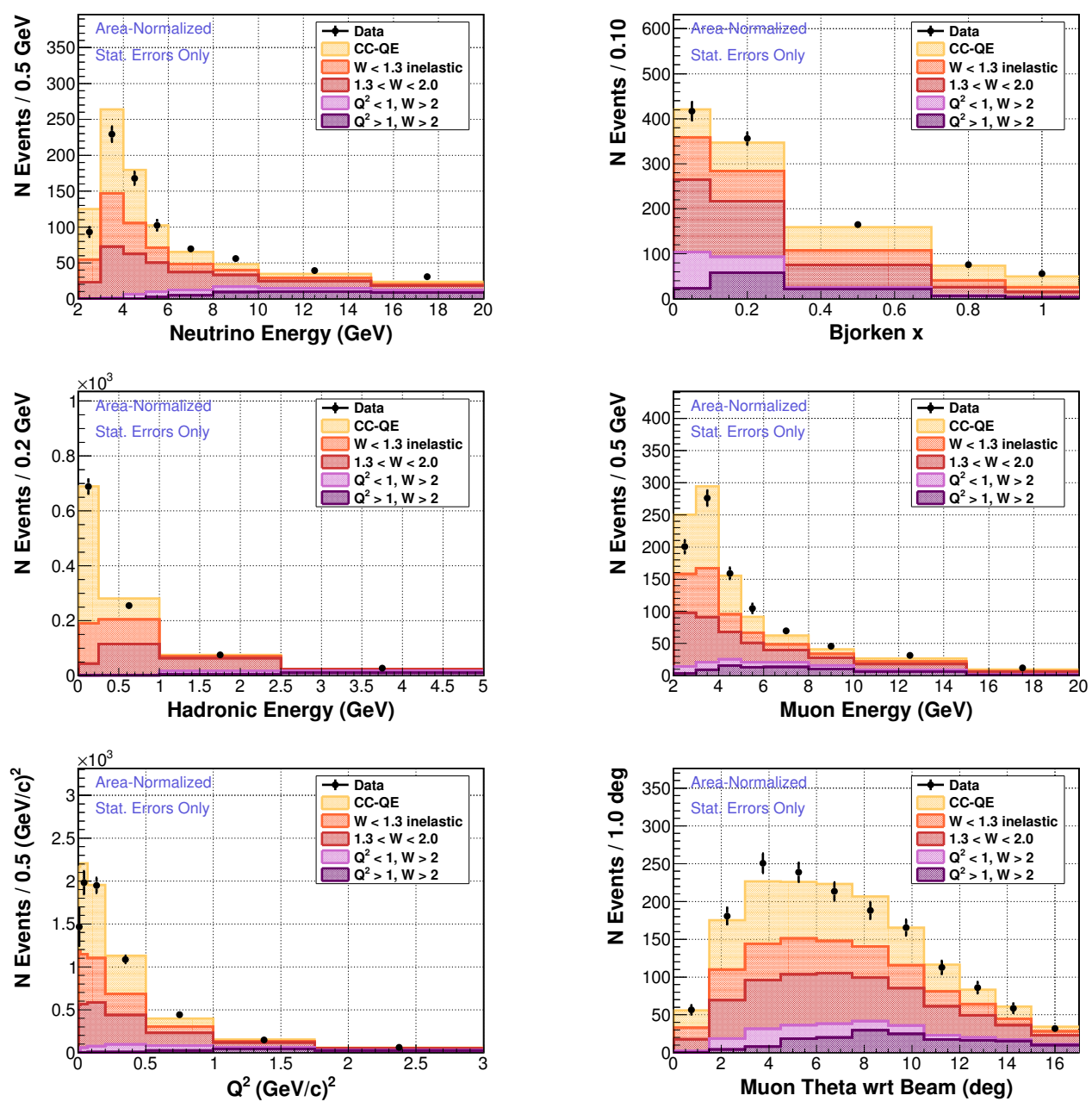

(e) Target 3 lead

Figure A.1: Kinematic distributions of selected events. For explanation, see top of this section. 

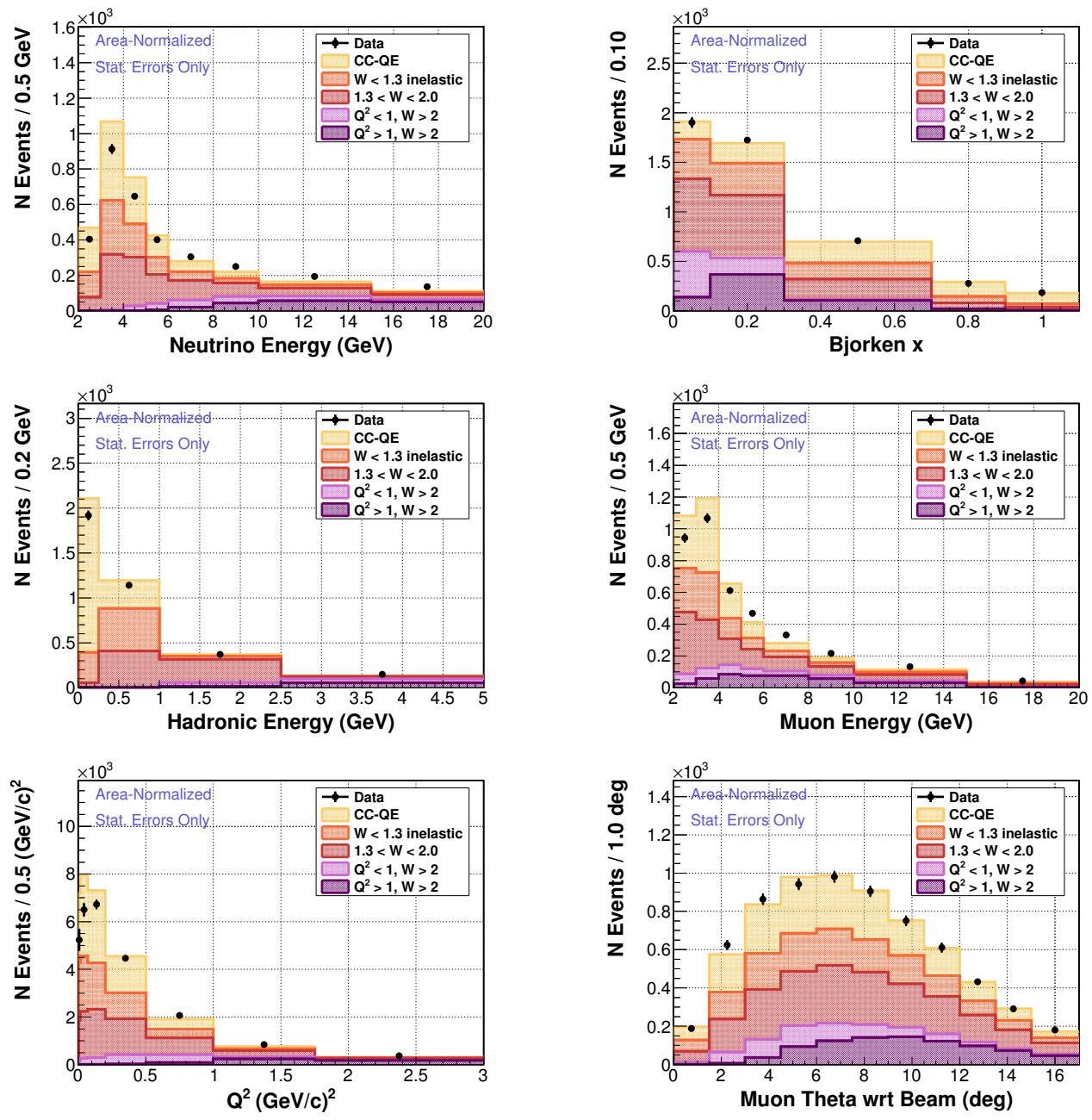

(f) Target 4 lead

Figure A.1: Kinematic distributions of selected events. For explanation, see top of this section. 

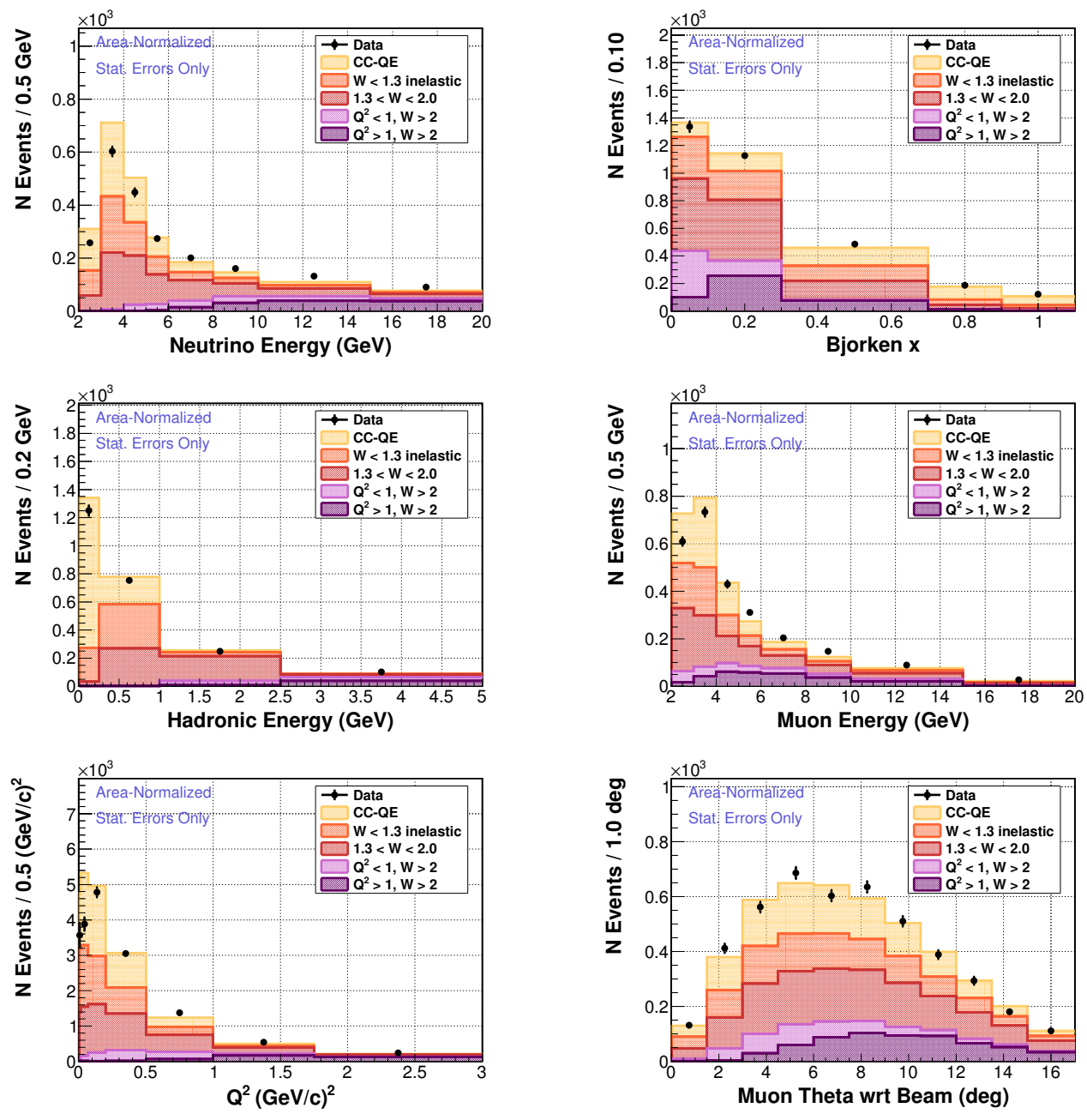

(g) Target 5 iron

Figure A.1: Kinematic distributions of selected events. For explanation, see top of this section. 

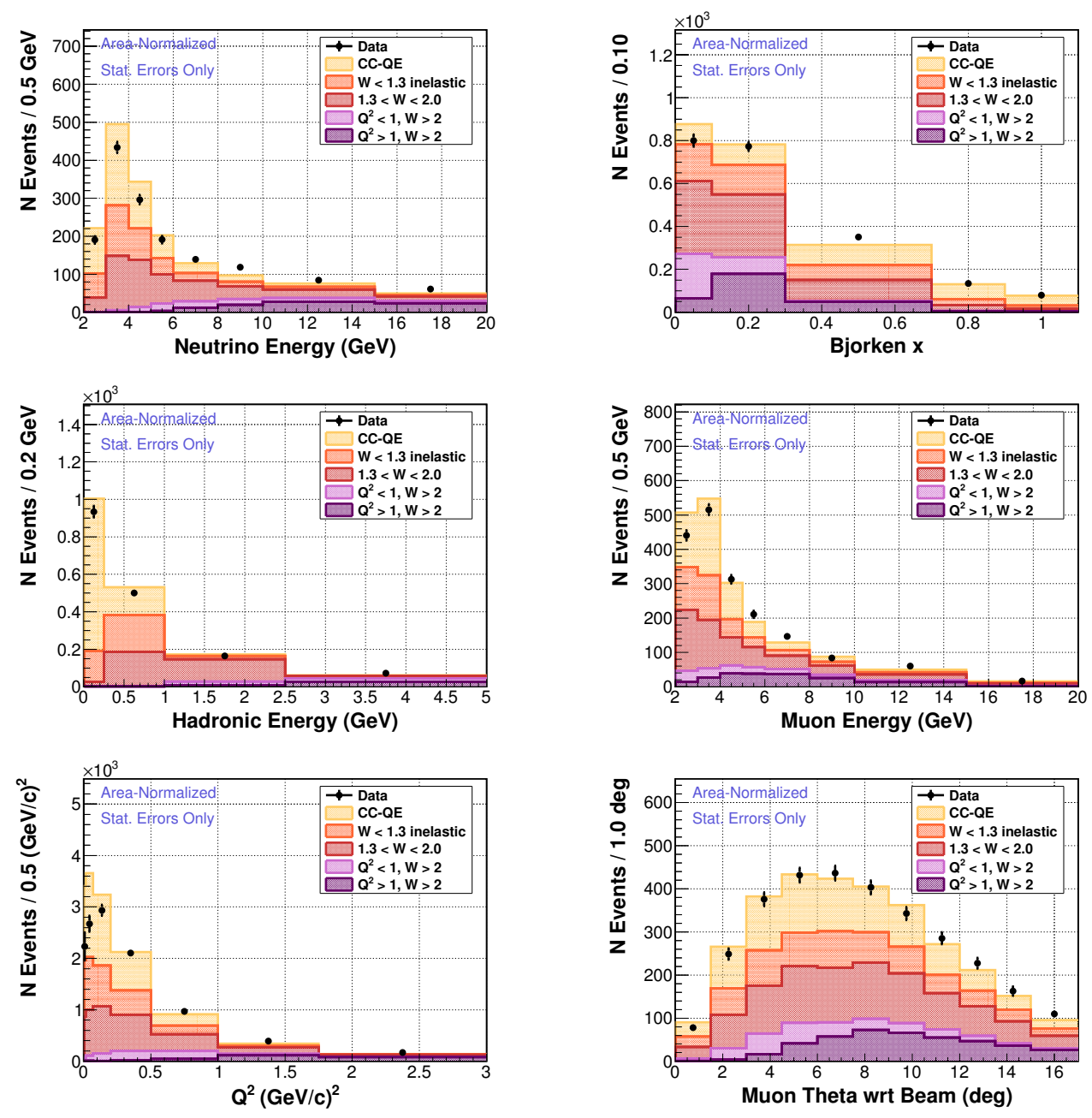

(h) Target 5 lead

Figure A.1: Kinematic distributions of selected events. For explanation, see top of this section. 

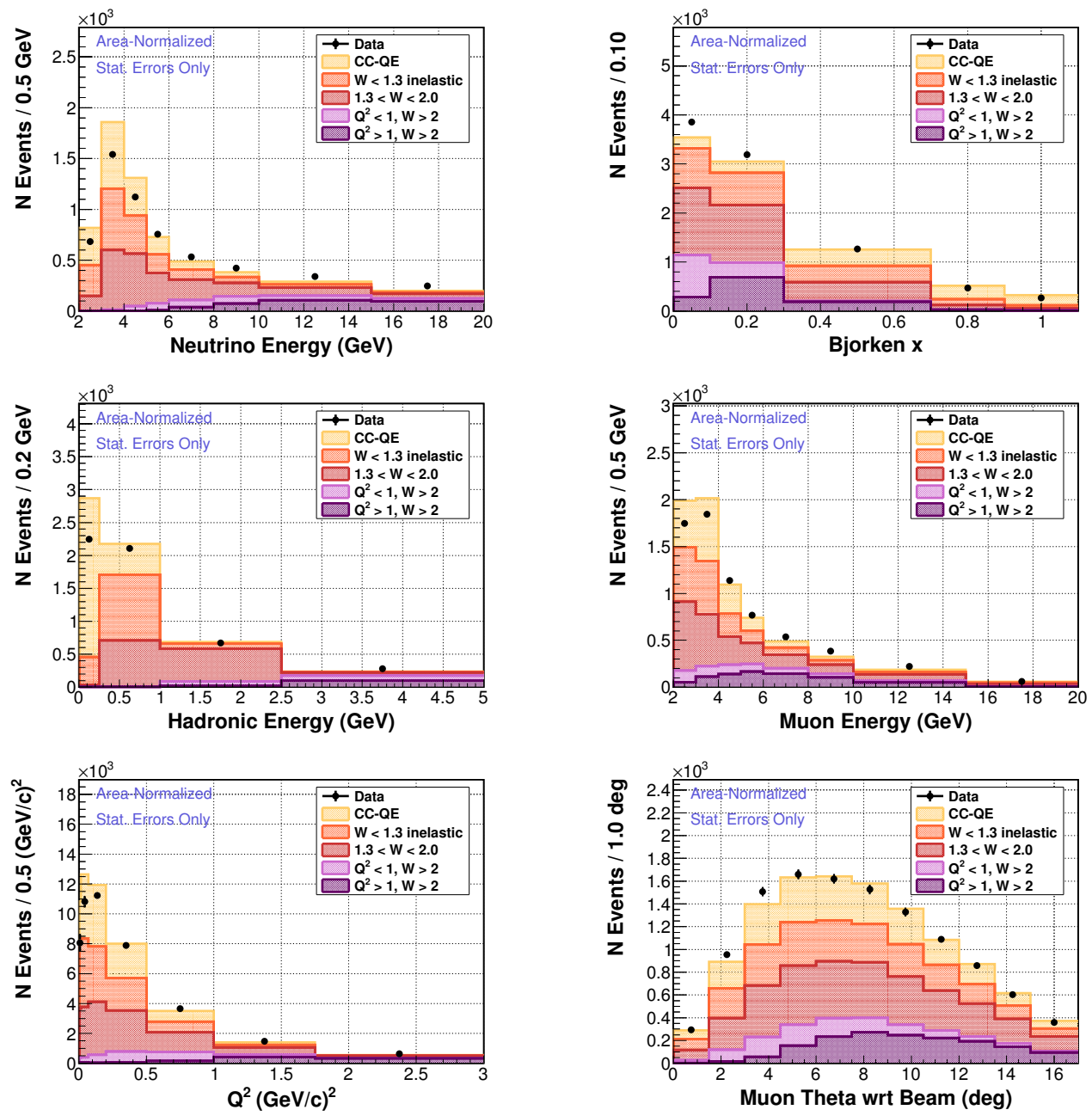

(i) Tracker modules 27-32

Figure A.1: Kinematic distributions of selected events. For explanation, see top of this section. 

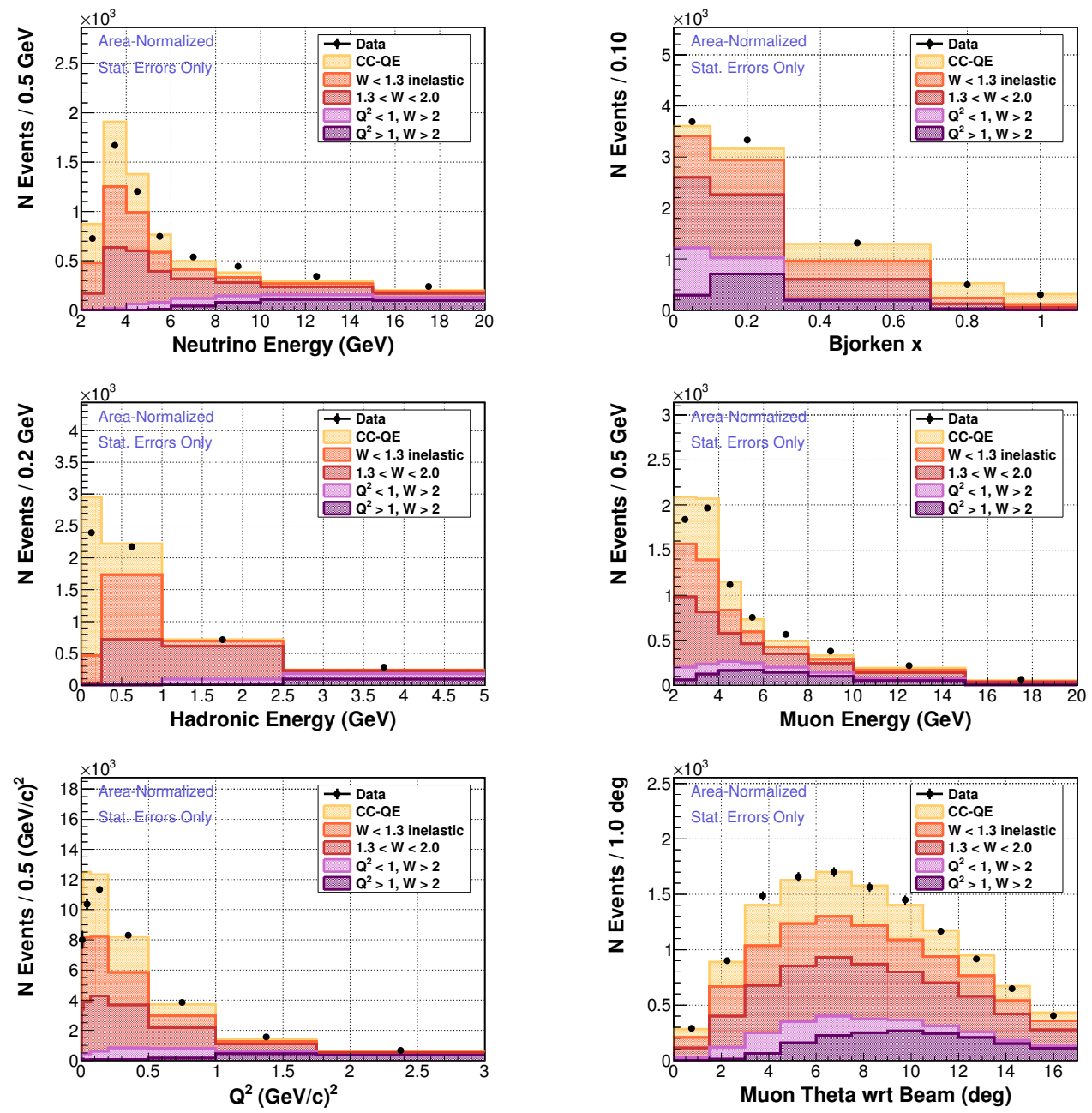

(j) Tracker modules 33-38

Figure A.1: Kinematic distributions of selected events. For explanation, see top of this section. 

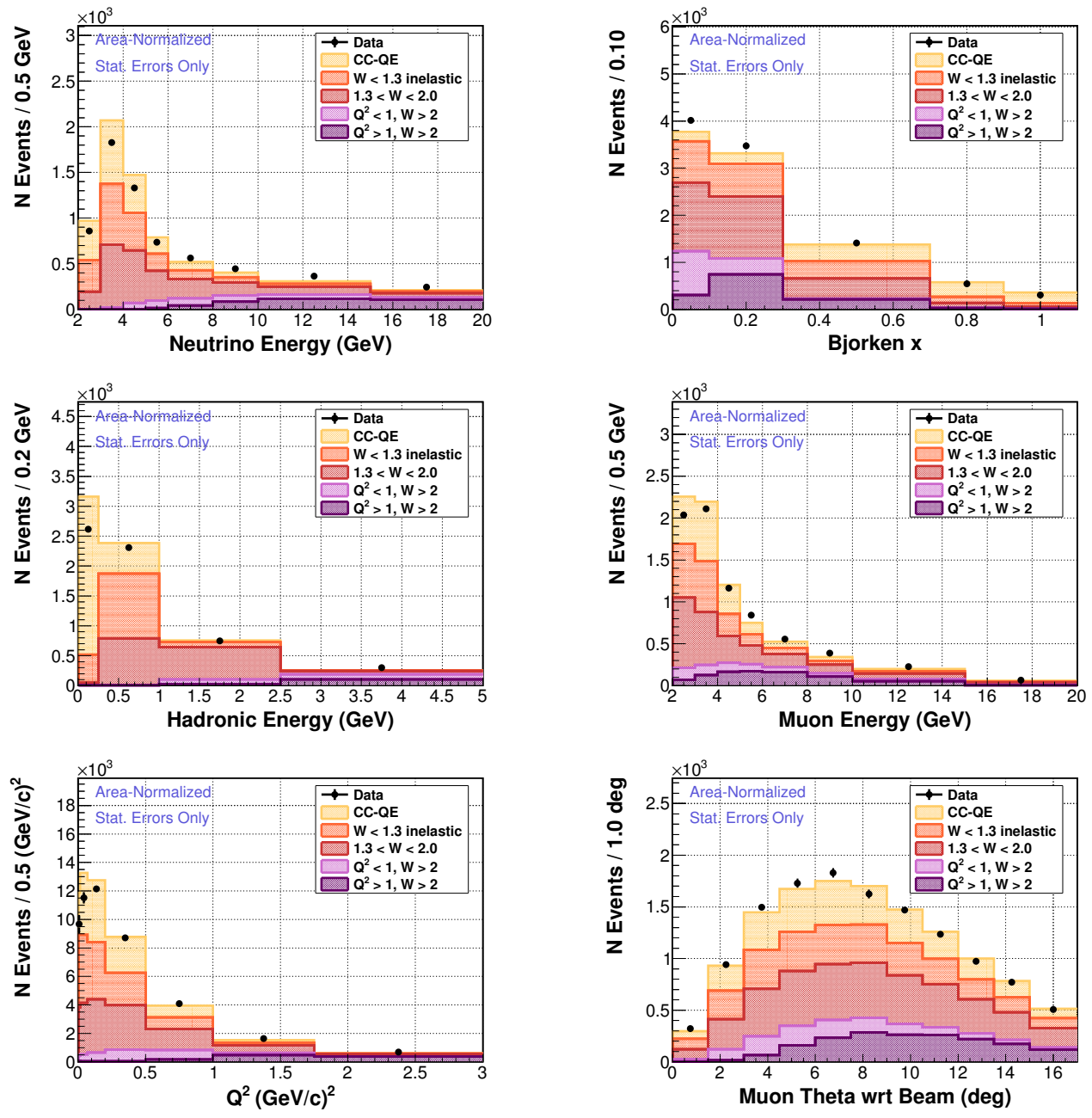

(k) Tracker modules 39-44

Figure A.1: Kinematic distributions of selected events. For explanation, see top of this section. 

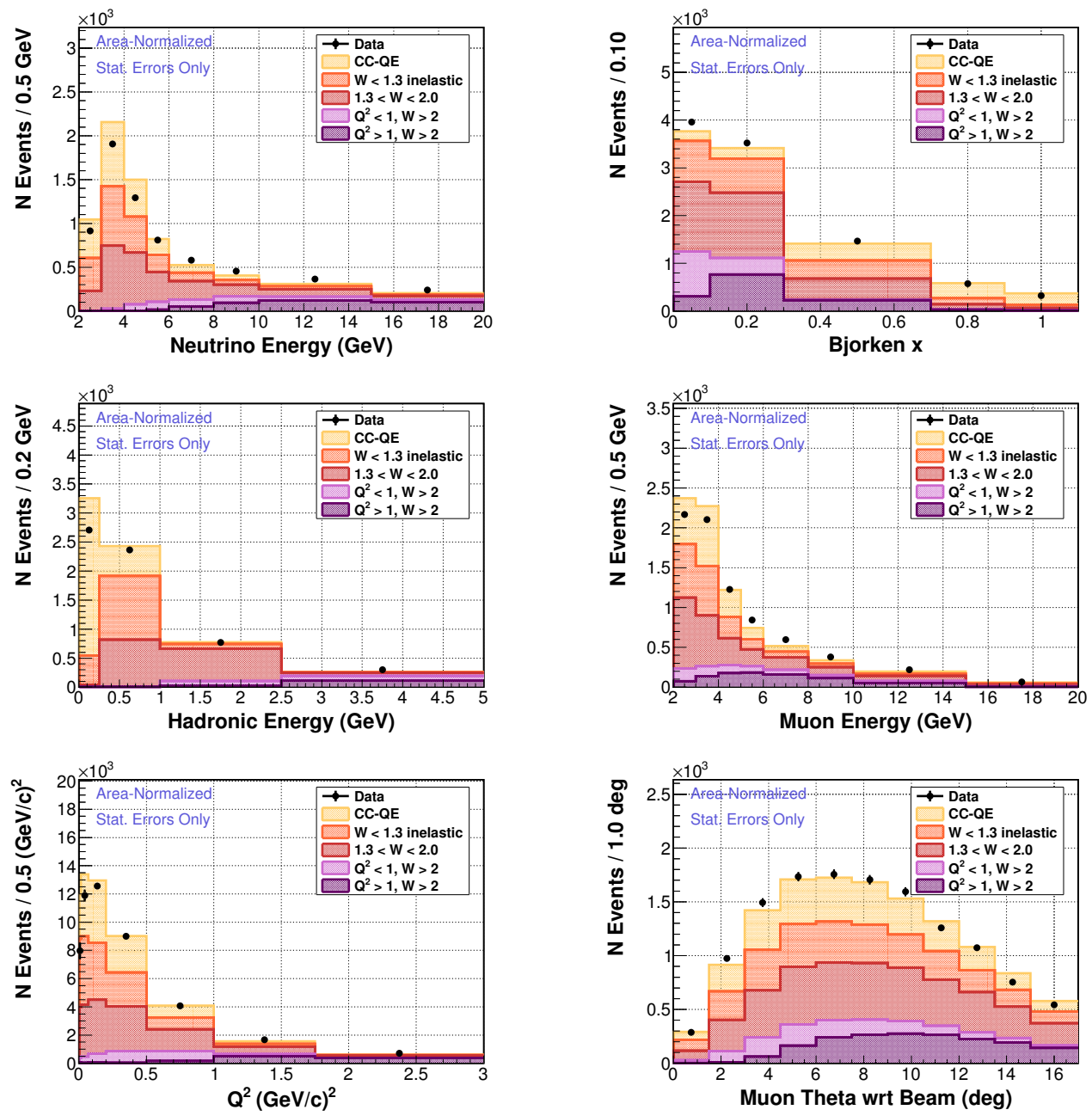

(1) Tracker modules 45-50

Figure A.1: Kinematic distributions of selected events. For explanation, see top of this section. 

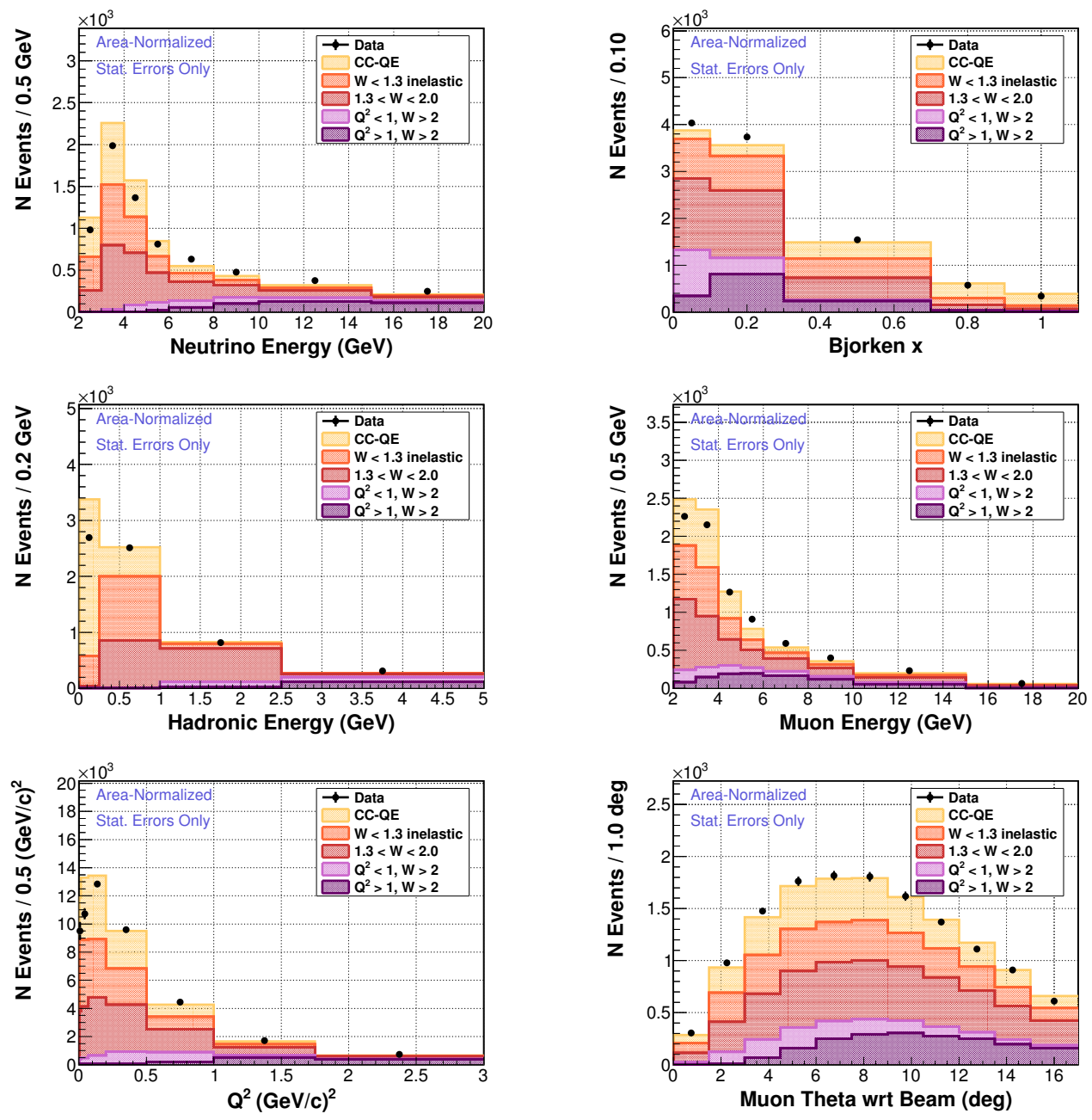

(m) Tracker modules 51-56

Figure A.1: Kinematic distributions of selected events. For explanation, see top of this section. 

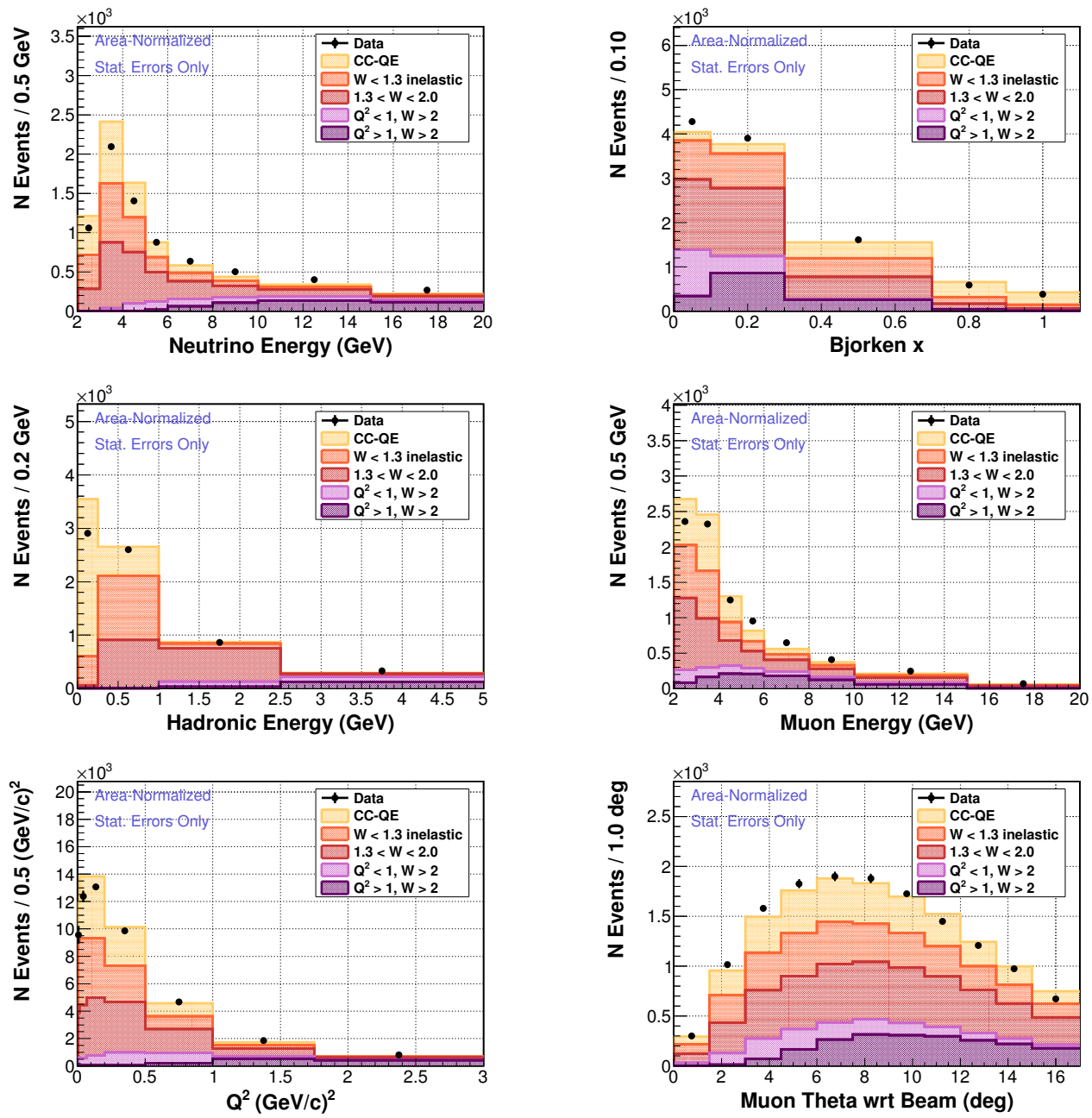

(n) Tracker modules 57-62

Figure A.1: Kinematic distributions of selected events. For explanation, see top of this section. 

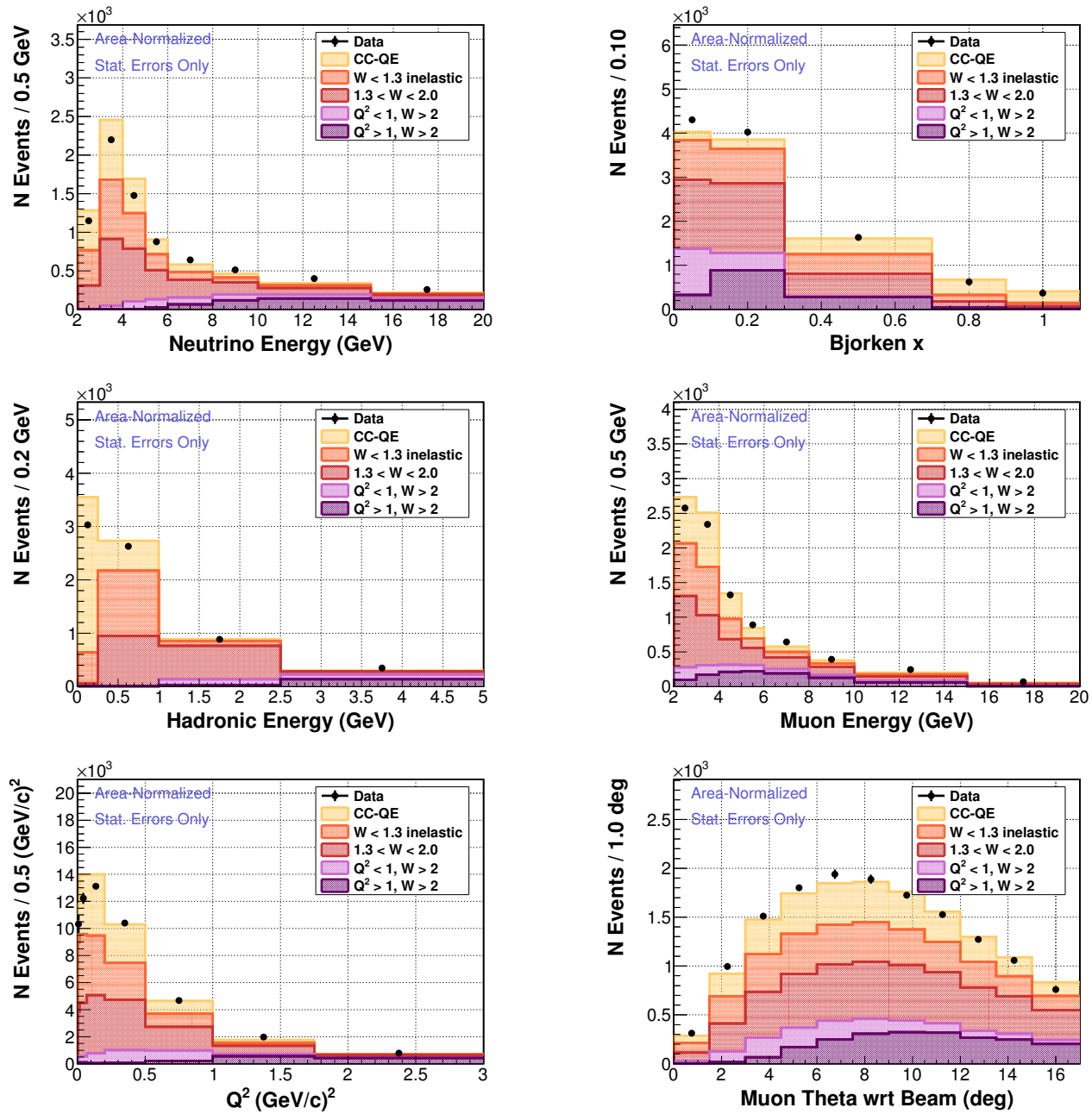

(o) Tracker modules 63-68

Figure A.1: Kinematic distributions of selected events. For explanation, see top of this section. 

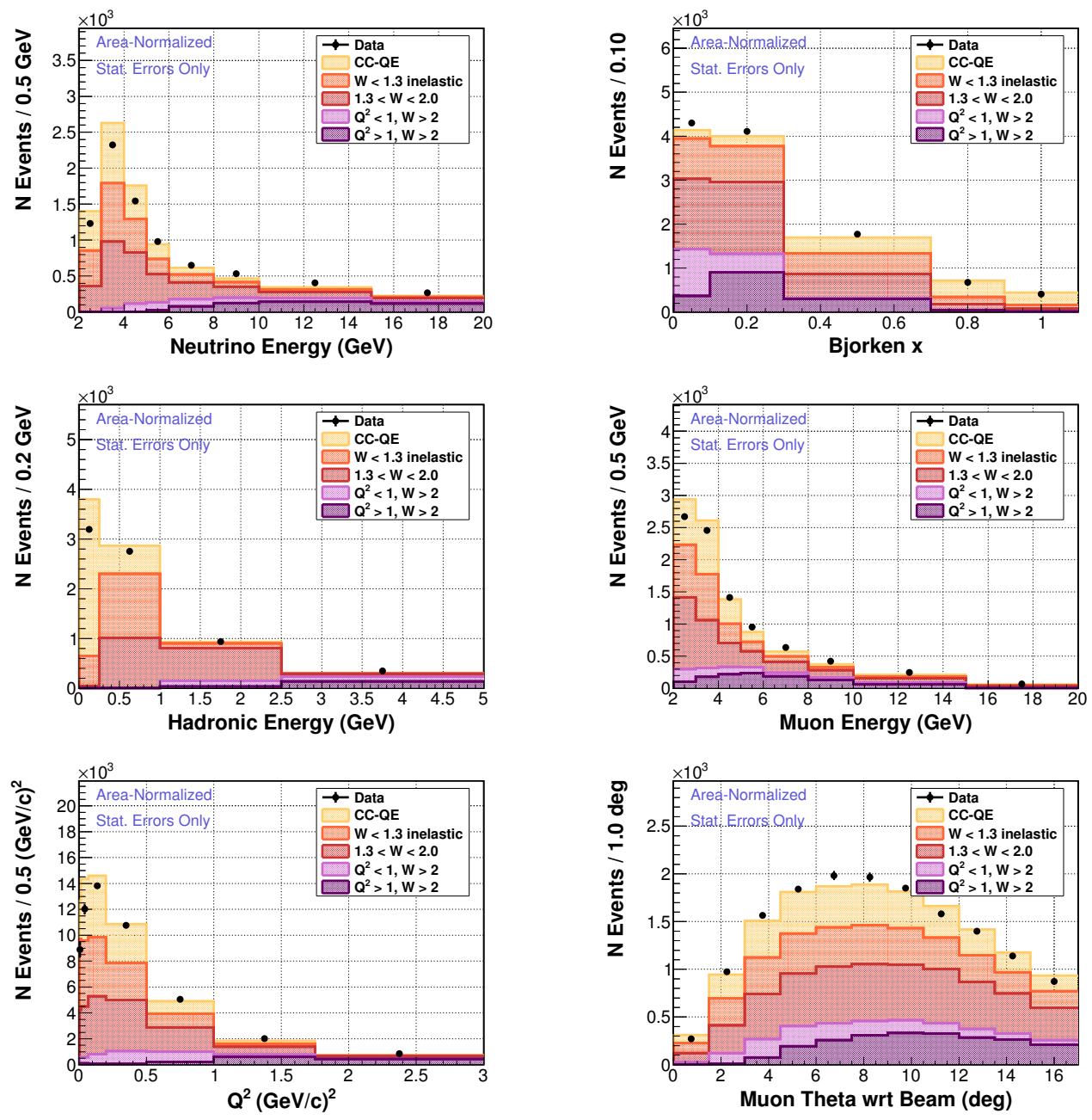

(p) Tracker modules 69-74

Figure A.1: Kinematic distributions of selected events. For explanation, see top of this section. 

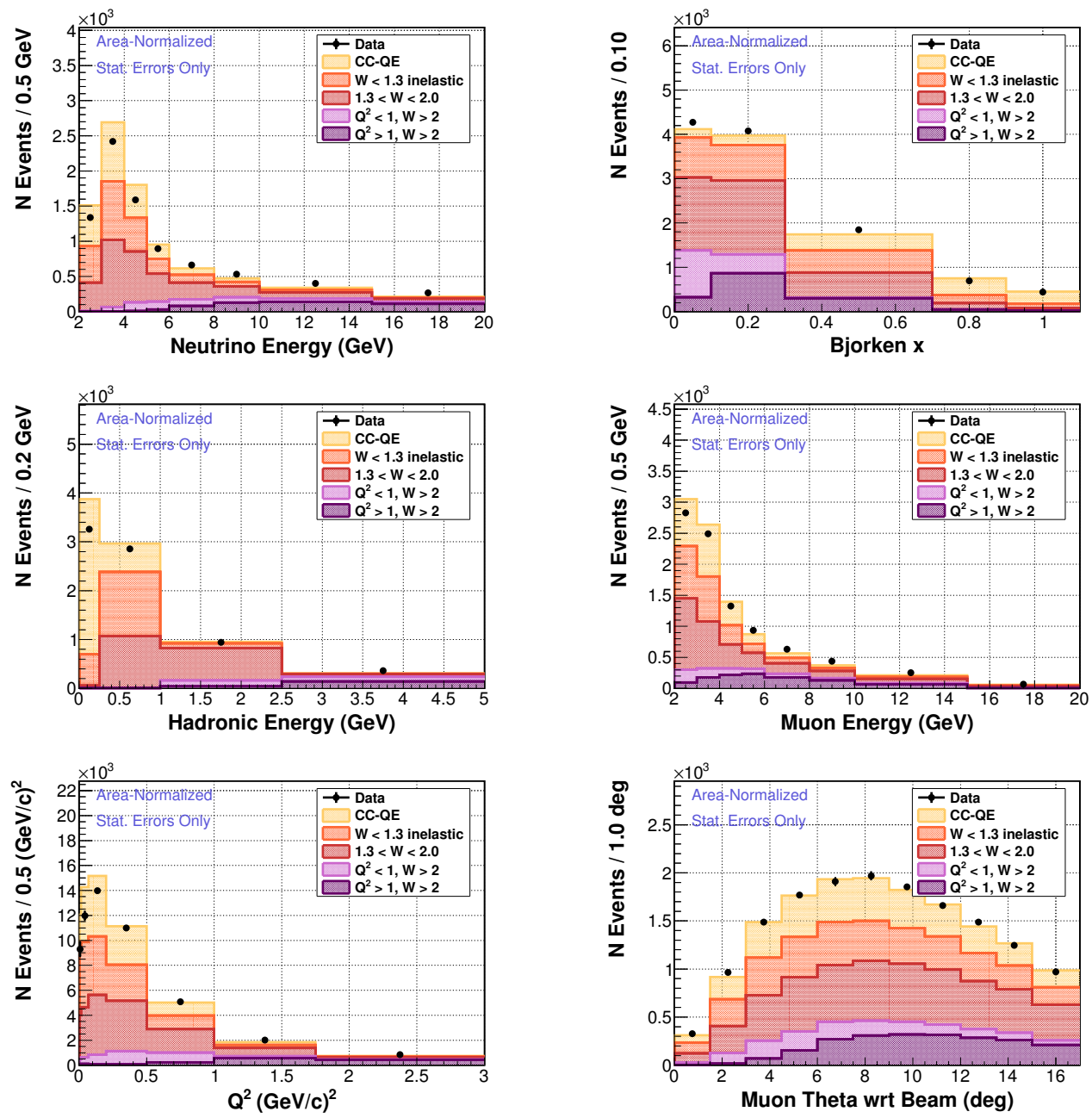

(q) Tracker modules $75-80$

Figure A.1: Kinematic distributions of selected events. For explanation, see top of this section. 


\section{A.2 Predicted Neutral Current and Wrong Sign Background}

Figures in this section show the the fraction of the event samples which are neutral currnent (NC) and wrong sign (WS) background. Wrong sign means the event was $\bar{\nu}_{\mu}$ instead of $\nu_{\mu}$. $\mathrm{NC}$ is shown as blue circles, and WS as green squares.
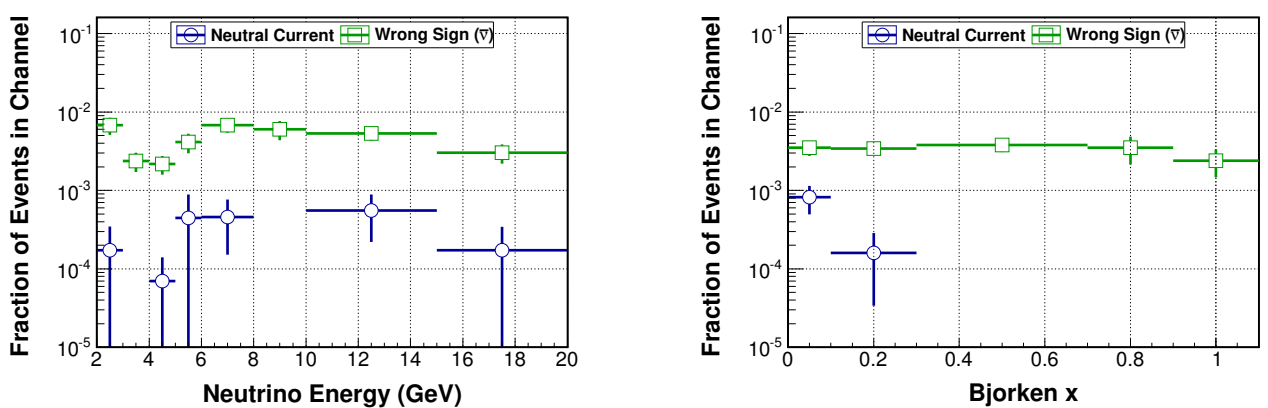

(a) Target 2 iron

Figure A.2: Predicted neutral current and wrong sign backgrounds. For explanation, see top of this section.
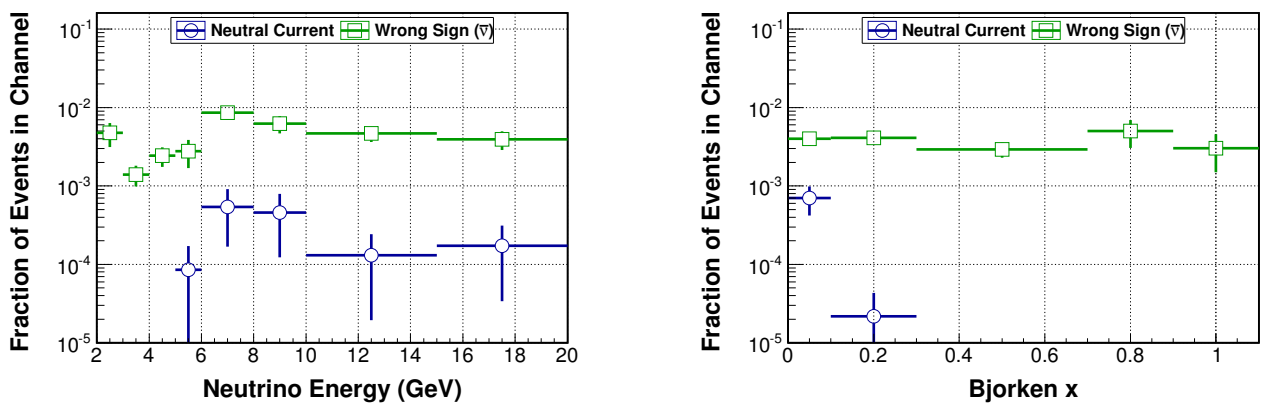

(b) Target 2 lead

Figure A.2: Predicted neutral current and wrong sign backgrounds. For explanation, see top of this section. 

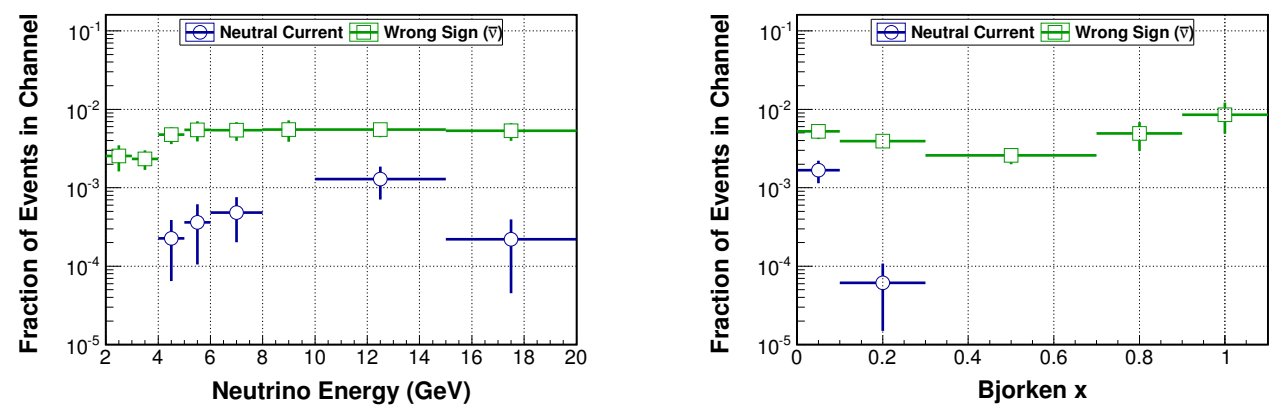

(c) Target 3 carbon

Figure A.2: Predicted neutral current and wrong sign backgrounds. For explanation, see top of this section.
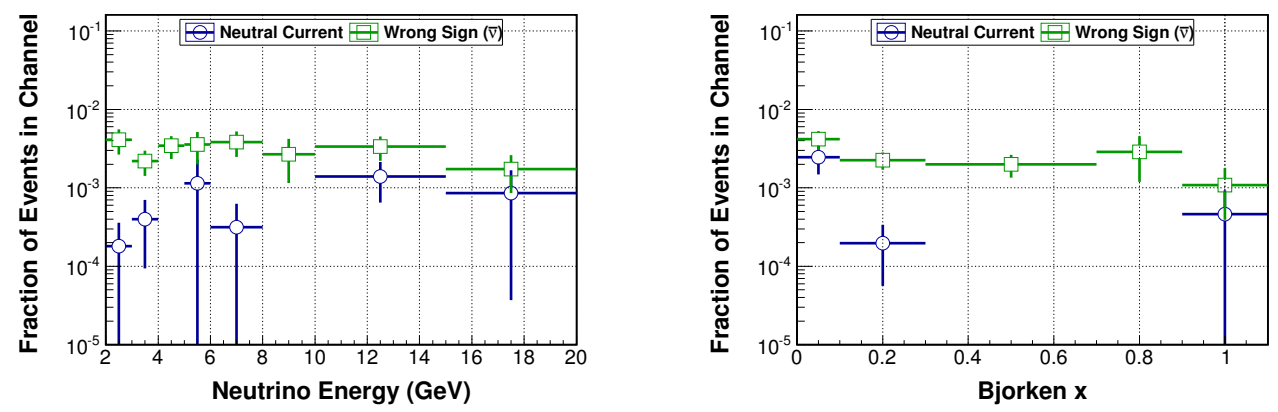

(d) Target 3 iron

Figure A.2: Predicted neutral current and wrong sign backgrounds. For explanation, see top of this section.
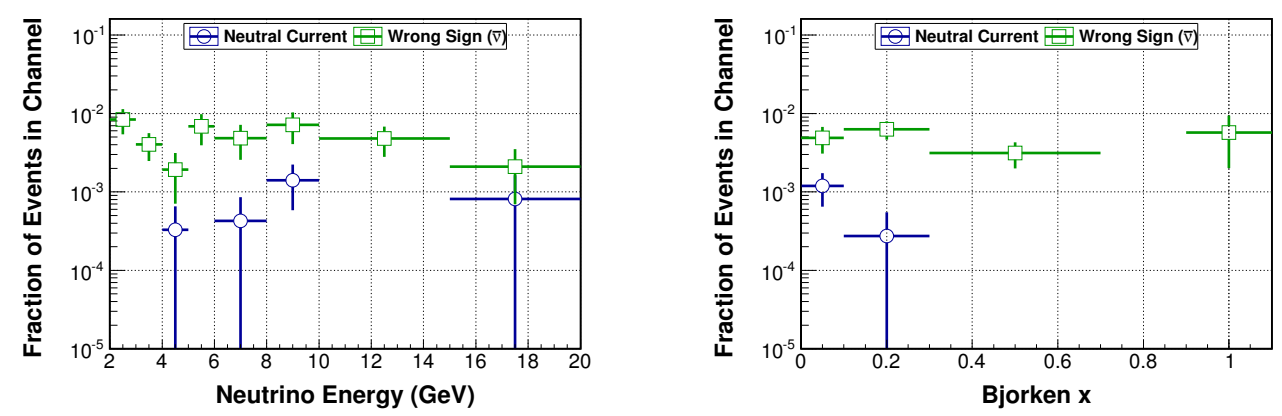

(e) Target 3 lead

Figure A.2: Predicted neutral current and wrong sign backgrounds. For explanation, see top of this section. 

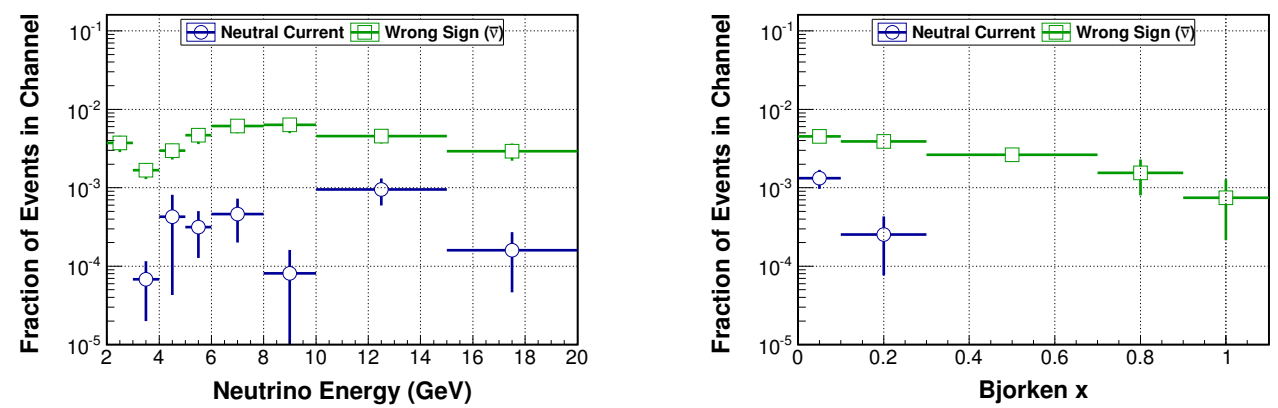

(f) Target 4 lead

Figure A.2: Predicted neutral current and wrong sign backgrounds. For explanation, see top of this section.
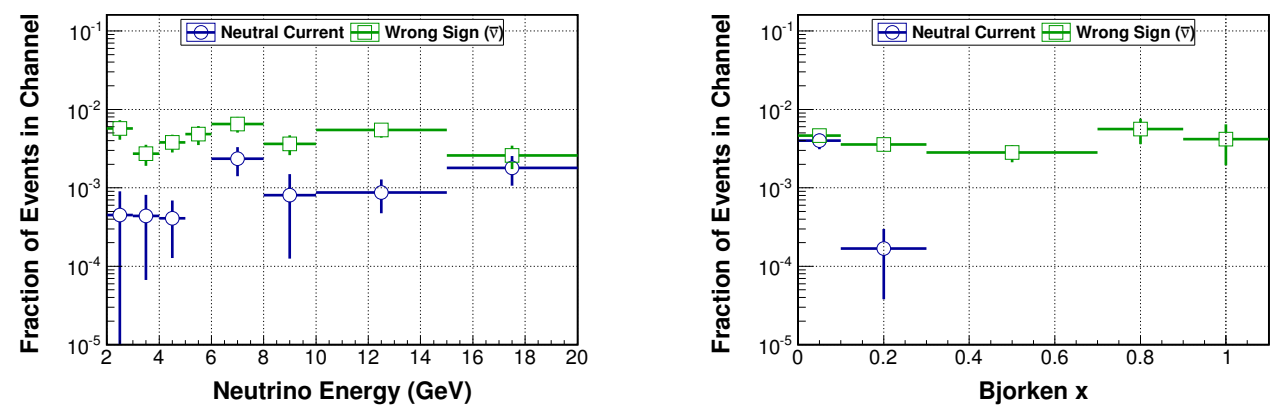

(g) Target 5 iron

Figure A.2: Predicted neutral current and wrong sign backgrounds. For explanation, see top of this section.
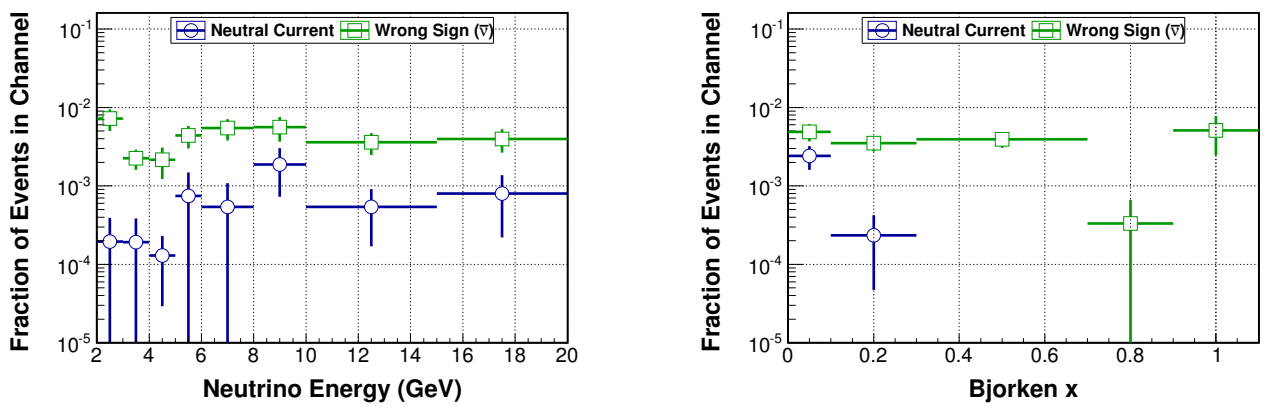

(h) Target 5 lead

Figure A.2: Predicted neutral current and wrong sign backgrounds. For explanation, see top of this section. 

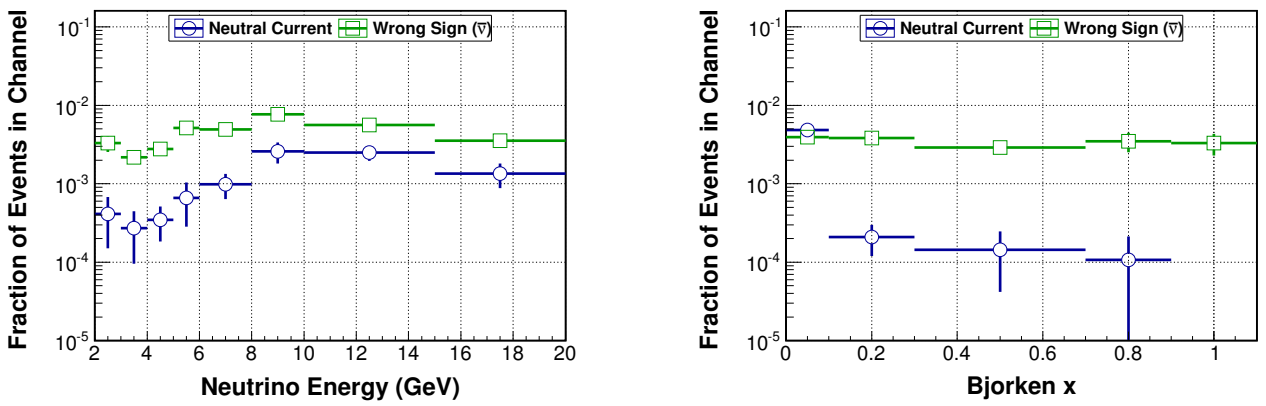

(i) Tracker modules 27-32

Figure A.2: Predicted neutral current and wrong sign backgrounds. For explanation, see top of this section.
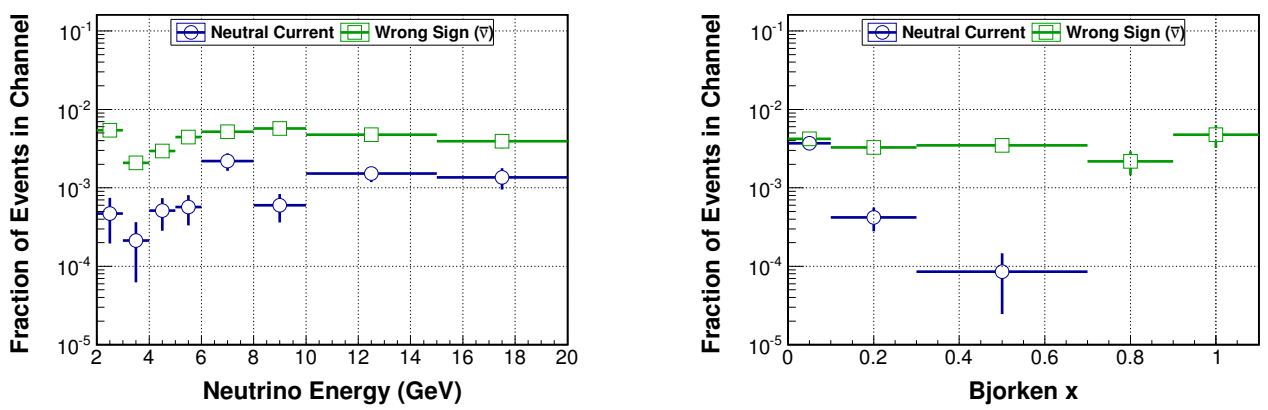

(j) Tracker modules 33-38

Figure A.2: Predicted neutral current and wrong sign backgrounds. For explanation, see top of this section.
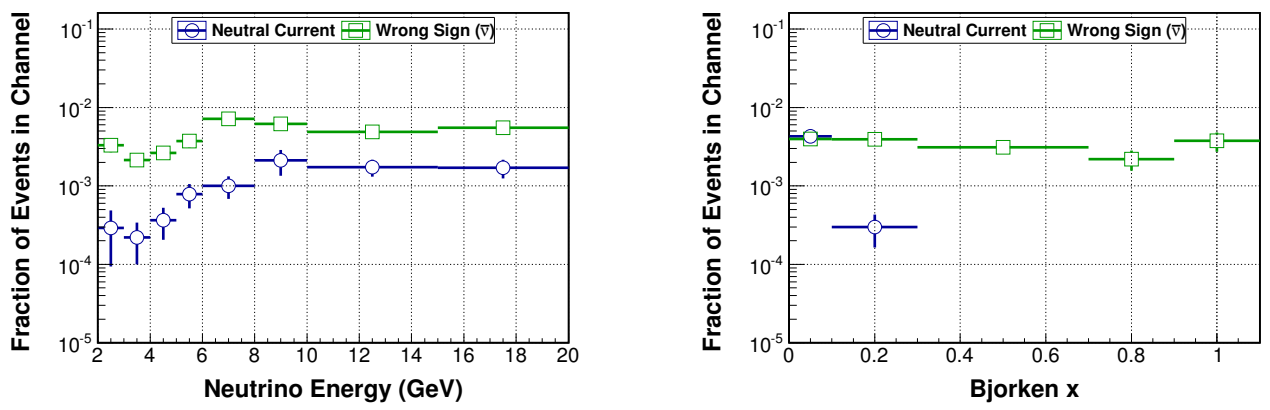

(k) Tracker modules 39-44

Figure A.2: Predicted neutral current and wrong sign backgrounds. For explanation, see top of this section. 

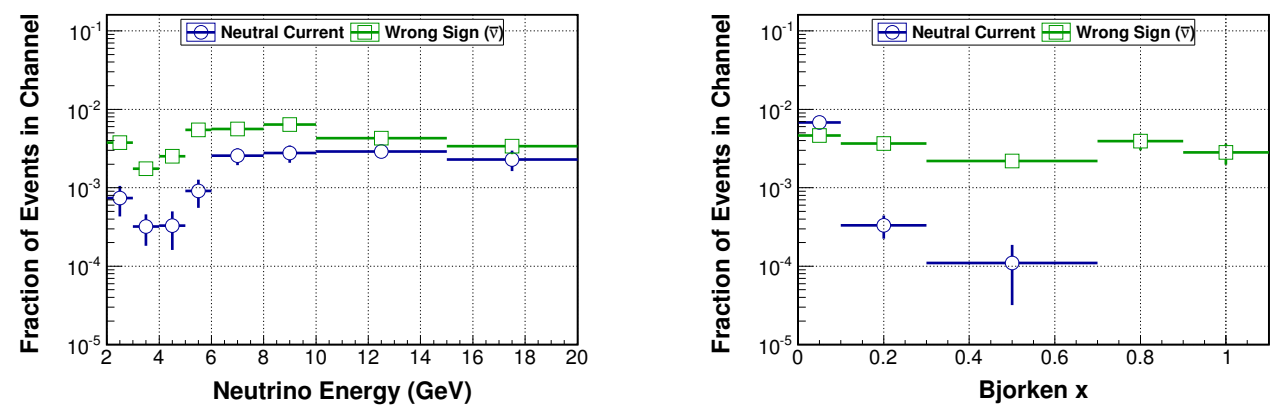

(1) Tracker modules 45-50

Figure A.2: Predicted neutral current and wrong sign backgrounds. For explanation, see top of this section.
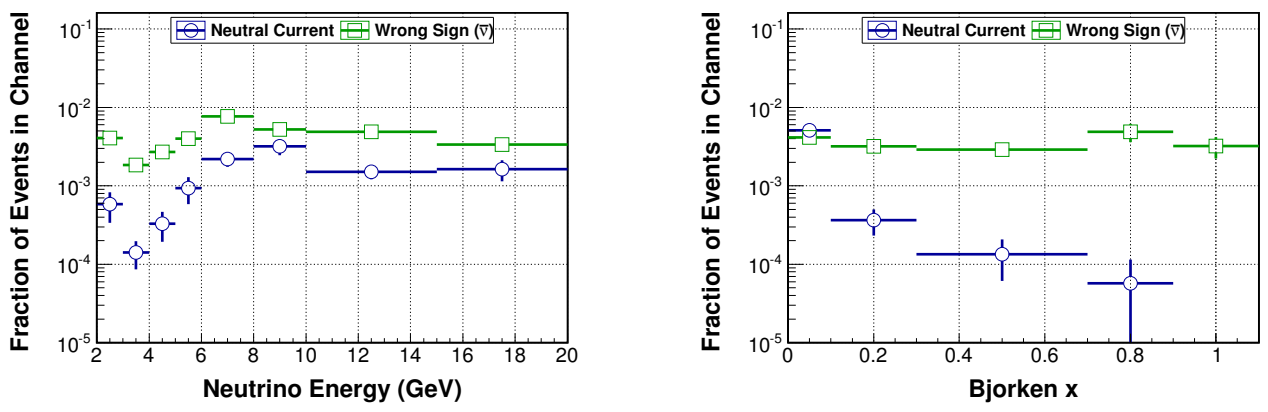

(m) Tracker modules 51-56

Figure A.2: Predicted neutral current and wrong sign backgrounds. For explanation, see top of this section.
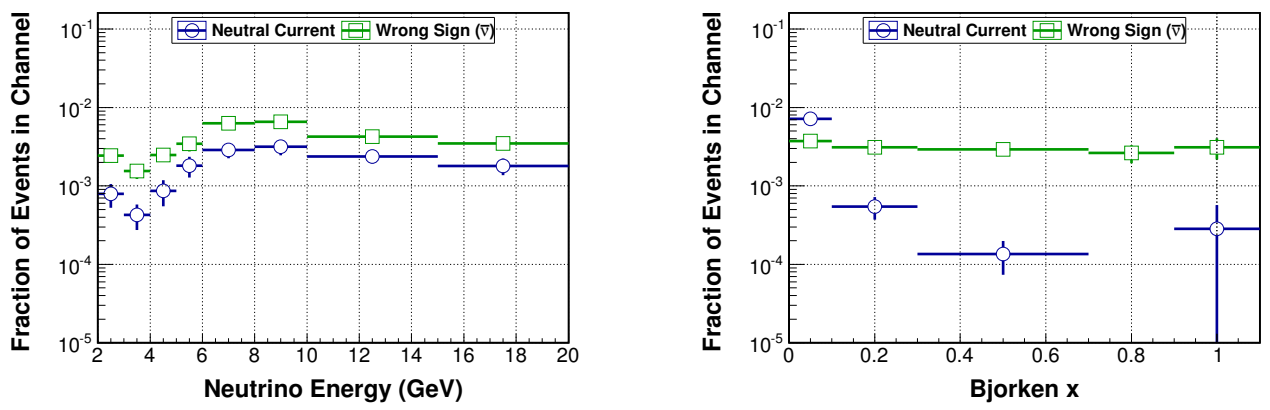

(n) Tracker modules 57-62

Figure A.2: Predicted neutral current and wrong sign backgrounds. For explanation, see top of this section. 

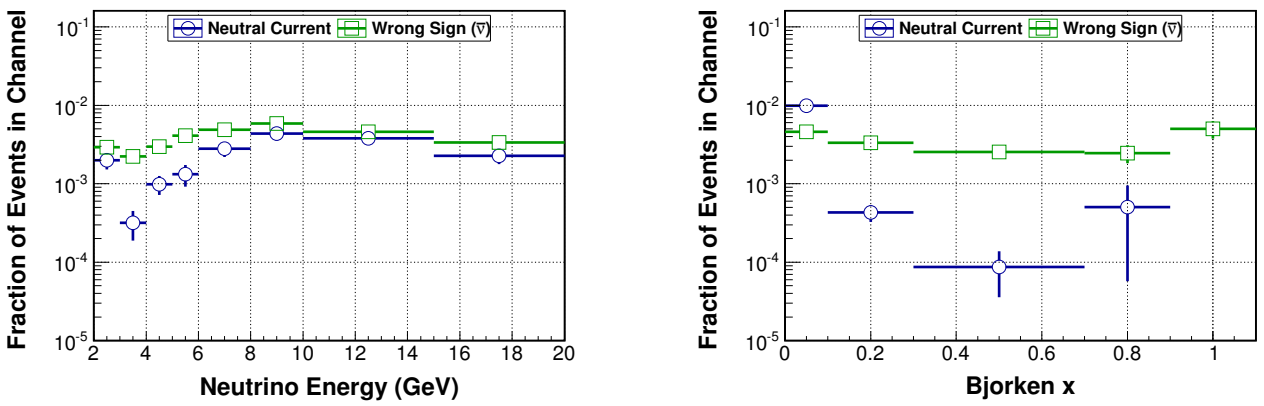

(o) Tracker modules 63-68

Figure A.2: Predicted neutral current and wrong sign backgrounds. For explanation, see top of this section.
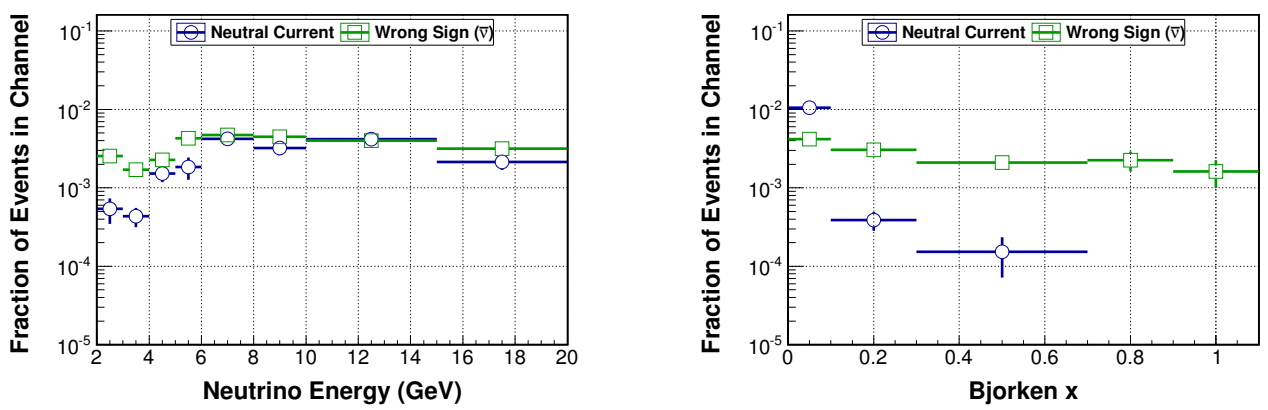

(p) Tracker modules 69-74

Figure A.2: Predicted neutral current and wrong sign backgrounds. For explanation, see top of this section.
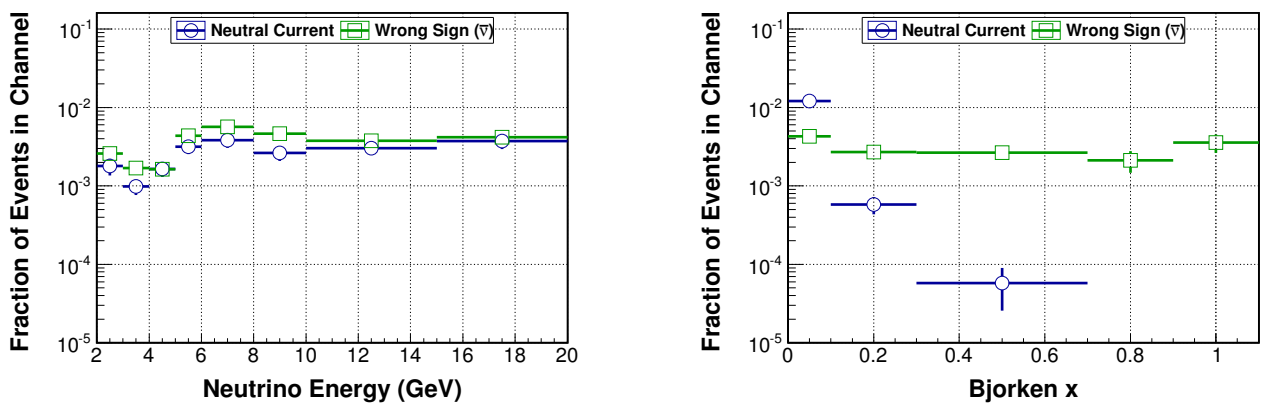

(q) Tracker modules $75-80$

Figure A.2: Predicted neutral current and wrong sign backgrounds. For explanation, see top of this section. 


\section{A.3 Event Sample Vertex Distributions}

The figures in this section show:

- Top - Distributions of reconstructed vertex positions $\mathrm{x}$ and $\mathrm{y}$.

- Middle - Ratio of data to simulation for vertex distributions.

- Bottom - Fraction of sample that is contamination from scintillator. Shown for passive target only.

The simulation (MC) histograms are colored by the vertex material in which the event was generated for the passive target event samples.

- Carbon - Green

- Iron - Red

- Lead - Blue

- Upstream Scintillator - Orange - The generated vertex is in scintillator upstream of the reconstructed vertex.

- Downstream Scintillator - Purple - The generated vertex is in scintillator downstream of the reconstructed vertex.

Each page shows a different event sample. 

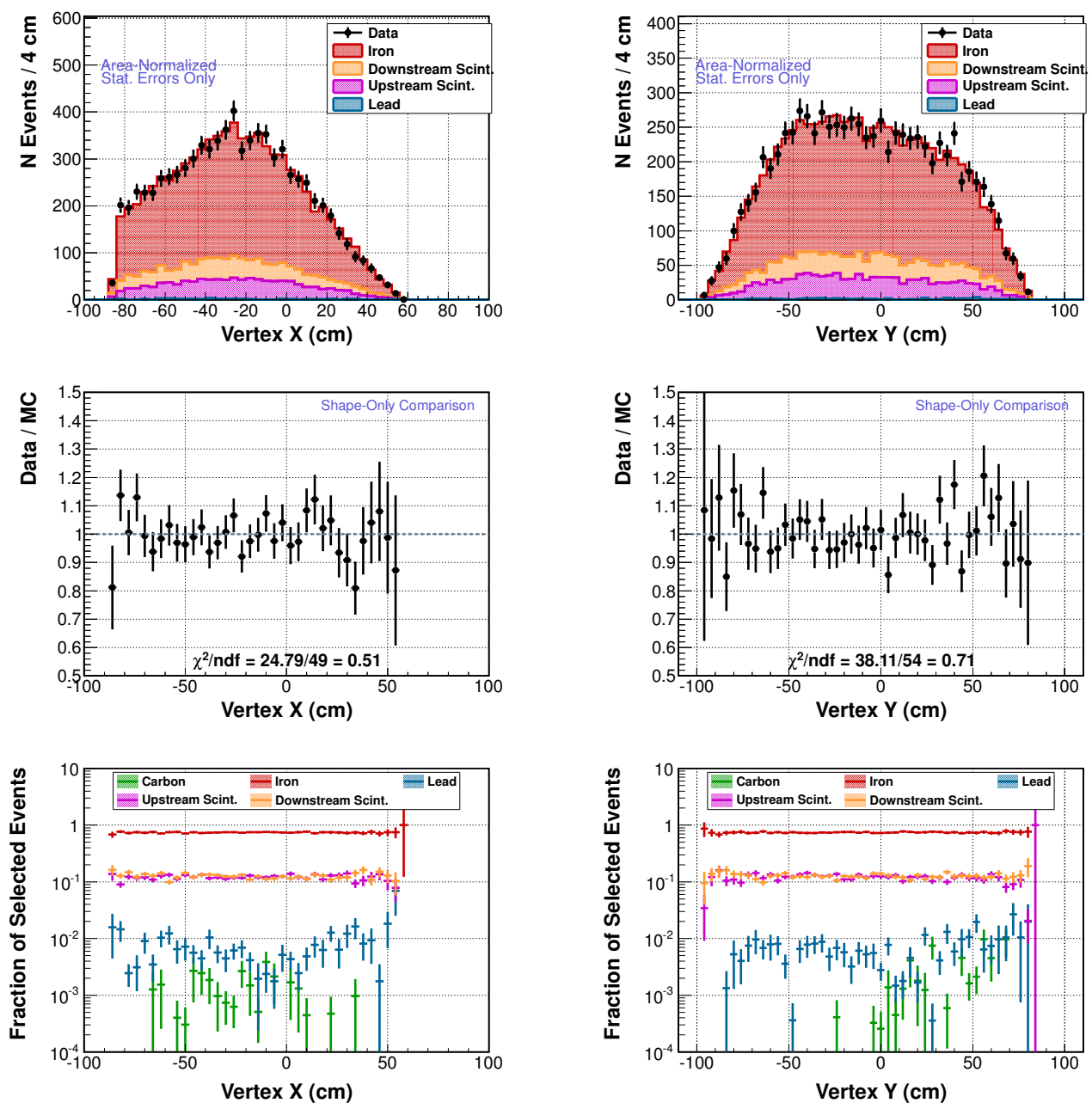

(a) Target 2 iron

Figure A.3: Vertex distributions of selected events. For explanation, see top of this section. 

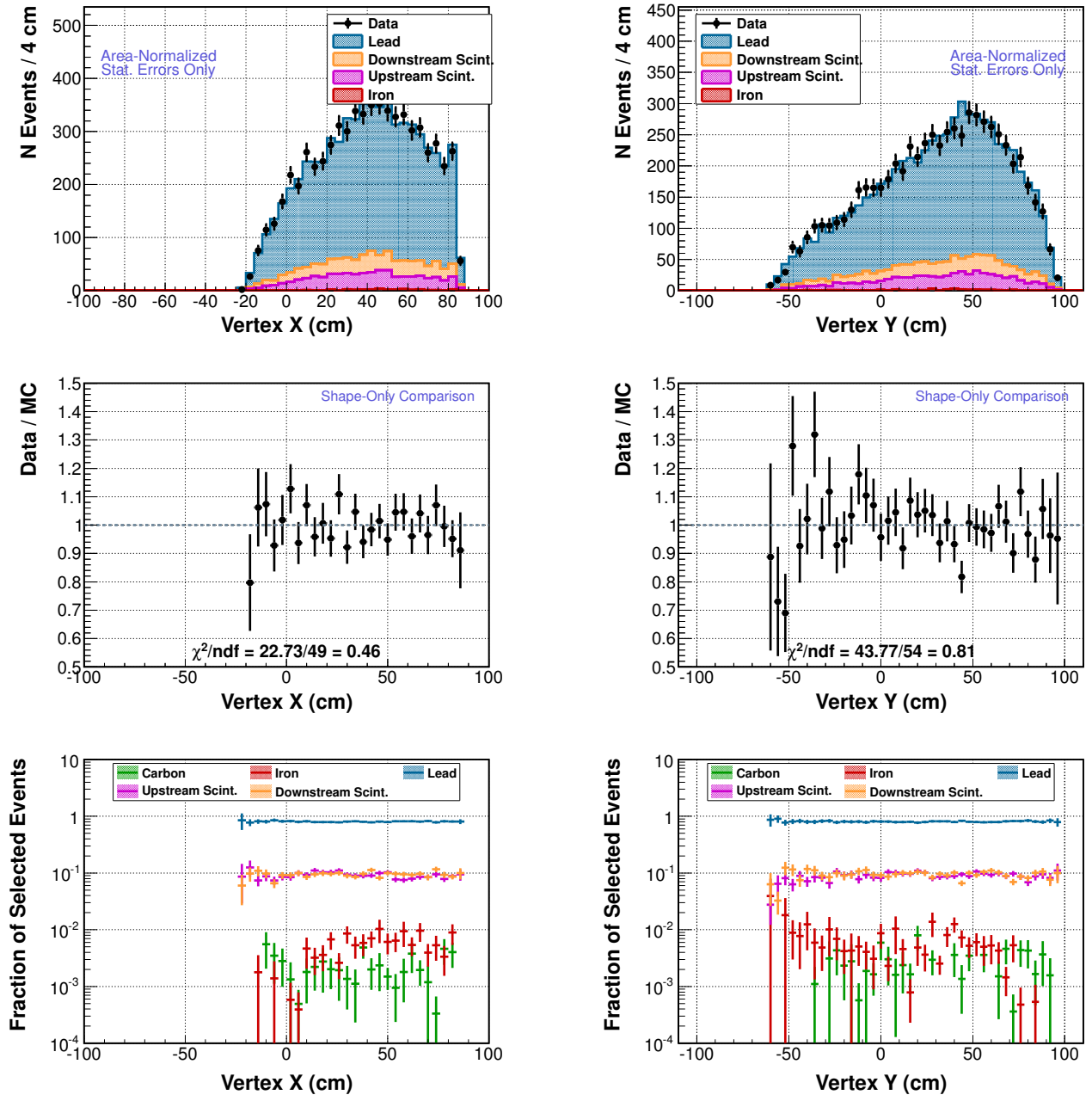

(b) Target 2 lead

Figure A.3: Vertex distributions of selected events. For explanation, see top of this section. 

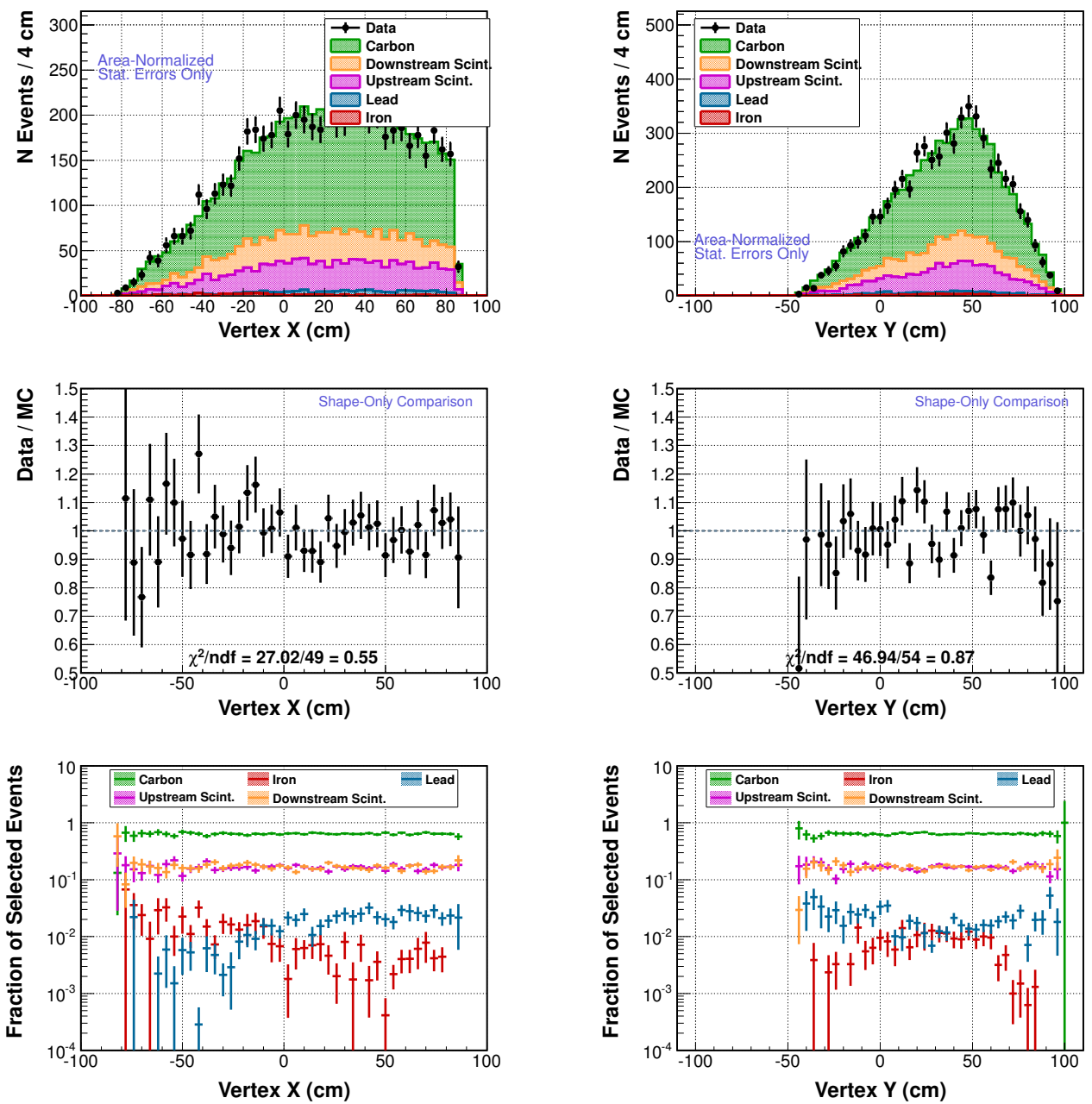

(c) Target 3 carbon

Figure A.3: Vertex distributions of selected events. For explanation, see top of this section. 

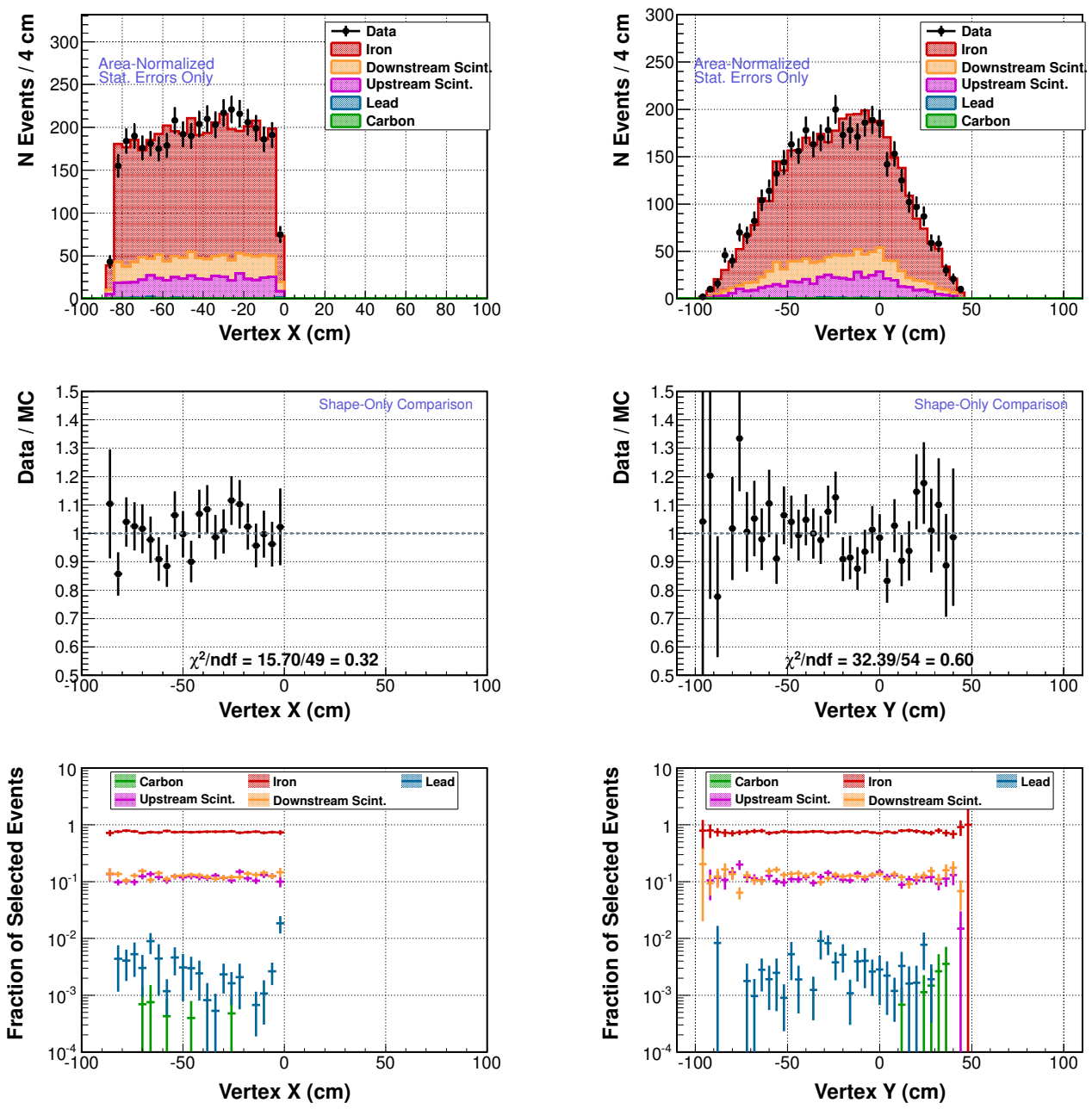

(d) Target 3 iron

Figure A.3: Vertex distributions of selected events. For explanation, see top of this section. 

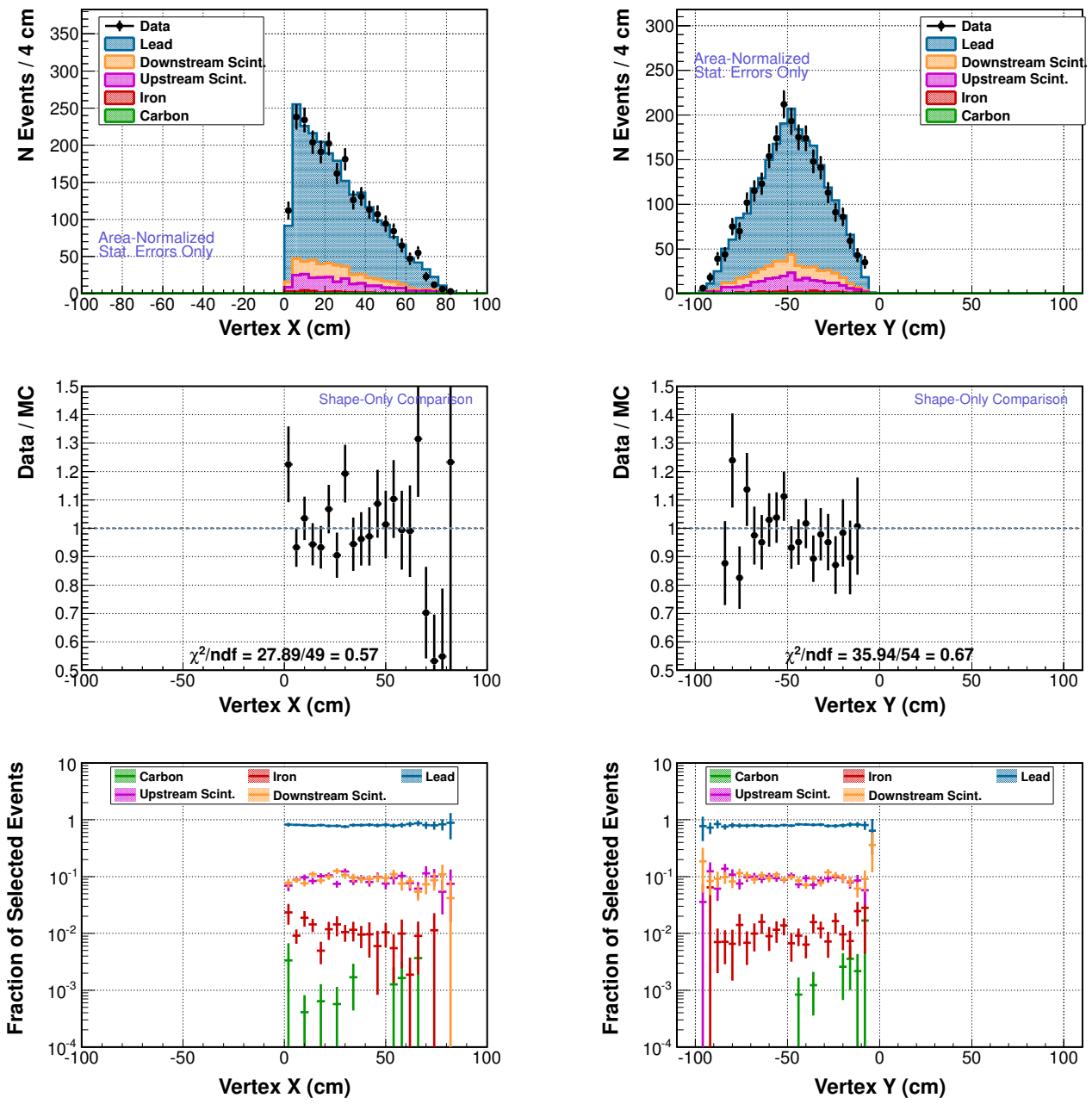

(e) Target 3 lead

Figure A.3: Vertex distributions of selected events. For explanation, see top of this section. 

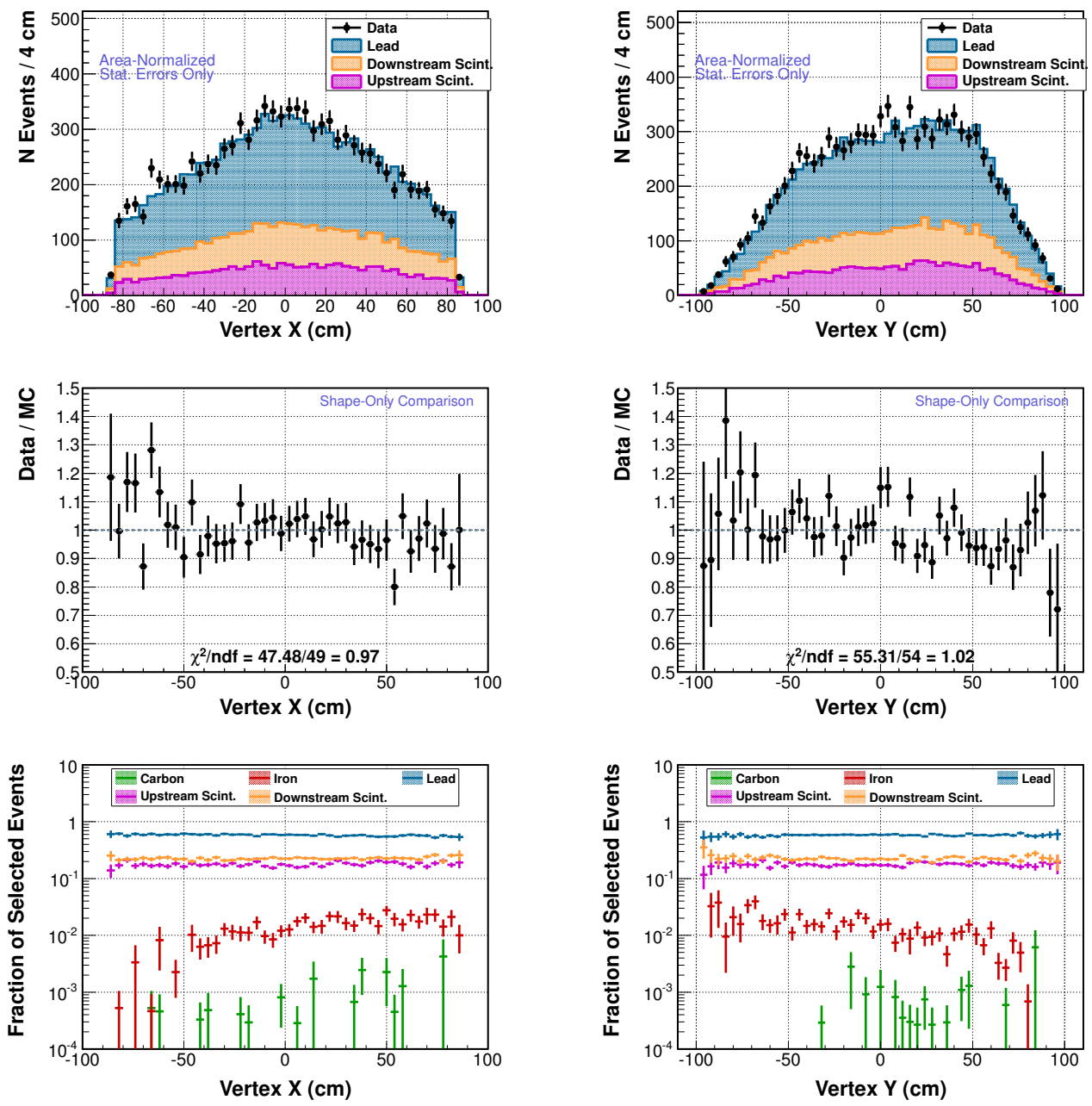

(f) Target 4 lead

Figure A.3: Vertex distributions of selected events. For explanation, see top of this section. 

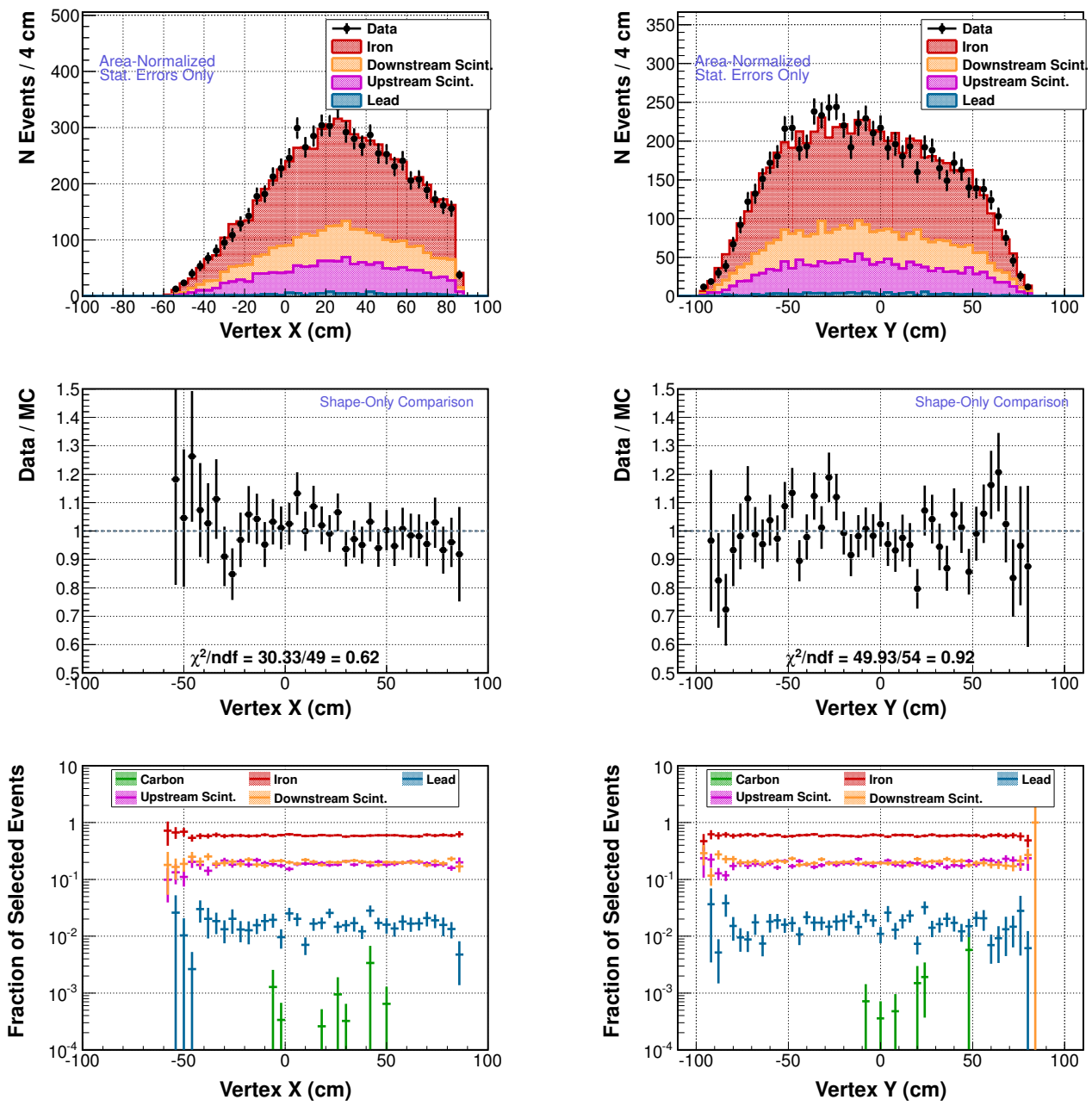

(g) Target 5 iron

Figure A.3: Vertex distributions of selected events. For explanation, see top of this section. 

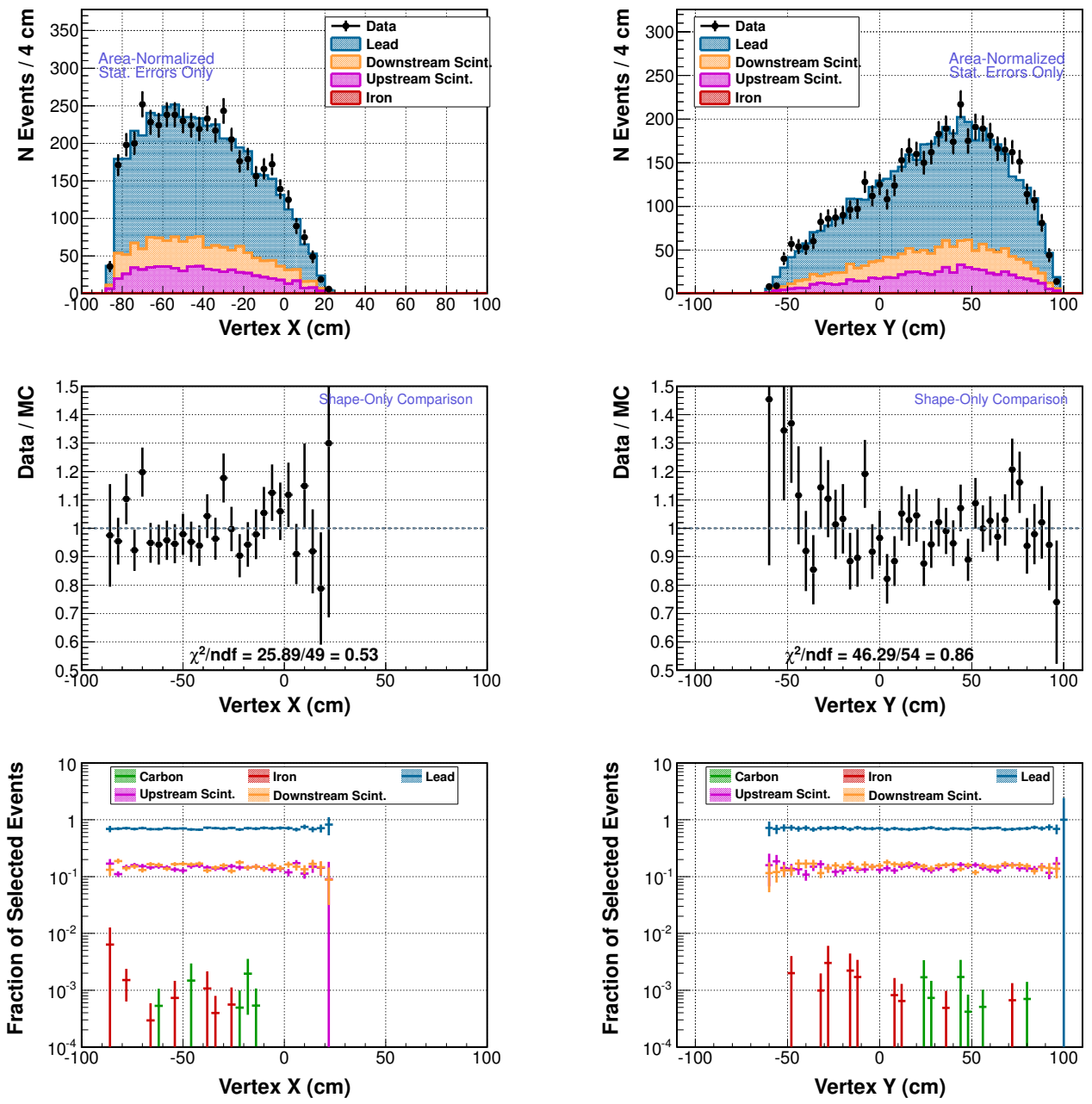

(h) Target 5 lead

Figure A.3: Vertex distributions of selected events. For explanation, see top of this section. 

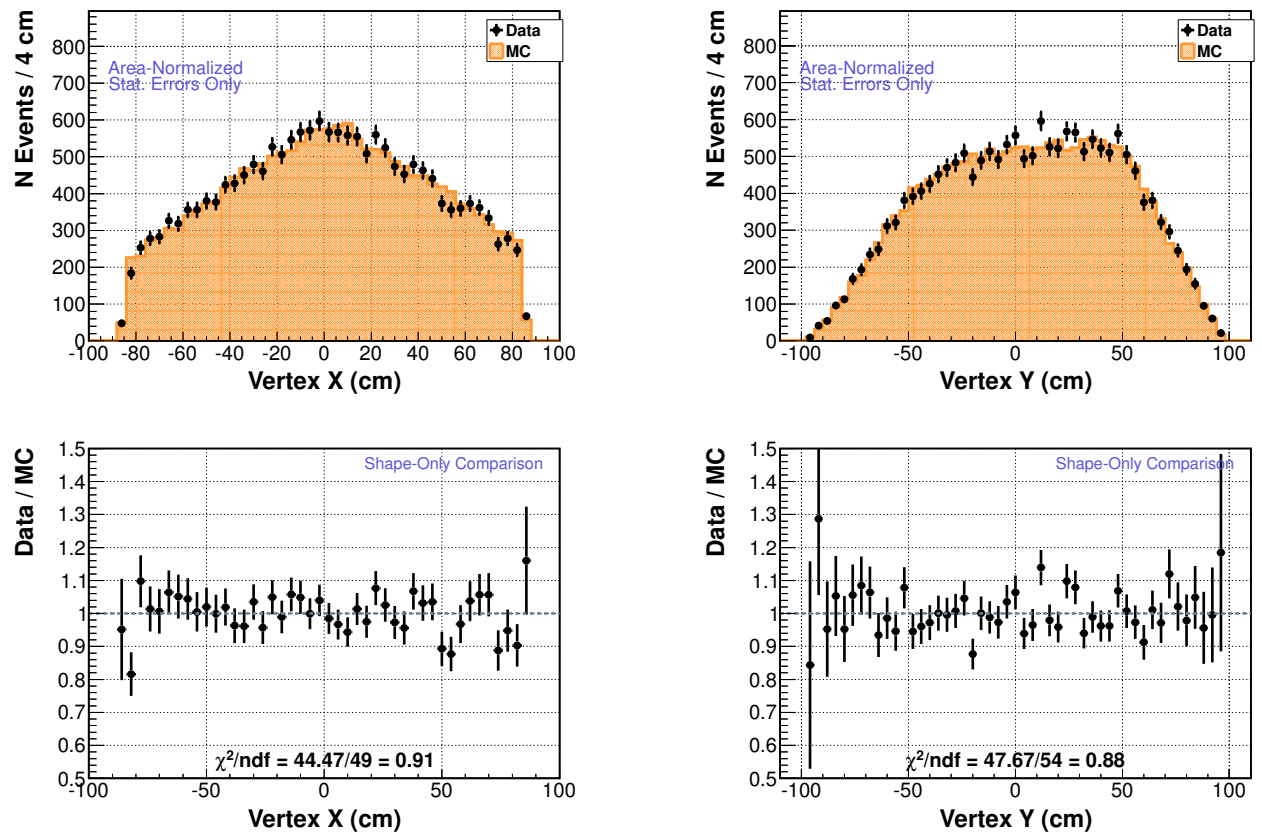

Figure A.3: Vertex distributions of selected events. For explanation, see top of this section. 

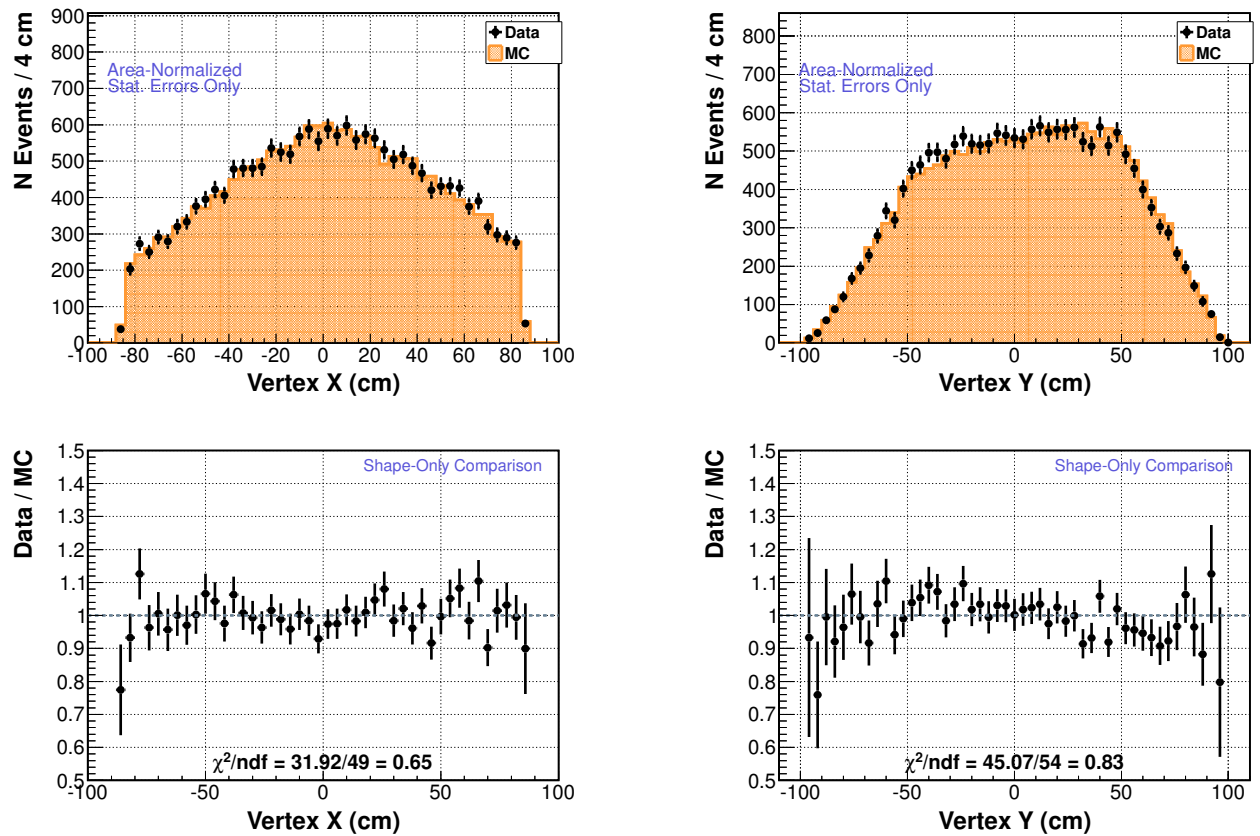

Figure A.3: Vertex distributions of selected events. For explanation, see top of this section. 

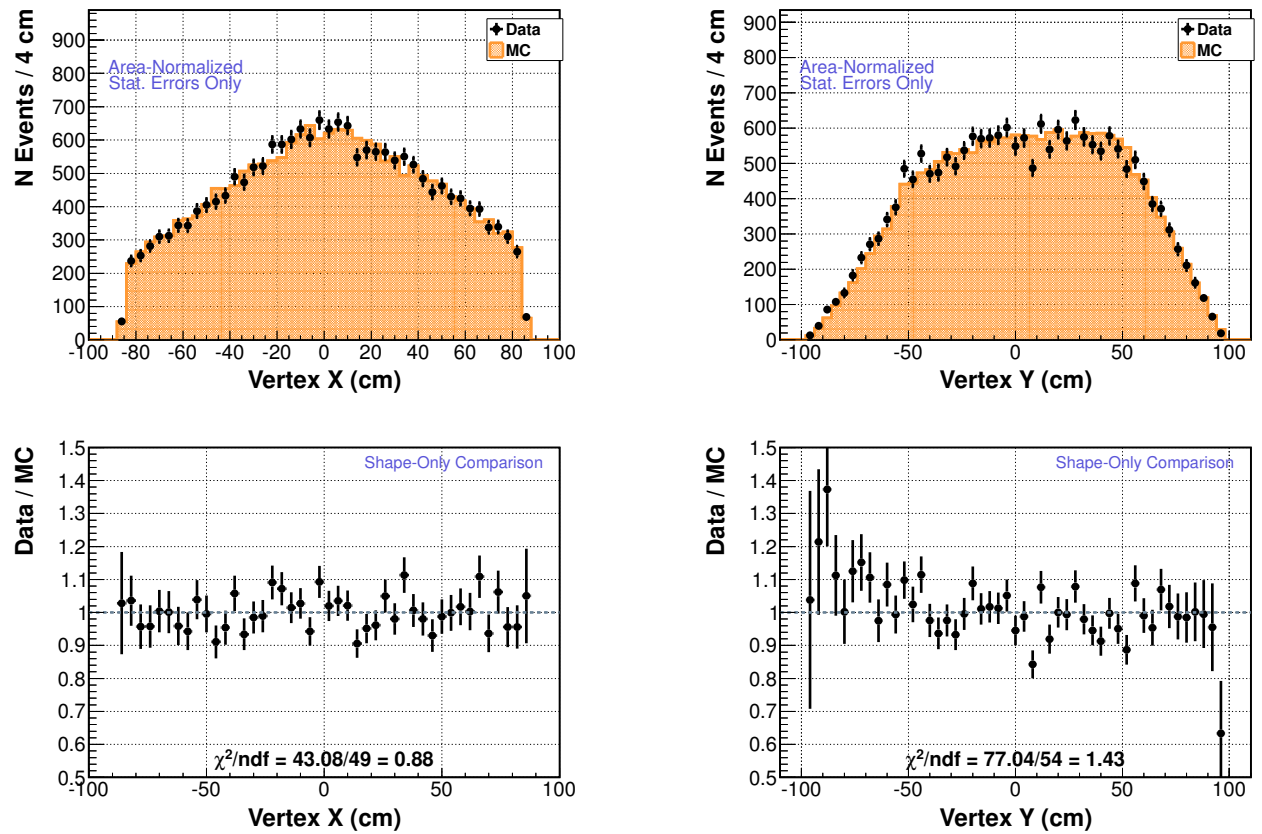

Figure A.3: Vertex distributions of selected events. For explanation, see top of this section. 

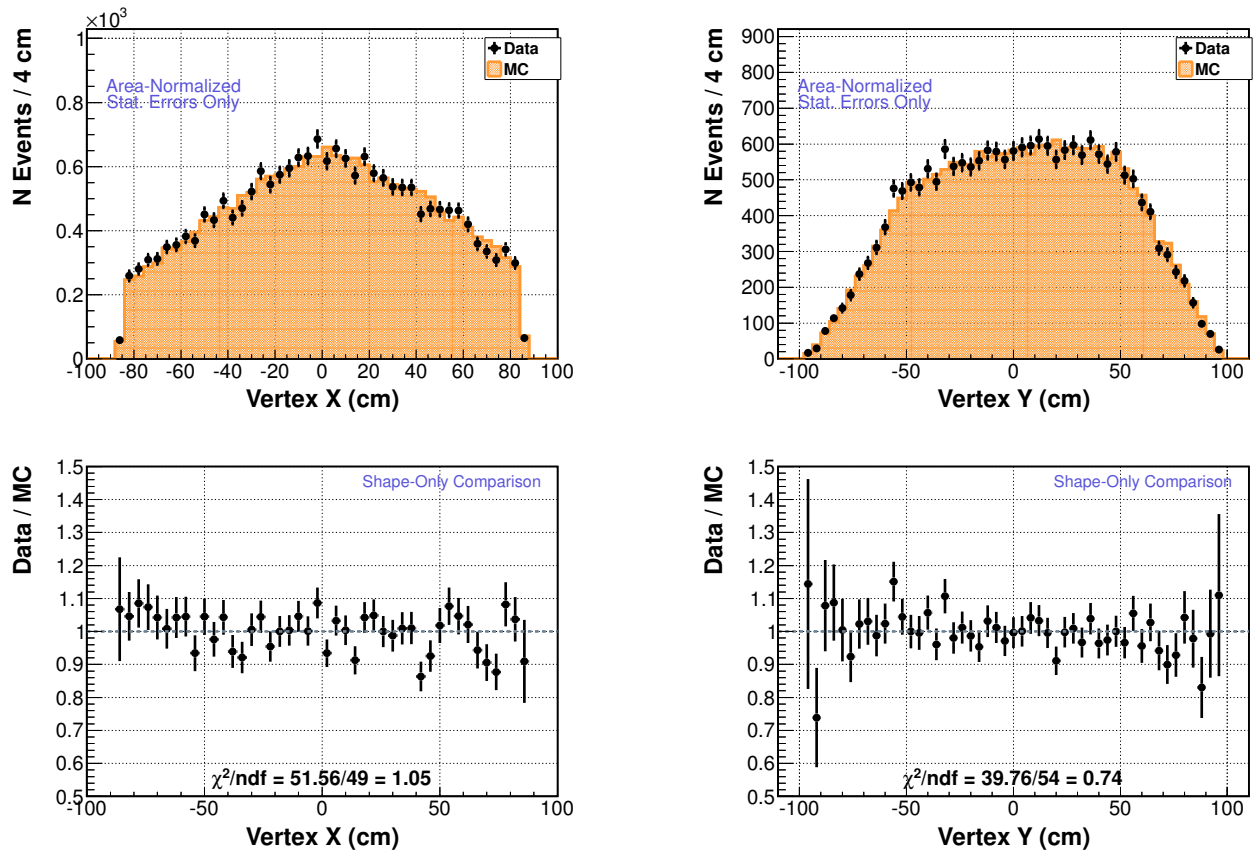

Figure A.3: Vertex distributions of selected events. For explanation, see top of this section. 

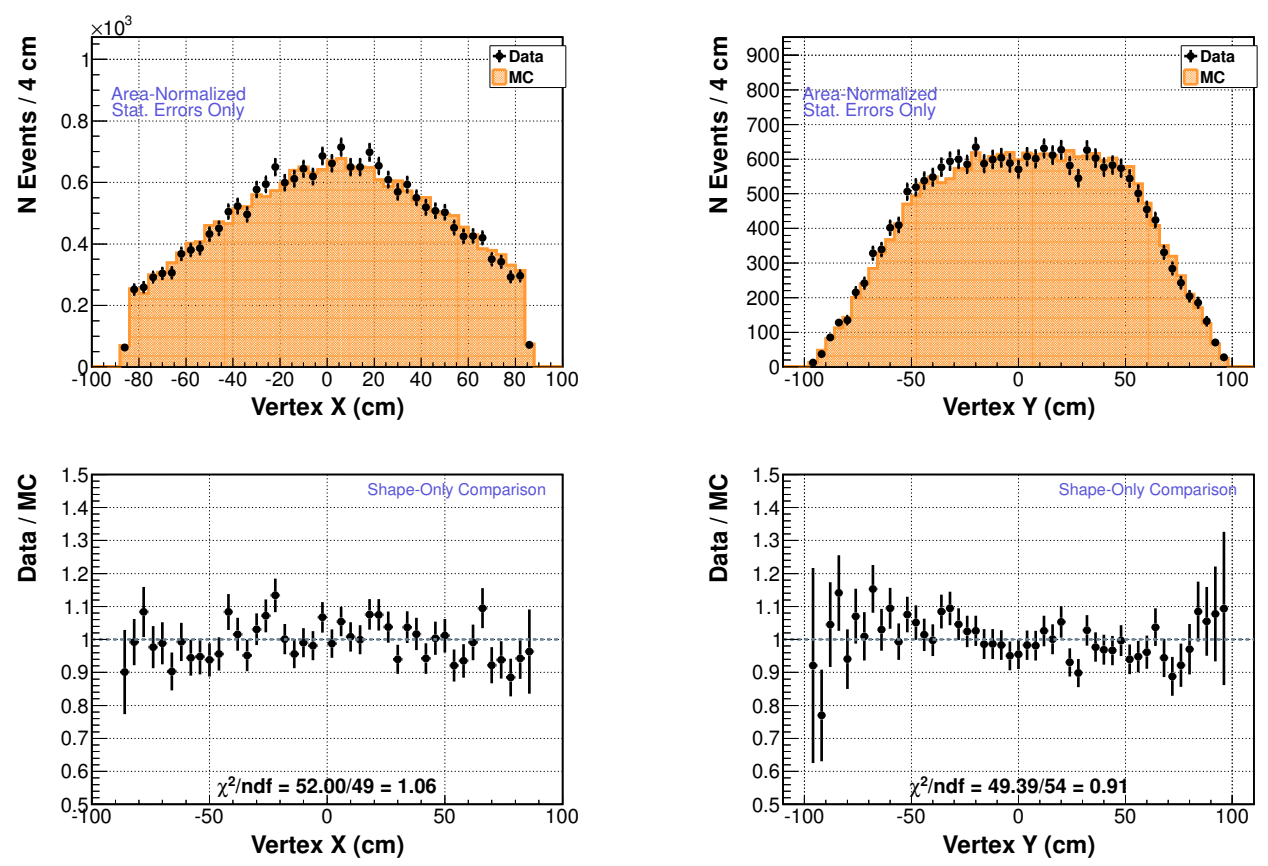

Figure A.3: Vertex distributions of selected events. For explanation, see top of this section. 

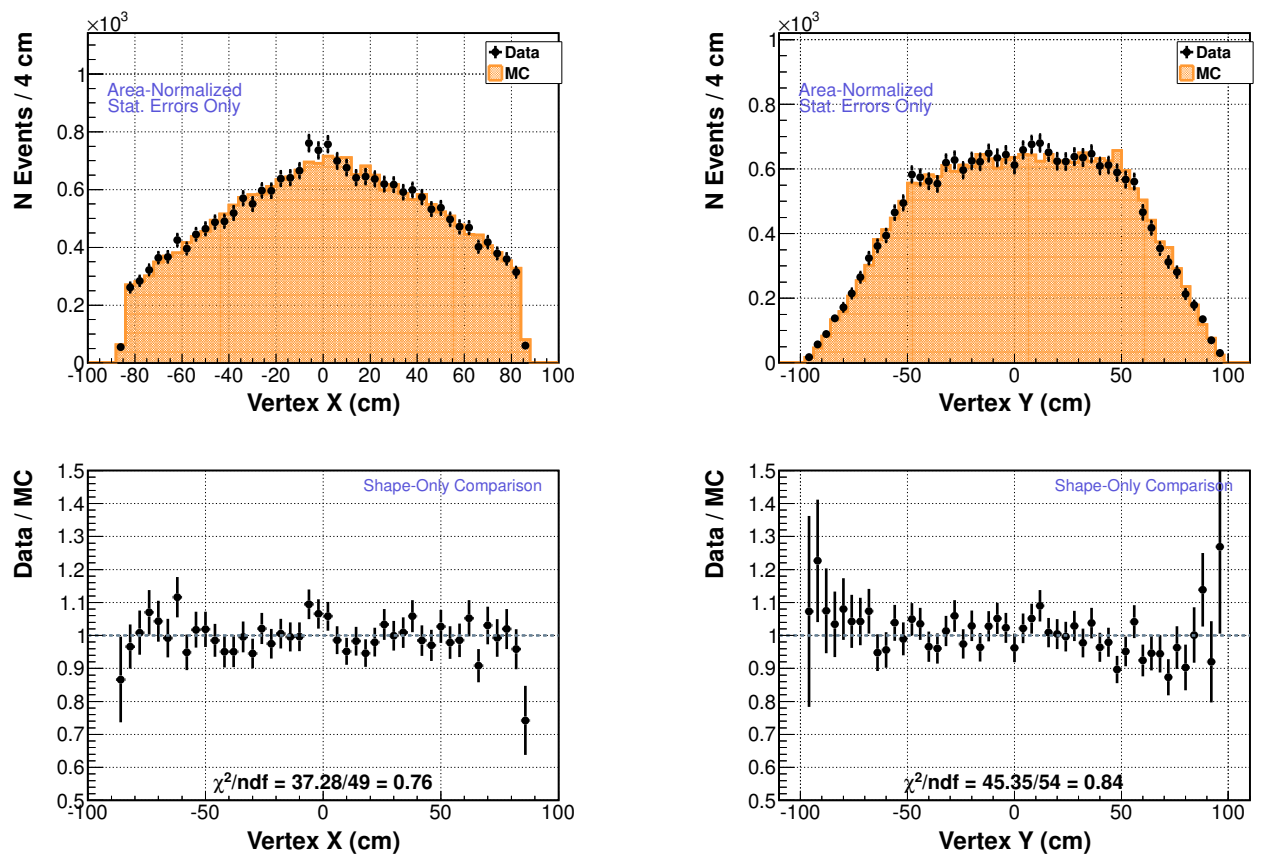

Figure A.3: Vertex distributions of selected events. For explanation, see top of this section. 

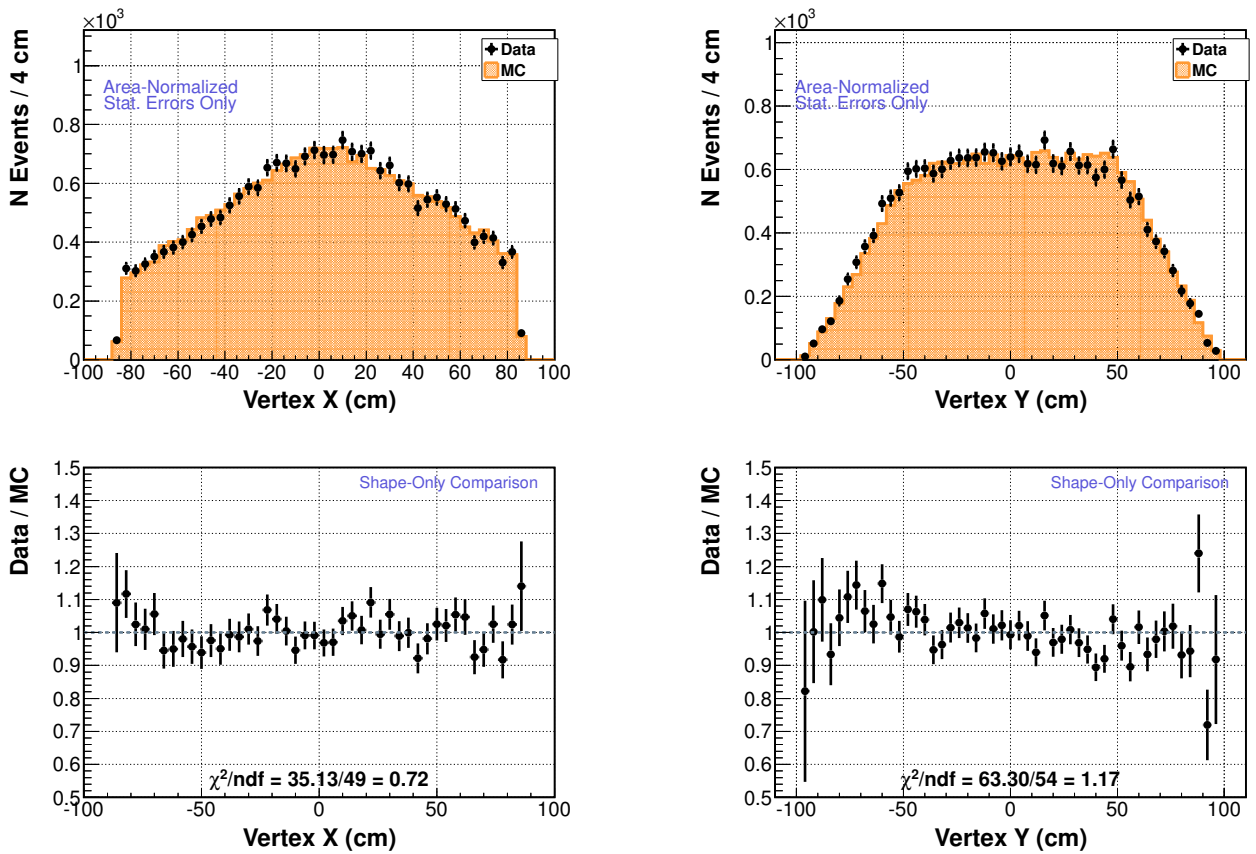

Figure A.3: Vertex distributions of selected events. For explanation, see top of this section. 

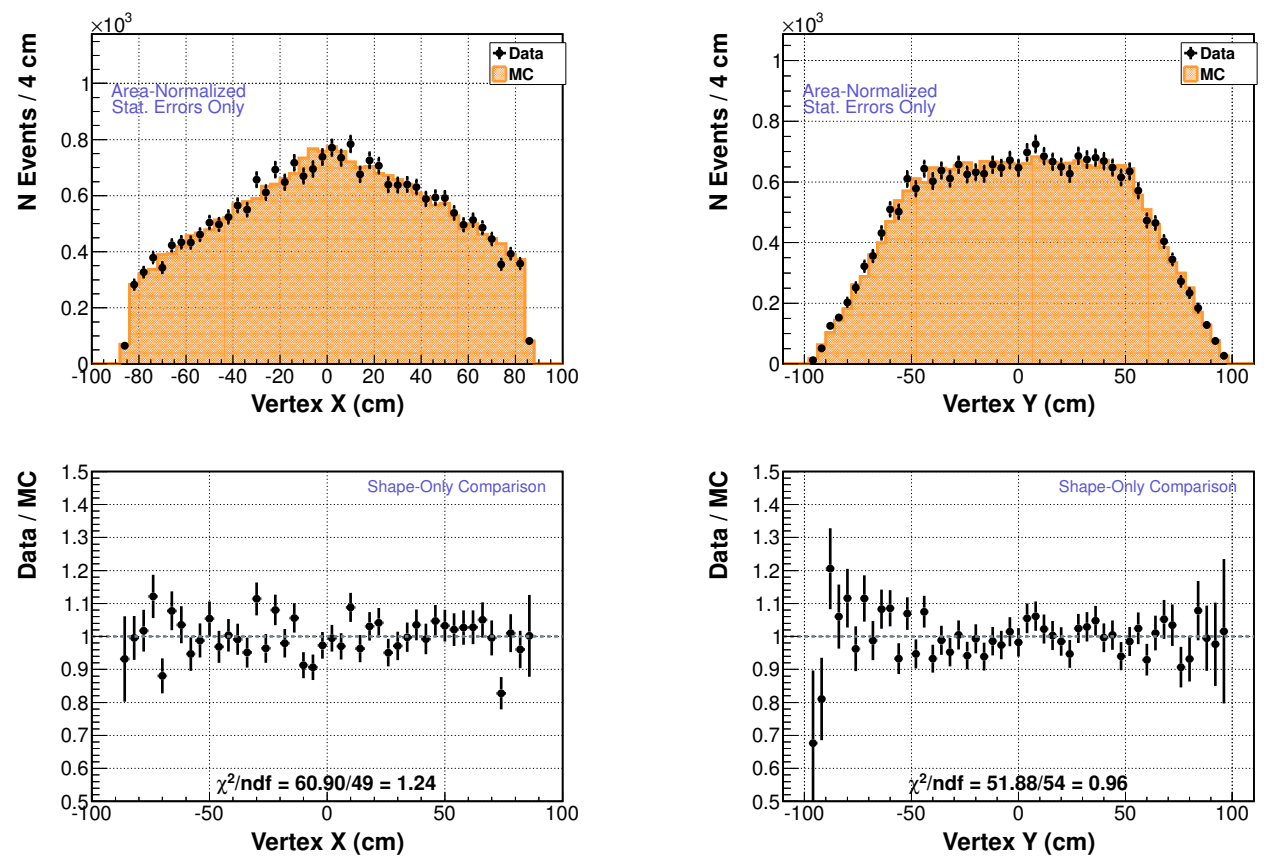

Figure A.3: Vertex distributions of selected events. For explanation, see top of this section. 

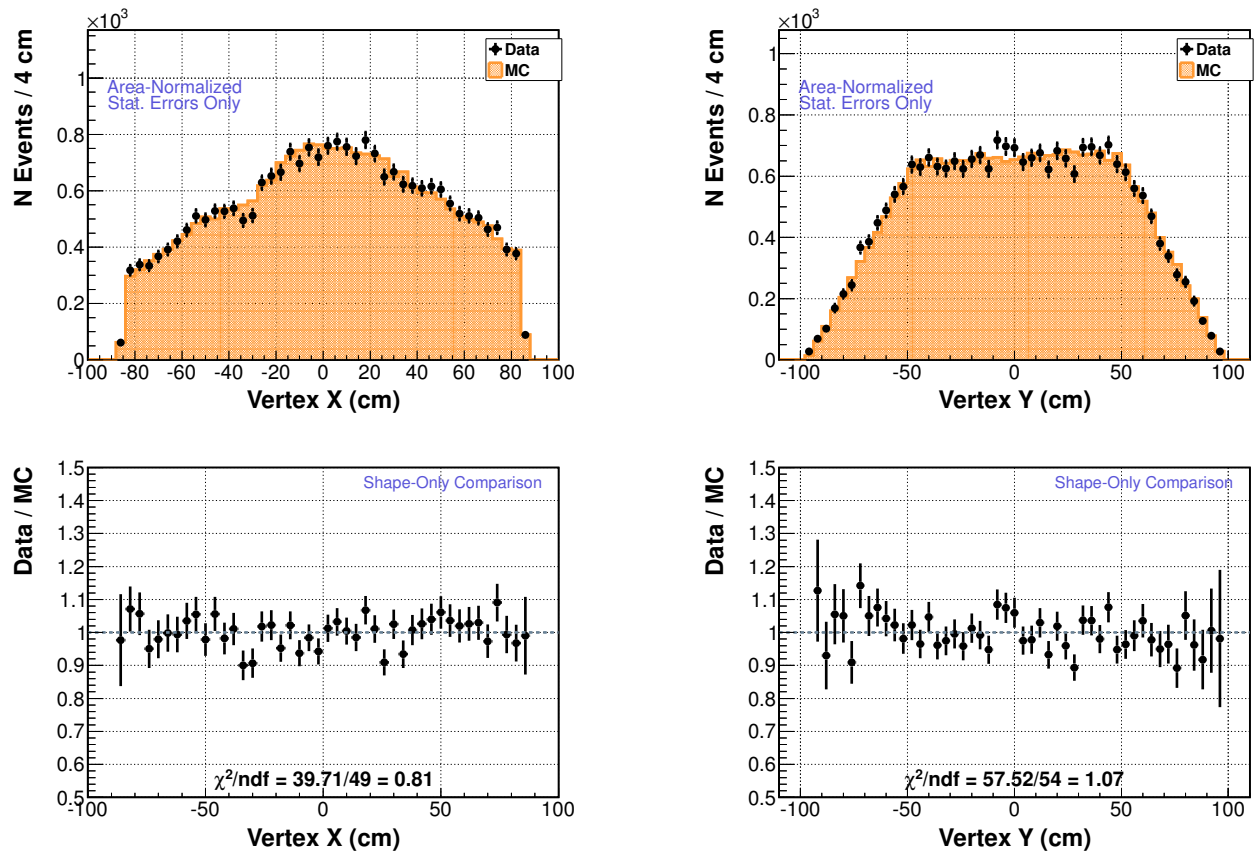

Figure A.3: Vertex distributions of selected events. For explanation, see top of this section. 


\section{A.4 Scintillator Background Prediction}

The event selection criteria for passive targets allows a significant number of events in scintillator to pass. These scintillator events are a background that is subtracted through a datadriven procedure (c.f. Sec. 5.4). Figure A.4 shows the $E_{\text {had }}$-dependent scale factors used to correct for the different reconstruction efficiencies in the tracker and passive target regions. Figures A.5 and A.6 show the accuracy with which the background is subtracted for distributions of $E_{\nu}$ and $x_{b j}$, respectively. Figures A.7 and A.8 show the fractional errors on the predicted distributions of $E_{\nu}$ and $x_{b j}$ for the scintillator background, respectively.

\section{A.4.1 Scale Factors}

\section{A.4.2 Accuracy of Scintillator Background Prediction}

\section{A.4.3 Errors on Scintillator Background Prediction}

\section{A.5 Signal and Background Prediction Distributions}

The figures in this compared the measured and predicted distributions of $E_{\nu}$ (left) and $x_{b j}$ (right). "Sample" means the number of events passing selectrion criteria, "background" is the number of scintillator events contaminating a passive target sample, and "signal" is the background-subtracted sample. Scintillator samples have no such background, so for these the sample and signal are the same. The simulation (MC) histograms were found through full event simulation and reconstruction. The figures in each row are as follows:

- Top - Comparisons of measured sample and background to absolutely-normalized simulation (MC). Statistical error is shown on data points and total systematic error is shown in the shaded simulation band.

- Middle - Data to simulation ratio of signal. Points show statistical error, shaded band 


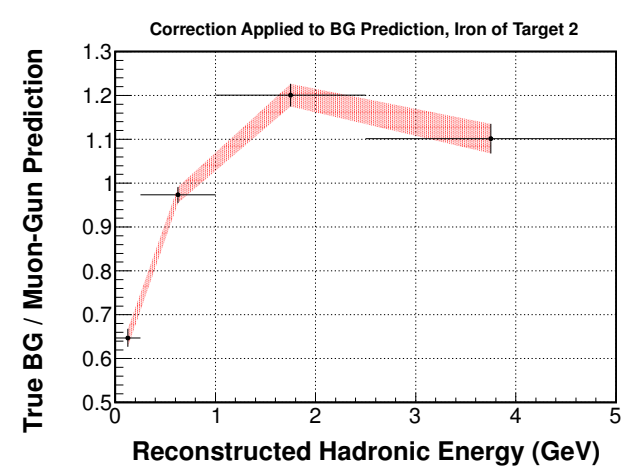

(a) Iron of Target 2

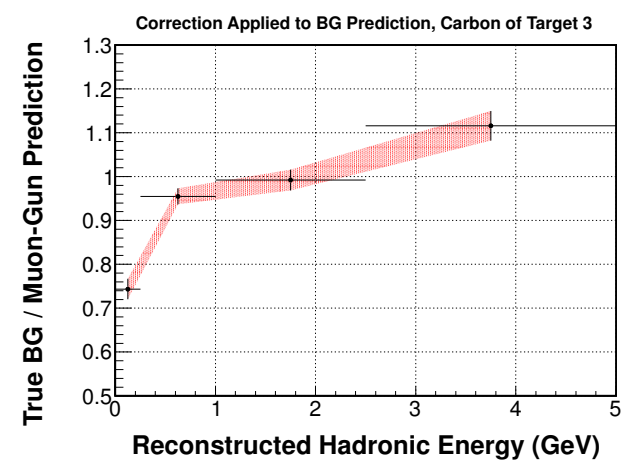

(c) Carbon of Target 3

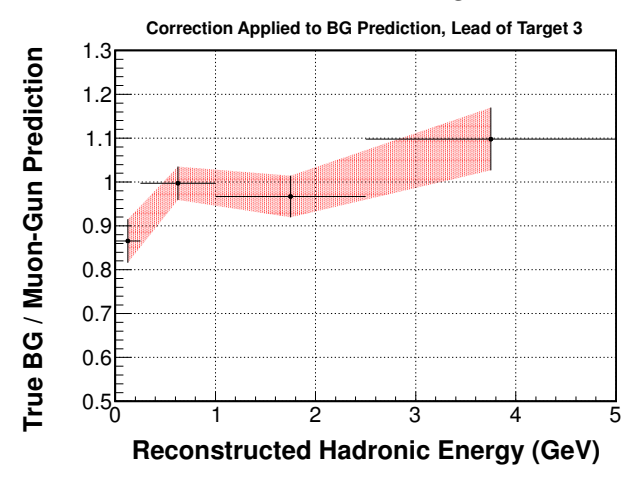

(e) Lead of Target 3

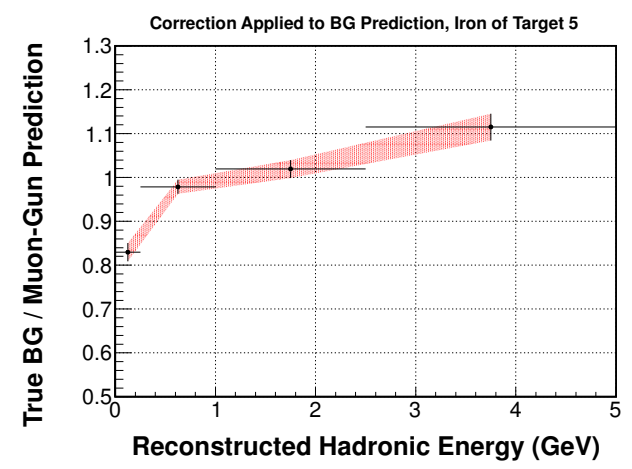

(g) Iron of Target 5

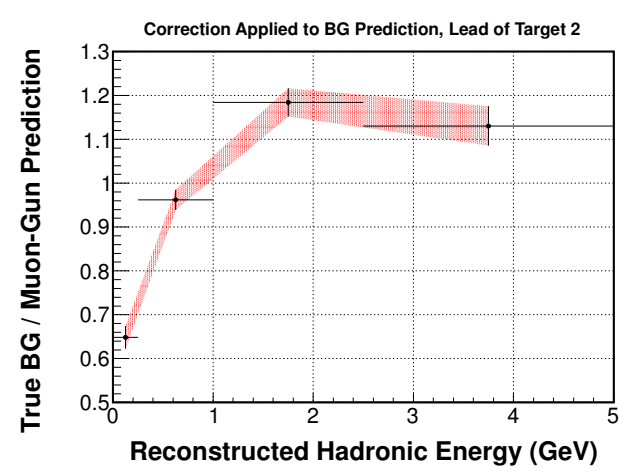

(b) Lead of Target 2

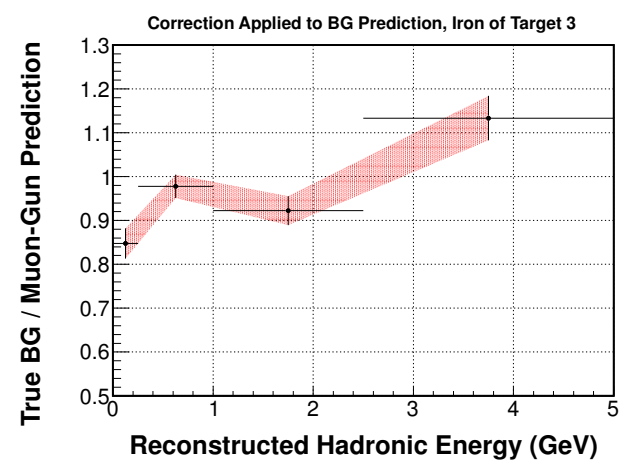

(d) Iron of Target 3

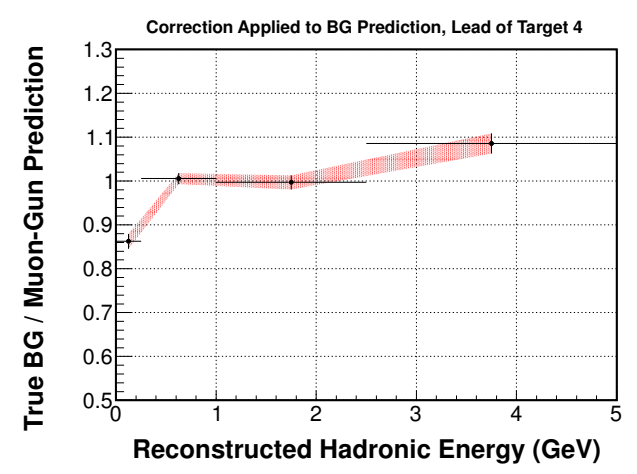

(f) Lead of Target 4

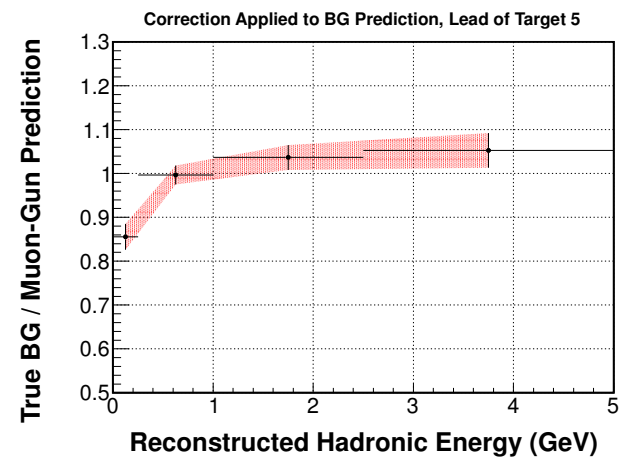

(h) Lead of Target 5

Figure A.4: $E_{h a d}$-dependent scale factor in prediction of background from scintillator in passive targets. The shaded band shows the statistical uncertainty. 


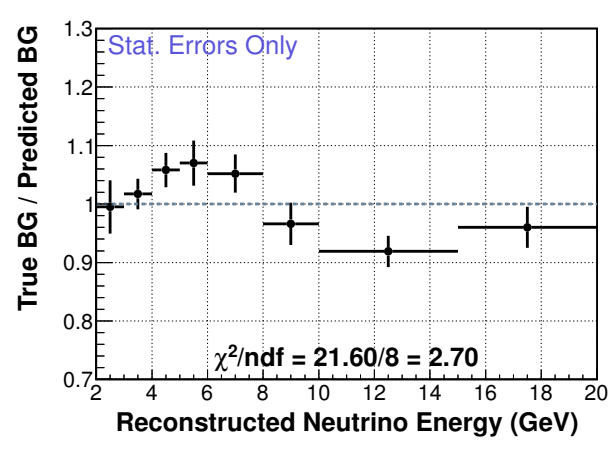

(a) $E_{\nu}$, Iron of Target 2

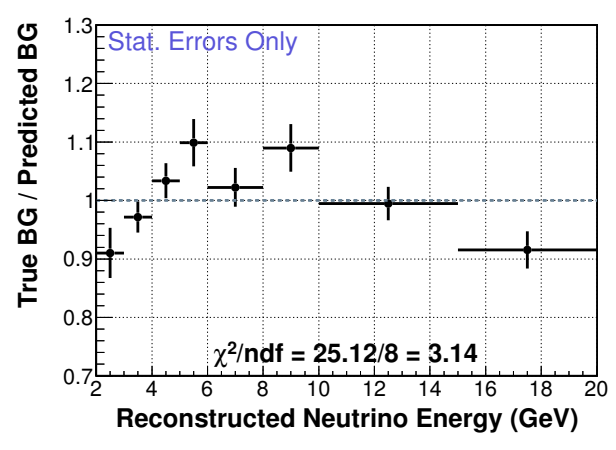

(c) $E_{\nu}$, Carbon of Target 3

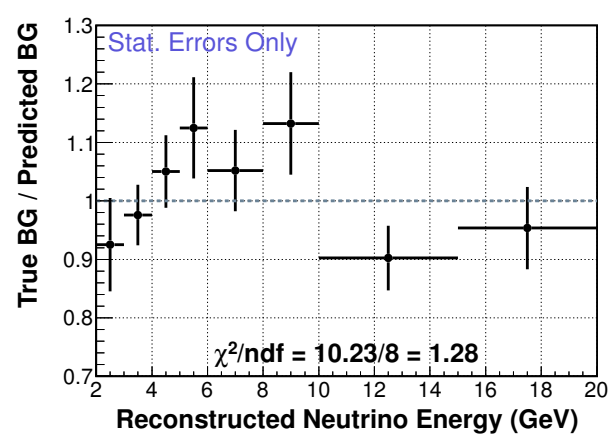

(e) $E_{\nu}$, Lead of Target 3

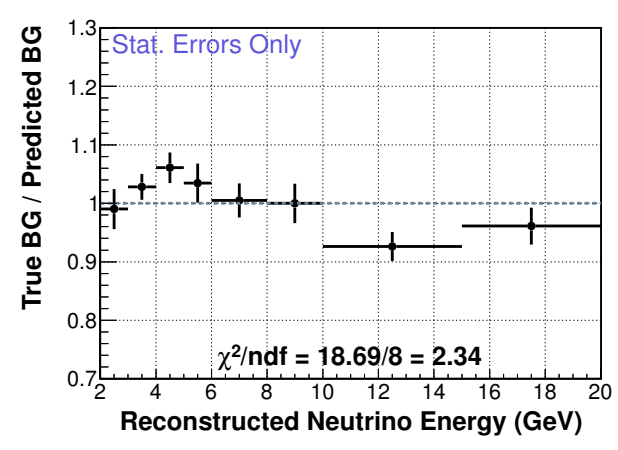

(g) $E_{\nu}$, Iron of Target 5

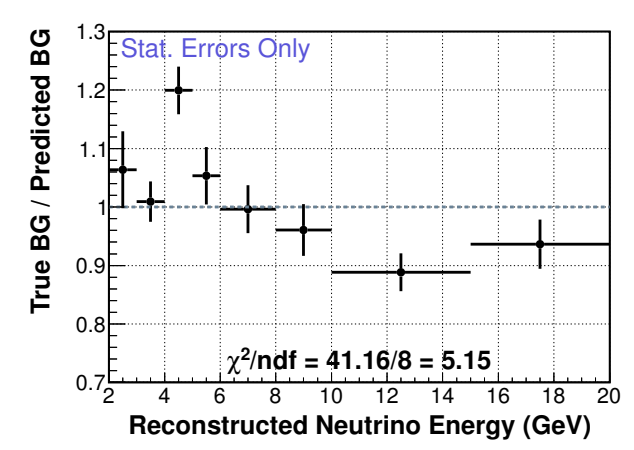

(b) $E_{\nu}$, Lead of Target 2

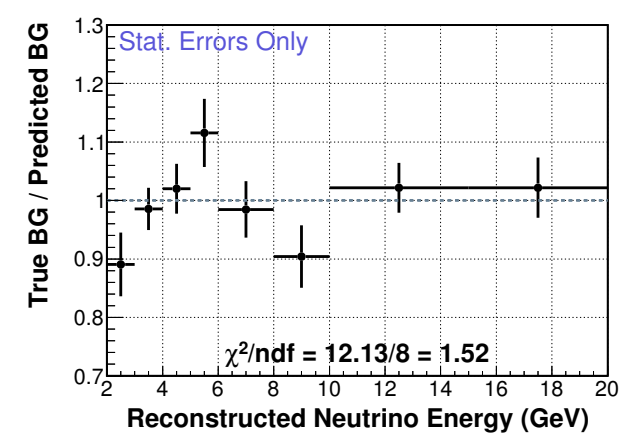

(d) $E_{\nu}$, Iron of Target 3

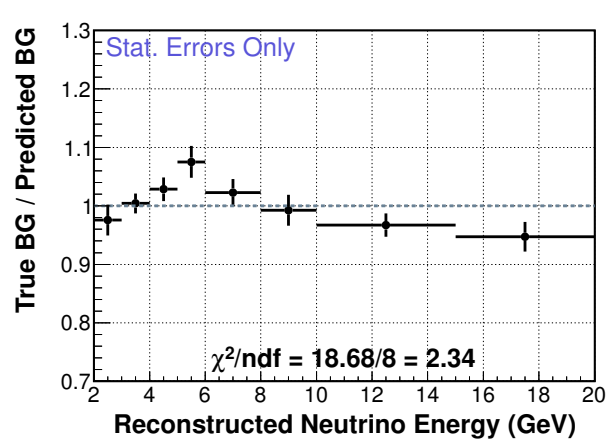

(f) $E_{\nu}$, Lead of Target 4

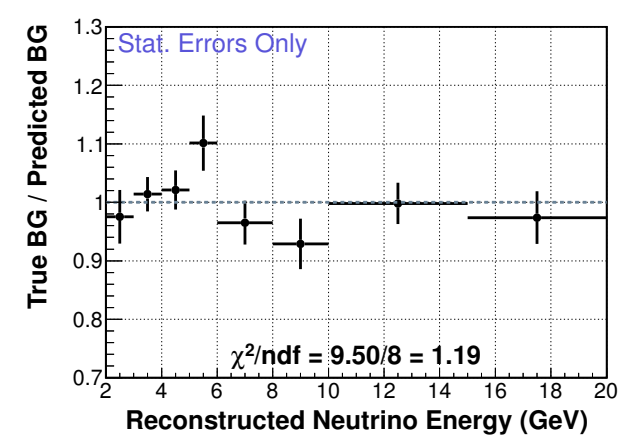

(h) $E_{\nu}$, Lead of Target 5

Figure A.5: Accuracy of the prediction of scintillator contamination for $E_{\nu}$ distributions. The $y$ axis is the ratio of the true background to the predicted background. The error bars are the statistical error of the Monte Carlo. 


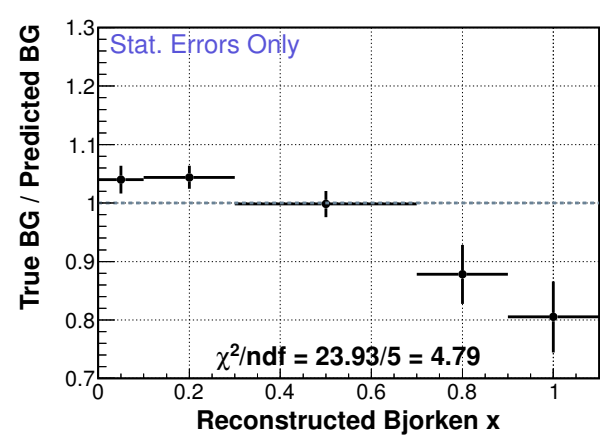

(a) $x_{b j}$, Iron of Target 2

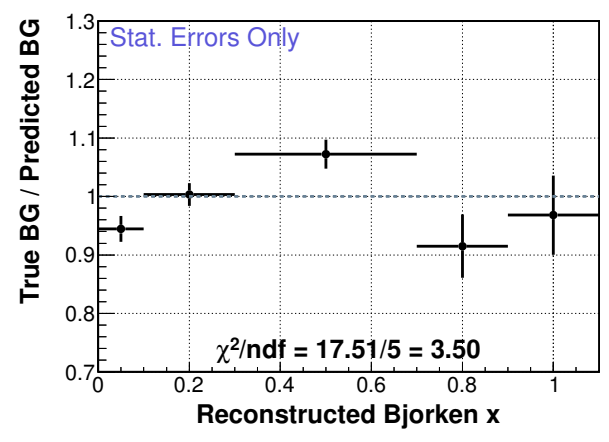

(c) $x_{b j}$, Carbon of Target 3

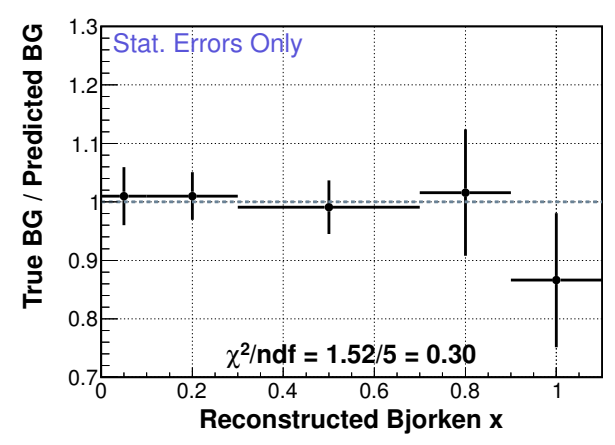

(e) $x_{b j}$, Lead of Target 3

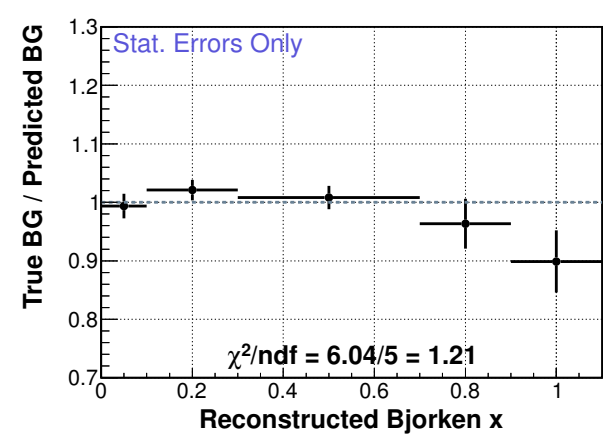

(g) $x_{b j}$, Iron of Target 5

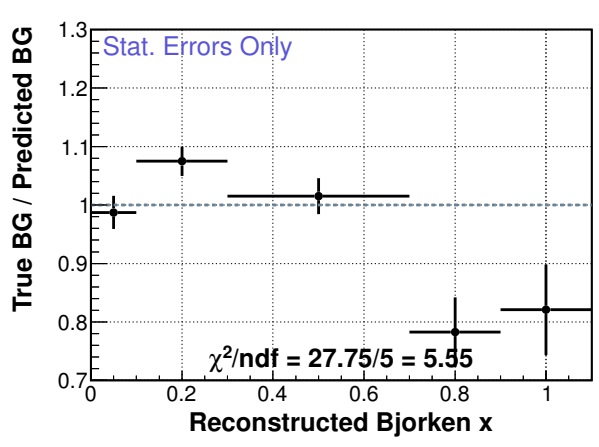

(b) $x_{b j}$, Lead of Target 2

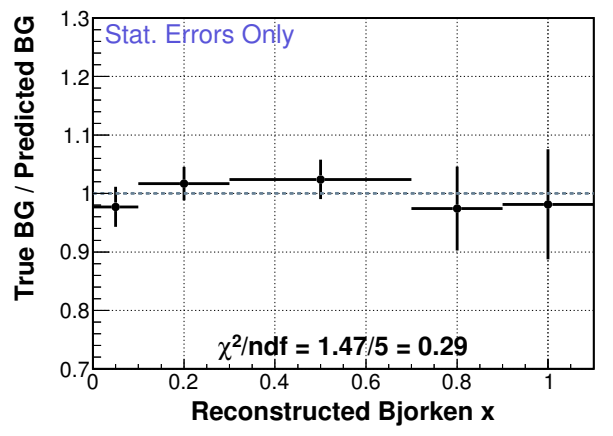

(d) $x_{b j}$, Iron of Target 3

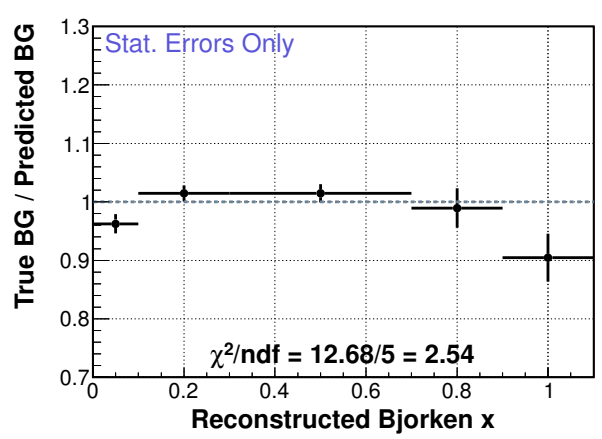

(f) $x_{b j}$, Lead of Target 4

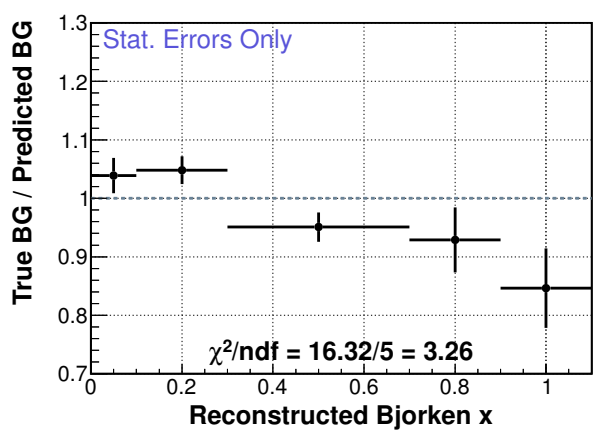

(h) $x_{b j}$, Lead of Target 5

Figure A.6: Accuracy of the prediction of scintillator contamination for $x_{b j}$ distributions. The $\mathrm{y}$ axis is the ratio of the true background to the predicted background. The error bars are the statistical error of the Monte Carlo. 


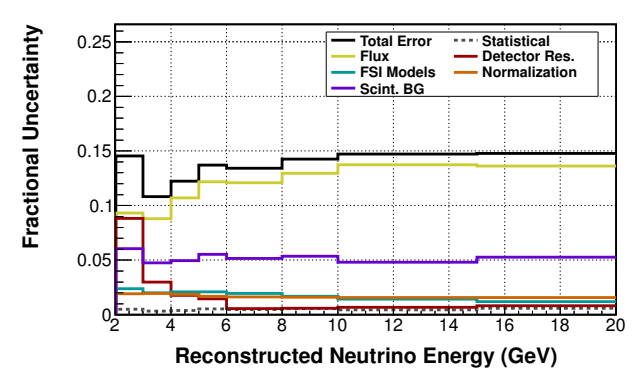

(a) $E_{\nu}$, Iron of Target 2

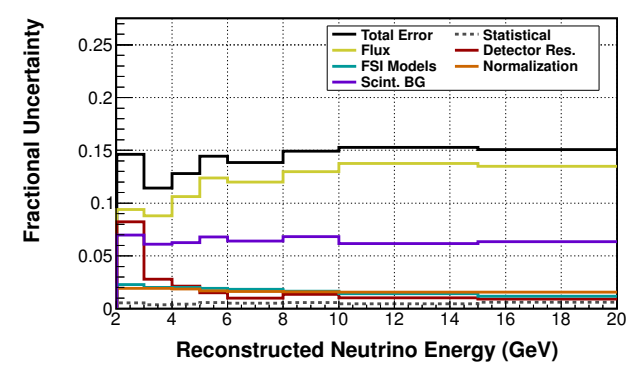

(c) $E_{\nu}$, Carbon of Target 3

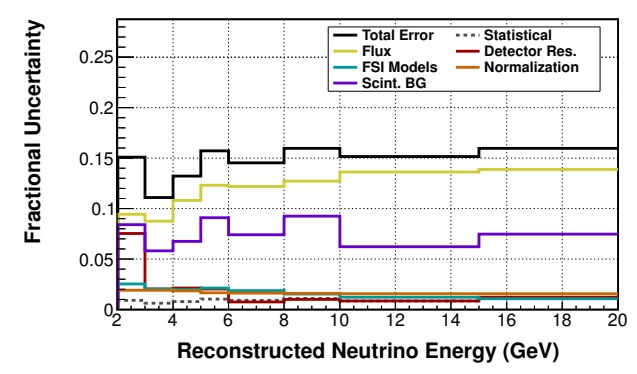

(e) $E_{\nu}$, Lead of Target 3

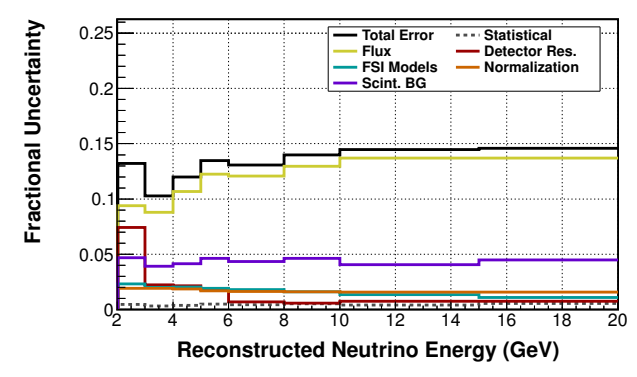

(g) $E_{\nu}$, Iron of Target 5

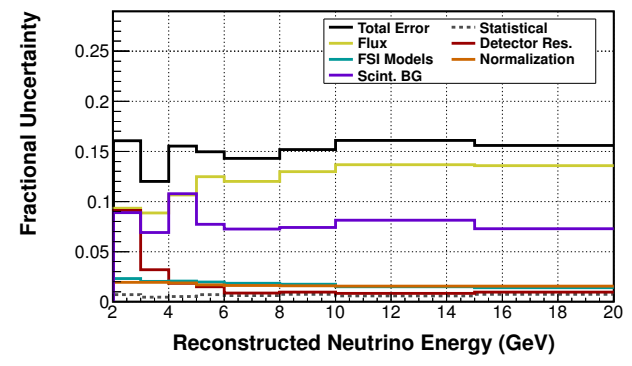

(b) $E_{\nu}$, Lead of Target 2

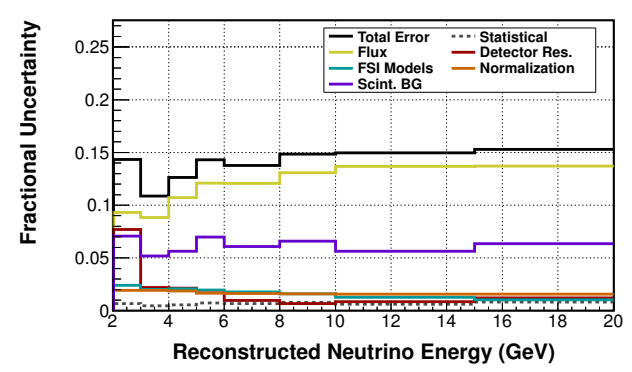

(d) $E_{\nu}$, Iron of Target 3

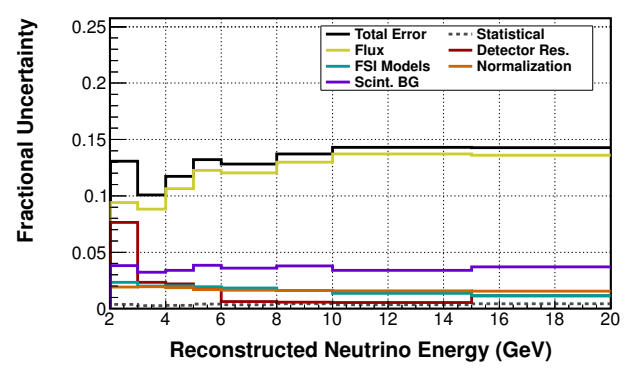

(f) $E_{\nu}$, Lead of Target 4

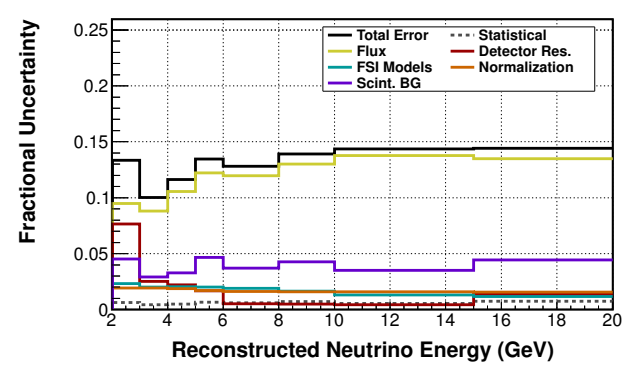

(h) $E_{\nu}$, Lead of Target 5

Figure A.7: Fractional errors on the prediction of the distribution of scintillator background in passive targets. 


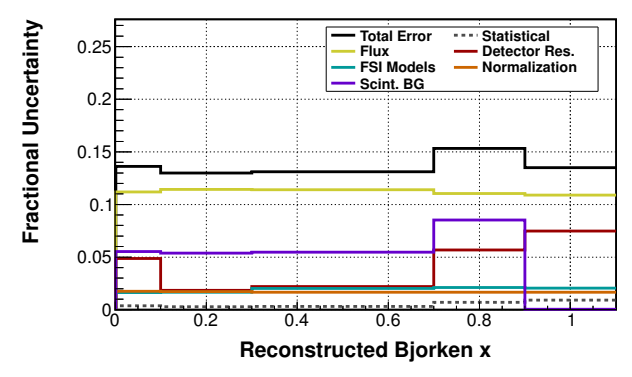

(a) $x_{b j}$, Iron of Target 2

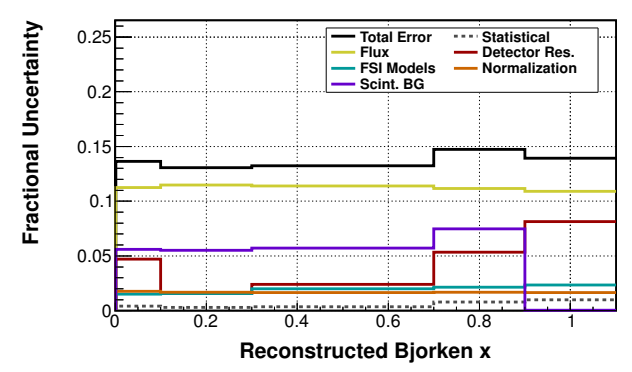

(c) $x_{b j}$, Carbon of Target 3

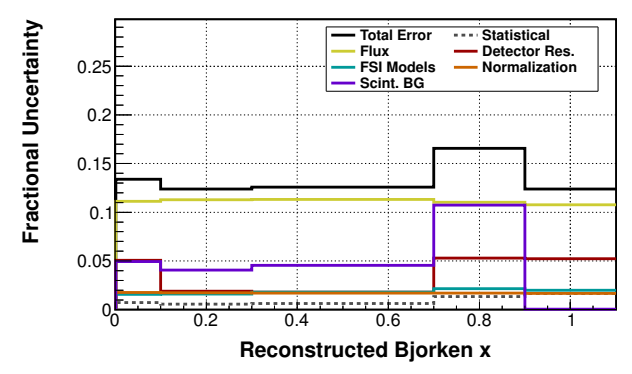

(e) $x_{b j}$, Lead of Target 3

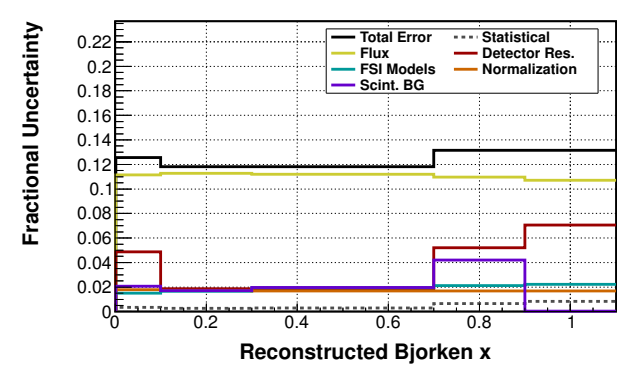

(g) $x_{b j}$, Iron of Target 5

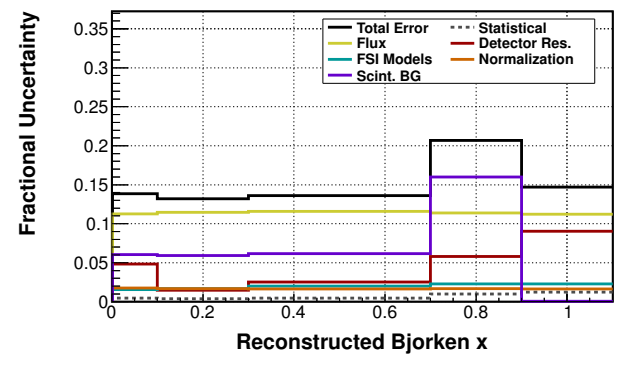

(b) $x_{b j}$, Lead of Target 2

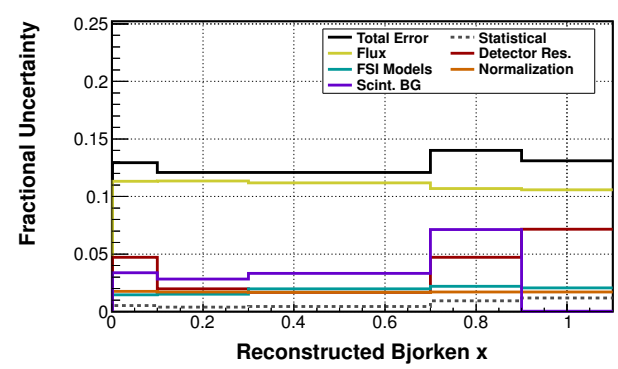

(d) $x_{b j}$, Iron of Target 3

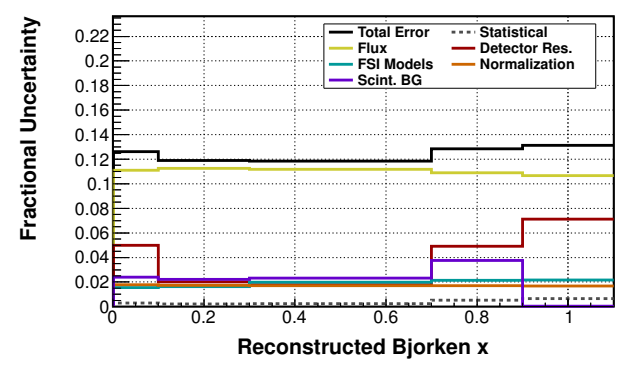

(f) $x_{b j}$, Lead of Target 4

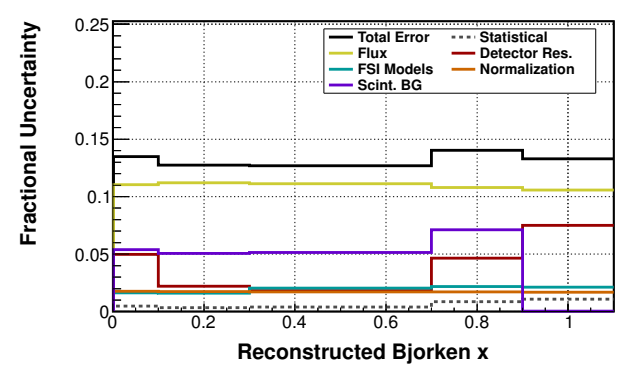

(h) $x_{b j}$, Lead of Target 5

Figure A.8: Fractional errors on the prediction of the distribution of scintillator background in passive targets. 
centered on 1 represents full systematic error, and the $\chi^{2}$ calculation accounts for bin-tobin correlations.

- Bottom - Fractional errors on the signal. The total error is all sources summed in quadrature.

Each page shows a different event sample.
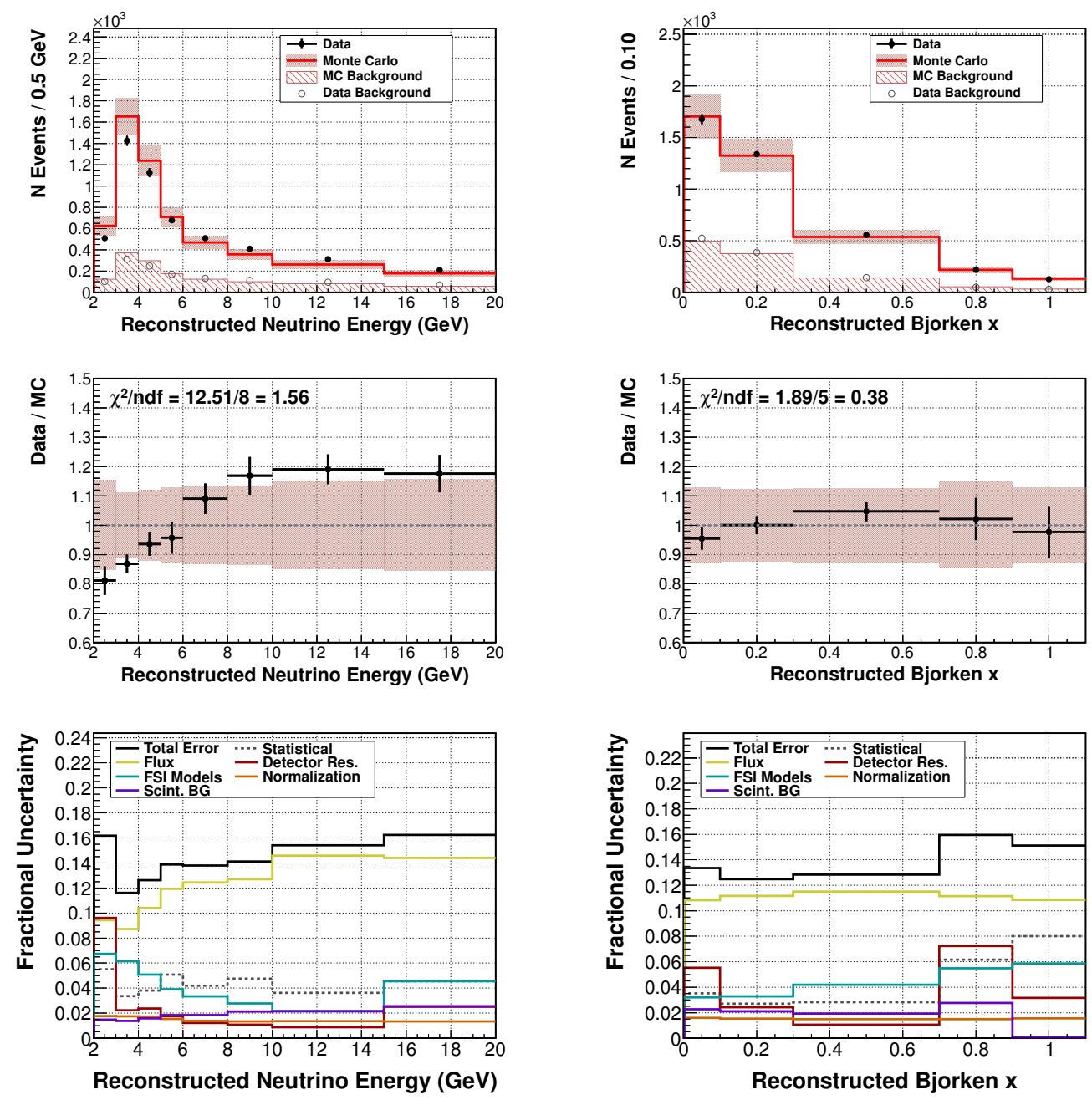

(a) Target 2 iron

Figure A.9: Signal and background distribution plots. For explanation, see top of this section. 

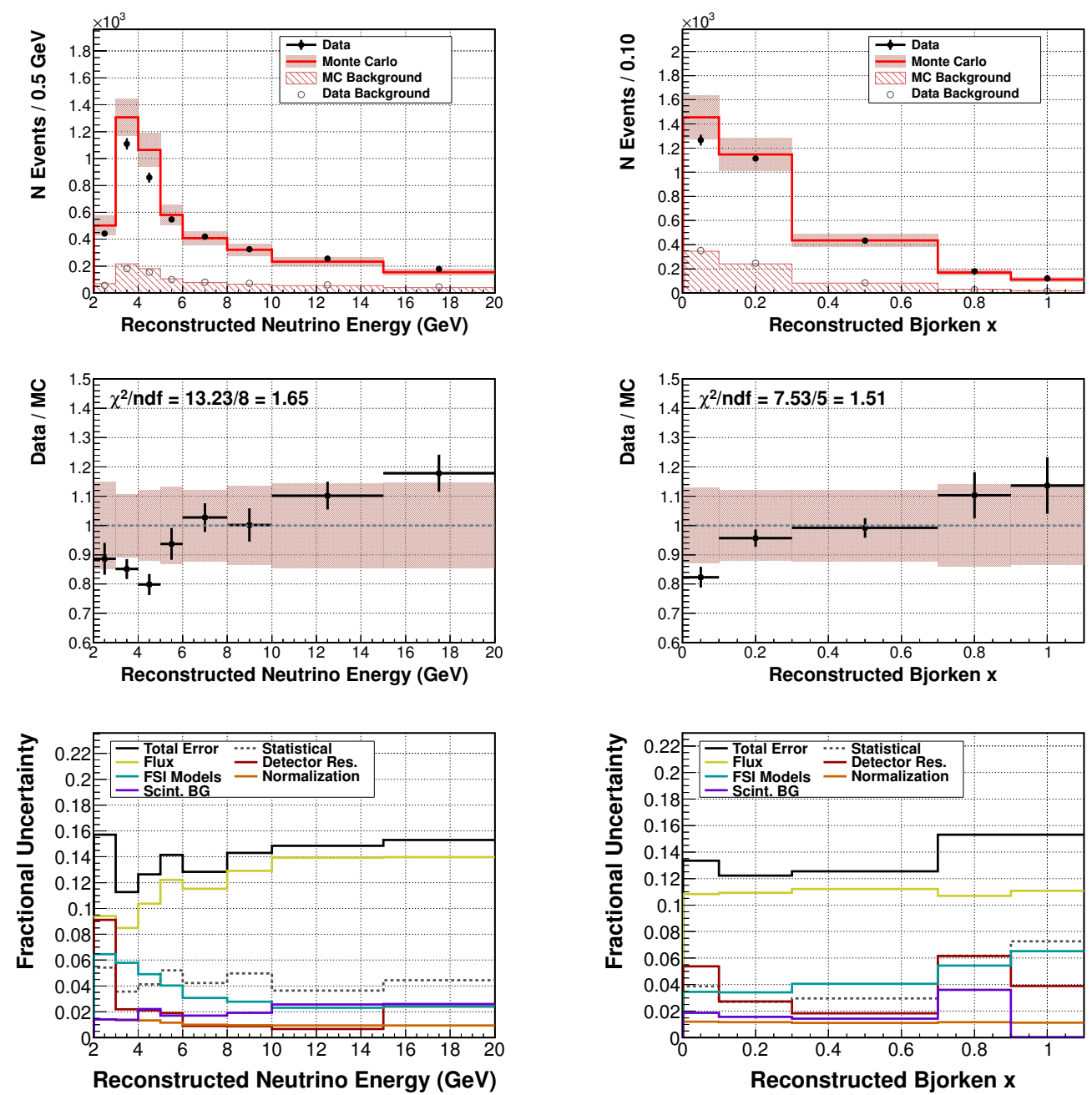

(b) Target 2 lead

Figure A.9: Signal and background distribution plots. For explanation, see top of this section. 

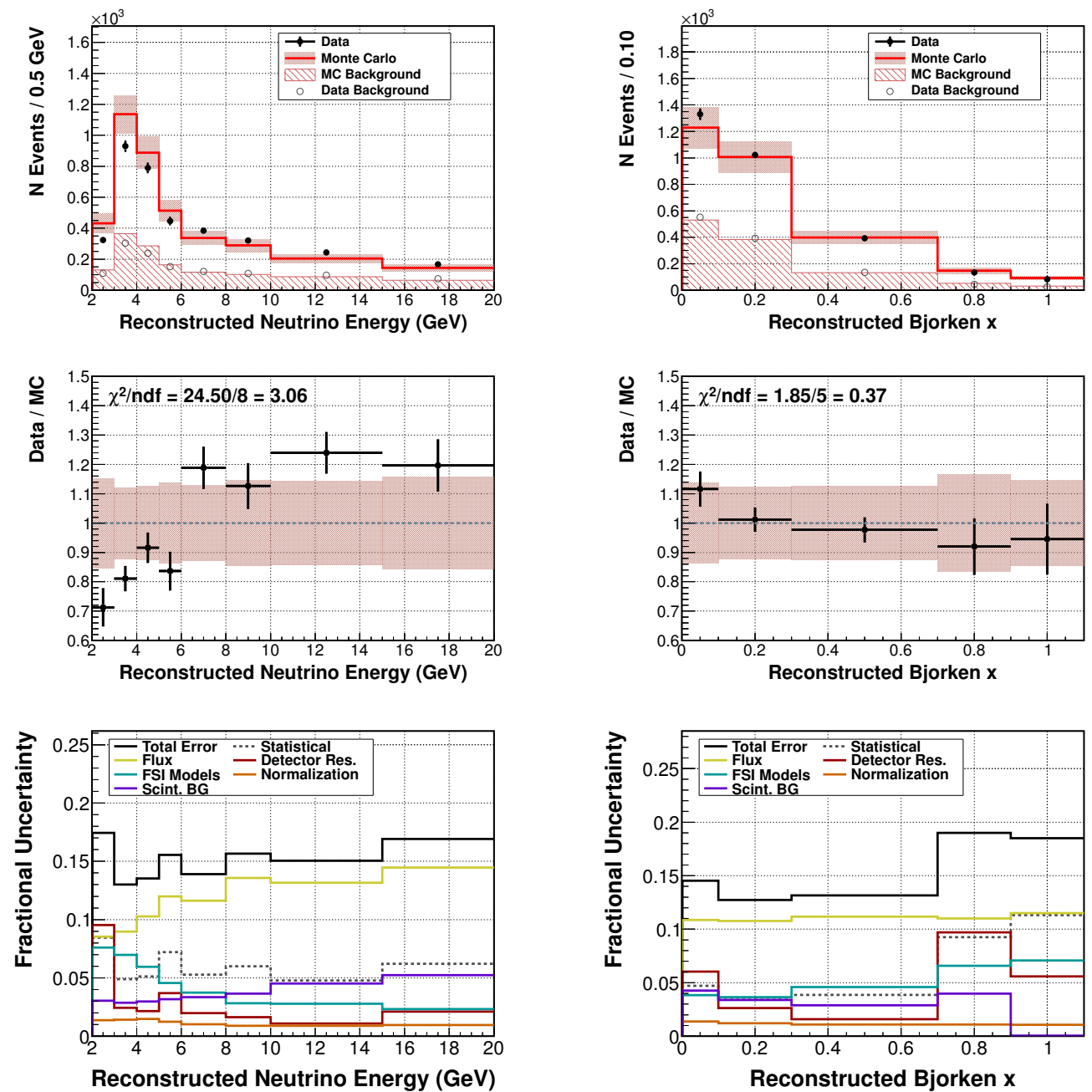

(c) Target 3 carbon

Figure A.9: Signal and background distribution plots. For explanation, see top of this section. 

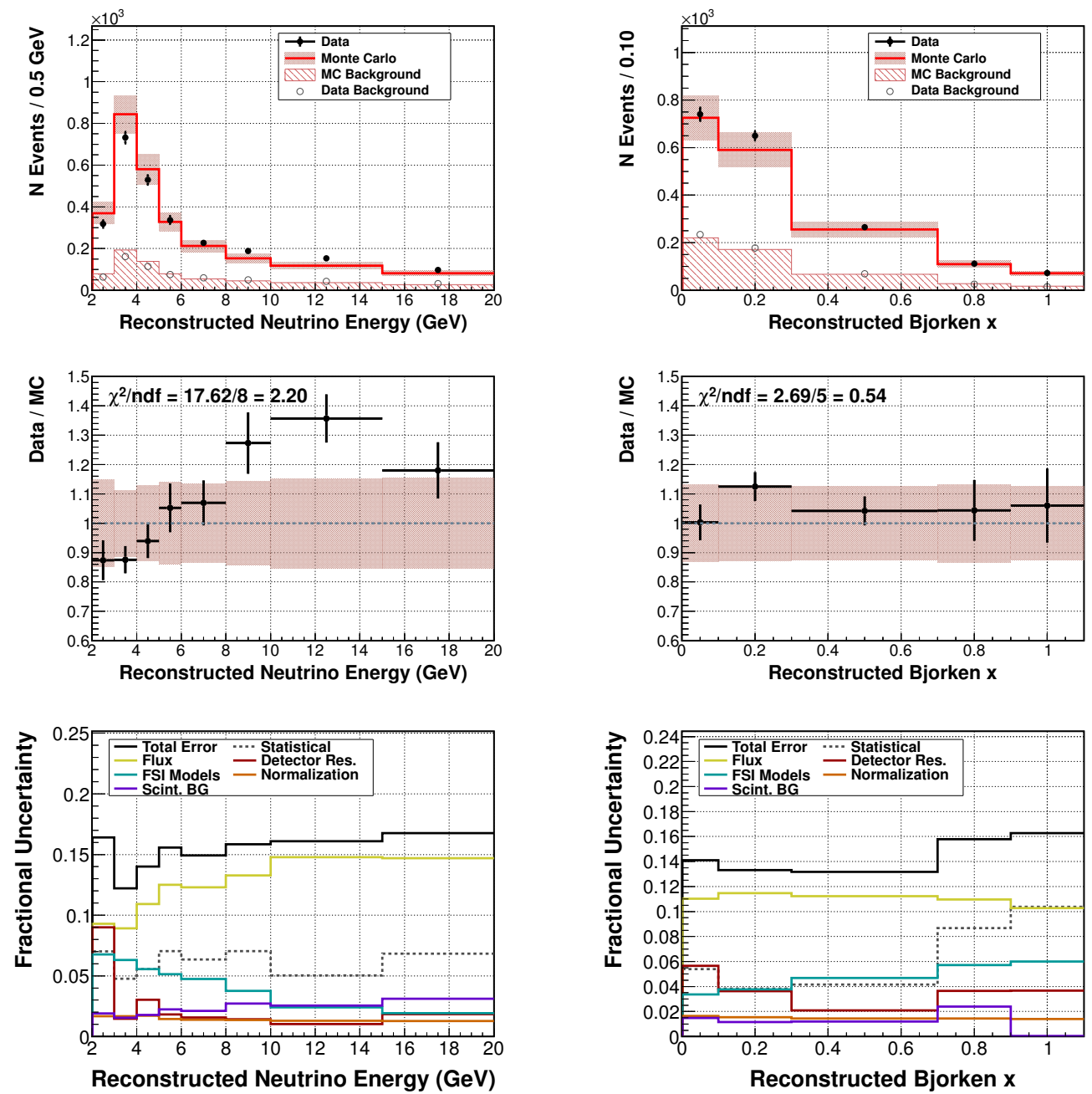

(d) Target 3 iron

Figure A.9: Signal and background distribution plots. For explanation, see top of this section. 

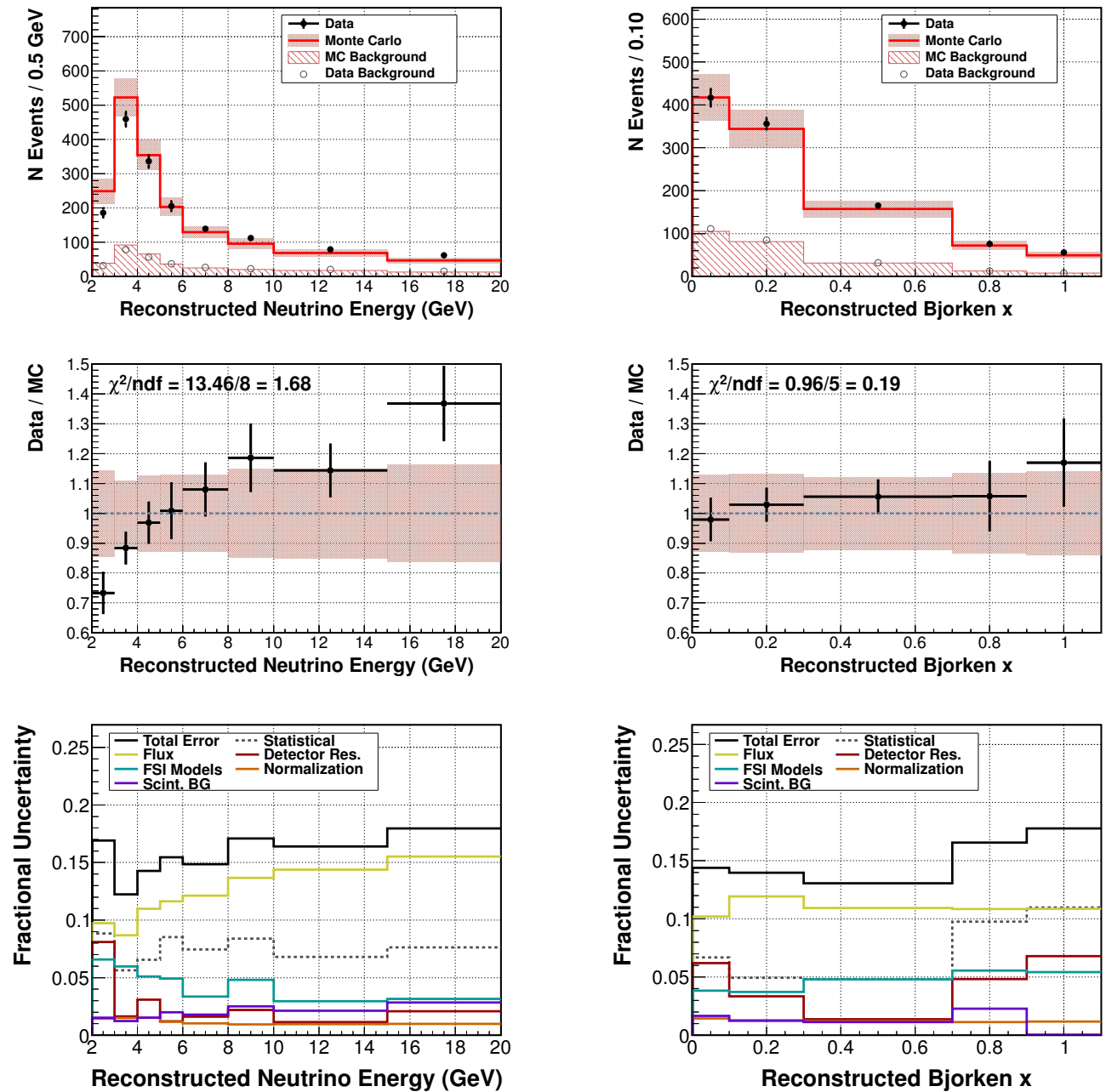

(e) Target 3 lead

Figure A.9: Signal and background distribution plots. For explanation, see top of this section. 

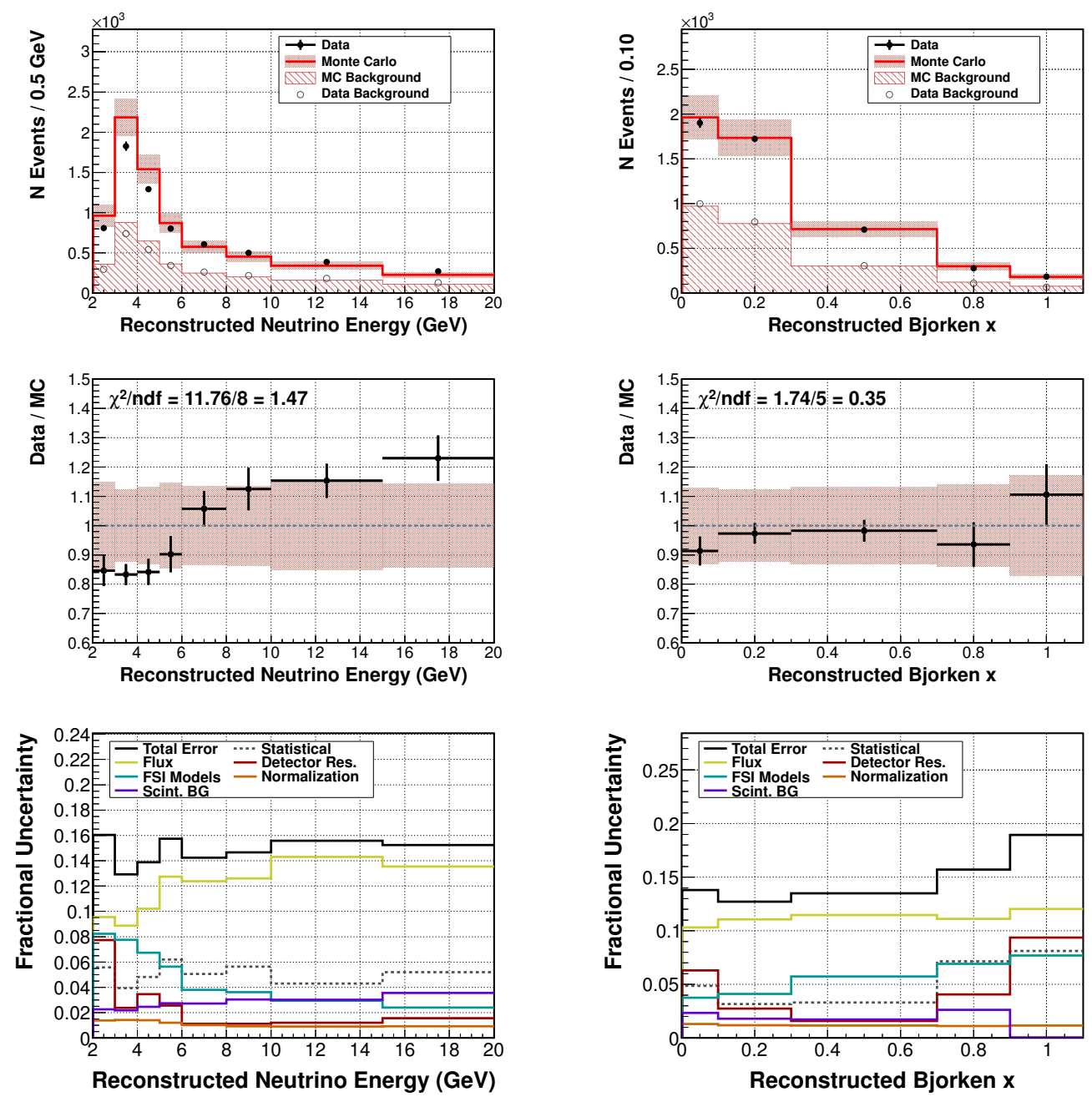

(f) Target 4 lead

Figure A.9: Signal and background distribution plots. For explanation, see top of this section. 

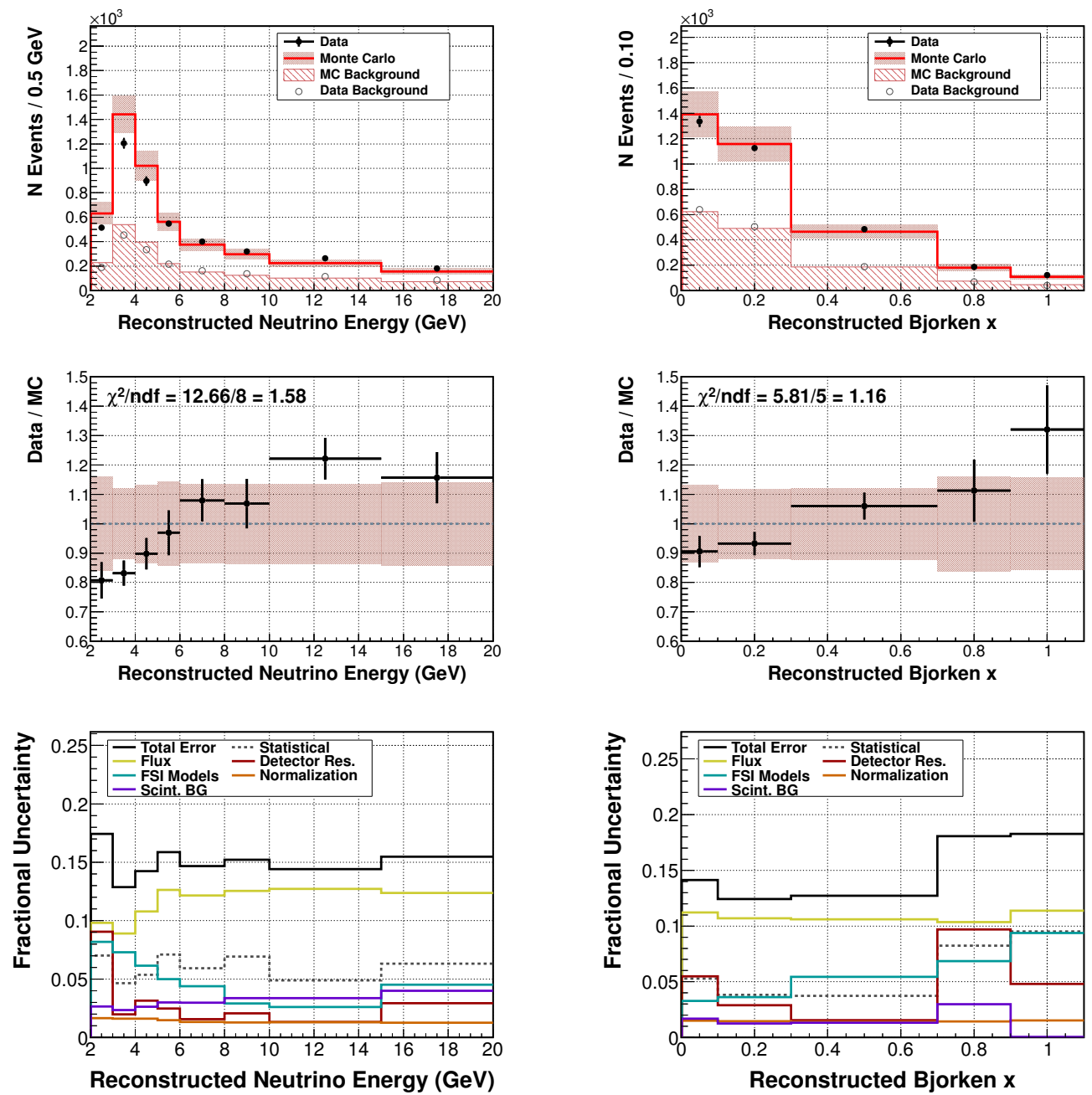

(g) Target 5 iron

Figure A.9: Signal and background distribution plots. For explanation, see top of this section. 

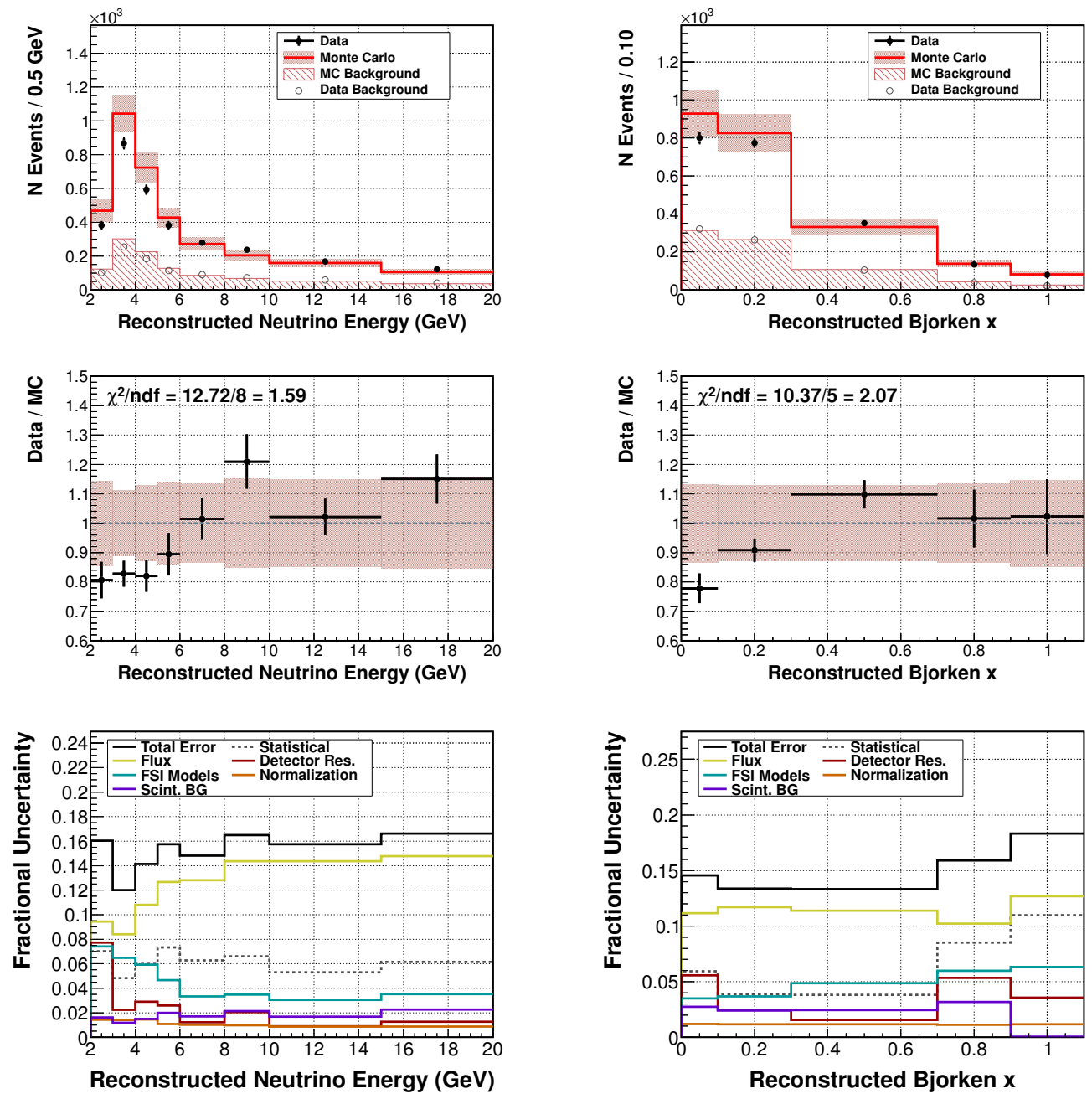

(h) Target 5 lead

Figure A.9: Signal and background distribution plots. For explanation, see top of this section. 

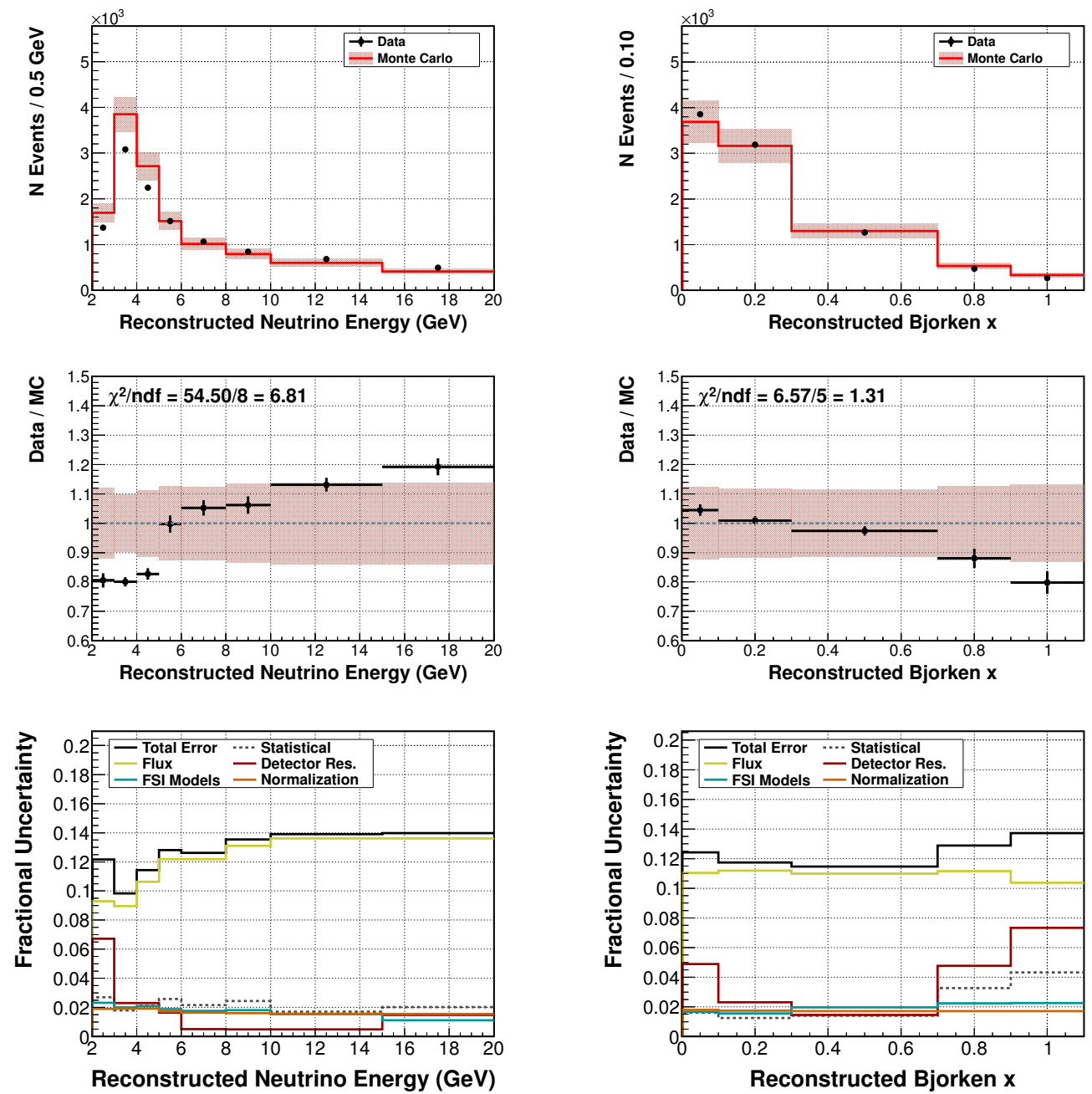

(i) Tracker modules 27-32

Figure A.9: Signal and background distribution plots. For explanation, see top of this section. 

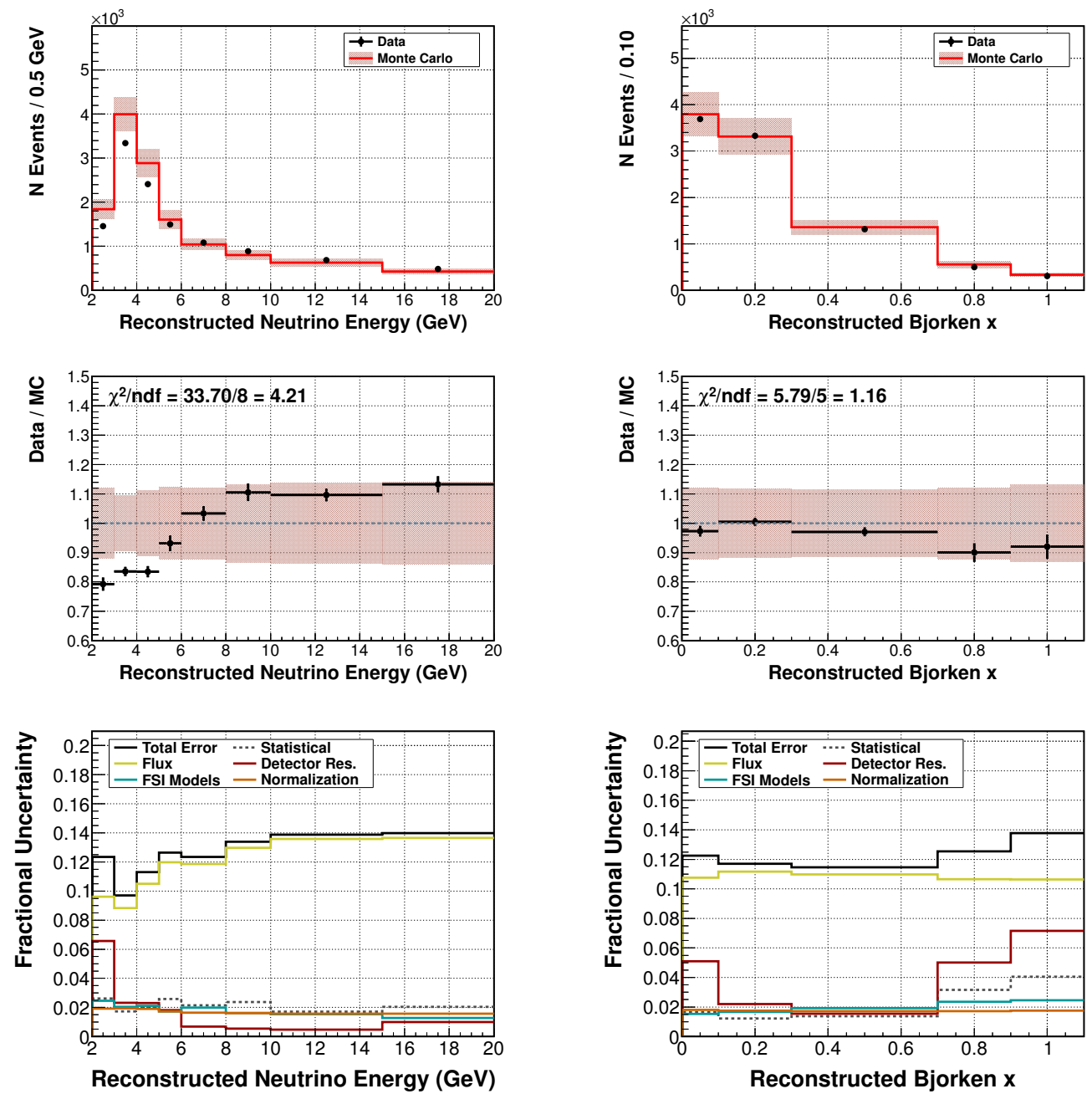

(j) Tracker modules 33-38

Figure A.9: Signal and background distribution plots. For explanation, see top of this section. 

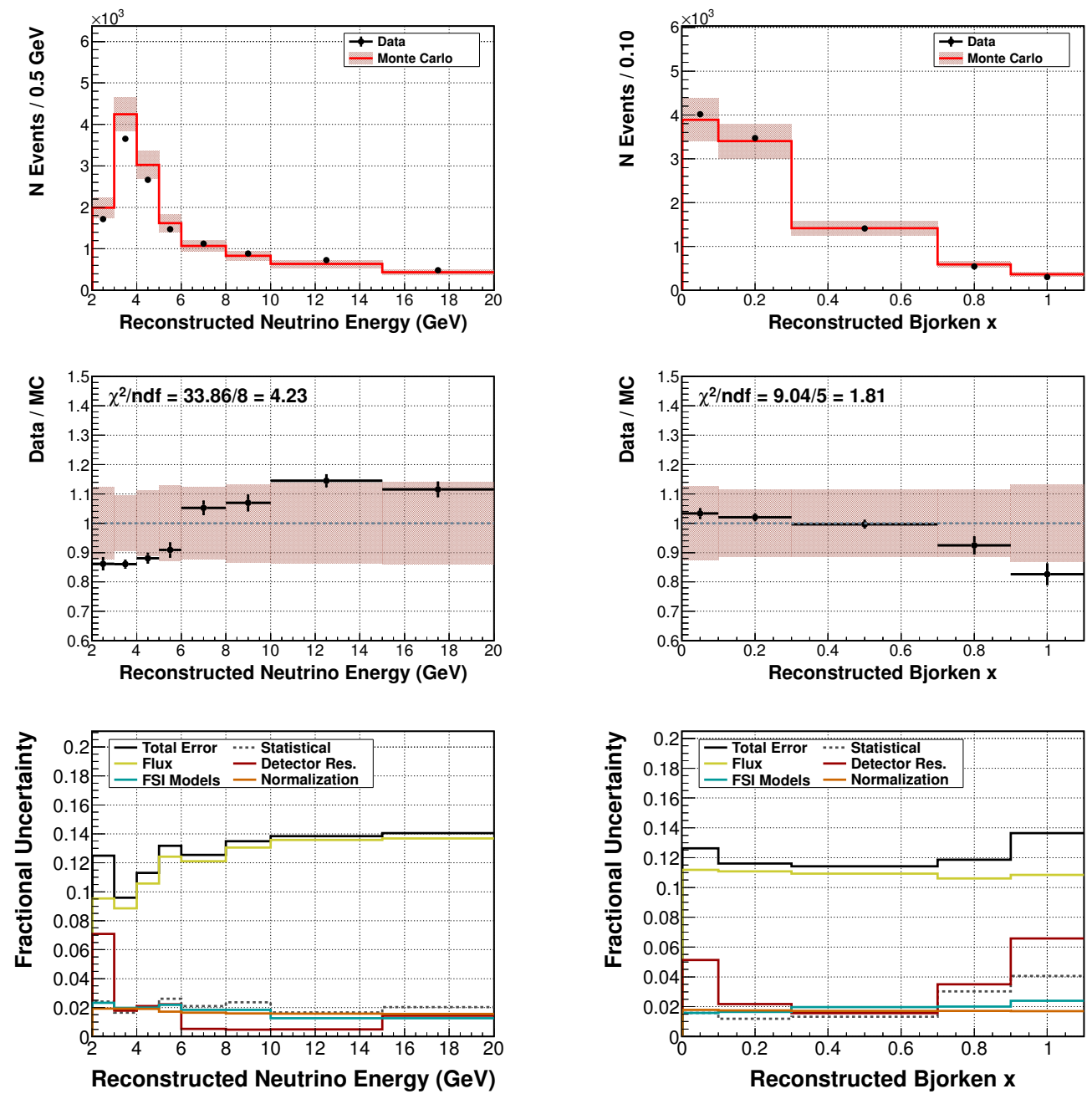

(k) Tracker modules 39-44

Figure A.9: Signal and background distribution plots. For explanation, see top of this section. 

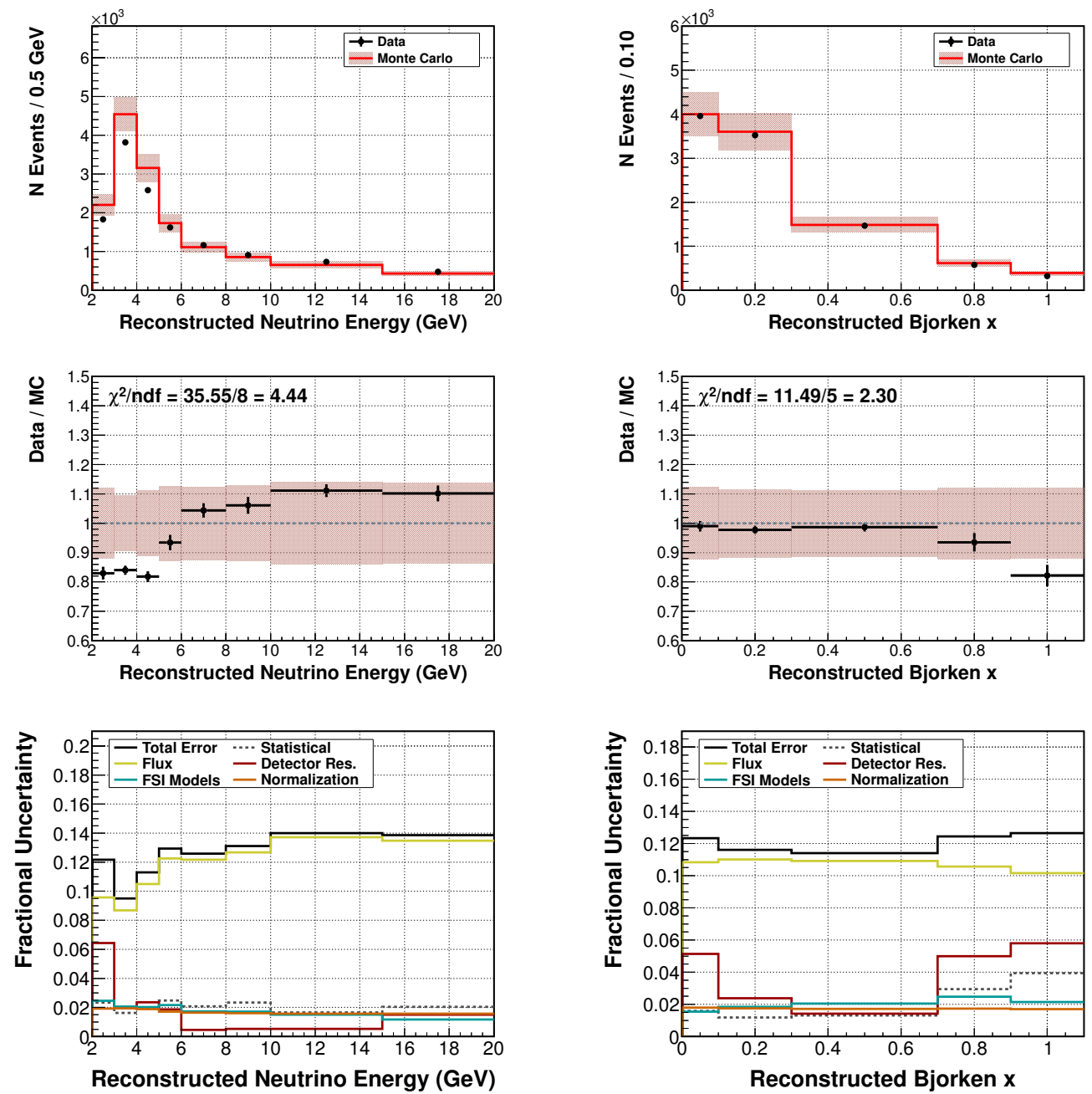

(1) Tracker modules $45-50$

Figure A.9: Signal and background distribution plots. For explanation, see top of this section. 

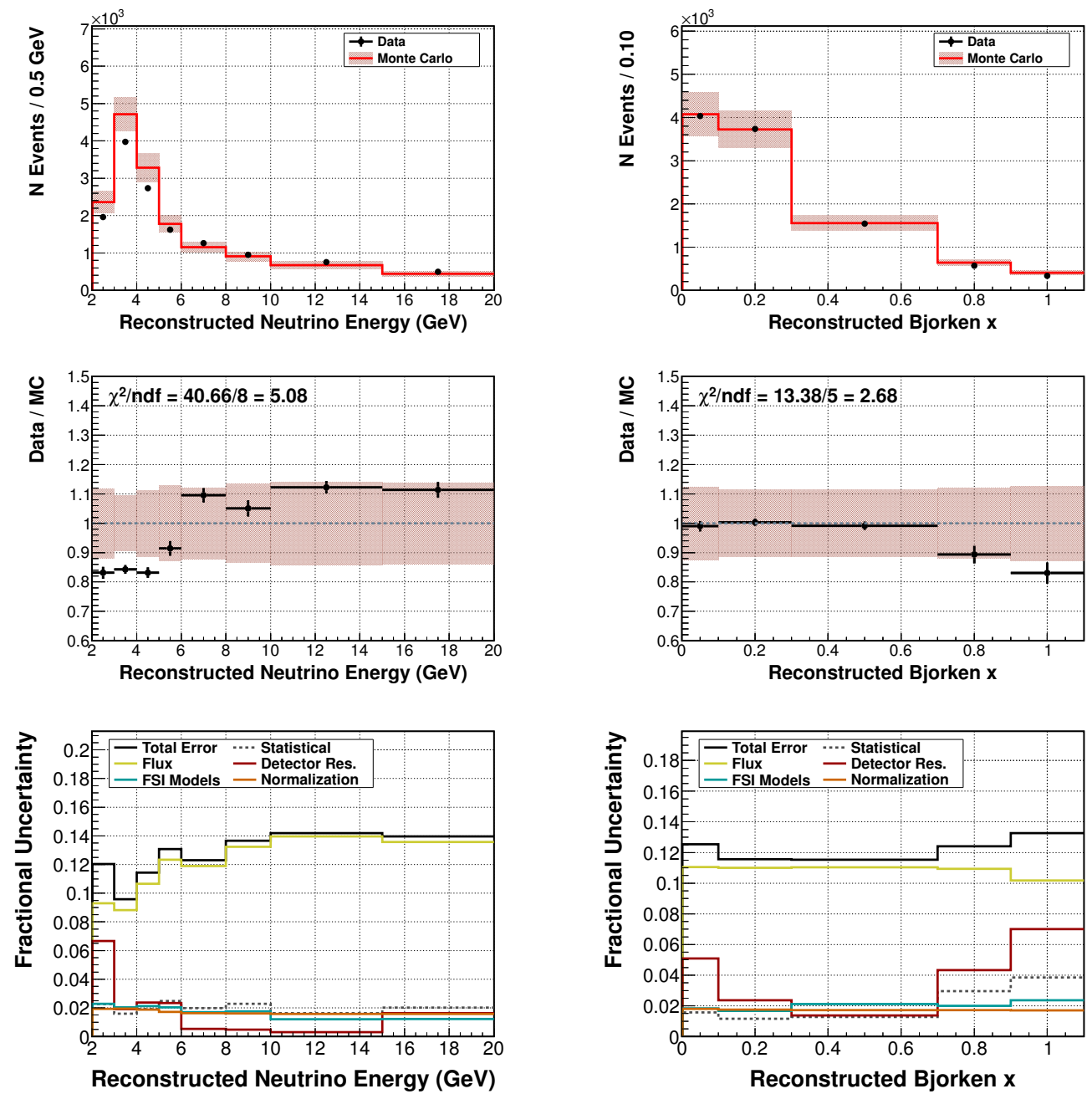

(m) Tracker modules 51-56

Figure A.9: Signal and background distribution plots. For explanation, see top of this section. 

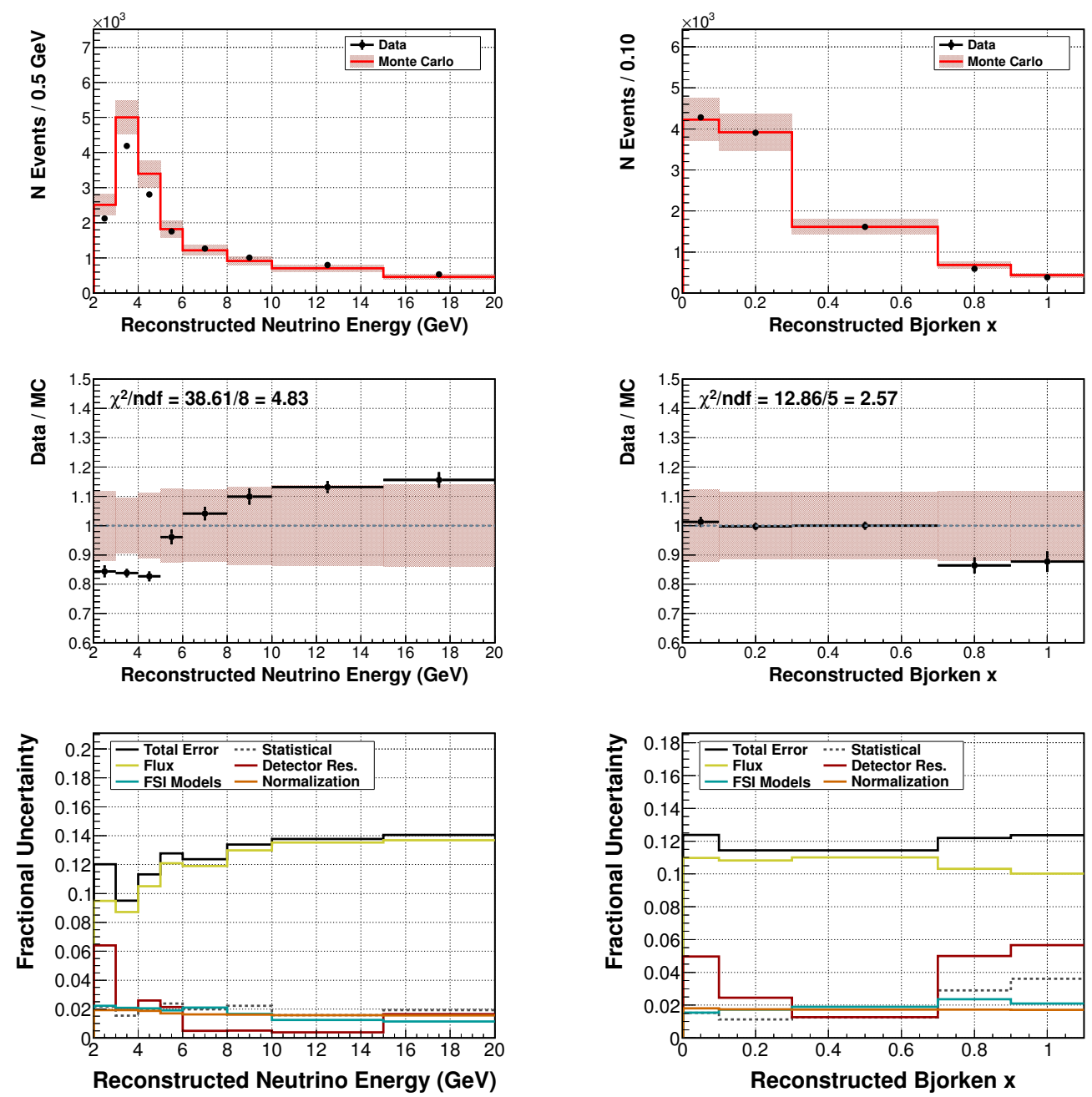

(n) Tracker modules 57-62

Figure A.9: Signal and background distribution plots. For explanation, see top of this section. 

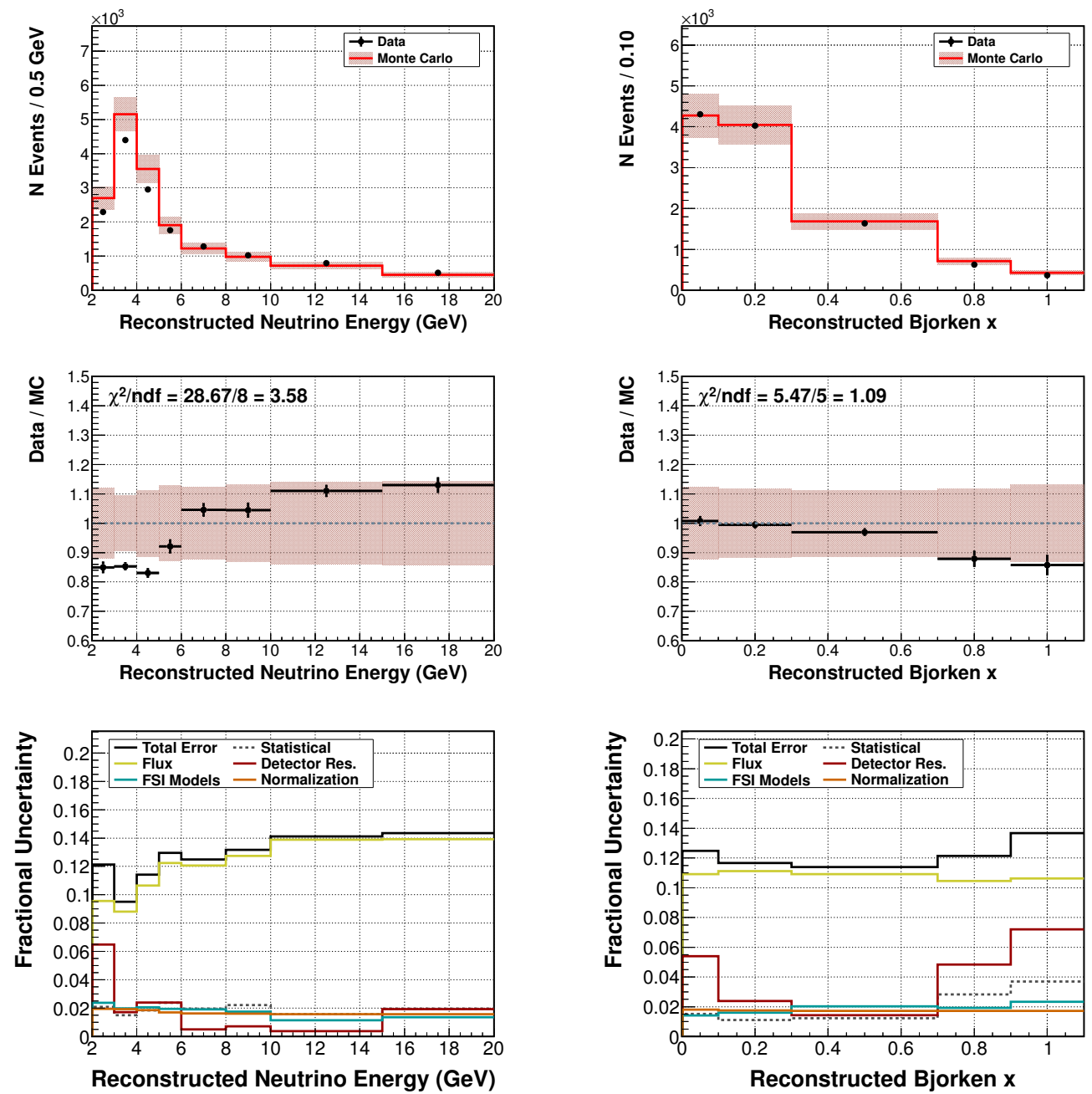

(o) Tracker modules 63-68

Figure A.9: Signal and background distribution plots. For explanation, see top of this section. 

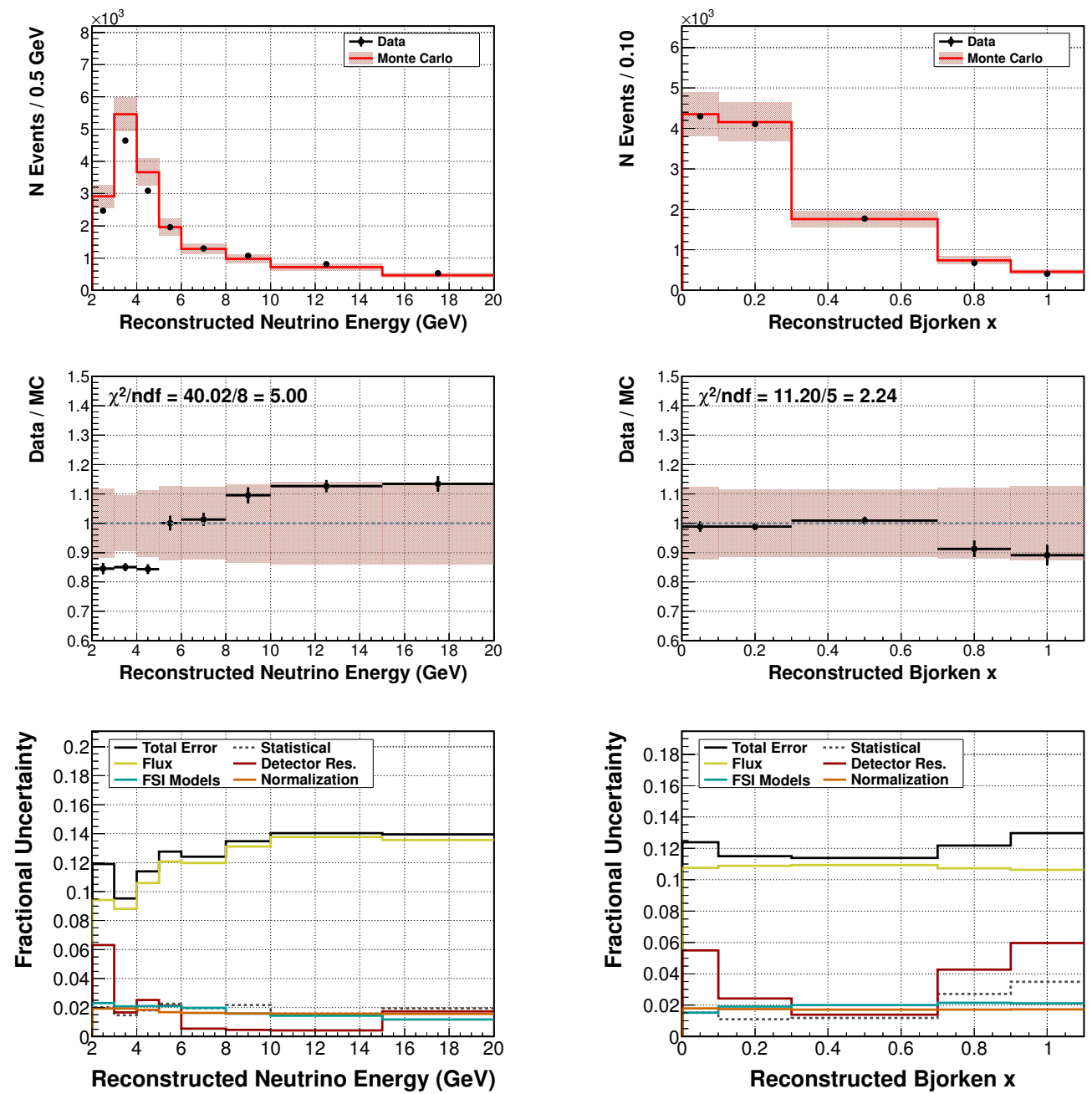

(p) Tracker modules 69-74

Figure A.9: Signal and background distribution plots. For explanation, see top of this section. 

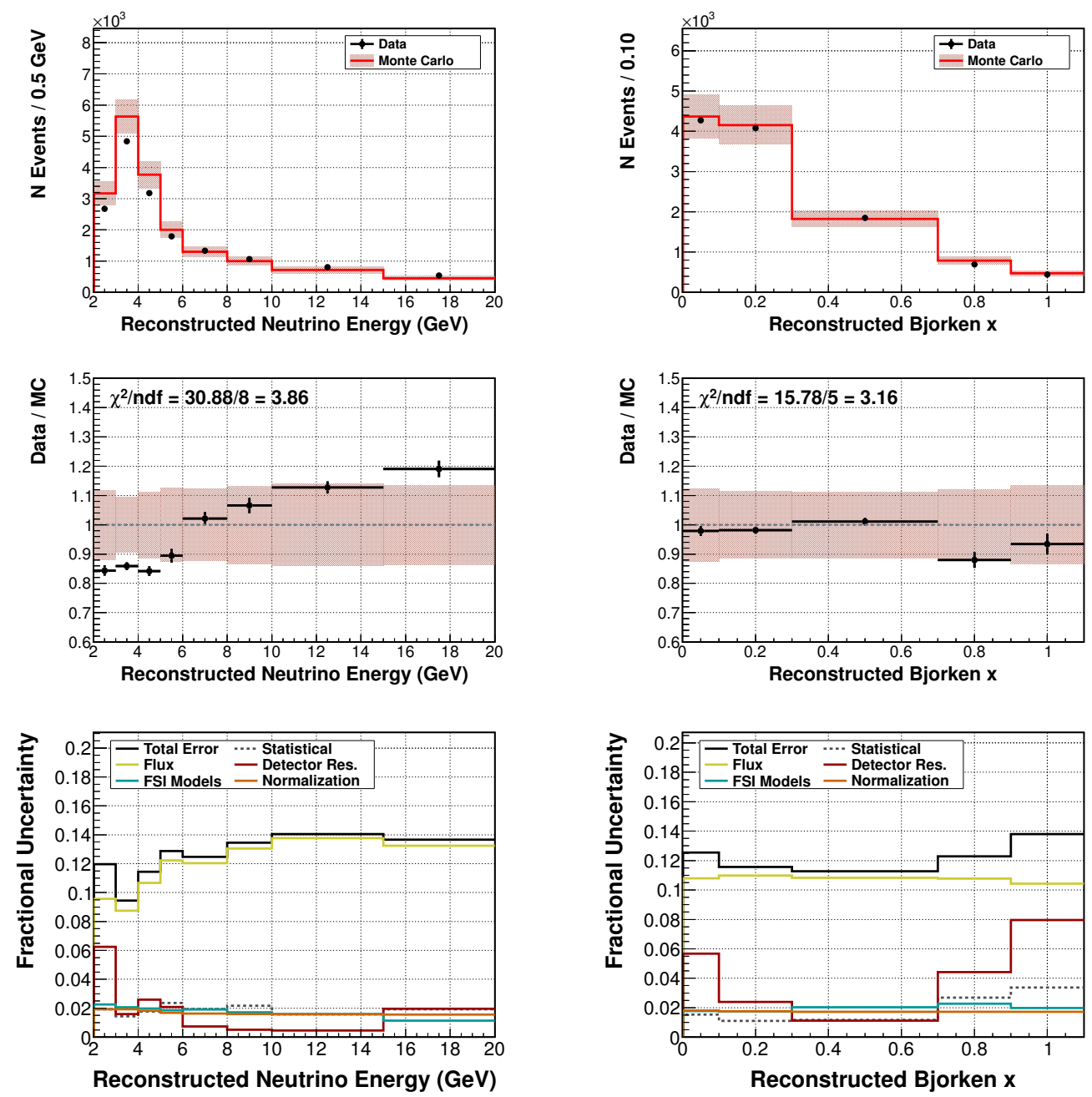

(q) Tracker modules 75-80

Figure A.9: Signal and background distribution plots. For explanation, see top of this section. 


\section{A.6 Efficiency}

The figures in this section show the efficiency and purity (measured with simulation) of each event sample. Efficiency is the fraction of generated events that are reconstructed and admitted to the proper event sample. Purity is the fraction of seleced event that were generated as signal. The major cause for impurity is scintillator contamination in passive targets. Figures on the left show the efficiency and purity. Figures on the right show the fractional errors on the efficiency. Each page shows a different event sample. 


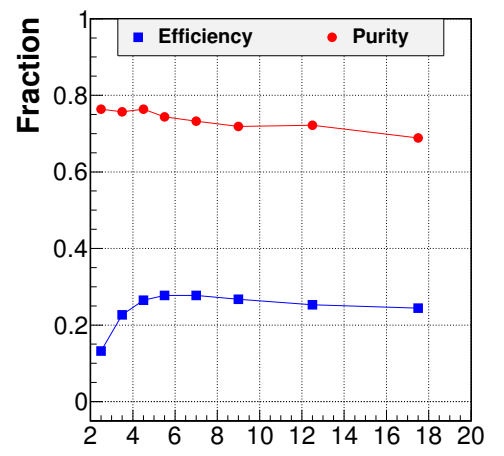

Generated Neutrino Energy (GeV)
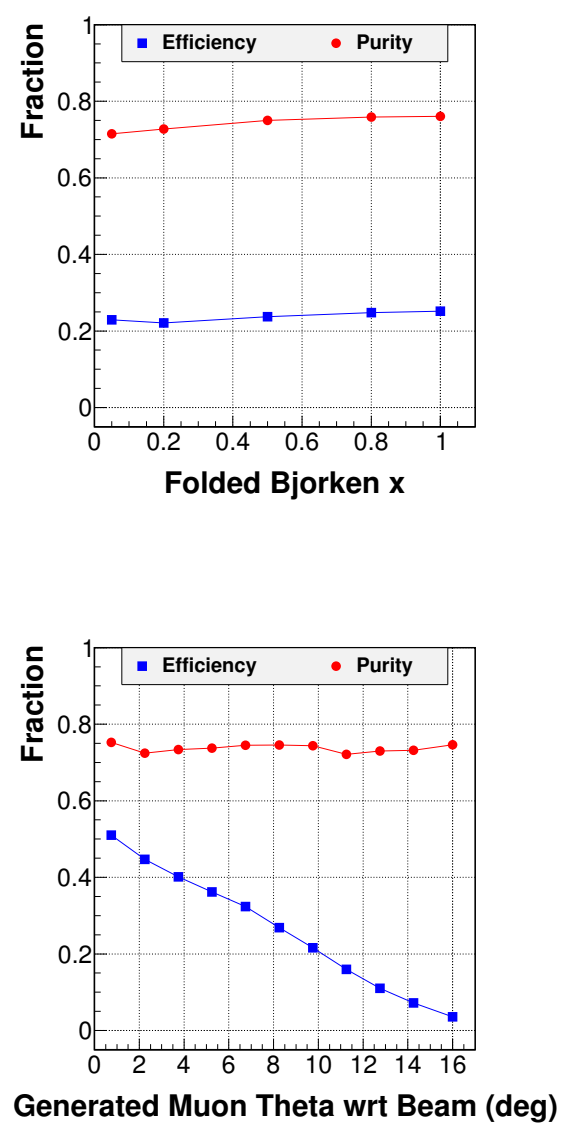
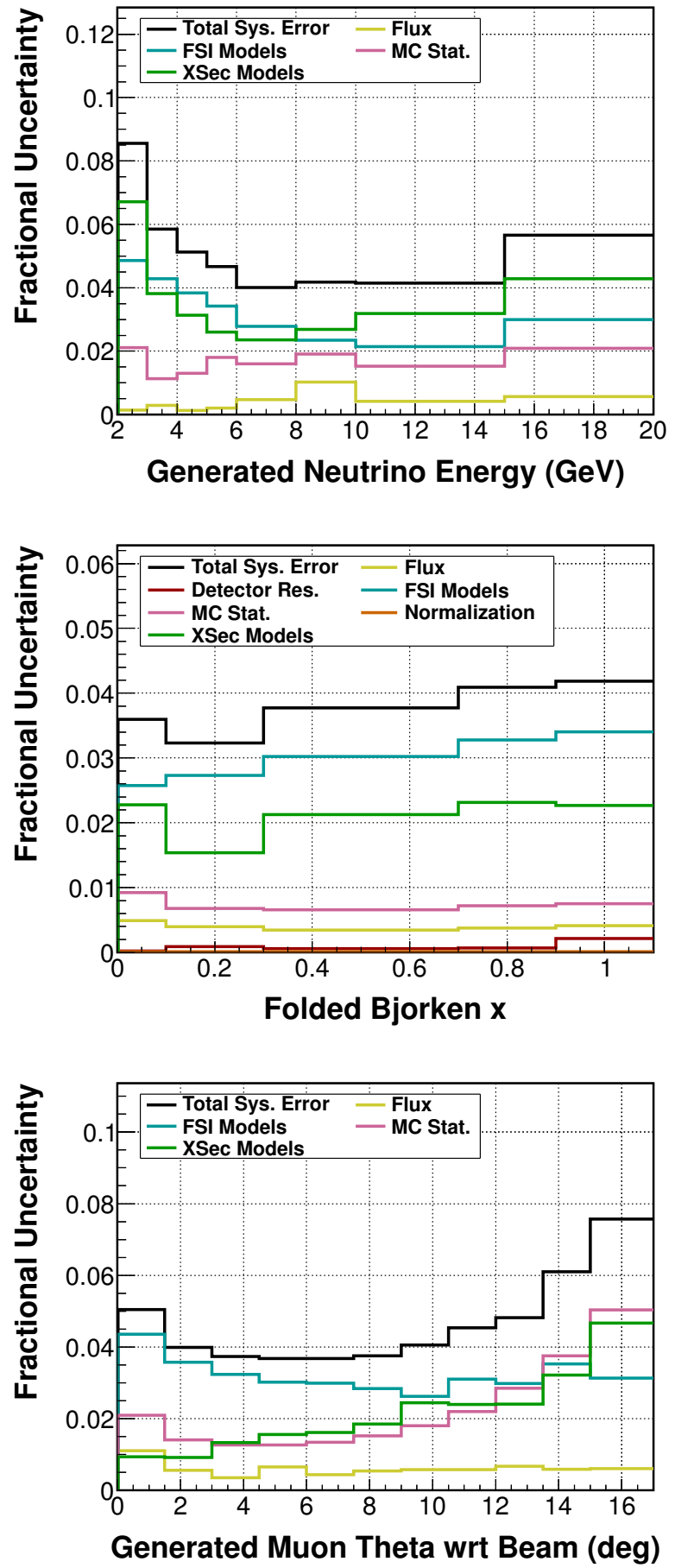

(a) Target 2 iron

Figure A.10: Efficiency and errors on efficiency. For explanation, see top of this section. 


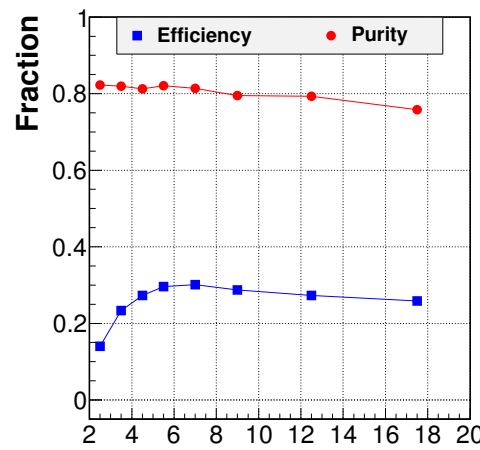

Generated Neutrino Energy $(\mathrm{GeV})$
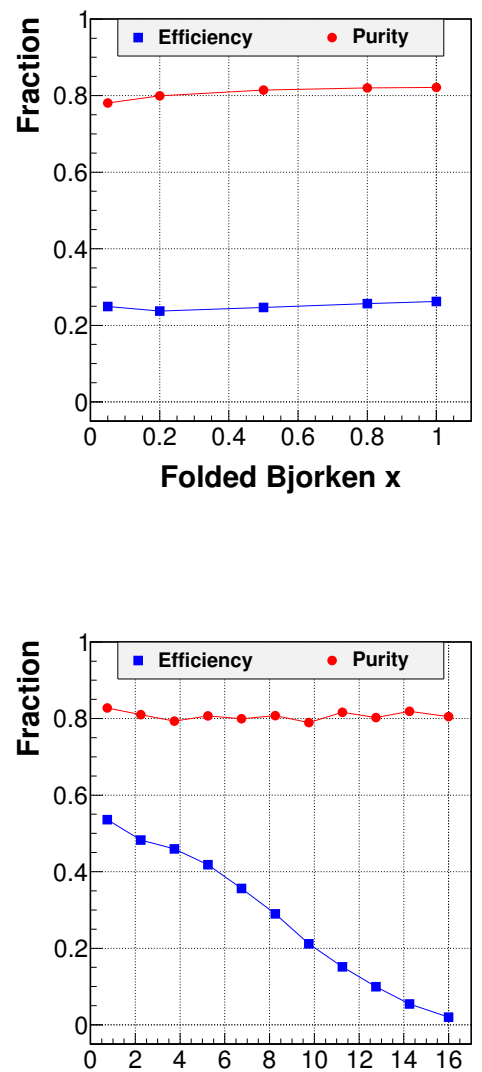

Generated Muon Theta wrt Beam (deg)

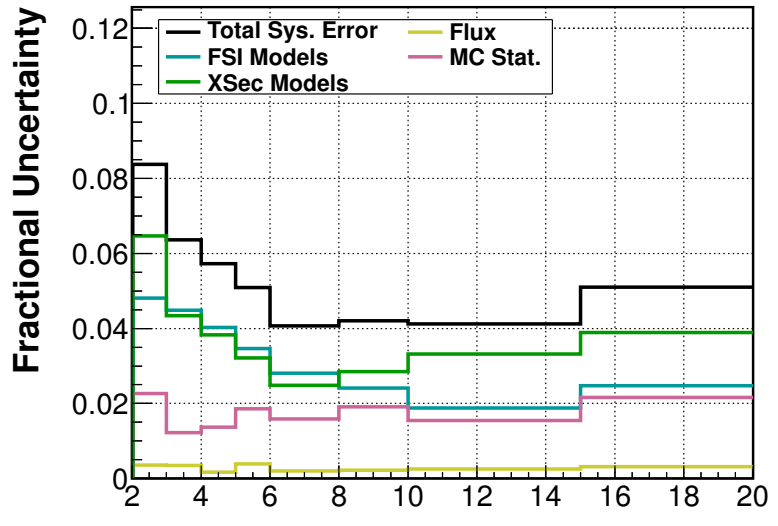

Generated Neutrino Energy (GeV)
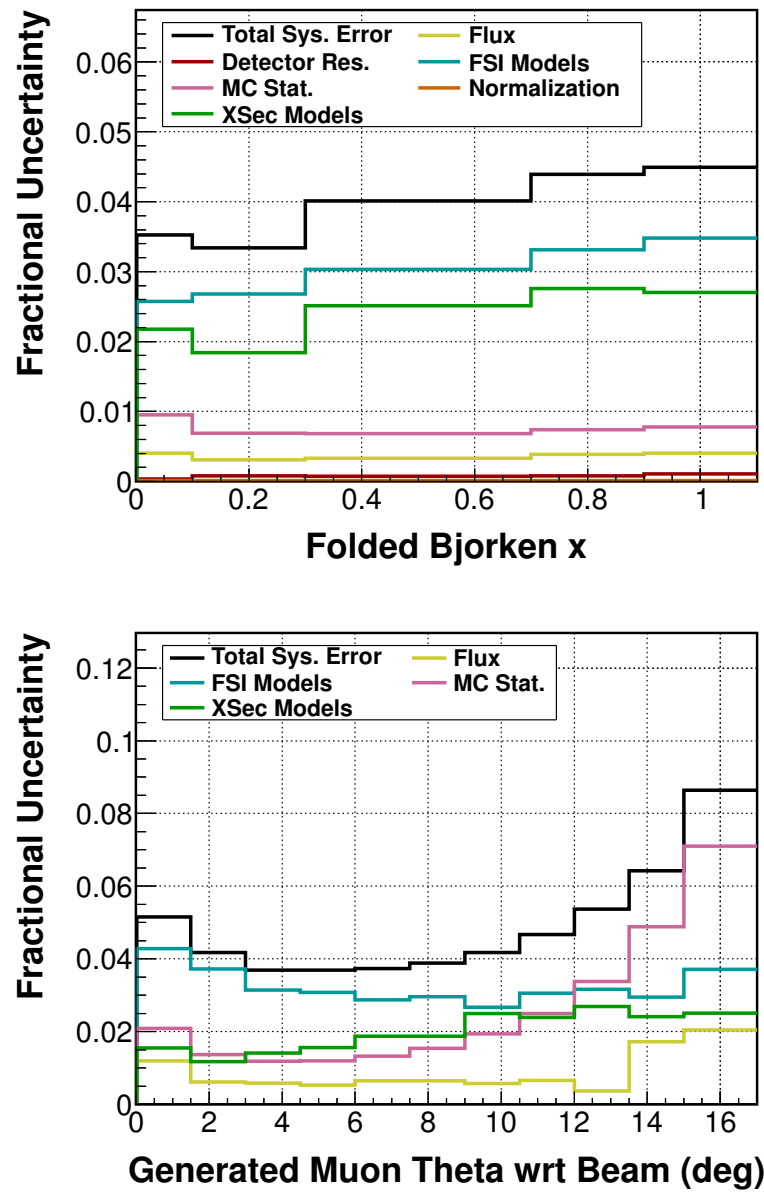

(b) Target 2 lead

Figure A.10: Efficiency and errors on efficiency. For explanation, see top of this section. 


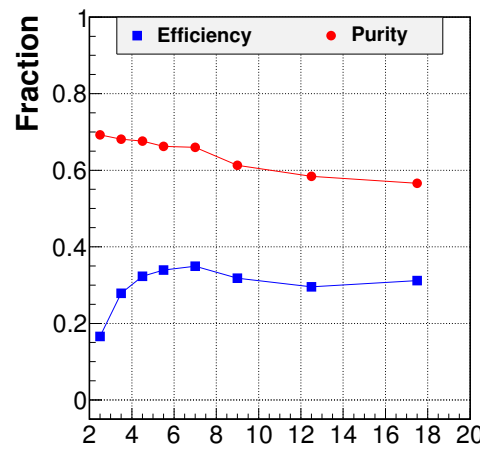

Generated Neutrino Energy $(\mathrm{GeV})$
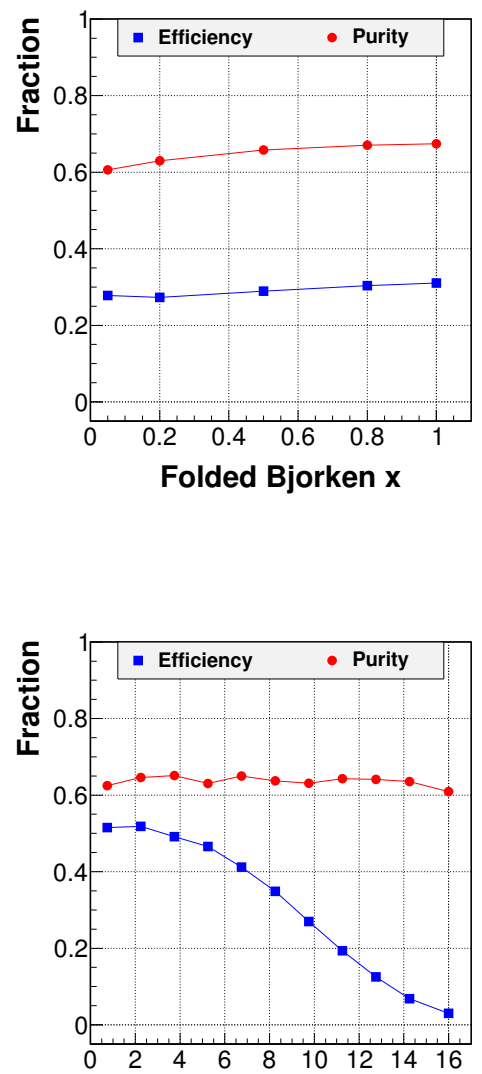

Generated Muon Theta wrt Beam (deg)

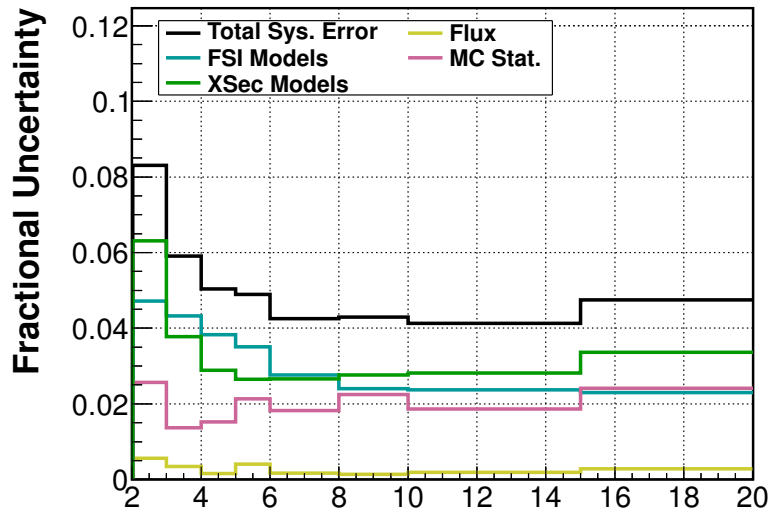

Generated Neutrino Energy (GeV)

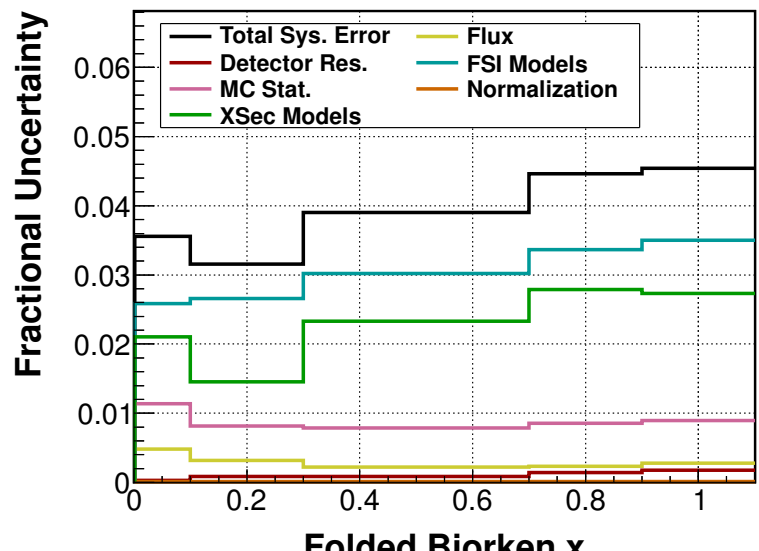

Folded Bjorken x

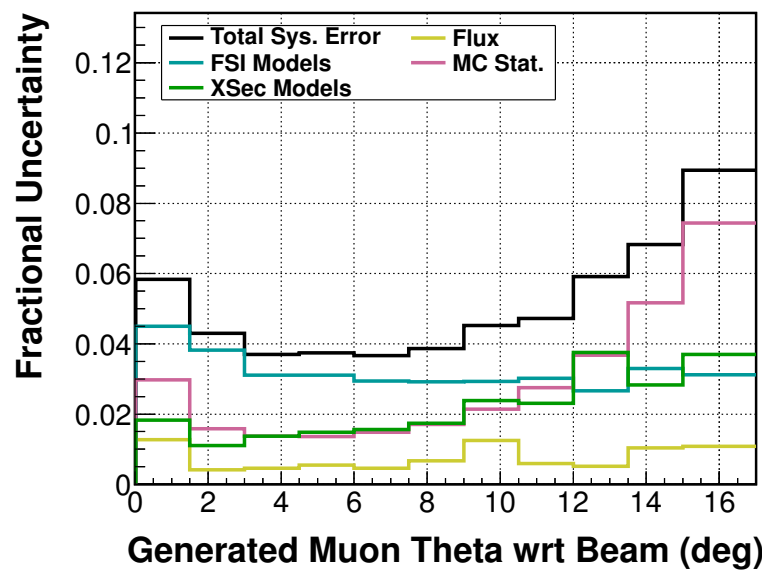

(c) Target 3 carbon

Figure A.10: Efficiency and errors on efficiency. For explanation, see top of this section. 


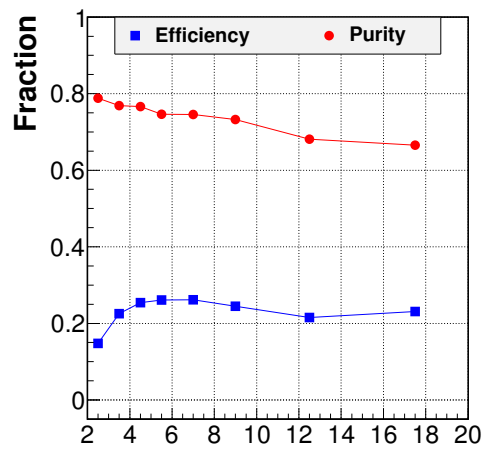

Generated Neutrino Energy (GeV)
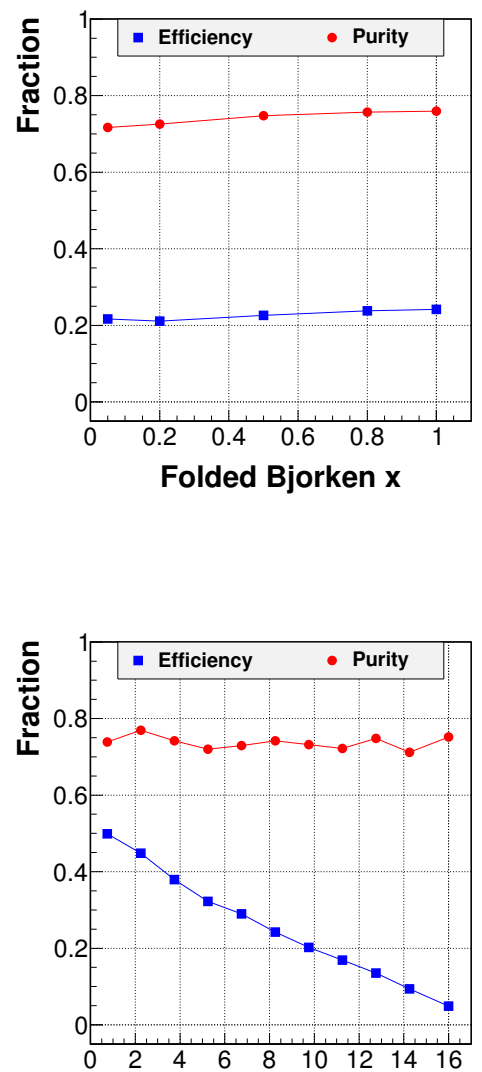

Generated Muon Theta wrt Beam (deg)

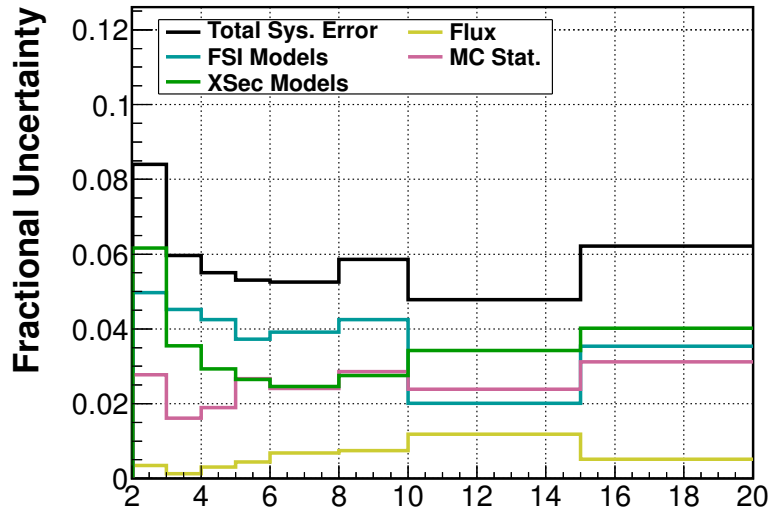

Generated Neutrino Energy (GeV)
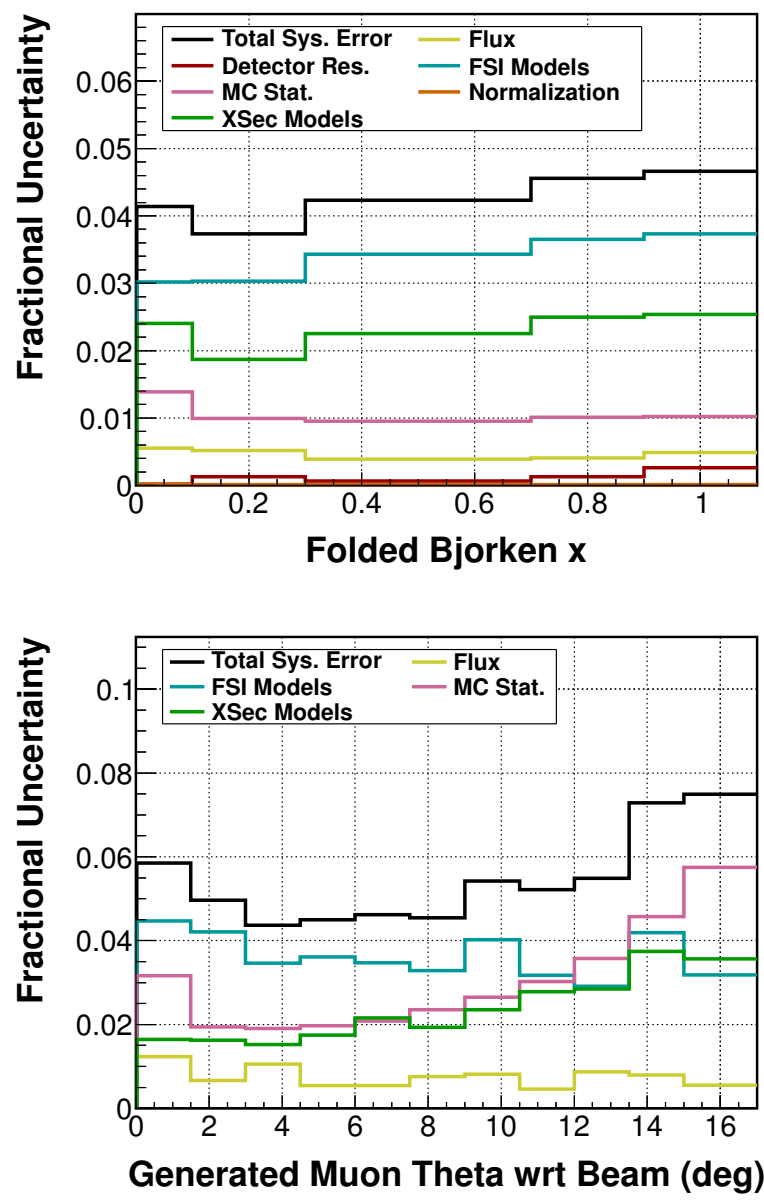

(d) Target 3 iron

Figure A.10: Efficiency and errors on efficiency. For explanation, see top of this section. 


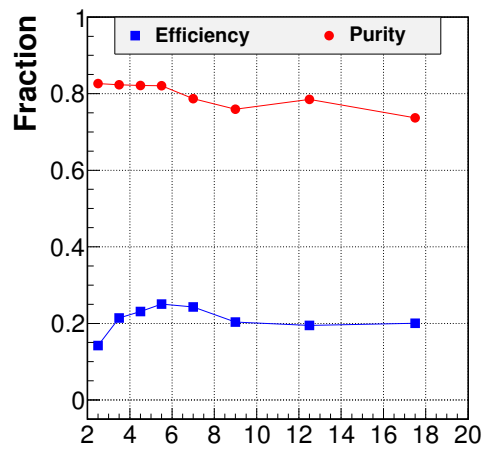

Generated Neutrino Energy (GeV)
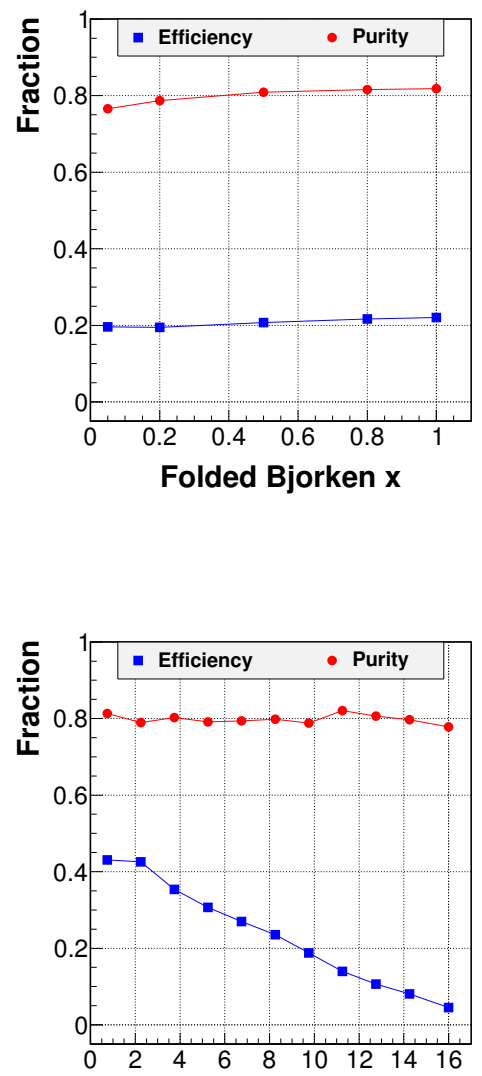

Generated Muon Theta wrt Beam (deg)

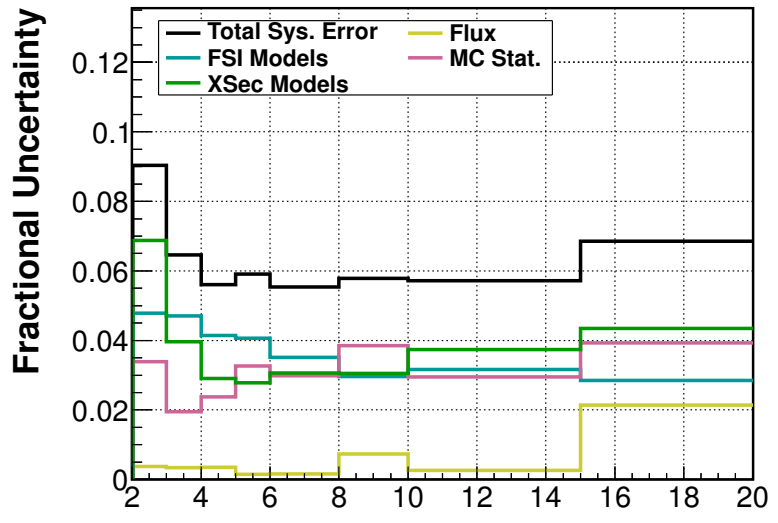

Generated Neutrino Energy (GeV)

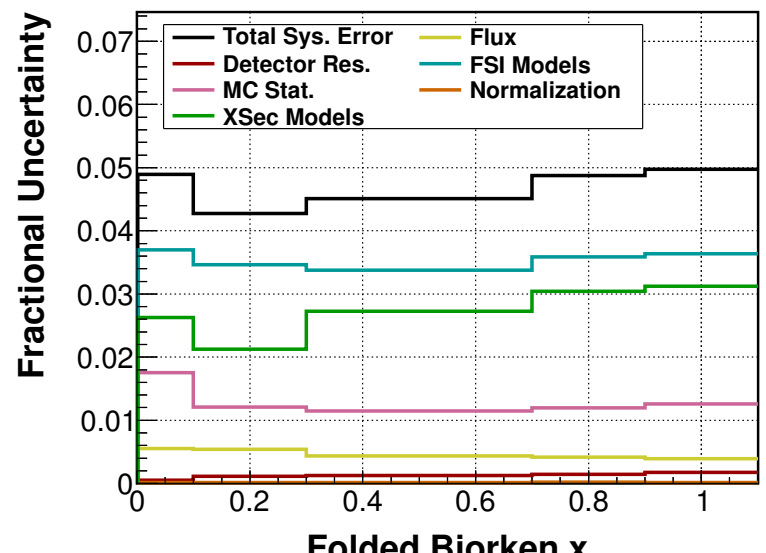

Folded Bjorken x

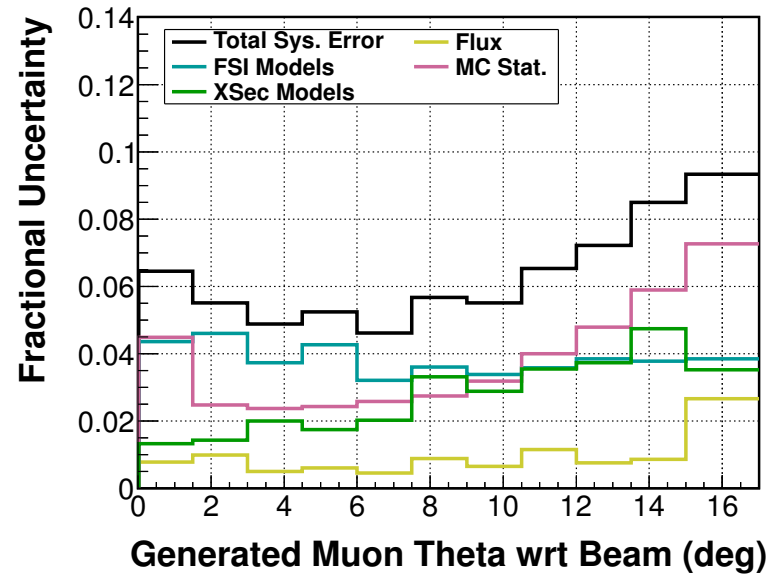

(e) Target 3 lead

Figure A.10: Efficiency and errors on efficiency. For explanation, see top of this section. 


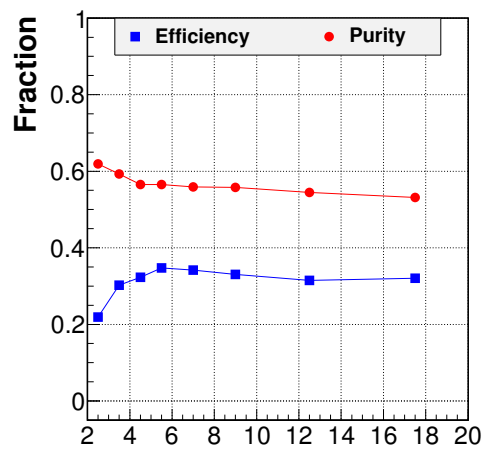

Generated Neutrino Energy (GeV)
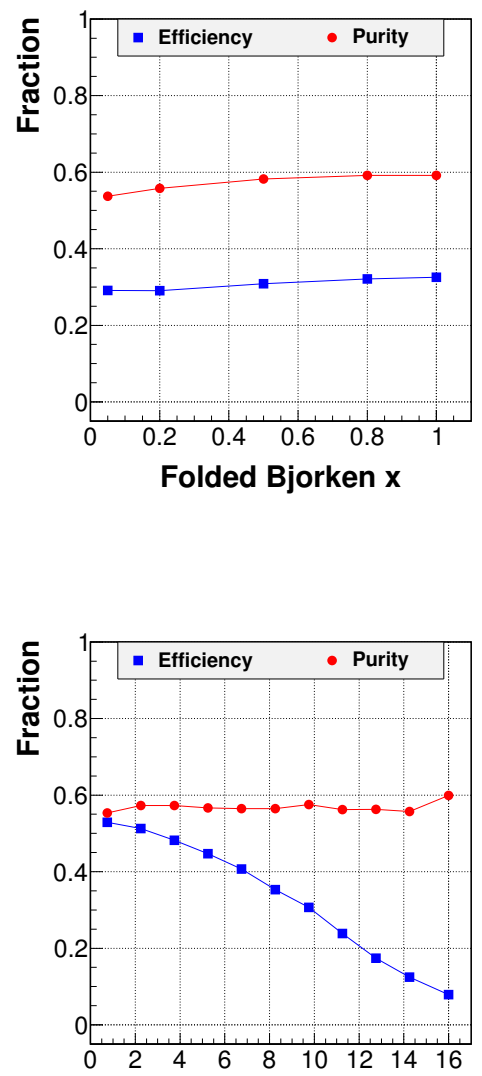

Generated Muon Theta wrt Beam (deg)
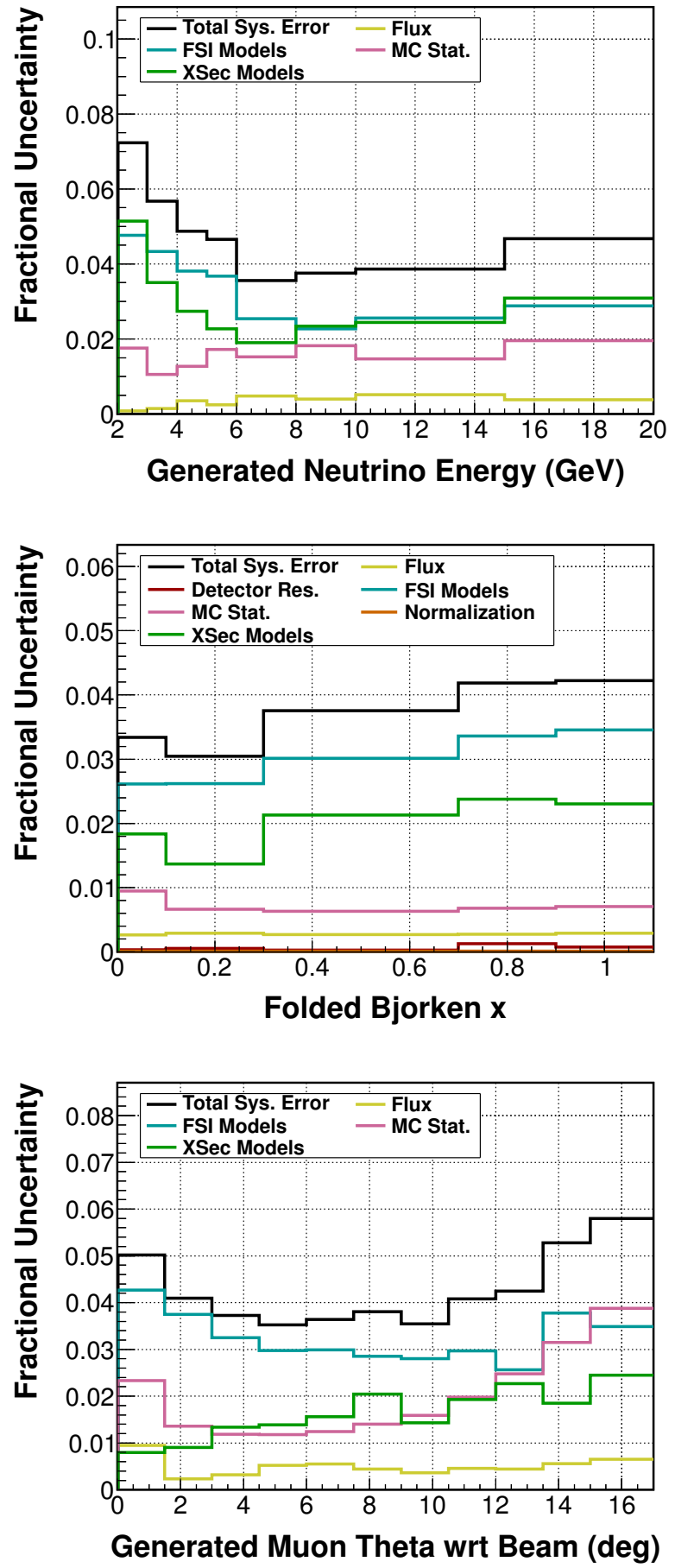

(f) Target 4 lead

Figure A.10: Efficiency and errors on efficiency. For explanation, see top of this section. 


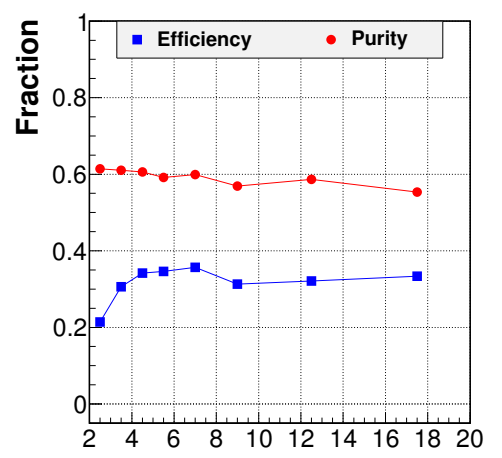

Generated Neutrino Energy (GeV)
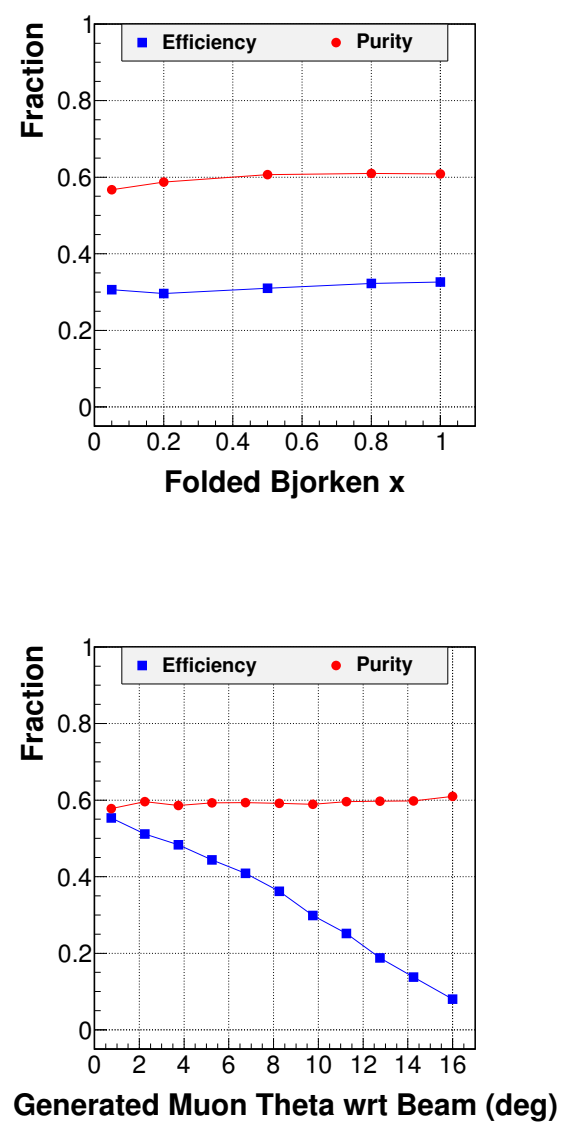
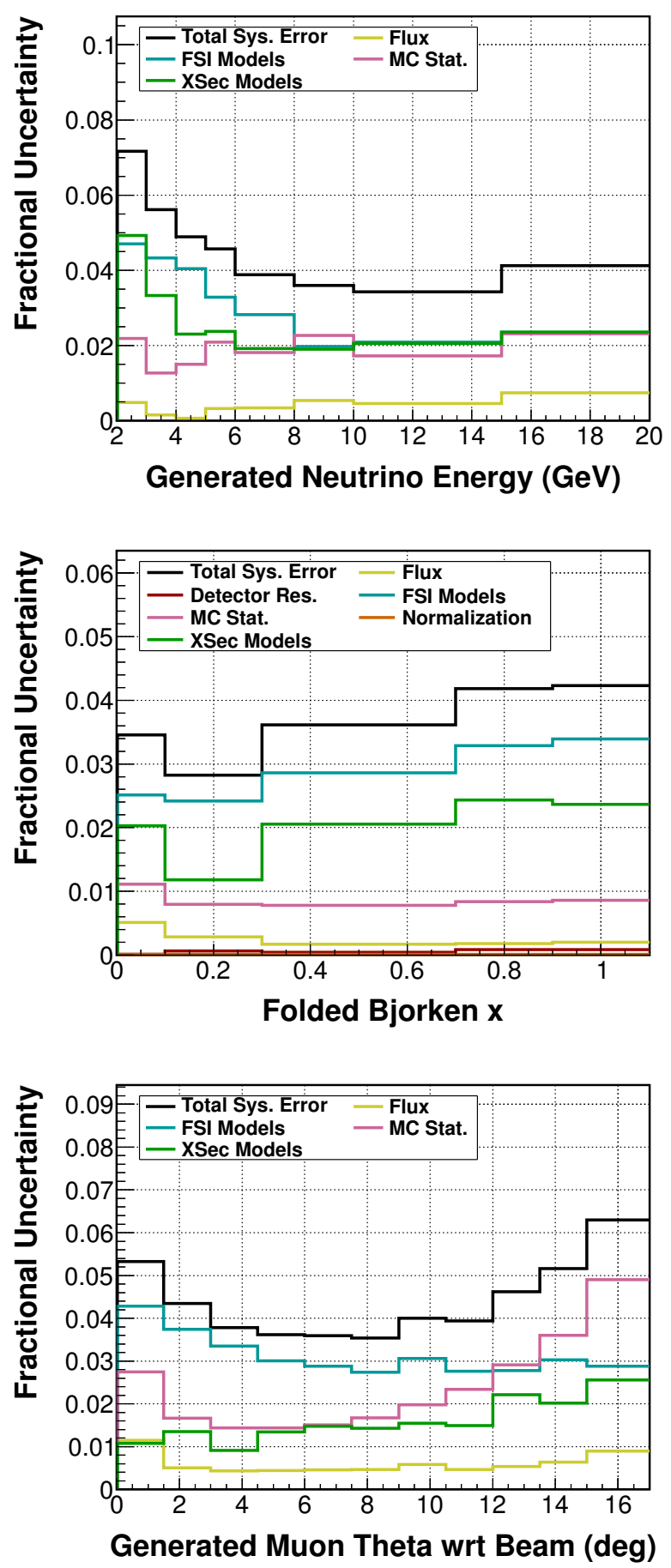

(g) Target 5 iron

Figure A.10: Efficiency and errors on efficiency. For explanation, see top of this section. 


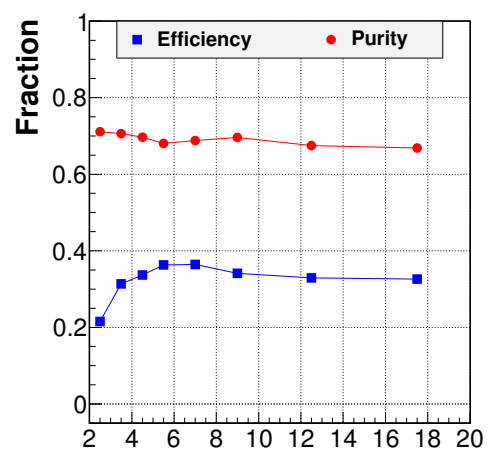

Generated Neutrino Energy (GeV)
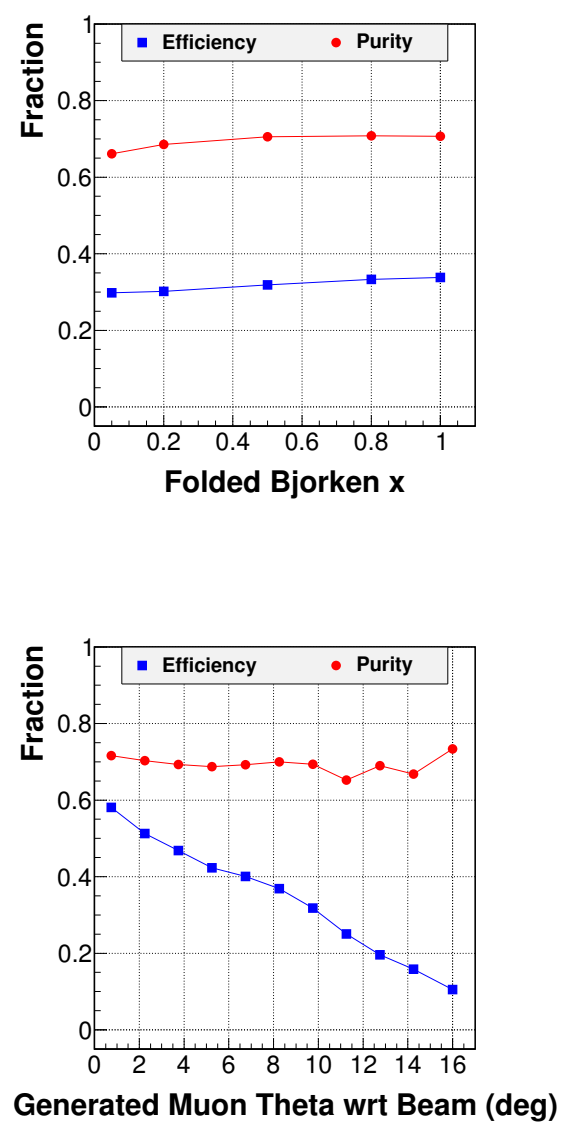

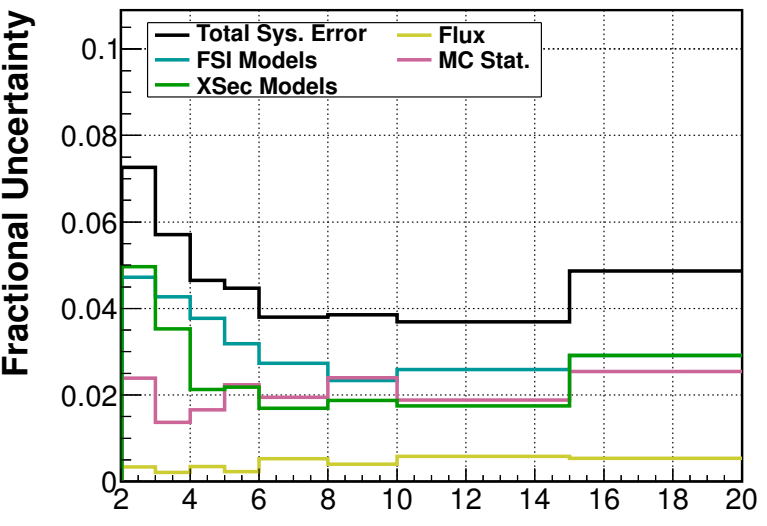

Generated Neutrino Energy (GeV)
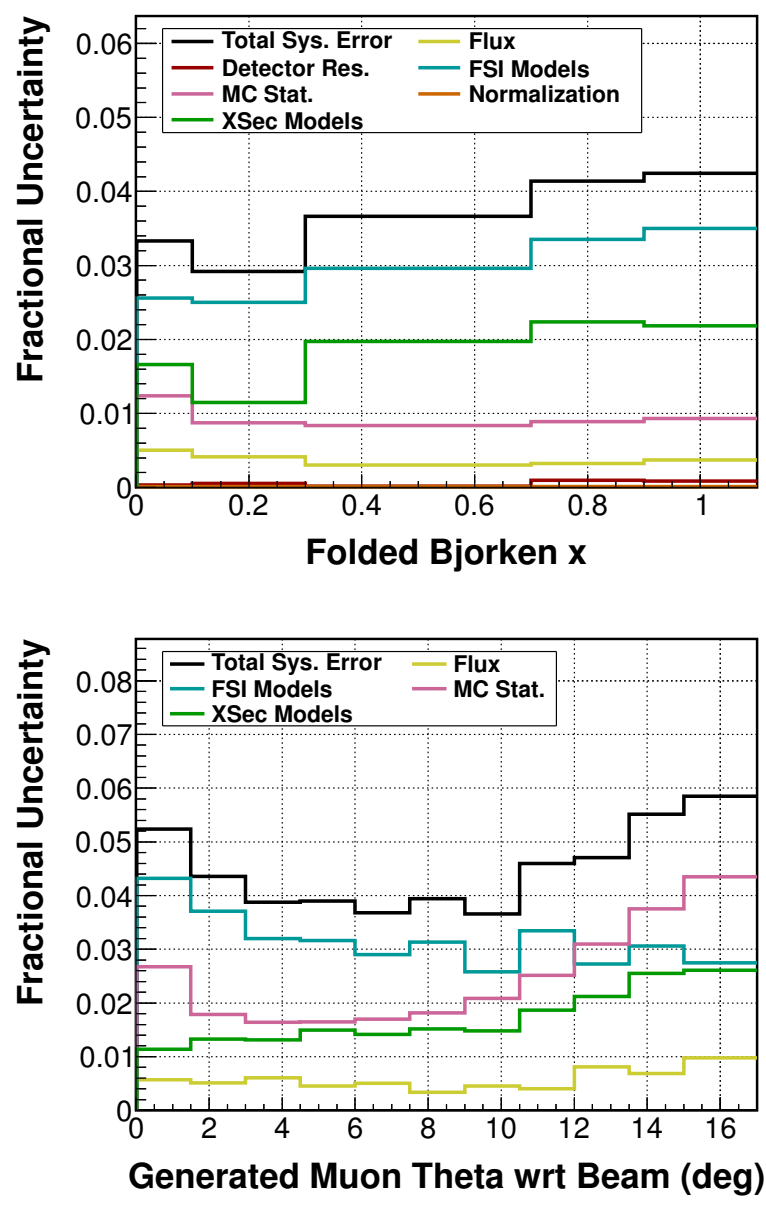

(h) Target 5 lead

Figure A.10: Efficiency and errors on efficiency. For explanation, see top of this section. 


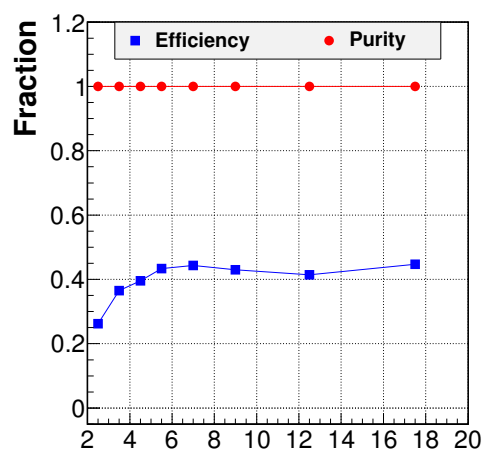

Generated Neutrino Energy (GeV)
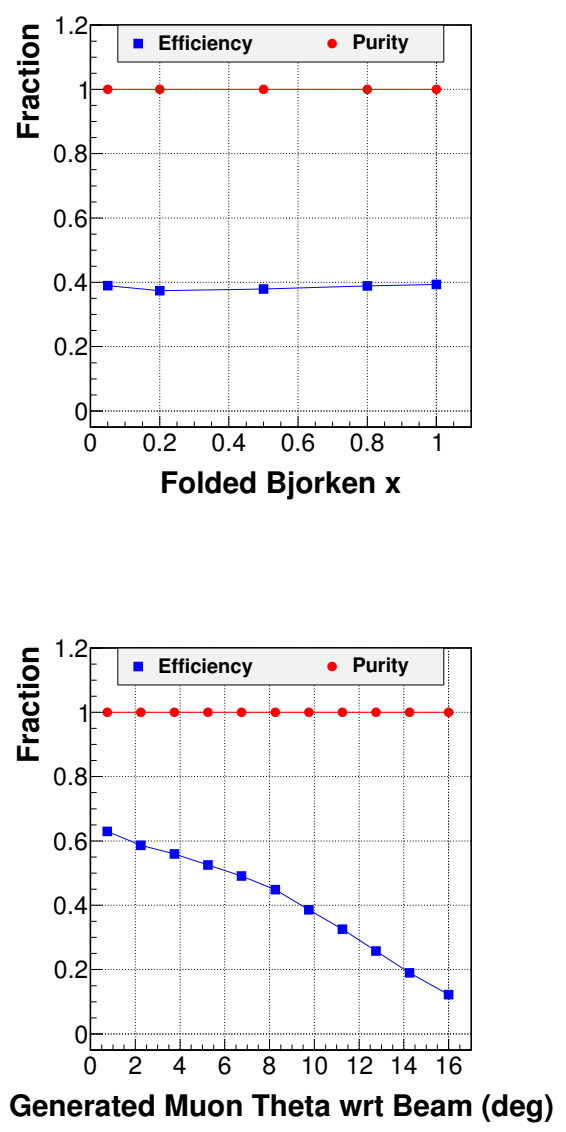
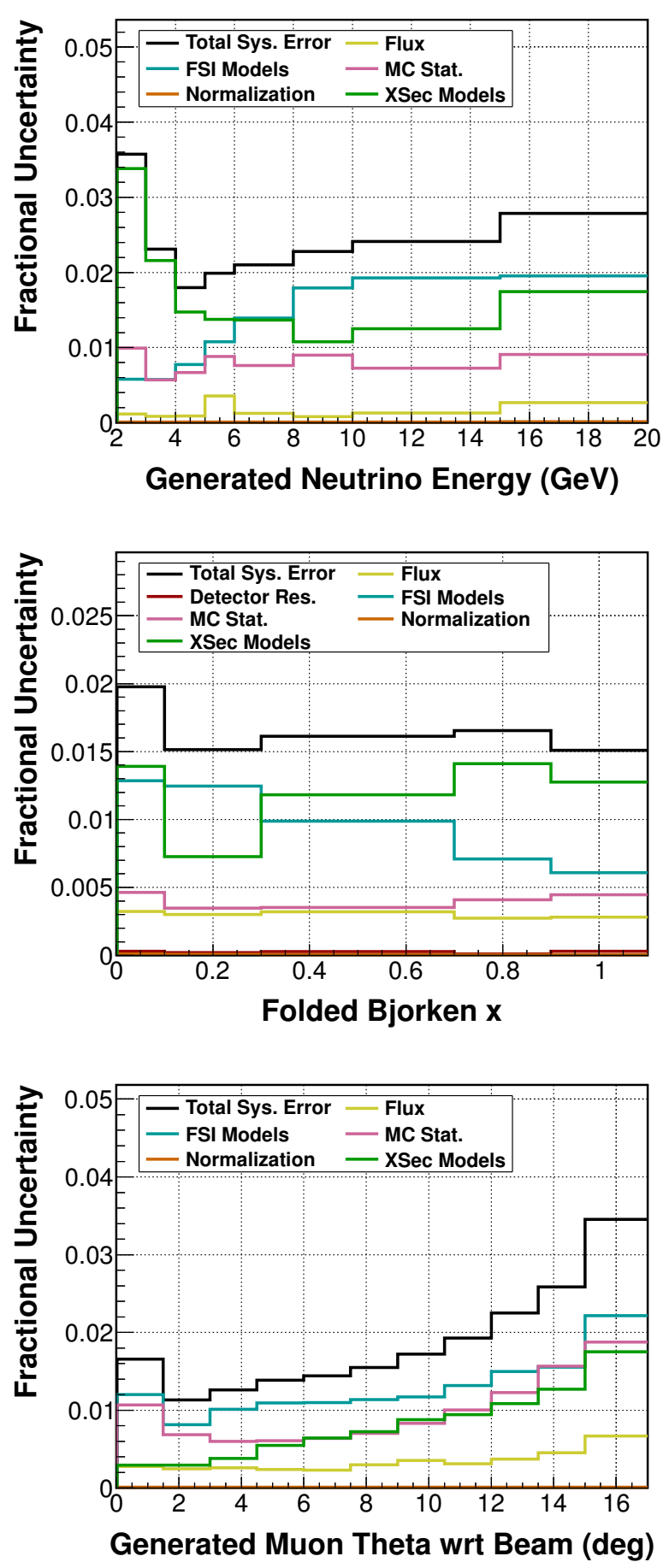

(i) Tracker modules 27-32

Figure A.10: Efficiency and errors on efficiency. For explanation, see top of this section. 


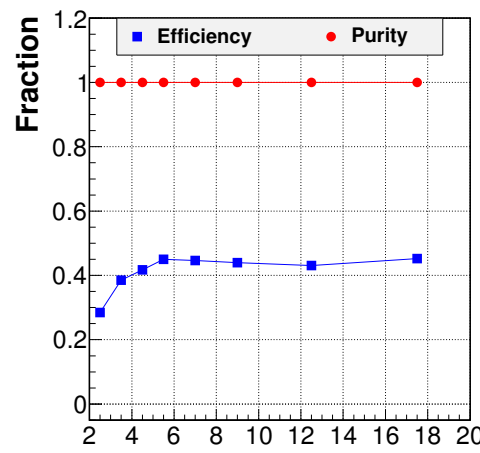

Generated Neutrino Energy (GeV)
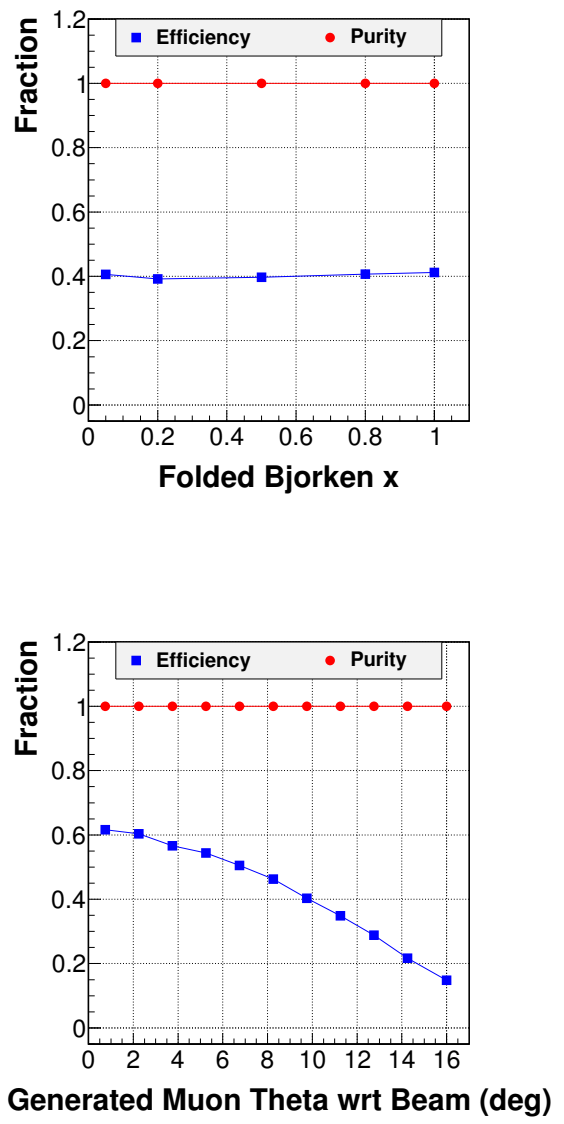
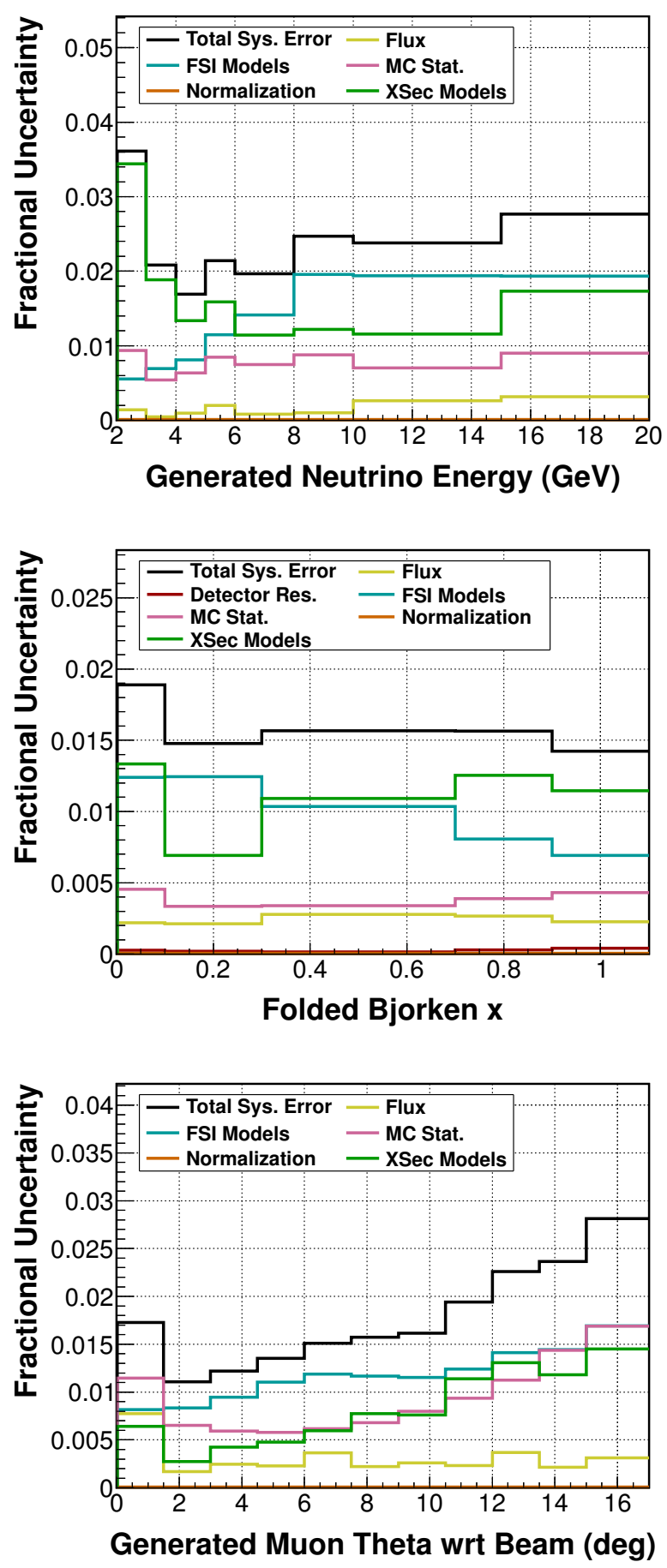

(j) Tracker modules 33-38

Figure A.10: Efficiency and errors on efficiency. For explanation, see top of this section. 


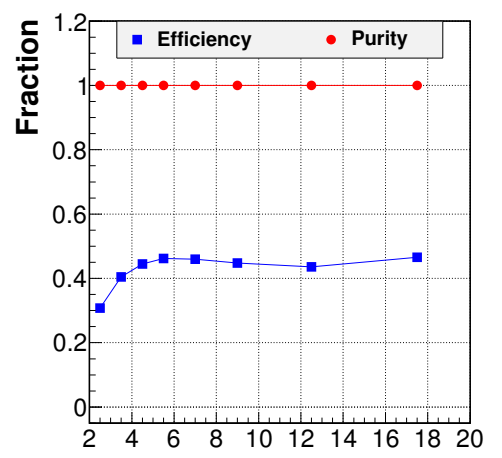

Generated Neutrino Energy (GeV)
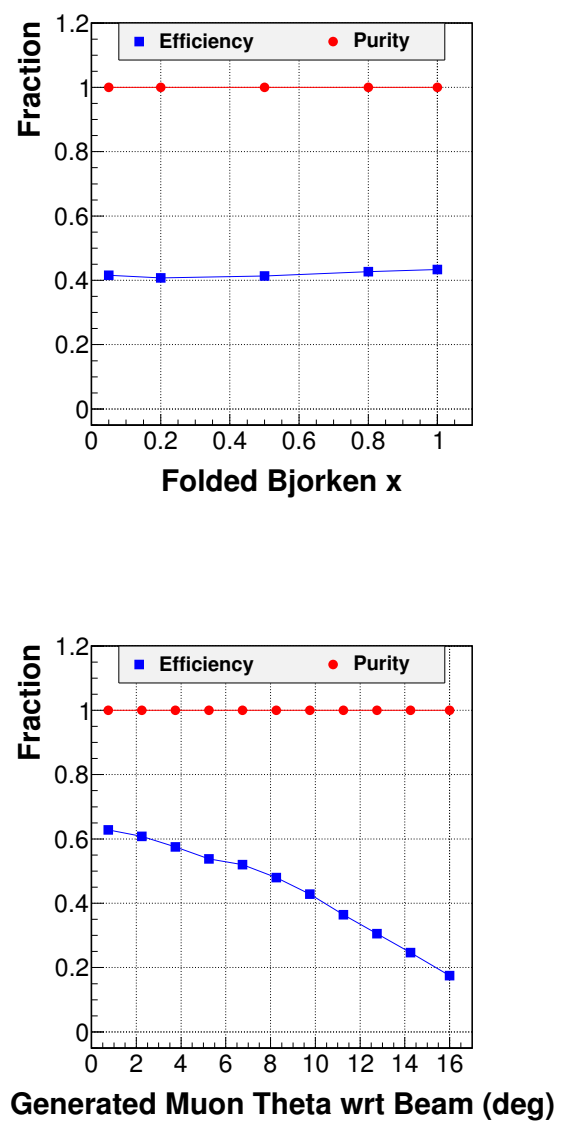
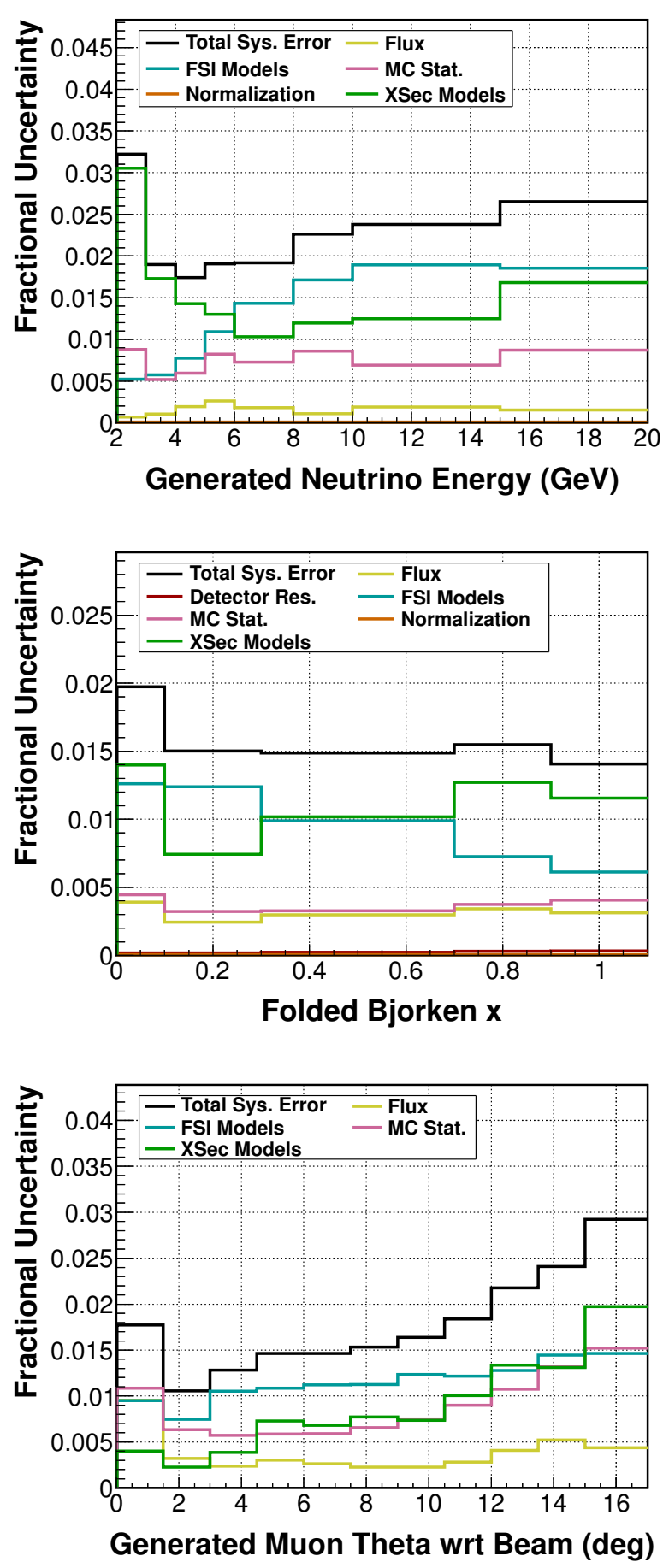

(k) Tracker modules 39-44

Figure A.10: Efficiency and errors on efficiency. For explanation, see top of this section. 


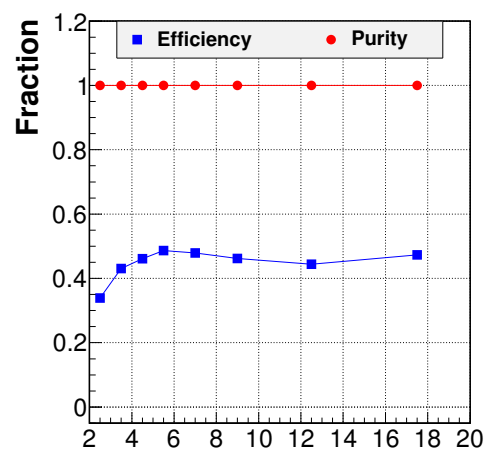

Generated Neutrino Energy (GeV)
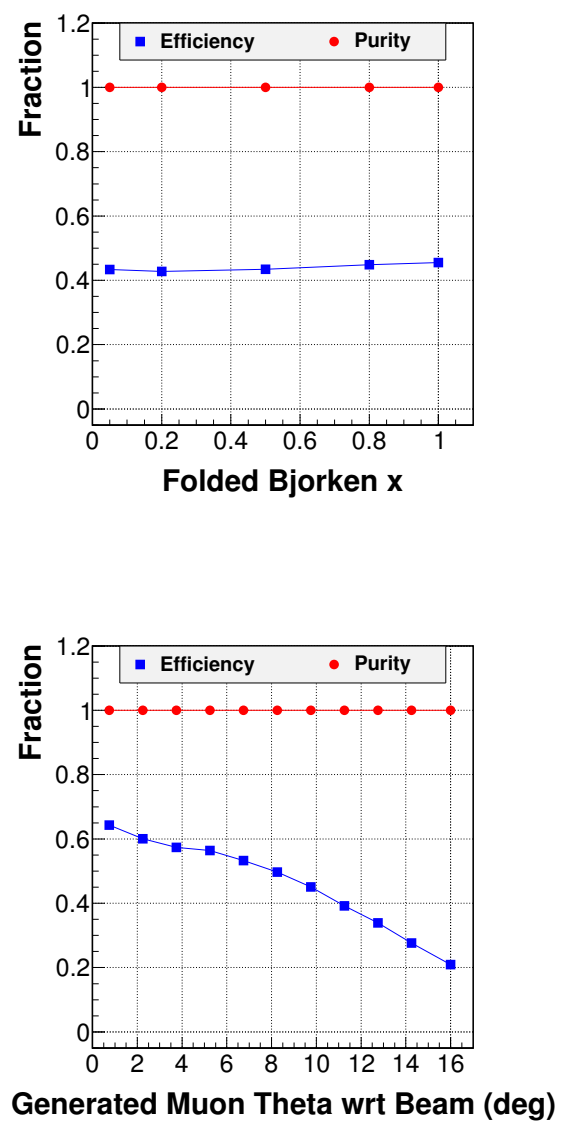
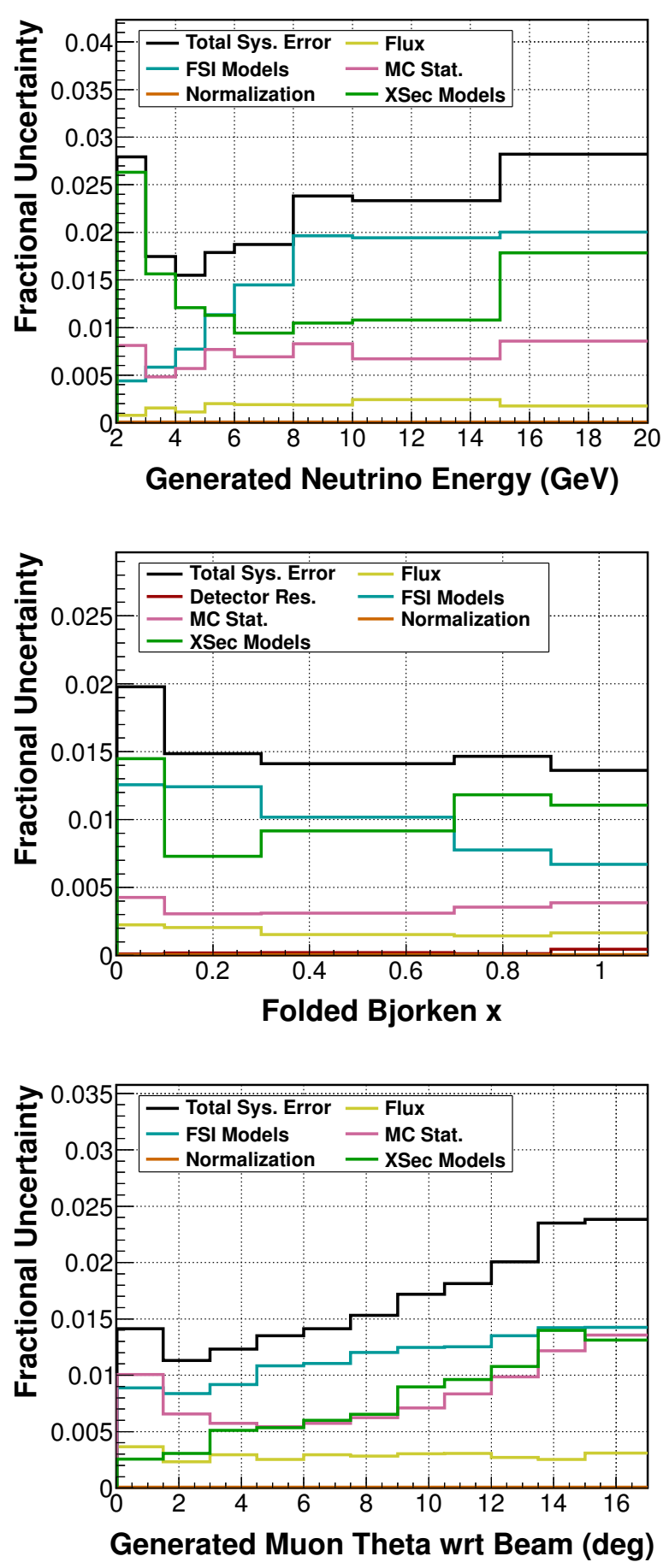

(1) Tracker modules $45-50$

Figure A.10: Efficiency and errors on efficiency. For explanation, see top of this section. 


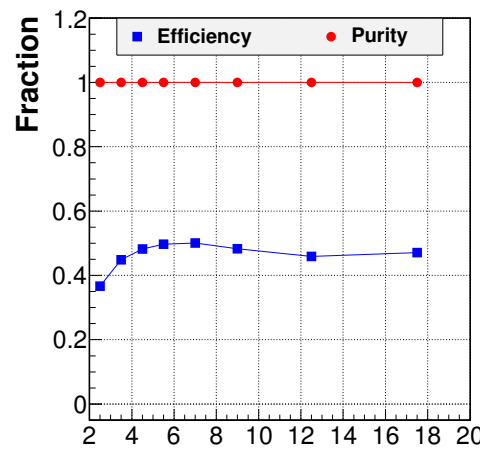

Generated Neutrino Energy (GeV)
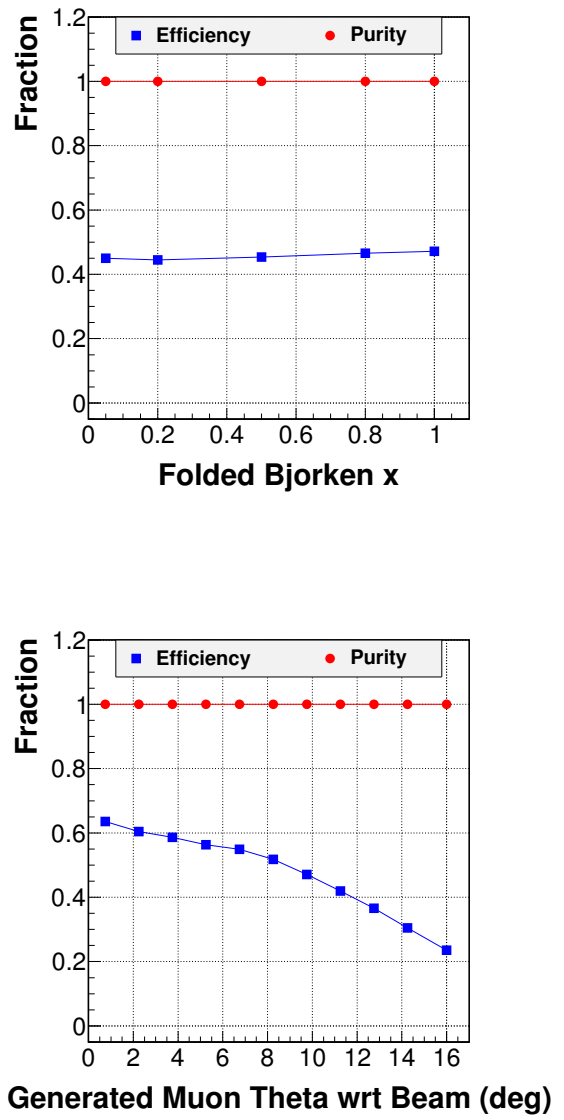
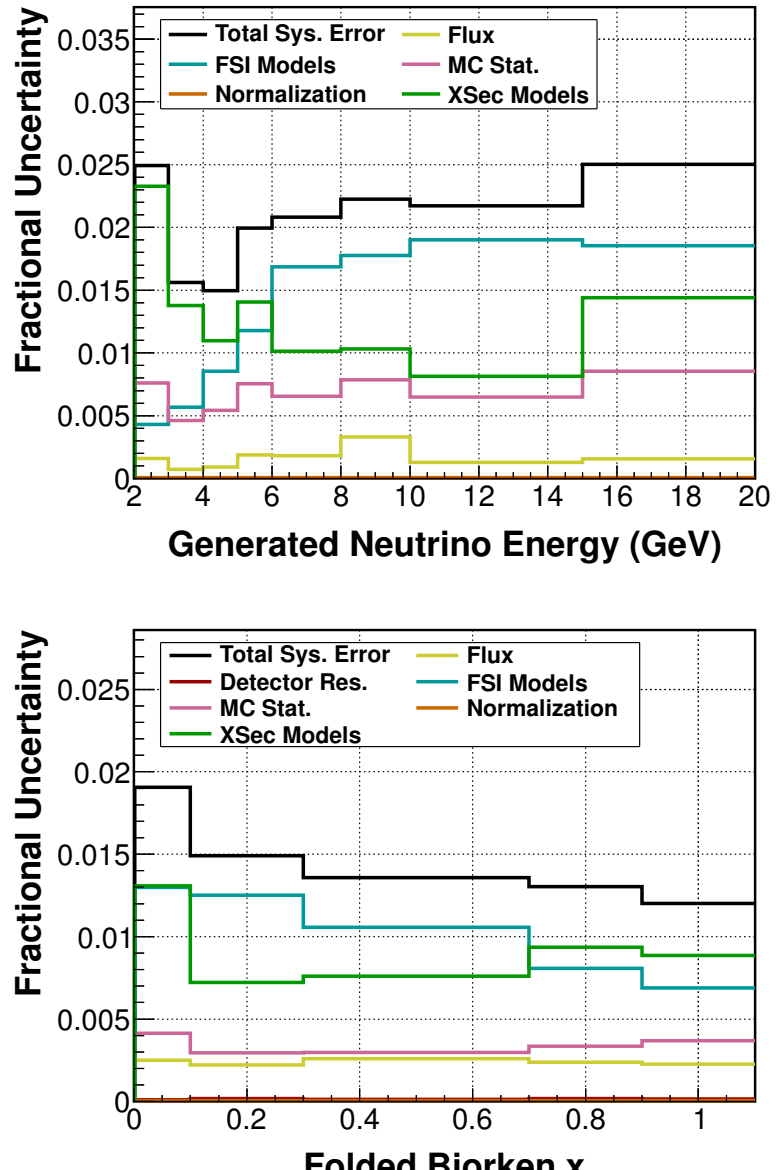

Folded Bjorken x

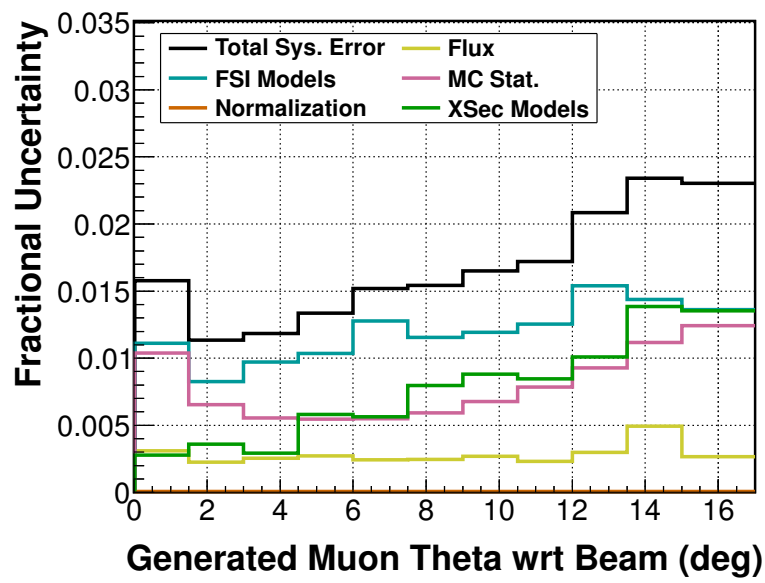

(m) Tracker modules 51-56

Figure A.10: Efficiency and errors on efficiency. For explanation, see top of this section. 


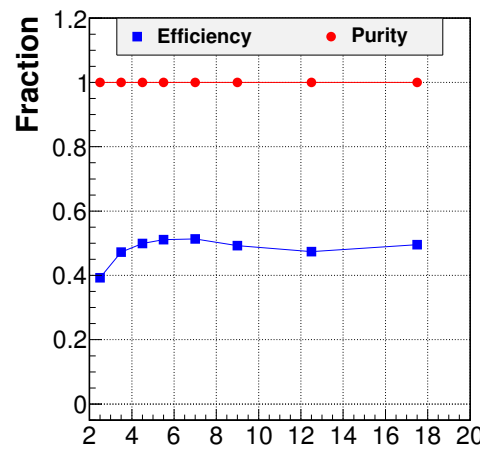

Generated Neutrino Energy (GeV)
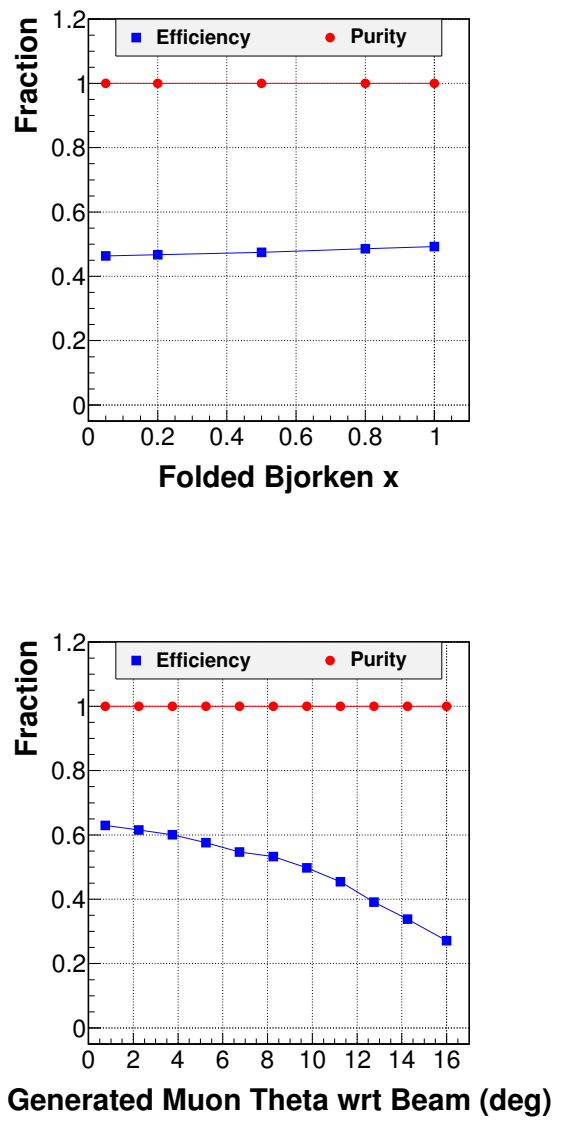

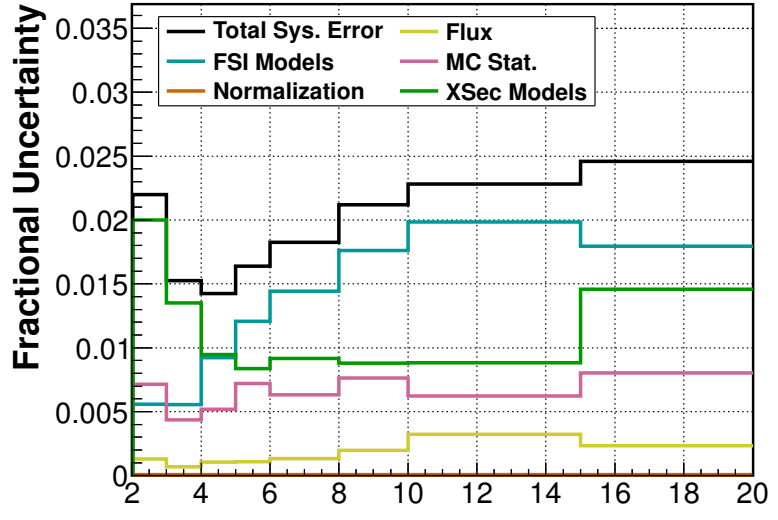

Generated Neutrino Energy (GeV)

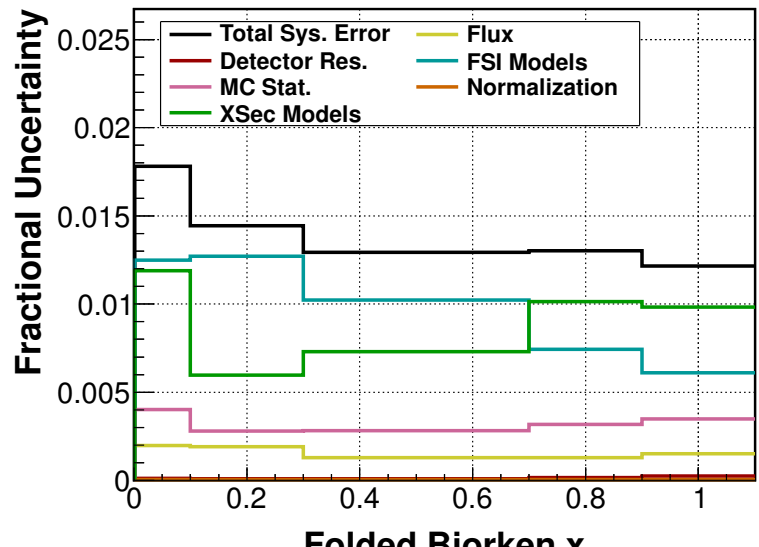

Folded Bjorken x

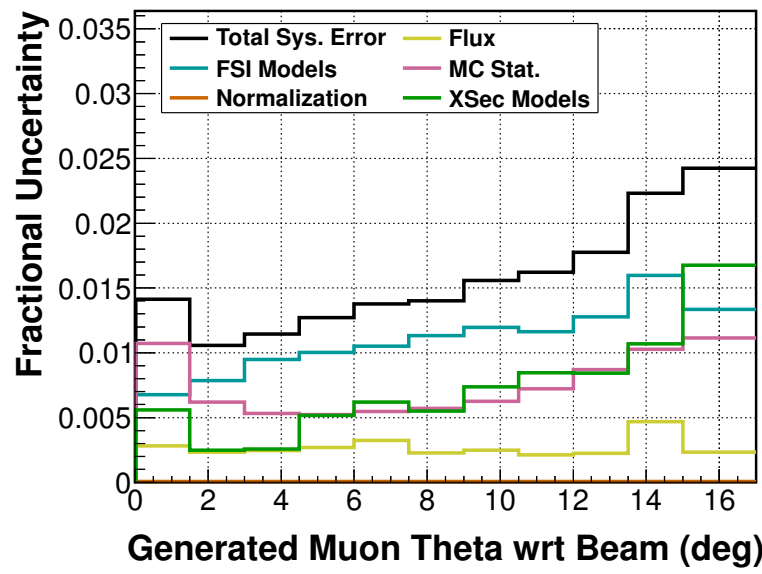

(n) Tracker modules 57-62

Figure A.10: Efficiency and errors on efficiency. For explanation, see top of this section. 


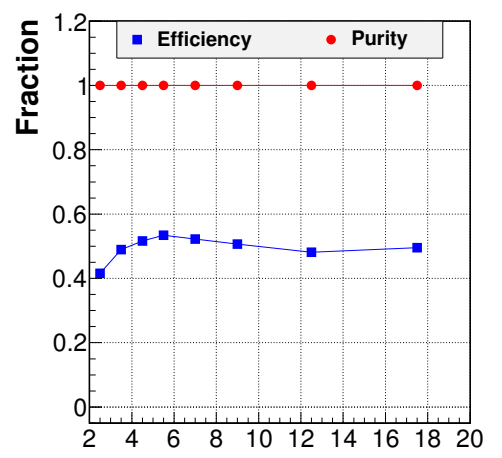

Generated Neutrino Energy (GeV)
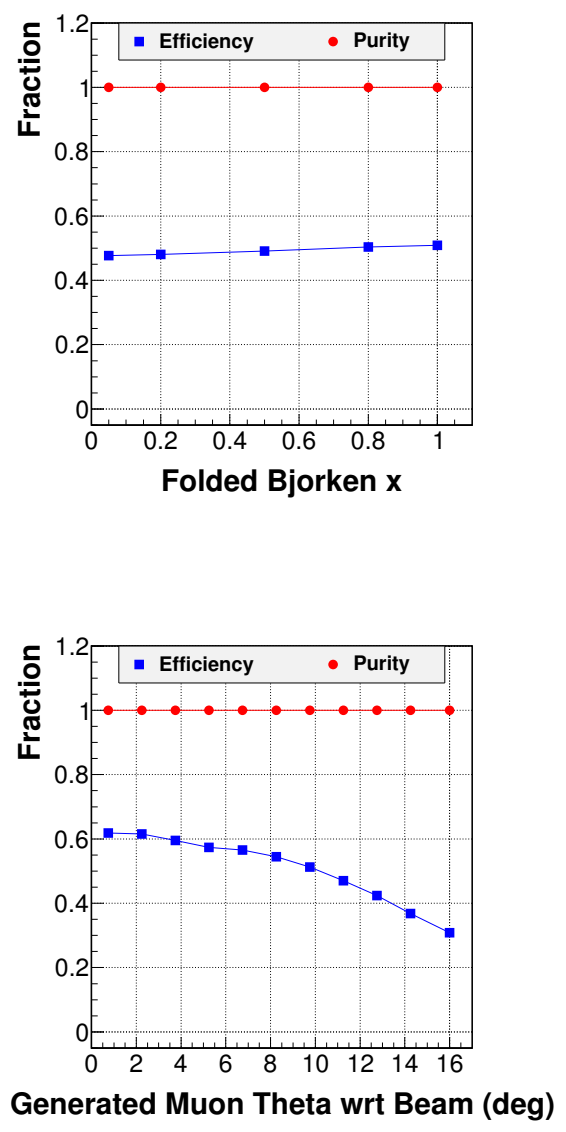
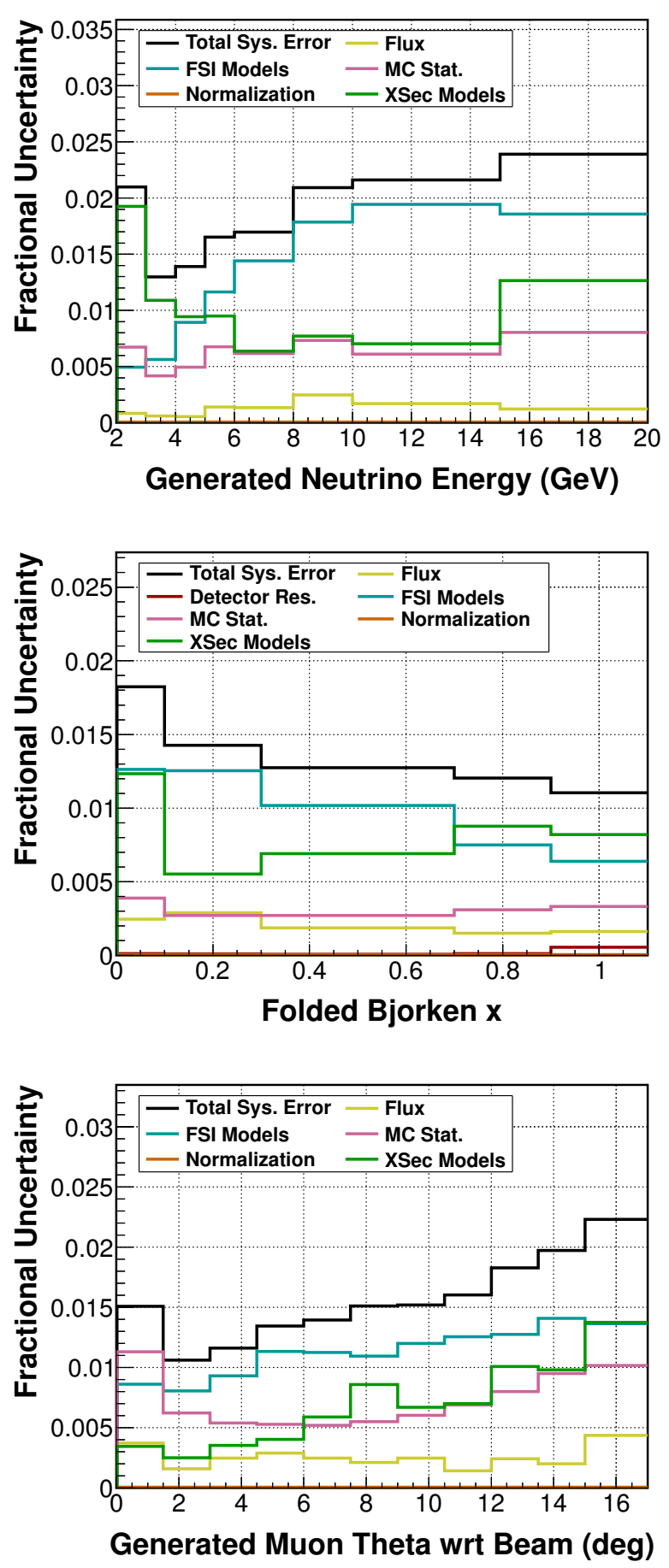

(o) Tracker modules 63-68

Figure A.10: Efficiency and errors on efficiency. For explanation, see top of this section. 


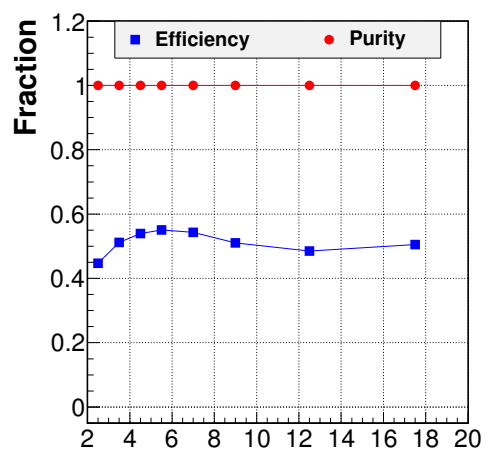

Generated Neutrino Energy (GeV)
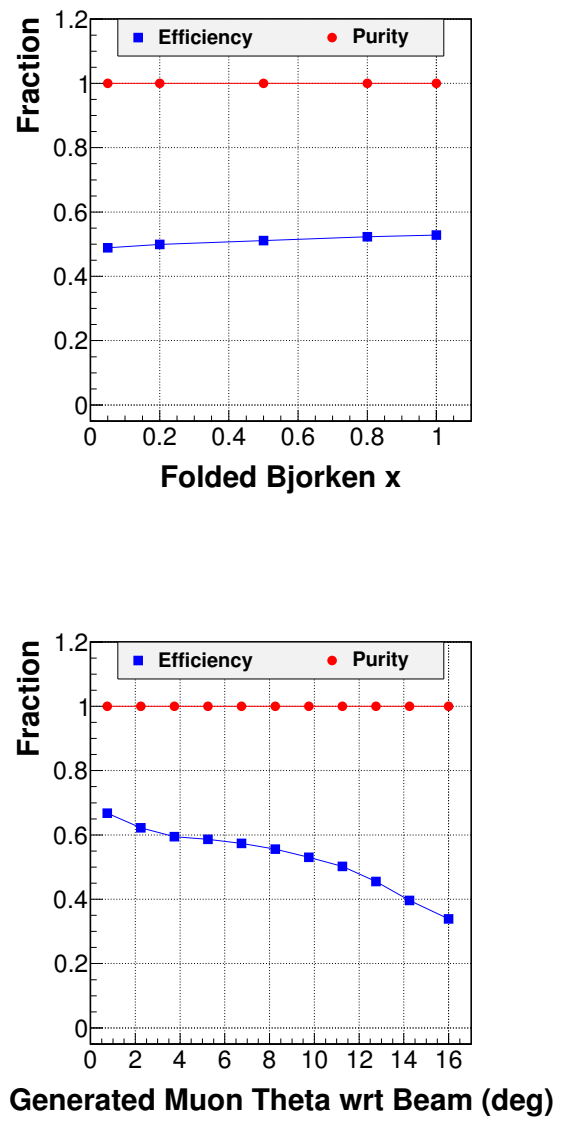
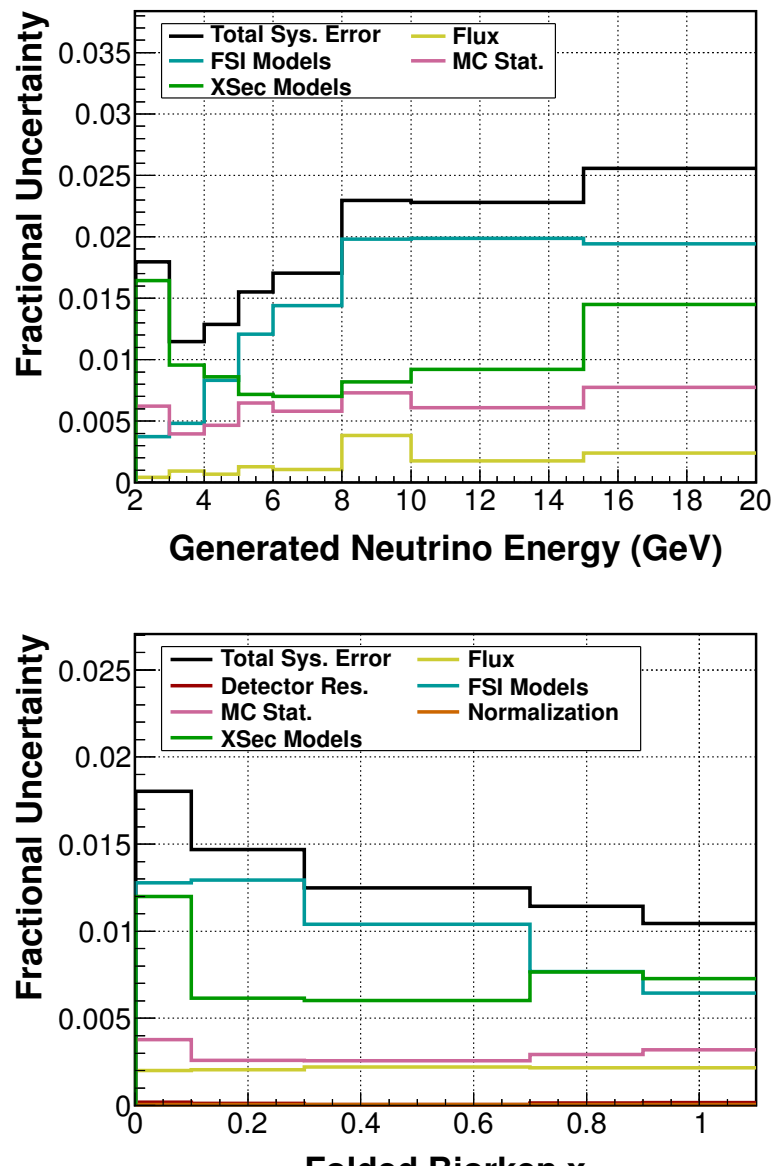

Folded Bjorken x

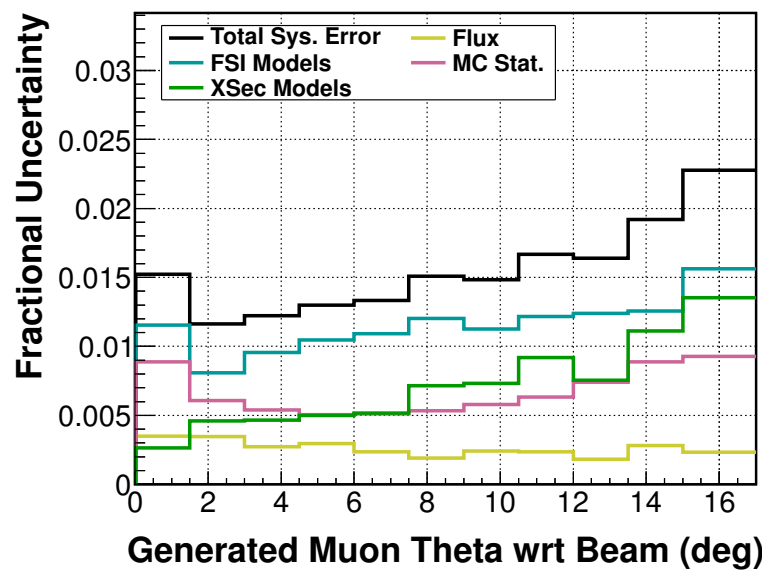

(p) Tracker modules 69-74

Figure A.10: Efficiency and errors on efficiency. For explanation, see top of this section. 


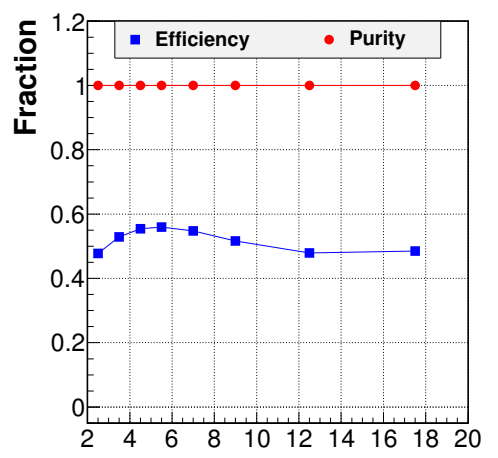

Generated Neutrino Energy (GeV)
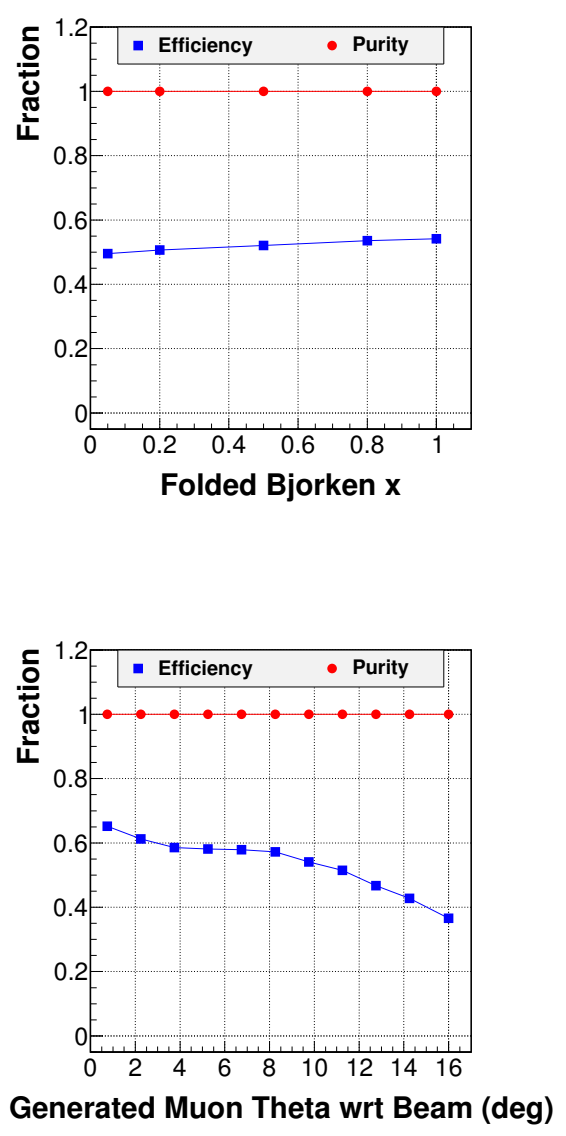
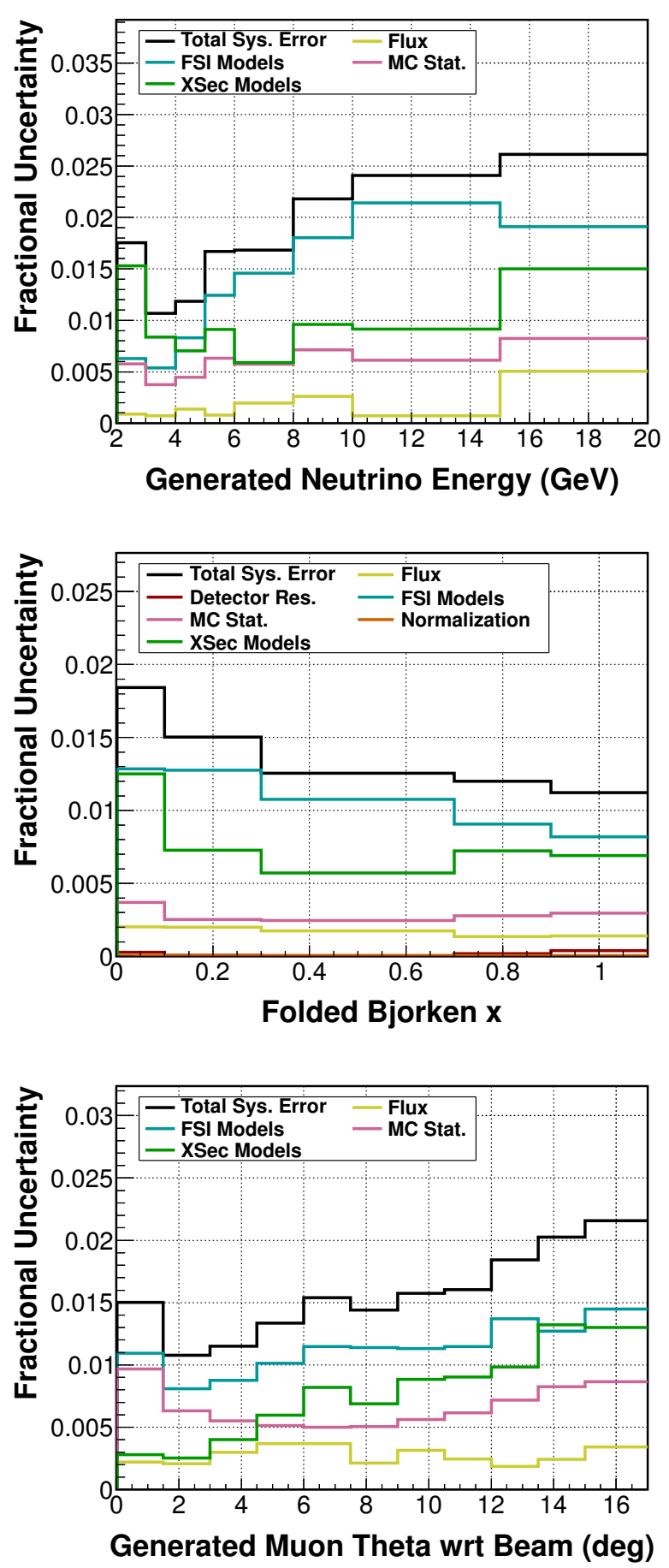

(q) Tracker modules 75-80

Figure A.10: Efficiency and errors on efficiency. For explanation, see top of this section. 


\section{A.7 Migration}

The figures in this section show the simulation's prediction for the number of events migrating due to detector smearing and imperfect reconstruction. The Y-axis is the bin in which the event was generated; the $\mathrm{X}$-axis is the bin in which is was reconstructed.
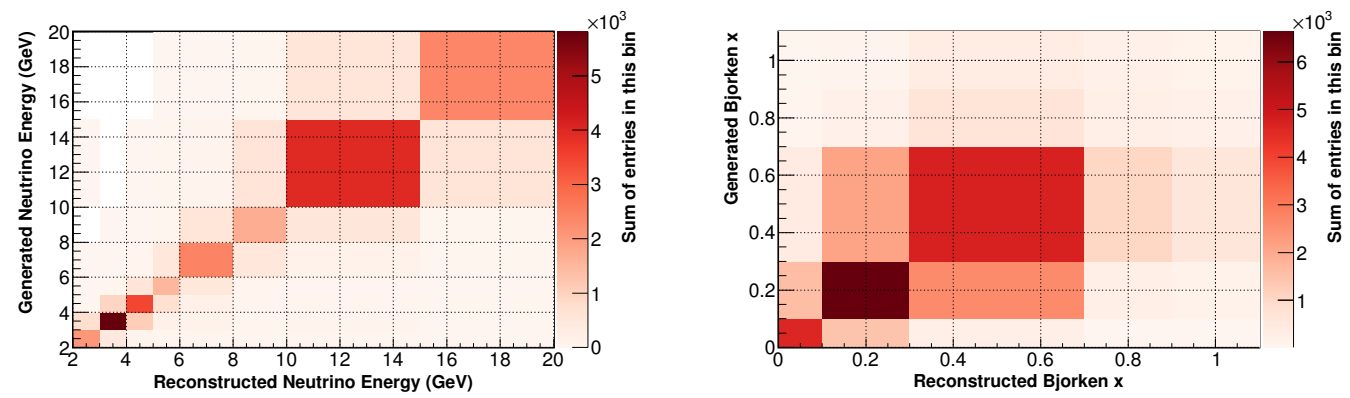

(a) Target 2 iron

Figure A.11: Bin migration. For explanation, see top of this section.
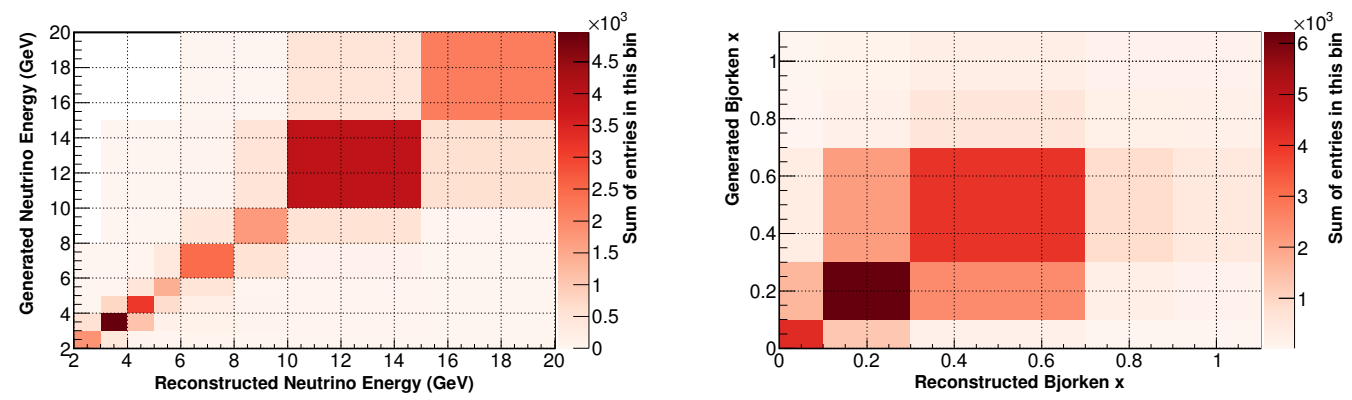

(b) Target 2 lead

Figure A.11: Bin migration. For explanation, see top of this section. 

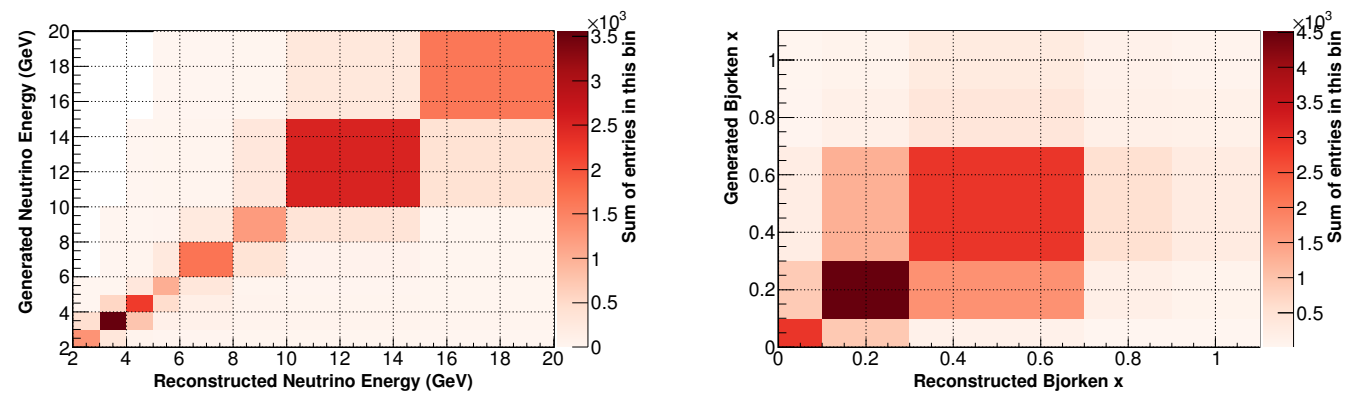

(c) Target 3 carbon

Figure A.11: Bin migration. For explanation, see top of this section.
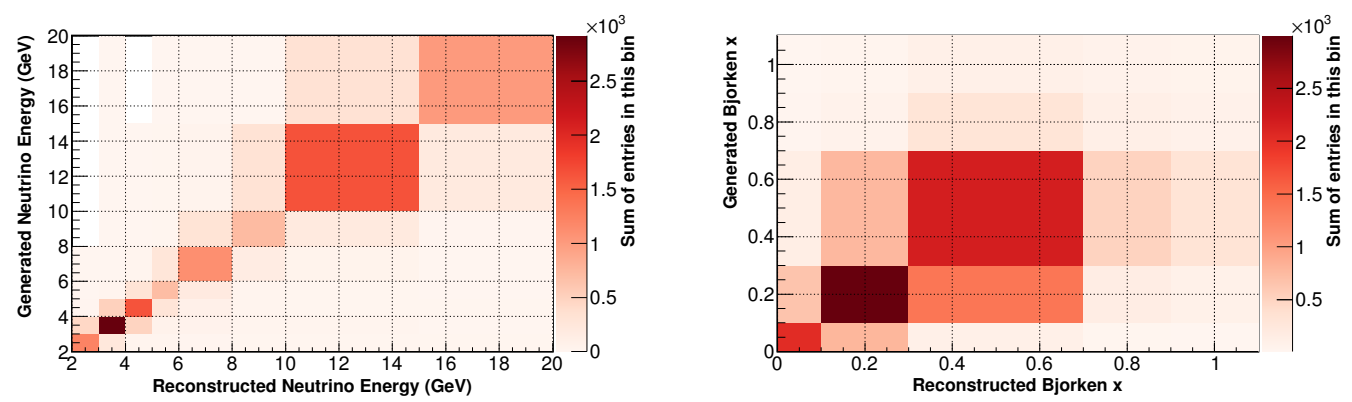

(d) Target 3 iron

Figure A.11: Bin migration. For explanation, see top of this section.
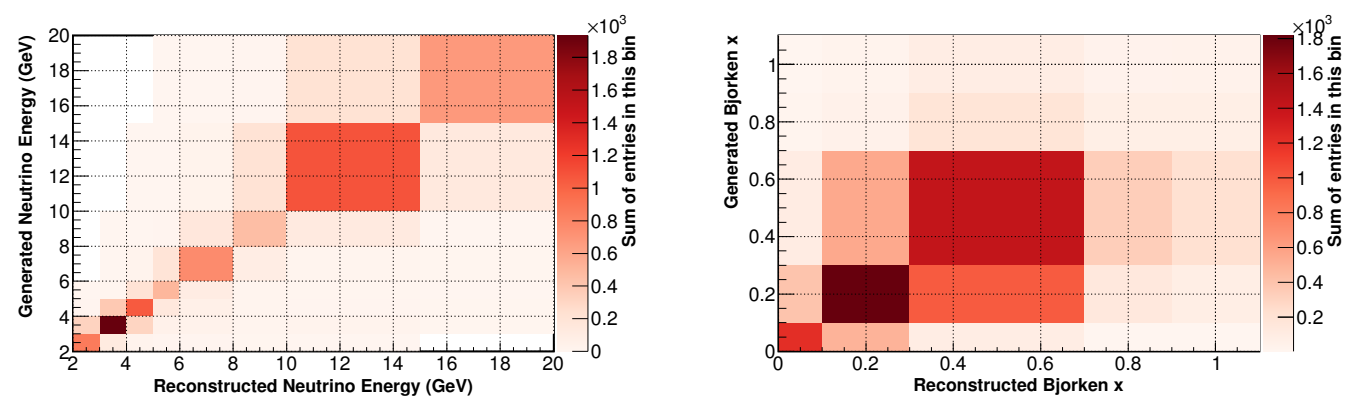

(e) Target 3 lead

Figure A.11: Bin migration. For explanation, see top of this section. 

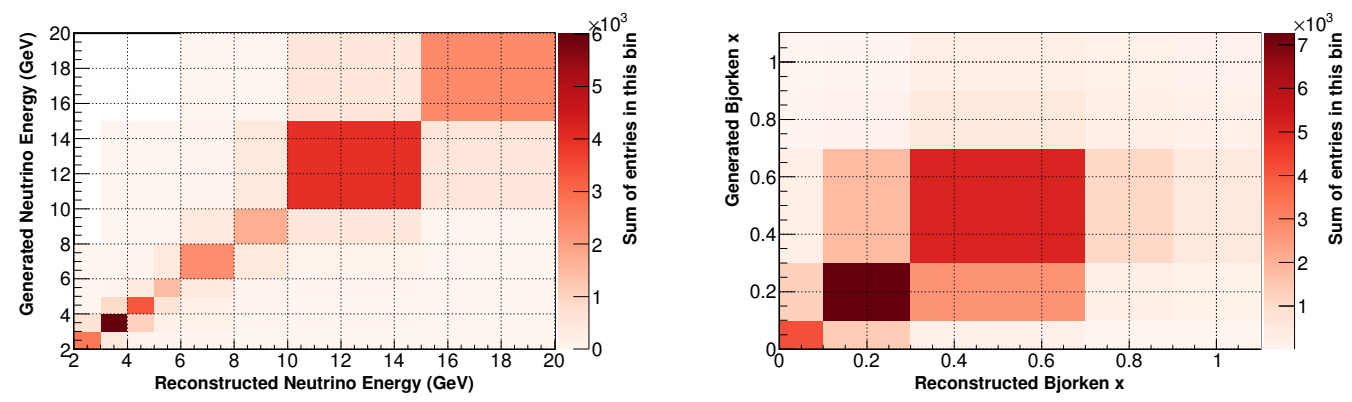

(f) Target 4 lead

Figure A.11: Bin migration. For explanation, see top of this section.
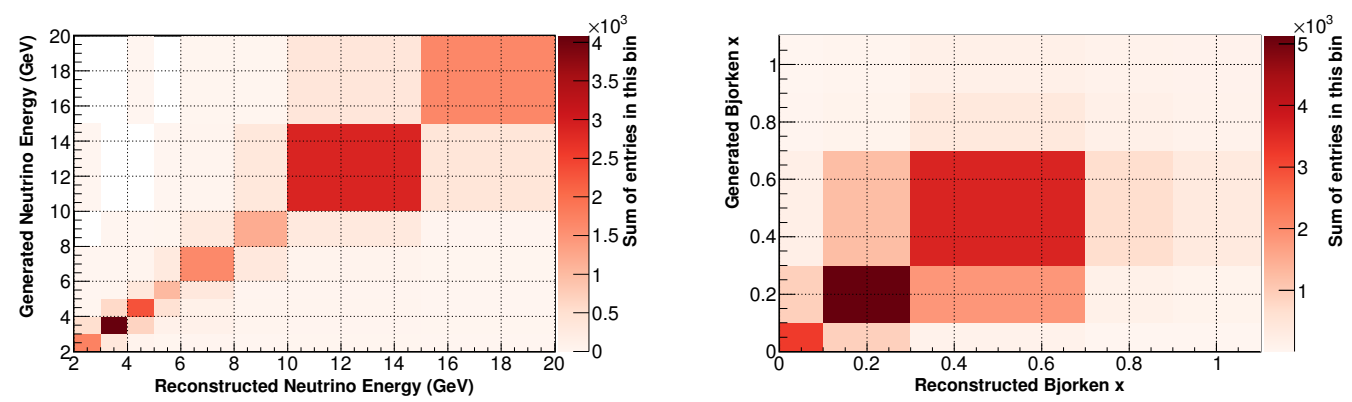

(g) Target 5 iron

Figure A.11: Bin migration. For explanation, see top of this section.
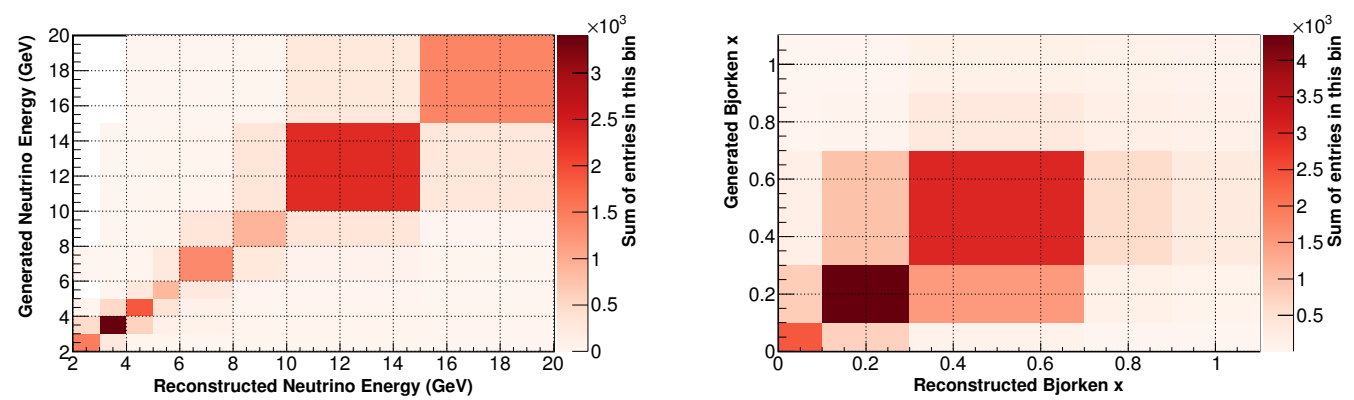

(h) Target 5 lead

Figure A.11: Bin migration. For explanation, see top of this section. 

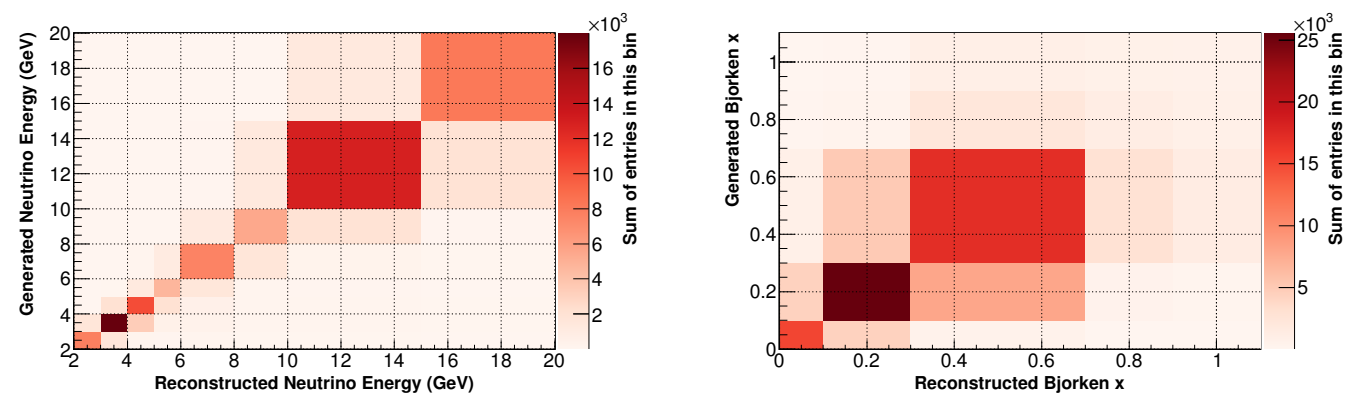

(i) Tracker modules 27-32

Figure A.11: Bin migration. For explanation, see top of this section.
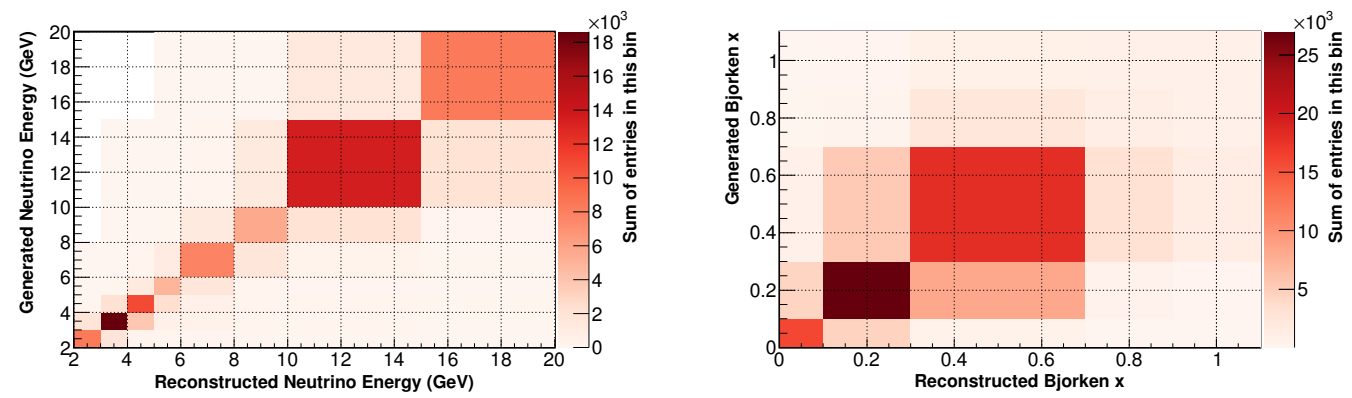

(j) Tracker modules 33-38

Figure A.11: Bin migration. For explanation, see top of this section.
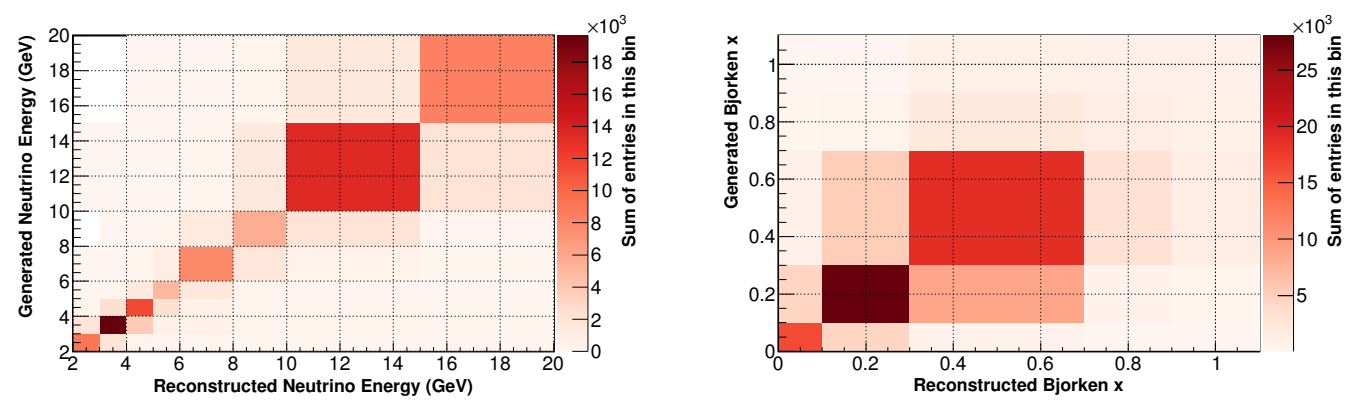

(k) Tracker modules 39-44

Figure A.11: Bin migration. For explanation, see top of this section. 

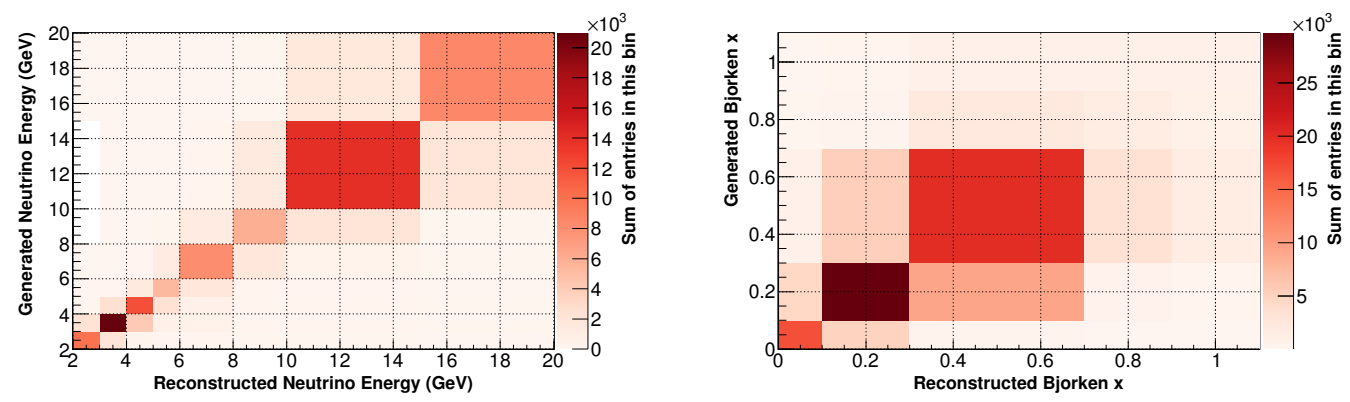

(1) Tracker modules 45-50

Figure A.11: Bin migration. For explanation, see top of this section.
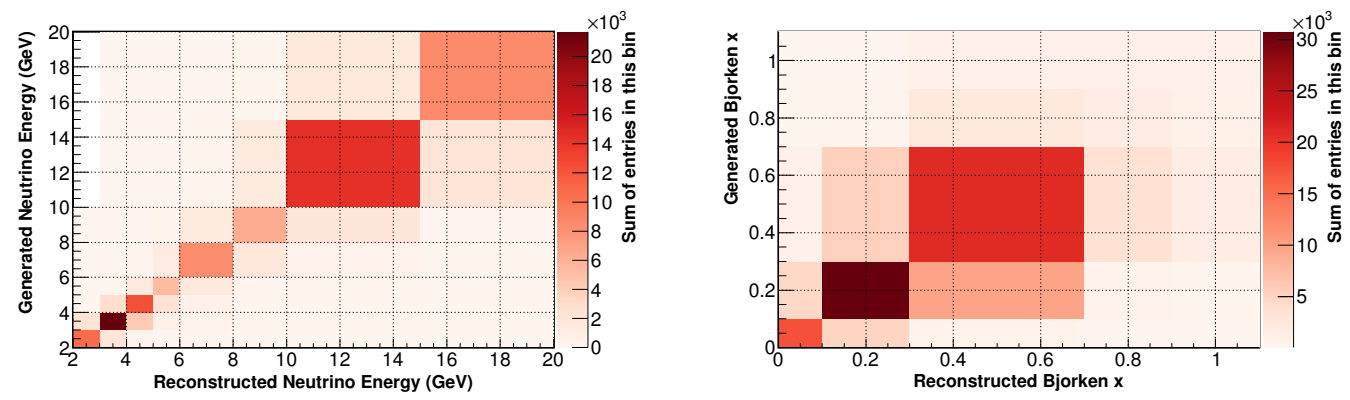

(m) Tracker modules 51-56

Figure A.11: Bin migration. For explanation, see top of this section.
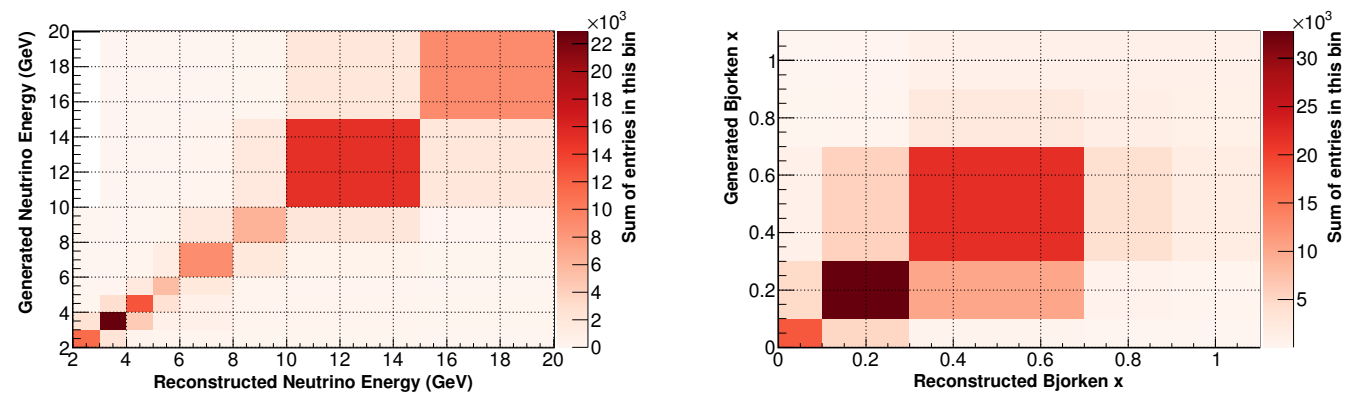

(n) Tracker modules 57-62

Figure A.11: Bin migration. For explanation, see top of this section. 

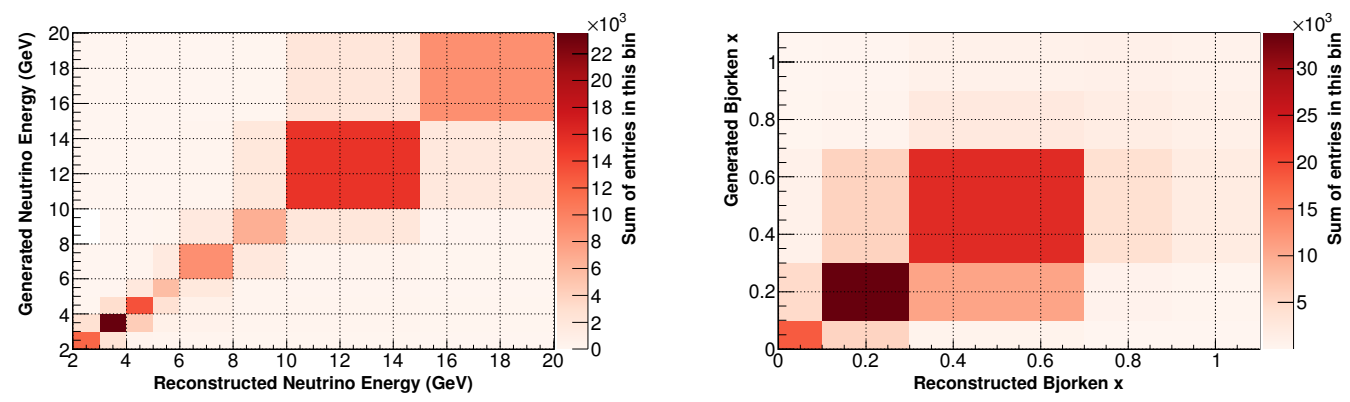

(o) Tracker modules 63-68

Figure A.11: Bin migration. For explanation, see top of this section.
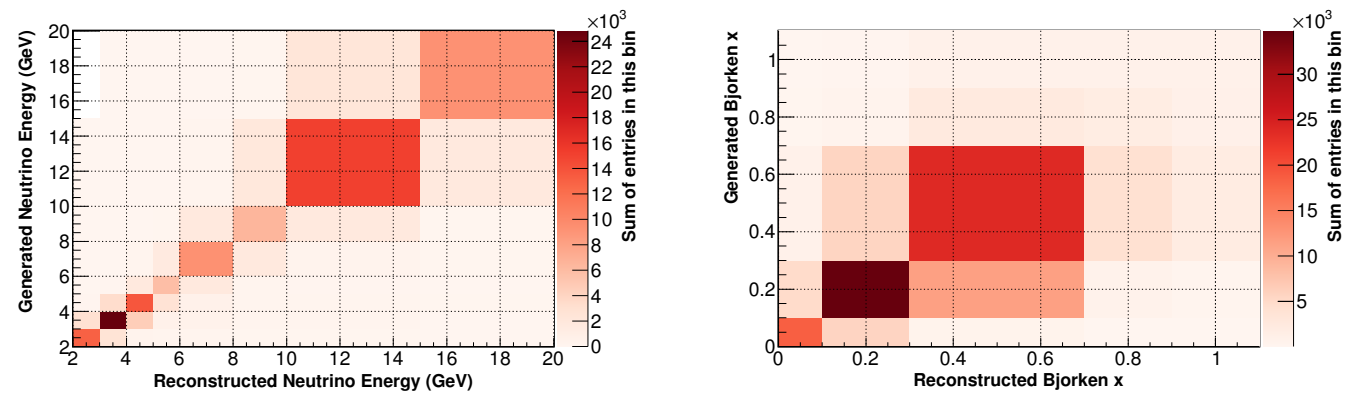

(p) Tracker modules 69-74

Figure A.11: Bin migration. For explanation, see top of this section.
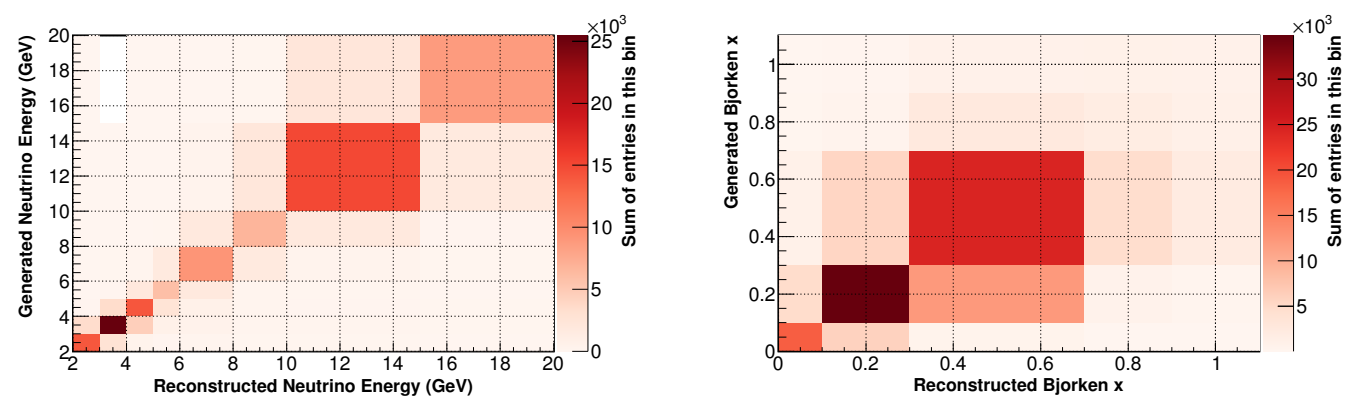

(q) Tracker modules $75-80$

Figure A.11: Bin migration. For explanation, see top of this section. 


\section{A.8 Absolute Cross Sections}

This section contains figures of the measured and predicted total cross section $\sigma$ (left) and differential cross section $\frac{d \sigma}{d x_{b j}}$ (right) for each nucleus. The figures in each row are as follows:

- Top - Measured and absolutely-normalized prediction (MC) of $\sigma$ and $\frac{d \sigma}{d x_{b j}}$. Statistical error is shown on data points and total systematic error is shown in the shaded simulation band.

- Middle - Ratio of measured to predicted cross section. Points show statistical error, shaded band centered on 1 represents full systematic error, and the $\chi^{2}$ calculation accounts for bin-to-bin correlations.

- Bottom - Fractional errors on the absolute cross section. The total error is all sources summed in quadrature.

It is immediate apparent that the overwhelming error is from the flux prediction. The other striking feature is the energy-dependent discrepancy between data and simulation in $\sigma$ (topand middle-left). The primary source of this discrepancy is imperfect simulation of the flux. Poor predictions of the flux do not affect the ratios of cross sections. Each page shows a different material: carbon, iron, lead, scintillator. 

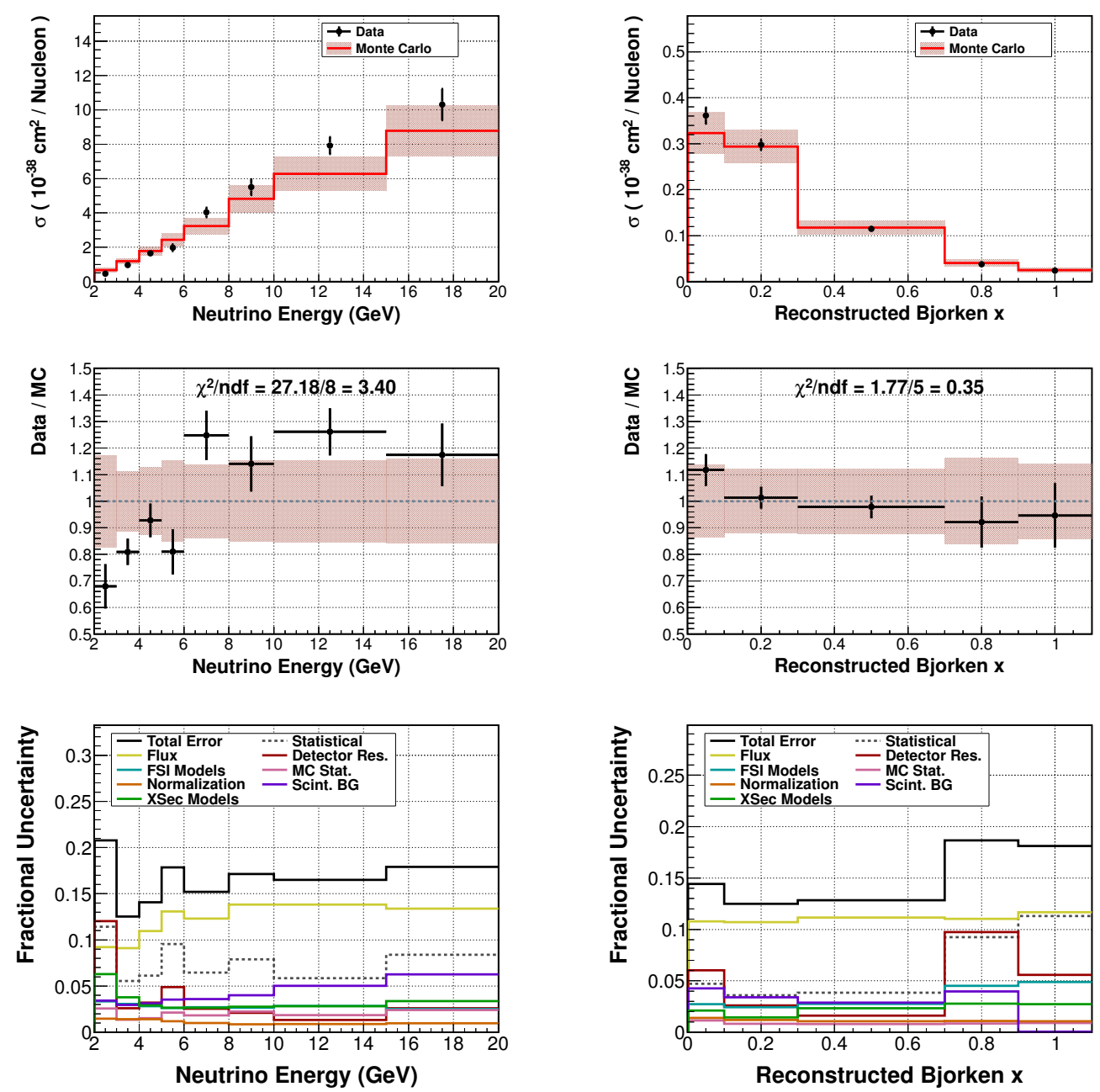

(a) Carbon

Figure A.12: Absolute cross sections. For explanation, see top of this section. 

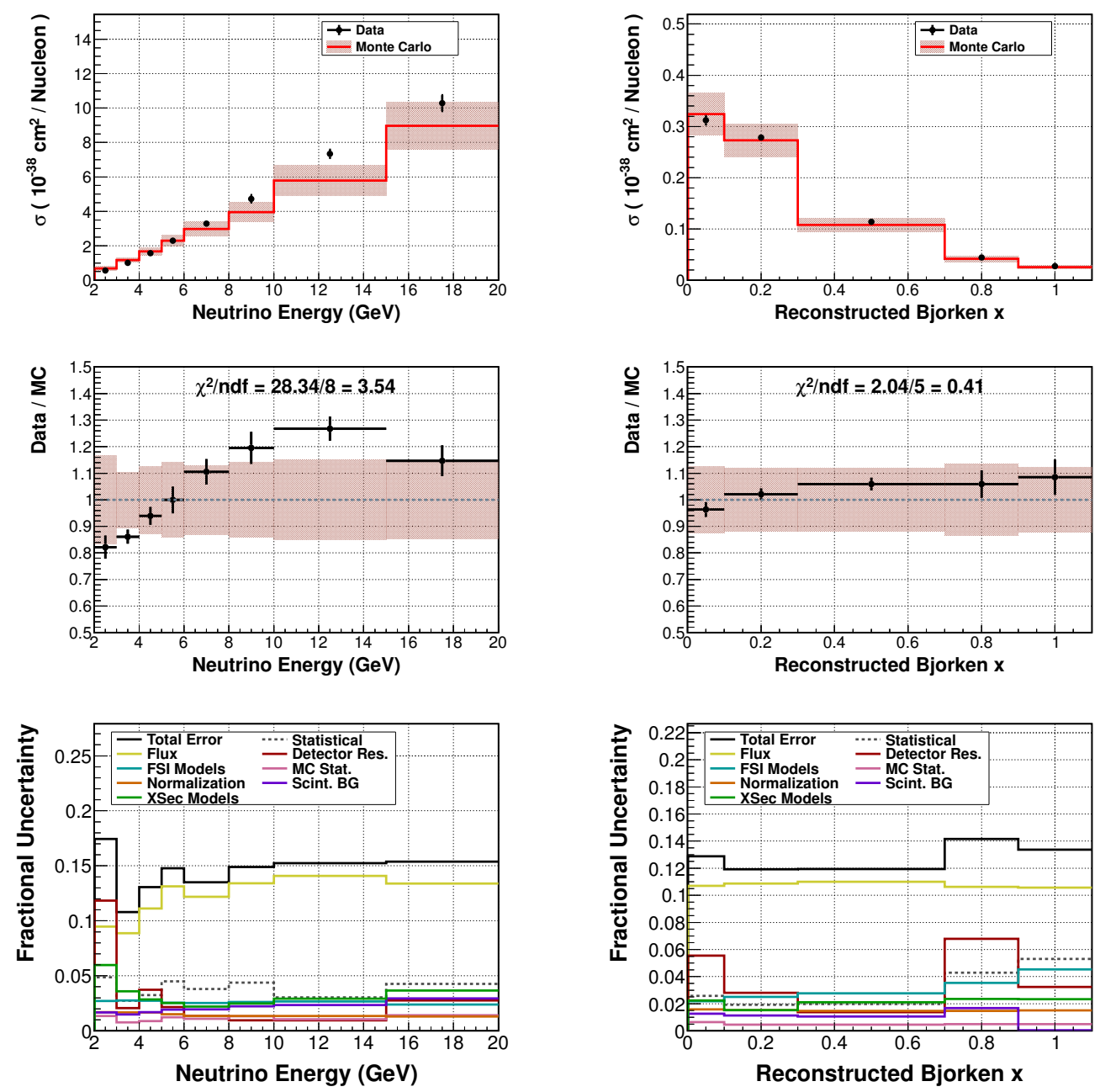

(b) Iron

Figure A.12: Absolute cross sections. For explanation, see top of this section. 

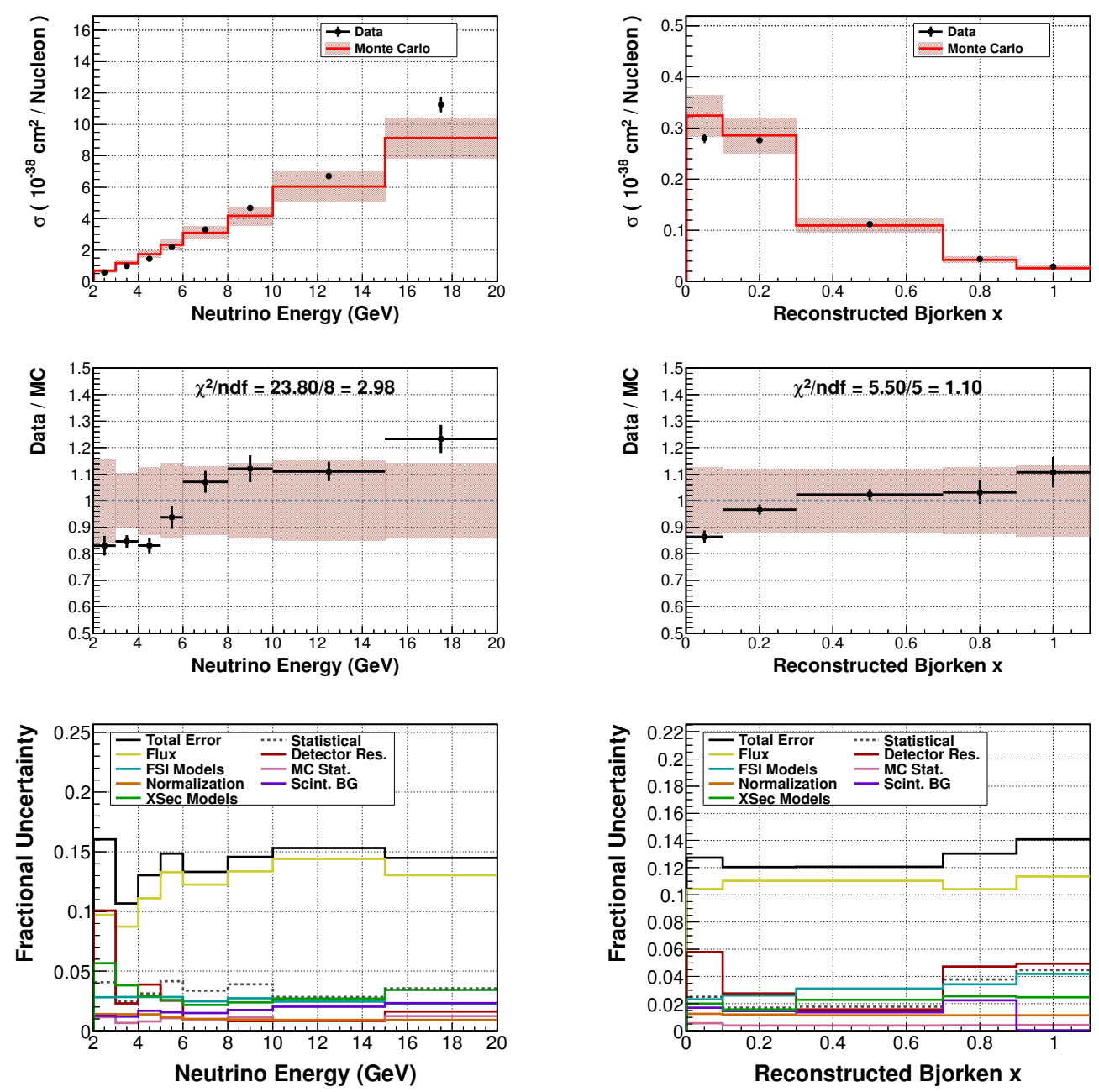

(c) Lead

Figure A.12: Absolute cross sections. For explanation, see top of this section. 

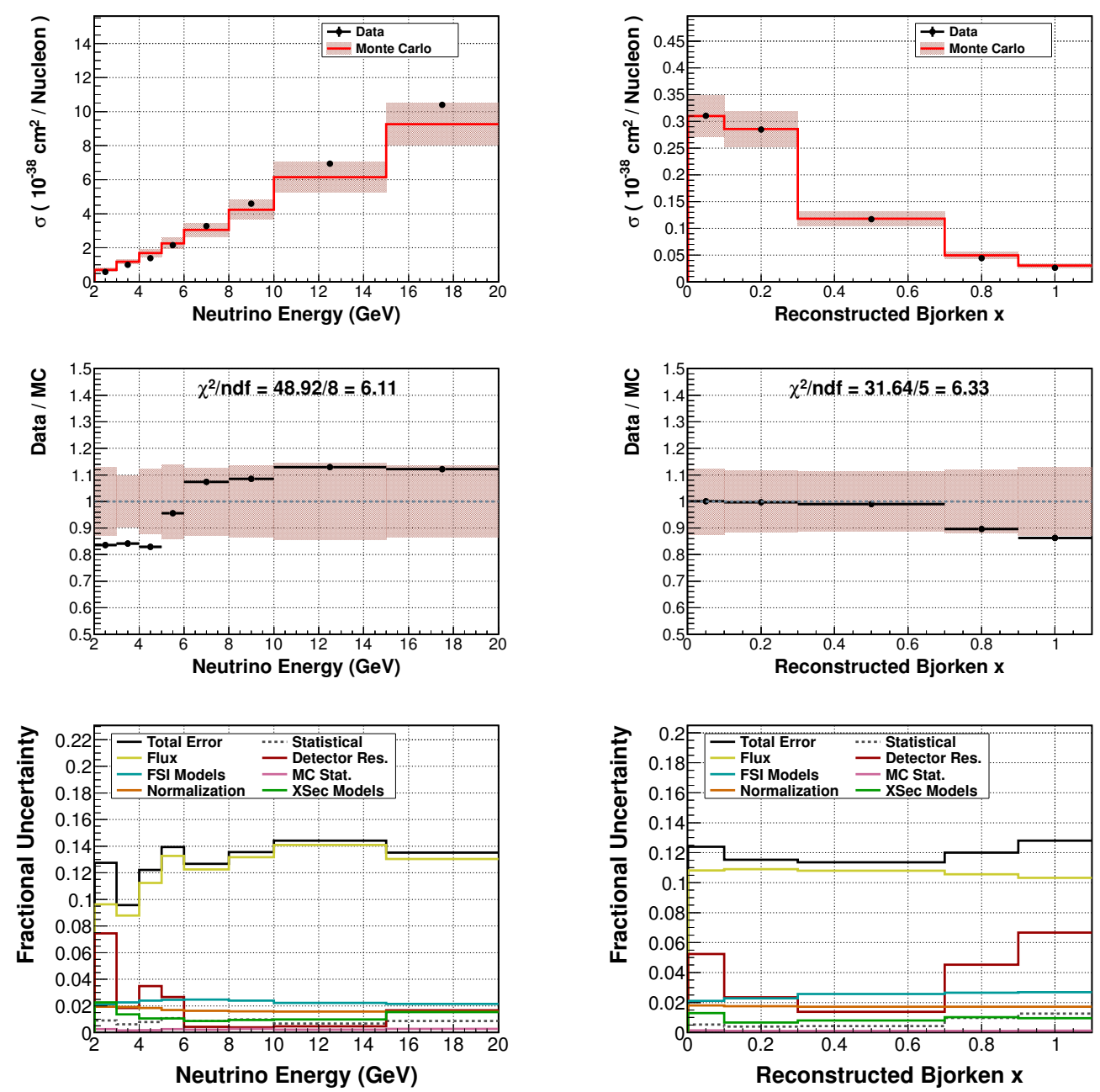

(d) Scintillator

Figure A.12: Absolute cross sections. For explanation, see top of this section. 


\section{A.9 Cross Section Ratios for All Targets}

Figures in this section demonstrate the compatibility of measurements in the different targets. The ratio of total cross section $\sigma$ and differential cross sectio $\frac{d \sigma}{d x_{b j}}$ are shown for $E_{\nu}$ in Sec. A.9.1 and $x_{b j}$ in Sec. A.9.2, respectively. Each bin holds the measurement and prediction from simulation obtained from a single section of passive material. The cross sections of carbon, iron, and lead target sections are normalized by the cross section on scintillator using the faux target pairings of Table 5.6. The isoscalar correction has been applied. These plots suggest that the measurements of the cross sections using different targets are compatible and can thus be added without systematic bias.

\section{A.9.1 Neutrino Energy}

\section{A.9.2 Bjorken $x$}




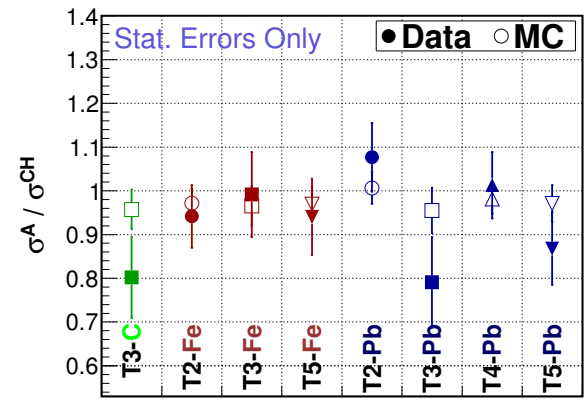

(a) $E_{\nu}=[2,3] \mathrm{GeV}$

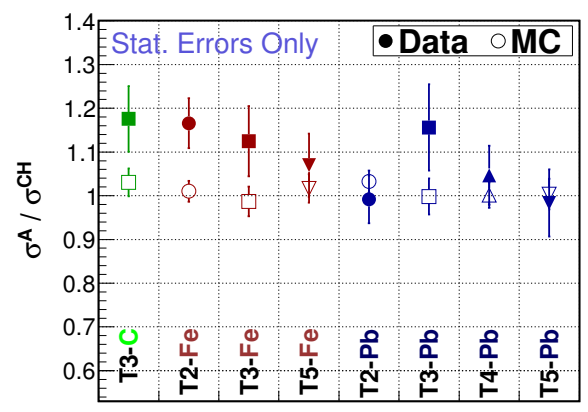

(c) $E_{\nu}=[4,5] \mathrm{GeV}$

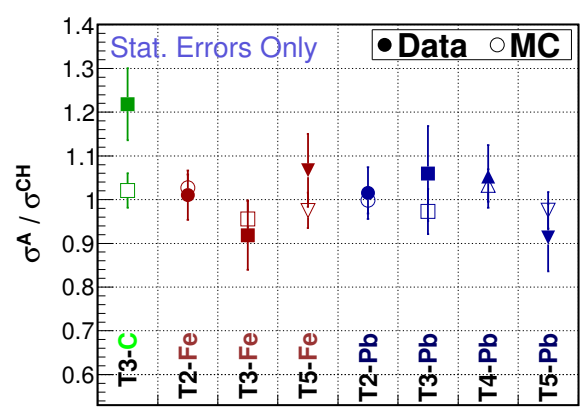

(e) $E_{\nu}=[6,8] \mathrm{GeV}$

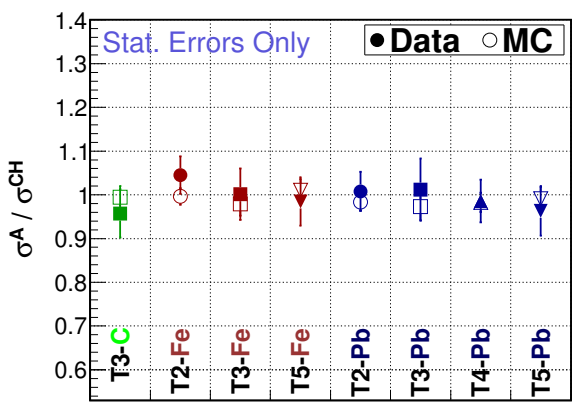

(b) $E_{\nu}=[3,4] \mathrm{GeV}$

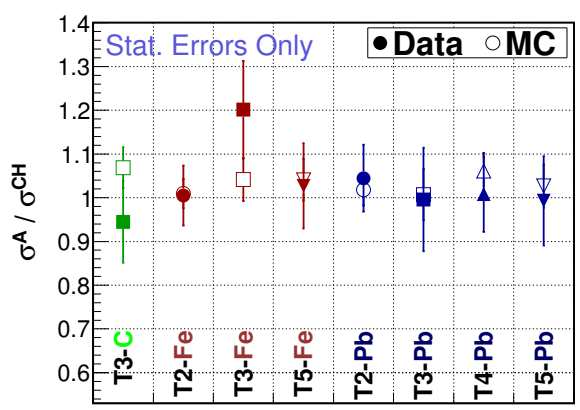

(d) $E_{\nu}=[5,6] \mathrm{GeV}$

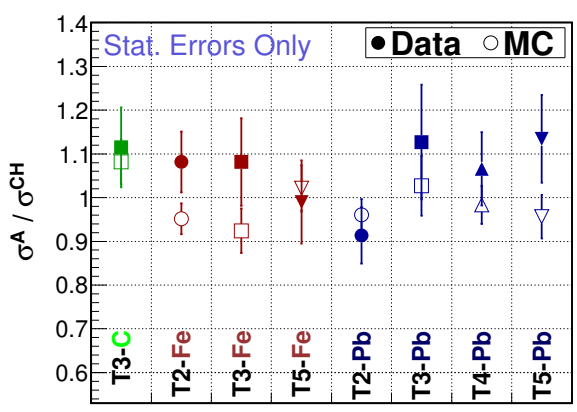

(f) $E_{\nu}=[8,10] \mathrm{GeV}$

Figure A.13: Cross section ratios of carbon, iron, and lead to scintillator measured with each passive target assembly in bins of $E_{\nu}$. Data is shown with solid points, Monte Carlo with hollow points. 


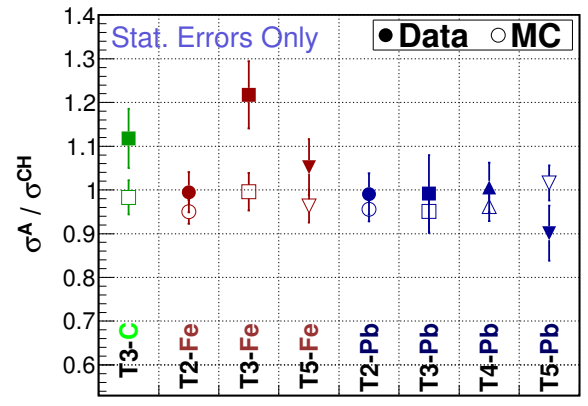

(a) $E_{\nu}=[10,15] \mathrm{GeV}$

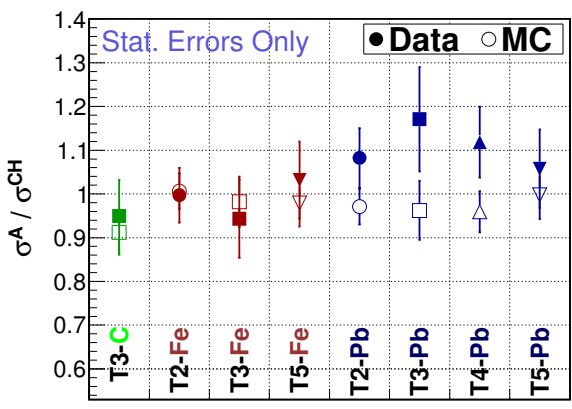

(b) $E_{\nu}=[15,20] \mathrm{GeV}$

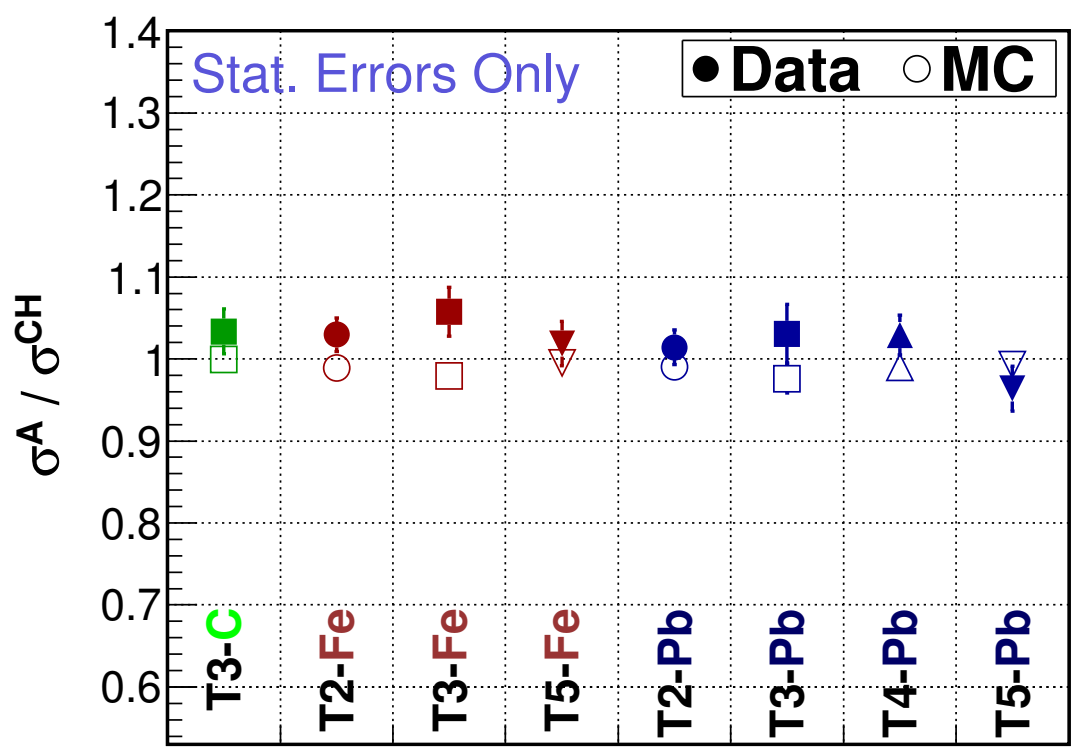

(c) $E_{\nu}=[3,20] \mathrm{GeV}$

Figure A.14: Cross section ratios of carbon, iron, and lead to scintillator measured with each passive target assembly in bins of $E_{\nu}$. Data is shown with solild points, Monte Carlo with hollow points. 


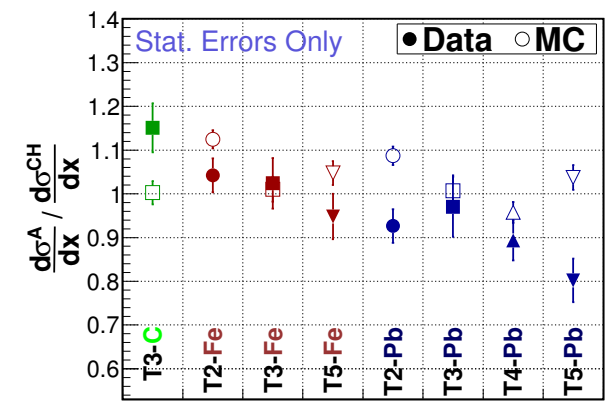

(a) $x_{b j}=[0.0,0.1]$

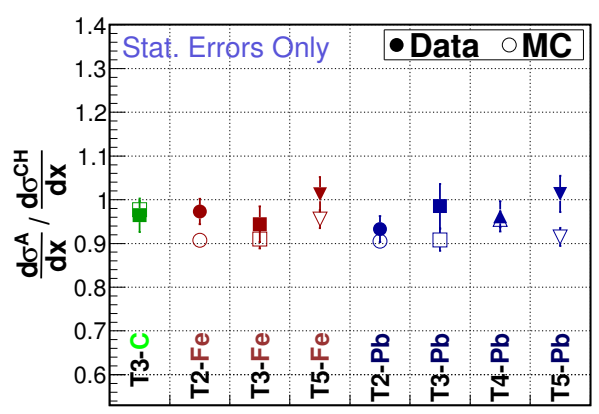

(c) $x_{b j}=[0.3,0.7]$

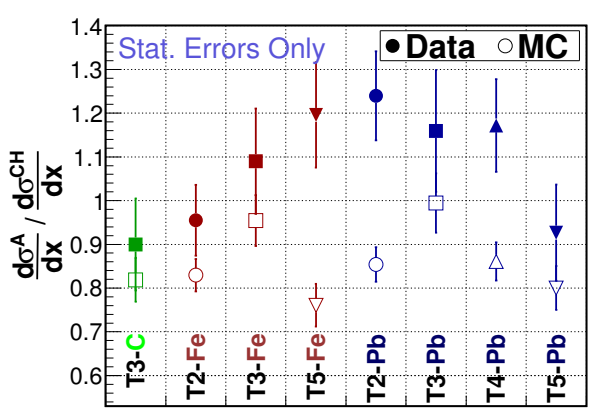

(e) $x_{b j}=[0.9,1.1]$

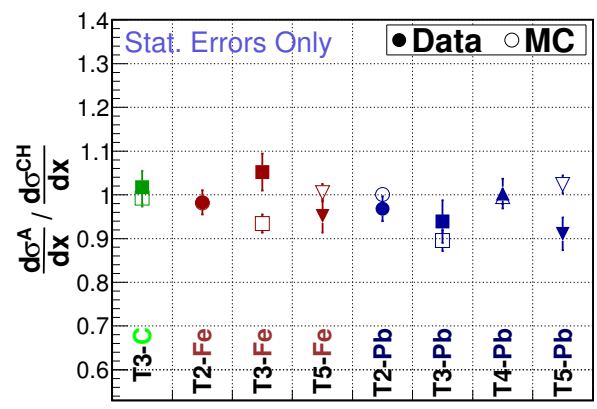

(b) $x_{b j}=[0.1,0.3]$

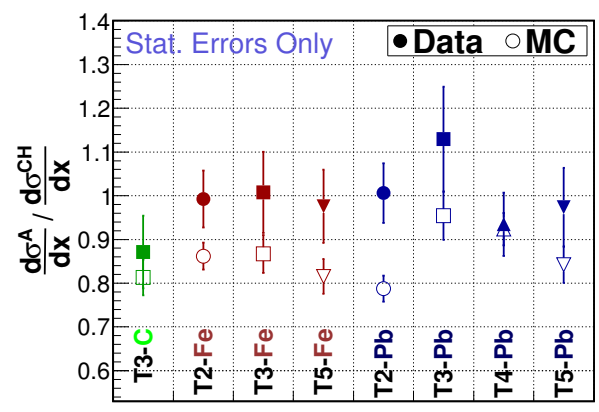

(d) $x_{b j}=[0.7,0.9]$

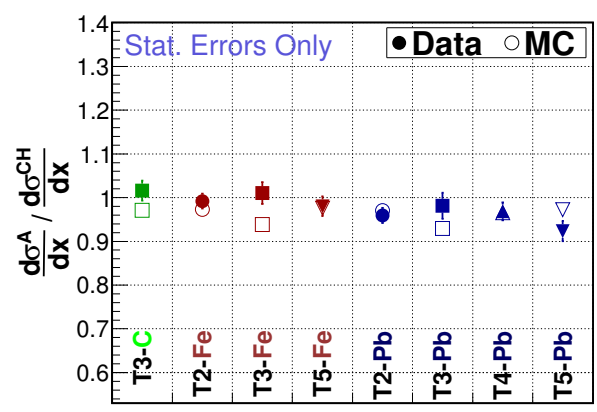

(f) $x_{b j}=[0.0,1.1]$

Figure A.15: Cross section ratios of carbon, iron, and lead to scintillator measured with each passive target assembly in bins of $x_{b j}$. Data is shown with solid points, Monte Carlo with hollow points. 


\section{Appendix B}

\section{Nuclear Target Geometry Calculations}

\section{B.1 Fraction Along Strip Adjacent to Passive Target Occupied by $\mathrm{C} / \mathrm{Fe} / \mathrm{Pb}$}

We want to know the fractional composition of a nuclear target as a function of view coordinates so that we can properly account for the mass of $\mathrm{C}, \mathrm{Fe}$, and $\mathrm{Pb}$ when correcting for visible energy for nearby passive material. To do this we must know the length traversed by the strip through areas occupied by $\mathrm{C}, \mathrm{Fe}$, or $\mathrm{Pb}$. This is done by finding the points at which the strip coordinate intersects borders between the materials. Define the average areal density of the portion of passive material X contributing to the strip's passive energy correction to be

$$
\rho_{\text {avg }}=\frac{\sum_{i} l_{i} \rho_{i}}{\sum_{i} l_{i}}
$$

where $\rho$ is the areal density of a material, $l_{i}$ is the length along the strip that intersects material, and $i$ sums over $\mathrm{C}, \mathrm{Fe}, \mathrm{Pb}$.

These calculations are done in strip units. There are 127 strips in a plane which is $2.14 \mathrm{~m}$ wide, so we define a strip as

$$
1 \text { strip }=\frac{2.14 \mathrm{~m}}{127}=16.85 \mathrm{~mm}
$$

\section{B.1.1 Inner Detector Border}

The ID is a regular hexagon with apothem $a$ of $1.07 \mathrm{~m}$. The apothem is also the width of the hexagon $(w=a)$, the distance from the center of the hexagon to the midpoint of a side. The distance from the center of the hexagon to an intersection of sides is the height of the hexagon 
$h=\frac{2}{\sqrt{3}} w$. A strip in the X-view with strip number $s$ intersects the top (bottom) edge of the ID hexagon at

$$
Y_{\text {top }}(s)=h\left(1-\frac{\left|s-1-\frac{126}{2}\right|}{126}\right)=-Y_{\text {bottom }}(s)
$$

Equation B.3 is valid for all views.

\section{B.1.2 Nuclear Target Materials Border}

Passive nuclear targets 1,2,3, and 5 are segmented in the transverse direction into regions of carbon, iron, and lead. We need to represent the lines defining the border between segments in the coordinate system of each view. In the following subsections we derive the equations for these borders in the $\mathrm{X}$ view. We then rotate in $120(-120){ }^{\circ}$ clockwise about the $\mathrm{Z}$ axis to get the corresponding equations for the $\mathrm{V}(\mathrm{U})$ view

$$
\begin{gathered}
y_{x}=m_{x} * x+b_{x} \\
y_{u}=\frac{u *\left(m_{x} \cos \left(\frac{-2 \pi}{3}\right)+\sin \left(\frac{-2 \pi}{3}\right)\right)-b_{x}}{m_{x} \sin \left(\frac{-2 \pi}{3}\right)-\cos \left(\frac{-2 \pi}{3}\right)} \\
y_{v}=\frac{v *\left(m_{x} \cos \left(\frac{2 \pi}{3}\right)+\sin \left(\frac{2 \pi}{3}\right)\right)-b_{x}}{m_{x} \sin \left(\frac{2 \pi}{3}\right)-\cos \left(\frac{2 \pi}{3}\right)}
\end{gathered}
$$

\section{Target 1/5 Border}

Targets 1 and 5 have the same type of segmentation of iron and lead. The border between lead and iron is $205 \mathrm{~mm}$ in the direction perpendicular to the border line $\alpha$ defined as

$$
\alpha=-x \cos \left(\frac{\pi}{6}\right)+y \sin \left(\frac{\pi}{6}\right) .
$$

Iron is the portion of the hexagon with $\alpha<205 \mathrm{~mm}$. Note that the slope is the same as the slope of rotation for the $\mathrm{U}$ view.

Finding the line perpendicular to B.5 that passes through the same y-intercept yields the equation that describes the border line

$$
y_{x}=x * A_{\alpha}+C_{\alpha}
$$



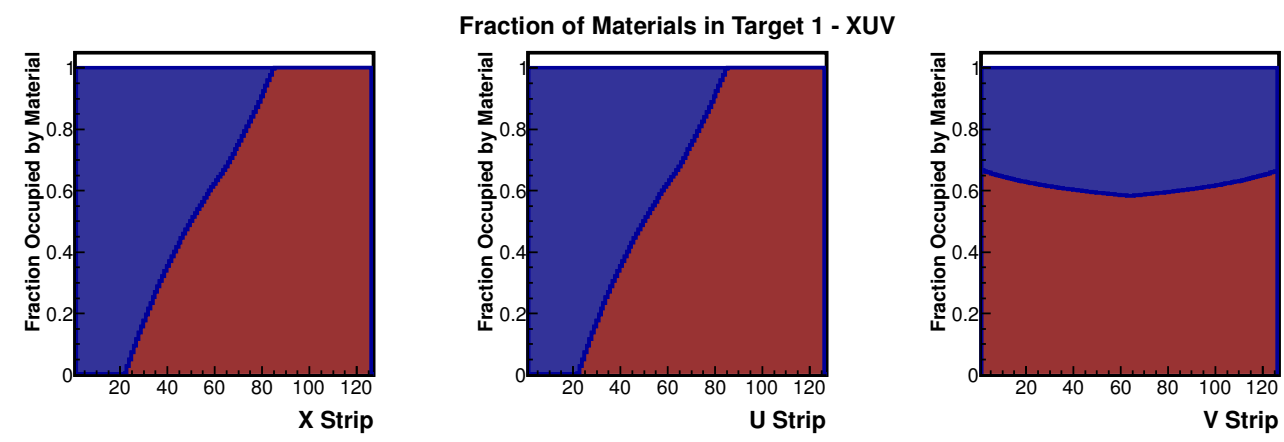

Figure B.1: Fraction of passive target 1 or 5's length contributed by iron (red) and lead (blue) at a strip.

$$
\begin{gathered}
y_{u}=\frac{u *\left(A_{\alpha} \cos \left(\frac{-2 \pi}{3}\right)+\sin \left(\frac{-2 \pi}{3}\right)\right)-C_{\alpha}}{A_{\alpha} \sin \left(\frac{-2 \pi}{3}\right)-\cos \left(\frac{-2 \pi}{3}\right)} \\
y_{v}=\frac{v *\left(A_{\alpha} \cos \left(\frac{2 \pi}{3}\right)+\sin \left(\frac{2 \pi}{3}\right)\right)-C_{\alpha}}{A_{\alpha} \sin \left(\frac{2 \pi}{3}\right)-\cos \left(\frac{2 \pi}{3}\right)}
\end{gathered}
$$

where $A_{\alpha}=-\tan \left(\frac{\pi}{6}\right)$ and $C_{\alpha}=\frac{12.166}{\sin \left(\frac{\pi}{6}\right)}$. Lead is above this line in the $\mathrm{X}$ and $\mathrm{U}$ view; iron is above the line in V. The fraction of target 1 or 5's length made of each material is shown in Fig. B.1.

\section{Target 2 Border}

The border between lead and iron is $205 \mathrm{~mm}$ in the direction perpendicular to the border line $\beta$ defined as

$$
\beta=x \cos \left(\frac{\pi}{6}\right)+y \sin \left(\frac{\pi}{6}\right)
$$

Iron is the portion of the hexagon with $\beta<205 \mathrm{~mm}$. Note that the slope is the same as the slope of rotation for the $\mathrm{V}$ view.

Finding the line perpendicular to B.5 that passes through the same y-intercept yields the equation that describes the border line

$$
\begin{gathered}
y_{x}=x * A_{\beta}+C_{\beta} \\
y_{u}=\frac{u *\left(A_{\beta} \cos \left(\frac{-2 \pi}{3}\right)+\sin \left(\frac{-2 \pi}{3}\right)\right)-C_{\beta}}{A_{\beta} \sin \left(\frac{-2 \pi}{3}\right)-\cos \left(\frac{-2 \pi}{3}\right)}
\end{gathered}
$$



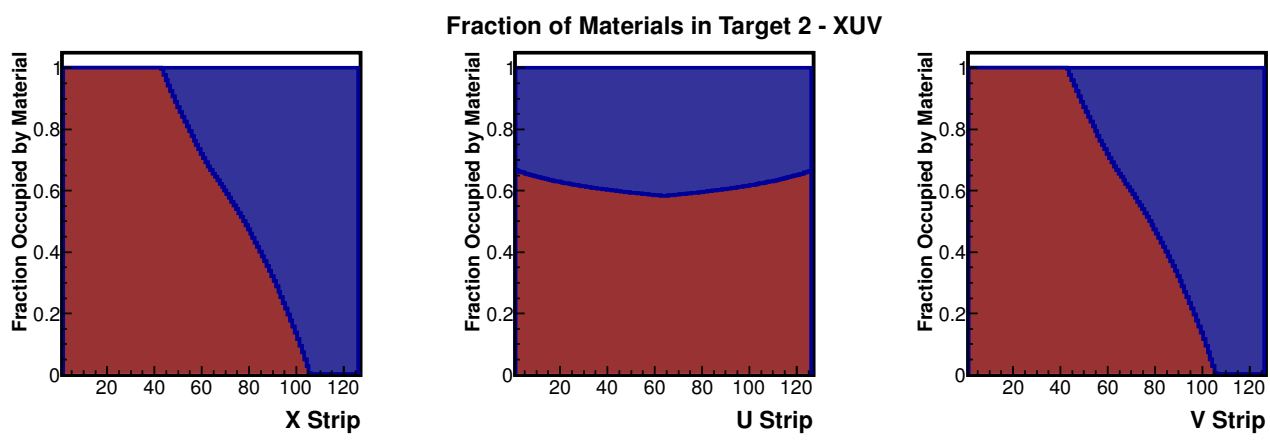

Figure B.2: Fraction of passive target 2's length contributed by iron (red) and lead (blue) at a strip.

$$
y_{v}=\frac{v *\left(A_{\beta} \cos \left(\frac{2 \pi}{3}\right)+\sin \left(\frac{2 \pi}{3}\right)\right)-C_{\beta}}{A_{\beta} \sin \left(\frac{2 \pi}{3}\right)-\cos \left(\frac{2 \pi}{3}\right)}
$$

where $A_{\beta}=\tan \left(\frac{\pi}{6}\right)$ and $C_{\beta}=\frac{12.166}{\sin \left(\frac{\pi}{6}\right)}$. Lead is above this line in the $\mathrm{X}$ and $\mathrm{V}$ view; iron is above the line in U. The fraction of target 2's length made of each material is shown in Fig. B.2.

\section{Target 3 Borders}

Target 3 has carbon, iron, and lead. Carbon makes up 1/2 of the hexagon, iron 1/3, and lead 1/6. The carbon half is found on the half with $\beta>0$, where $\beta$ is defined in Eq. B.7. The border between carbon and iron-lead at $\gamma=0$ where with $\gamma$ defined as

$$
\gamma=-x \sin \left(\frac{\pi}{6}\right)+y \cos \left(\frac{\pi}{6}\right)
$$

Carbon is in the portion of the hexagon with $\gamma>0$. The iron-lead half at $\gamma<0$ is divided at $x=0$ so that lead is at $x>0$ and iron at $x<0$.

Finding the line perpendicular to B.9 that passes through the same y-intercept yields the equation that describes the border line between carbon and iron-lead

$$
\begin{gathered}
y_{x}=x * A_{\beta} \\
y_{u}=0
\end{gathered}
$$



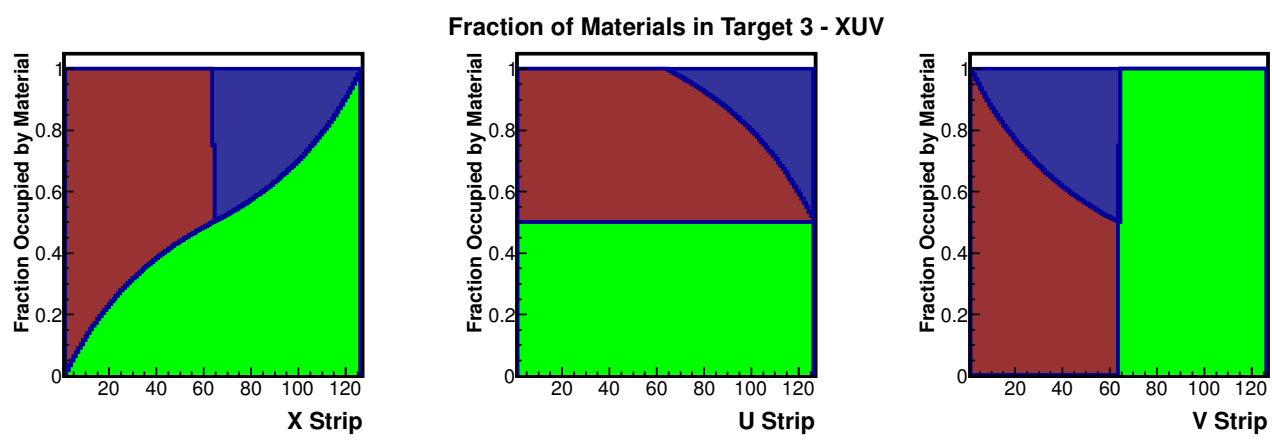

Figure B.3: Fraction of passive target 3's length contributed by carbon (green), iron (red), and lead (blue) at a strip.

$$
y_{v}=\frac{v *\left(A_{\beta} \cos \left(\frac{2 \pi}{3}\right)+\sin \left(\frac{2 \pi}{3}\right)\right)}{A_{\beta} \sin \left(\frac{2 \pi}{3}\right)-\cos \left(\frac{2 \pi}{3}\right)}
$$

where $A_{\beta}=\tan ^{-1}\left(\frac{\pi}{3}\right)$. Carbon is above this line.

The division between iron and lead is given by

$$
\begin{gathered}
x_{x}=0 \\
y_{u}=-u * \tan ^{-1}\left(\frac{-2 \pi}{3}\right) \\
y_{v}=-v * \tan ^{-1}\left(\frac{2 \pi}{3}\right)
\end{gathered}
$$

When the center strip in the $\mathrm{X}$ view is hit, the $\mathrm{Fe} / \mathrm{Pb}$ are split evenly. Fe lies below the line of $y_{u}$ and above the line of $y_{v}$. The fraction of target 3's length made of each material is shown in Fig. B.3

\section{B.2 Fiducial Area of Nuclear Targets}

In this section we describe how to calculate the fiducial area of passive target sections. The fiducial area is defined as the area being within a regular hexagon of apothem $a$ (usually 850 $\mathrm{mm}$ ) centered on the detector's longitudinal axis that is also at least a distance $d$ (usually 25 $\mathrm{mm}$ ) away from the border between materials in the target. The area of a hexagon and the distance from the center to a point will be used repeatedly and are shown in Eq. B.12 


$$
\begin{gathered}
\text { hexArea }=\frac{6 * a^{2}}{\sqrt{3}} \\
\text { centerToPoint } \frac{2 a}{\sqrt{3}}
\end{gathered}
$$

\section{B.2.1 Targets 1, 2, 5}

Targets 1, 2, and 5 are composed of the same fractions of iron and lead. Targets 1 and 5 have the same orientation, and target 2 has the same orientation after a $180{ }^{\circ}$ rotation about the y-axis. The division between iron and lead is offset from the center of the hexagon by $205 \mathrm{~mm}$ in the direction defined by B.5 for targets 1 and 5, and B.7 for target 2. The division is placed so iron occupies more space. The following calculations are only valid if the apothem is more than twice as long as the distance to the division (i.e. for $a>410 \mathrm{~mm}$ ).

\section{Iron}

The area occupied by iron is equal to half of the area of a regular hexagon, plus the area added by the $205 \mathrm{~mm}$ offset, less the buffer area removed around the diviion.

$$
\begin{array}{r}
\text { offsetArea }=2 * a *(205 \mathrm{~mm}) \\
\text { bufferArea }=2 a d \\
A_{\mathrm{Fe}}^{125}=\frac{\text { hexArea }}{2}+\text { offsetArea }- \text { bufferArea } \\
A_{\mathrm{Fe}}^{125}=\frac{\text { hexArea }}{2}+2 * a *(205 \mathrm{~mm})-2 a d
\end{array}
$$




\section{Lead}

The area occupied by lead is equal to half of the area of a regular hexagon, less the area added by the $205 \mathrm{~mm}$ offset, less the buffer area removed around the diviion.

$$
\begin{array}{r}
\text { offsetArea }=2 * a *(205 \mathrm{~mm}) \\
\text { bufferArea }=2 a d \\
A_{P b}^{125}=\frac{\text { hexArea }}{2}-\text { offsetArea }- \text { bufferArea } \\
A_{P b}^{125}=\frac{\text { hexArea }}{2}-2 * a *(205 \mathrm{~mm})-2 a d
\end{array}
$$

\section{B.2.2 Target 3}

At the most basic description, target 3 comprises a hexagon with a 3 sextant piece of carbon, 2 sextant piece of iron, and 1 sextant piece of lead. However, the requirement that the fiducial area excludes regions a distance $d$ near the border between materials complicates this description. We calculate the area of the pieces by starting with the basic description and subtracting.

\section{Carbon}

The carbon section begins with three sextants. The area subtracted for the buffer region is a trapezoid. We describe the trapezoid as a rectangle with two triangles removed. The rectangle has a width equal to the point-to-point distance of the hexagon and a height $d$. The removed rectangles account for the sloping sides of the hexagon.

$$
\begin{array}{r}
\text { areaOfRectangle }=2 d t \\
\text { areaOfTriangle }=.5 d\left(d \tan \left(\frac{\pi}{6}\right)\right) \\
A_{C}^{3}=\frac{\text { hexArea }}{2}-(\text { areaOfRectangle }-2 * \text { areaOfTriangle }) \\
A_{C}^{3}=\frac{\text { hexArea }}{2}-\left(2 d t-\left(d \tan \left(\frac{\pi}{6}\right)\right)\right)
\end{array}
$$




\section{Iron}

Iron begins with two sextants. The area subtracted for the buffer region is described as two rhombi. One rhombus accounts for the buffer along the border with carbon; it has a width $t$ and height $d$. The other rhombus acounts for the buffer along the border with lead; it has a height $d$ and a width $t-d$, because $d$ of the width has already been acounted for by the carbon rhombus.

$$
\begin{array}{r}
\text { areaOfRhombus } C=d t \\
\text { areaOfRhombusPb }=d(t-d) \\
A_{F e}^{3}=\frac{\text { hexArea }}{3}-\text { areaOfRhombusC }- \text { areaOfRhombusPb } \\
A_{F e}^{3}=\frac{\text { hexArea }}{3}-d t-d(t-d)
\end{array}
$$

\section{Lead}

The lead begins with one sextant. The area subtracted for the buffer region is described as two trapezoids, each with thickness $d$. One trapezoid accounts for the buffer along the border with carbon; it has a major length $t$ and a minor length $t-d$. One trapezoid accounts for the buffer along the border with iron; it has a major length $t-d$ and a minor length $t-d-2 d \tan \left(\frac{\pi}{6}\right)$.

$$
\begin{array}{r}
\text { areaOfTrapC }=.5 t(t-d) \\
\text { areaOfTrapFe }=.5(t-d)\left(t-d-2 d \tan \left(\frac{\pi}{6}\right)\right. \\
A_{P b}^{3}=\frac{\text { hexArea }}{6}-\text { areaOfTrapC }- \text { areaOfTrapFe } \\
A_{P b}^{3}=\frac{\text { hexArea }}{6}-.5 t(t-d)-.5(t-d)\left(t-d-2 d \tan \left(\frac{\pi}{6}\right)\right.
\end{array}
$$




\section{Appendix C}

\section{Kalman Filters}

\section{C.1 Track Fitting}

To fit tracks, MINERvA uses an implementation of the well known Kalman filter method [40], which is a local least-squares estimator. In the application to track fitting, the Kalman filter will minimize the sum of standardized distance between the position of the energy deposited in a layer of scintillator and the estimate of the track's parameters as it passed through that layer of scintillator.

The clusters used to form the track are the measurements which we try to fit to the path of a charged particle under the hypothesis of our track model. For each cluster, we create a track state, which is the estimate of the track's parameters in a plane of scintillator The track

parameters are chosen to be $\vec{q}=\left(x, y, \frac{d x}{d z}, \frac{d y}{d z}, \frac{q}{p}\right)$. The z-position of the state is the same as the z-position of the scintillator plane which contains the cluster. States also carry the covariance matrix which relates the errors on track parameter estimates. The pairing of a cluster and state is called a node. For each node, we can calculate a residual, which is the standardized distance between the cluster and state.

The goal of track fitting is to minimize the $\chi^{2}$, which is the sum of nodes' residuals. This minimization is accomplished trough the following steps

- Seed: Make an initial guess of track parameters at one end of the track

- Predict: Use state $\vec{q}_{k}$ to predict state $\vec{q}_{k+1}$ 
- Filter: Update the predicted state $\vec{q}_{k+1}$ based on the measured position of the cluster in that plane

- Smooth: Use filtered state $\vec{q}_{k+1}$ to back-predict state $\vec{q}_{k}$ and update again the parameters of $\vec{q}_{k+1}$

To accomplish minimization one uses an initial guess of track parameters at one layer of scintillator to predict the track parameters at the next layer of scintillator. This prediction is compared with the measurement. The main components of the routine are described in detail below.

\section{C.1.1 Seed State}

The seed state is created during $3 \mathrm{D}$ track merging out of the two or three $2 \mathrm{D}$ track candidates. The $\mathrm{x}$ and $\mathrm{y}$ positions and slopes of the seed are calculated with an appropriate superposition of the track candidates' starting positions and slopes. The momentum of the seed state is estimated from the track length using a fast parametrization of the Bethe-Bloch formula.

\section{C.1.2 Predict}

A state is used to predict the position of the next state using the simple equations of a linear track model:

$$
\begin{array}{ll}
x_{k+1}=x_{k}+\left(z_{k+1}-z_{k}\right)\left(\frac{d x}{d z}\right)_{k} & \left(\frac{d x}{d z}\right)_{k+1}=\left(\frac{d x}{d z}\right)_{k} \\
y_{k+1}=y_{k}+\left(z_{k+1}-z_{k}\right)\left(\frac{d y}{d z}\right)_{k} & \left(\frac{d y}{d z}\right)_{k+1}=\left(\frac{d y}{d z}\right)_{k}
\end{array}
$$

The $\frac{q}{p}$ parameter is predicted using the expected energy loss of a charged particle through the material between the states, which is calculated with the Bethe-Bloch formula. The additional error on $\frac{q}{p}$ is added to the predicted state. 


\section{Multiple Scattering}

Multiple Coulomb scattering will cause a particle to deviate from a straight line path. To account for this effect, we add noise to the covariance matrix of the predicted state. The noise matrix is [41]:

$$
\sigma^{2}\left(\theta_{\text {proj }}\right)\left(1+\left(\frac{d x}{d z}\right)^{2}+\left(\frac{d y}{d z}\right)^{2}\right)\left(\begin{array}{cccc}
(\delta z)^{2}\left(1+\left(\frac{d x}{d z}\right)^{2}\right) & (\delta z)^{2}\left(\frac{d x}{d z}\right)\left(\frac{d y}{z z}\right) & D(\delta z)\left(1+\left(\frac{d x}{2}\right)^{2}\right) & D(\delta z)\left(\frac{d x}{d z}\right)\left(\frac{d y}{d z}\right) \\
(\delta z)^{2}\left(\frac{d x}{d z}\right)\left(\frac{d y}{d z}\right) & (\delta z)^{2}\left(1+\left(\frac{d y}{d z}\right)^{2}\right) & D(\delta z)\left(\frac{d x}{d z}\right)\left(\frac{d y}{d z}\right) & D(\delta z)\left(1+\left(\frac{d y}{d z}\right)^{2}\right) \\
D(\delta z)\left(1+\left(\frac{d x}{d z}\right)^{2}\right) & D(\delta z))\left(\frac{d x}{d z}\right)\left(\frac{d y}{d z}\right) & \left(1+\left(\frac{d x}{d z}\right)^{2}\right) & \left(\frac{d x}{d z}\right)\left(\frac{d y}{d z}\right) \\
D(\delta z)\left(\frac{d x}{d z}\right)\left(\frac{d y}{d z}\right) & D(\delta z)\left(1+\left(\frac{d y}{d z}\right)^{2}\right) & \left(\frac{d x}{d z}\right)\left(\frac{d y}{d z}\right) & \left(1+\left(\frac{d y}{d z}\right)^{2}\right)
\end{array}\right)
$$

where all track parameters come from the previous state $\left(\vec{q}_{k}\right)$ and $D$ is - (+) for forward (backward) going tracks. $\sigma^{2}\left(\theta_{\text {proj }}\right)$ is the variance on the angular distribution of multiple scattering. Our approximation for $\sigma^{2}\left(\theta_{\text {proj }}\right)$ is the "much better approximation" from Lynch and Dahl [84]

$$
\sigma^{2}\left(\theta_{\text {proj }}\right)=\frac{\chi_{c}^{2}}{1+F^{2}}\left[\frac{1+\nu}{\nu} \ln (1+\nu)-1\right]
$$

where

$$
\begin{array}{r}
\nu=0.5 \frac{\Omega}{1-F} \\
\Omega=\frac{\chi_{c, e f f}^{2}}{\chi_{\alpha, e f f}^{2}}
\end{array}
$$

$\mathrm{F}$ is taken to be .98 (see section 3 of Lynch and Dahl). $\Omega$ is the mean number of scatters. $\chi_{c, e f f}^{2}$ and $\chi_{\alpha}^{2}$ account for a contributions from different nuclei and are effective versions of the Molière scattering angles $\chi_{c}^{2}$ and $\chi_{\alpha}^{2}$. The Molière scattering angles for a single nucleus are

$$
\begin{array}{r}
\chi_{c}^{2}=0.157[Z(Z+1) X / A][z /(p \beta)]^{2} \\
\chi_{\alpha}^{2}=2.007 \times 10^{-5} Z^{2 / 3}\left[1+3.34(Z z \alpha / \beta)^{2}\right] / p^{2}
\end{array}
$$

where $\mathrm{p}$ is the particle's momentum in $\mathrm{MeV} / c, \mathrm{z}$ is the charge of the particle, $\beta$ is the velocity of the particle, $\mathrm{X}$ is the path length in $\mathrm{g} / \mathrm{cm}^{2}, \mathrm{Z}$ and $\mathrm{A}$ are the atomic number and weight of

\footnotetext{
${ }^{1}$ The left side of this equation is $\sigma^{2}\left(\theta_{\text {proj }}\right)$. This corrects the acknowledged error in Equation 7 of Ref. [84]
} 
the scattering nucleus, and $\alpha=1 / 137$. For a particle passing through $N$ different nuclei, we construct the effective versions of these quantities:

$$
\begin{array}{r}
\chi_{c, e f f}^{2}=\sum_{i=1}^{N} c h i_{c, i}^{2} \\
\ln \left(\chi_{\alpha, e f f}\right)=\frac{\sum_{i=1}^{N} \frac{X Z(Z+1)}{A} \ln \left(\chi_{\alpha, i}\right)}{\sum_{i=1}^{N} \frac{X Z(Z+1)}{A}}
\end{array}
$$

\section{C.1.3 Filter}

The filter step compares state vector $i$ 's prediction for the next state at $i+1$ to the actual position of the cluster at $i+1$. The residual between the prediction and the measurement is used to calculate the Kalman gain and update the prediction for state $i+1$.

\section{C.1.4 Smooth}

The smoothing step uses the filtered prediction for state $i+1$ and an inverted covariance matrix to predict the previous state, at $i$. The filter process is repeated, this time to update state $i$.

\section{C.2 Vertex Fitting}

\section{C.2.1 Initial Guess}

Vertex fitting begins by making a quick guess for the vertex position based on points of closest approach (POCA) using a procedure described in Ref. [85]. Calculate the POCA for all permutations of pairs of tracks in the vertex. Assigning to the POCA between tracks $\mathrm{i}$ and $\mathrm{j}$ a weight of

$$
W_{i j}=\left(d_{\min }+D O C A\right)^{n}
$$


where DOCA is the distance of closest approach between tracks $\mathrm{i}$ and $\mathrm{j}$ in $\mathrm{mm}, d_{\min }=$ $0.01 \mathrm{~mm}$, and $n=-0.5$. The position of the vertex is taken to be the weighted average of POCAs.

\section{C.2.2 Adaptive Vertex Kalman Filter}

The adaptive Kalman filter applied to fitting a vertex is described in [85] and [86]. In each iteration of the filter, each track is considered as independent "measurement" used to update the vertex position. We use the position of the vertex and the track's slopes to predict where the track's nearest state should be and compare it to where the nearest track state really is. The residual of this comparision defines a Kalman gain, which is then used to upate the vertex position.

\section{Kalman Filter}

A linear track model is used to predict where the track's nearest state should be given the current guess of the vertex position and track momentum. Let " $h$ " be the predicted track parameters of a track coming out of a vertex at true position " $\mathrm{x}$ " with true momentum " $\mathrm{q}$ ". The equations that predict the track parameters are then

$$
\begin{array}{r}
\vec{h}(\vec{x}, \vec{q})=\overrightarrow{c_{0}}+A * \vec{x}+B * \vec{q} \\
A=\left.\frac{d \vec{h}}{d \vec{x}}\right|_{\vec{x}_{0}, \vec{q}_{0}} \\
B=\left.\frac{d \vec{h}}{d \vec{q}}\right|_{\vec{x}_{0}, \vec{q}_{0}} \\
\vec{c}_{0}=\vec{h}\left(\vec{x}_{0}, \vec{q}_{0}\right)-A * \vec{x}_{0}-B * \vec{q}_{0}
\end{array}
$$

where $\overrightarrow{x_{0}}$ and $\overrightarrow{q_{0}}$ are the position and slope of the state of the track closest to the vertex. The matrix A translates the vertex position into track parameter space, and B translates the track 
momentum into track parameter space. For a linear track model these equations become

$$
\begin{gathered}
A=\left(\begin{array}{cccc}
1 & 0 & -\left.\frac{d x}{d z}\right|_{\vec{x}_{0}, \vec{q}_{0}} \\
0 & 1 & -\left.\frac{d y}{d z}\right|_{\vec{x}_{0}, \vec{q}_{0}} \\
0 & 0 & 0 & \\
0 & 0 & 0 & \\
0 & 0 & 0 &
\end{array}\right) \\
B=\left(\begin{array}{ccc}
0 & 0 & 0 \\
0 & 0 & 0 \\
1 & 0 & 0 \\
0 & 1 & 0 \\
0 & 0 & 1
\end{array}\right) \\
\vec{c}_{0}=\left(\begin{array}{c}
\left.\frac{d x}{d z}\right|_{\vec{x}_{0}, \vec{q}_{0}} * z_{0} \\
\left.\frac{d y}{d z}\right|_{\vec{x}_{0}, \vec{q}_{0}} * z_{0} \\
0 \\
0 \\
0 \\
0 \\
0
\end{array}\right.
\end{gathered}
$$

The Kalman gain of the track is given by the product of the weight and inverse of the covariances of the estimated and measured track state:

$$
G=\text { weight } *\left(V_{\text {est. }}^{-1}+V_{\text {meas. }}^{-1}\right)
$$

This represents how much we trust the "measurement" of the nearest state on the track. The Kalman gain is used to update the covariance matrix, position of the vertex and momentum of tracks at the vertex according the the following formulae, respectively:

$$
\begin{array}{r}
C=\left(C_{\text {prev }}^{-1}+A^{T} * G_{b} * A\right)^{-1} \\
\vec{x}=C\left(C_{\text {prev }}^{-1} * \vec{x}_{\text {prev }}+A^{T} * G_{b} *\left(\vec{m}-\vec{c}_{0}\right)\right) \\
\vec{q}=W * B^{T} * G *\left(\vec{m}-\vec{c}_{0}-A * \vec{x}\right)
\end{array}
$$


where $\vec{m}$ is the fit parameters of the measured track state, and

$$
\begin{array}{r}
W=\left(B^{T} * G * B\right)^{-1} \\
G_{b}=G-G * B * W * B^{T} * G .
\end{array}
$$

The residual and $\chi^{2}$ added to the fit are

$$
\begin{array}{r}
\vec{r}=\left(\vec{m}-\vec{c}_{0}-A * \vec{x}-B * \vec{q}\right) \\
\chi_{k f}^{2}=\vec{r}^{T} * G * \vec{r}+\left(\vec{x}-\vec{x}_{\text {prev }}\right)^{T} * C_{\text {prev }}^{-1} *\left(\vec{x}-\vec{x}_{\text {prev }}\right)
\end{array}
$$

Track weights are then updated as

$$
\text { weight }_{i}=\frac{1}{1+e^{\frac{\chi_{i}^{2}-\chi_{c r i t}^{2}}{2.0 * T}}}
$$

where $\chi_{\text {crit }}^{2}$ is $10.0, \mathrm{~T}$ is the annealing temperature, and

$$
\chi_{i}^{2}=\operatorname{Similarity}(\vec{r}, G)+\operatorname{Similarity}\left(\vec{x}-\vec{x}_{\text {prev }}, C_{\text {prev }}^{-1}\right)
$$

One iteration of the filter represents the use of all tracks to update the vertex position and covariance matrix. The iterations are stopped due to convergence if the vertex position moves less than $0.1 \mathrm{~mm}$ or the $\chi^{2} / d o f$ improves by less than 0.05 . If after 10 iterations the fit has not converged, then we consider the fit failed.

\section{C.2.3 Annealing}

The annealing process is used to increase the effects of track weighting upon successive iterations of the filter. As our fit for the vertex position gets better with each fit, we are more confident of whether a track is or is not compatible with a track coming from the vertex, so the downweighting of non-compatible tracks becomes more severe with each iteration. The annealing temperature is set to an initial value of 25 , and is updated after each iteration of the Kalman filter accoring the the formula [42]

$$
T=1.0+0.35 *\left(T_{\text {prev }}-1.0\right)
$$




\title{
Appendix D
}

\section{The MINERvA Collaboration}

\author{
G. Tzanakos \\ University of Athens \\ J. Cravens, M. Jerkins, S. Kopp, J. Ratchford \\ University of Texas at Austin \\ D.A.M. Caicedo, C.M. Castromonte, H. da Motta, G.A. Fiorentini, K. Hurtado, J.L. Palomino \\ Centro Brasileiro de Pesquisas Físicas \\ D.W. Schmitz \\ University of Chicago \\ J. Grange, J. Mousseau, B. Osmanov, H. Ray \\ University of Florida \\ D. Boehnlein, R. DeMaat, N. Grossman, D.A. Harris, J.G. Morfín, J. Osta, E. A. Paschos, \\ L. Rakotondravohitra, P. Rubinov, F.D. Snider, R. Stefanski \\ Fermilab
}


A. Blondel, A. Bravar, Y. Karadzhov, A. Korzenev, C. Martin Mari University of Geneva

J. Felix, A. Higuera, Z. Urrutia, E. Valencia, G. Zavala

Universidad de Guanajuato

M.E. Christy, M. Datta, C. Keppel, W. Tan, T. Walton, L. Y. Zhu

Hampton University

A. Butkevich, S.A. Kulagin

Inst. Nucl. Reas. Moscow

G. Niculescu, I. Niculescu

James Madison University

E. Maher

Mass. Col. Lib. Arts

L. Fields, B. Gobbi, J.A. Hobbs, C.E. Patrick, L. Patrick, H. Schellman

Northwestern University

N. Tagg

Otterbein University

S. Boyd, S.A. Dytman, B. Eberly, Z. Isvan, C.L. McGivern, D. Naples, V. Paolone, L. Ren University of Pittsburgh 


\title{
G.A. Díaz , A.M. Gago, J.P. Velásquez \\ Pontificia Universidad Catolica del Peru
}

\author{
R. Napora \\ Purdue University Calumet
}

S. Avvakumov, A. Bodek, R. Bradford, H. Budd, J. Chvojka, M. Day, H. Lee, L. Loiacono, S. Manly, C.M. Marshall, K.S. McFarland, A.M. McGowan, A. Mislivec, J. Park, G.N. Perdue,

P.A. Rodrigues, J. Wolcott

University of Rochester

G. J. Kumbartzki, T. Le, R.D. Ransome, E.C. Schulte, B.G. Tice Rutgers University

O. Altinok, H. Gallagher, T. Kafka, W.A. Mann, W. P. Oliver Tufts University

\author{
C. Simon, B.P.Ziemer \\ University of California at Irvine
}

R. Gran, M. Lanari

University of Minnesota at Duluth

M. Alania, C.J. Solano Salinas

Universidad Nacional de Ingeniería 
W.K. Brooks, G. Maggi, J. Miller, C. Peña, I.K. Potashnikova, F. Prokoshin Universidad Técnica Federico Santa María

L. Aliaga, J. Devan, M. Kordosky, J.K. Nelson, J. Walding, D. Zhang College of William and Mary 


\section{Bibliography}

[1] Q.R. Ahmad et al. Direct evidence for neutrino flavor transformation from neutral-current interactions in the sudbury neutrino observatory. Phys. Rev. Lett., 89(1):011301, (2002). 4

[2] R. Davis Jr., D.S. Harmer, and K.C. Hoffman. Search for neutrinos from the sun. Phys. Rev. Lett., 20(21):1205, (1968). 4

[3] D.J. Griffiths. Introduction to Elementary Particles, chapter 11. WILEY-VCH Verlag GmbH \& Co., (2008). 4

[4] J.Jo. Aubert et al. (EMC). The ratio of the nucleon structure functions $f_{2}^{N}$ for iron and deuterium. Physics Letters B, 123(3):275-278, (1983). 5

[5] C.H. Llewellyn Smith. Neutrino reactions at accelerator energies. Phys. Rept., 3:261-379, (1972). 7, 58

[6] K.S. Kuzmin, V.V. Lyubushkin, and V.A. Naumov. arxiv:0712.4384v3[hep-ph]. (2008). 7

[7] A. Bodek, S. Avvakumov, R. Bradford, and H. Budd. arxiv:0709.3538v1[hep-ex]. (2007). 7

[8] M. Gell-Mann and M. Levy. The axial vector current in beta decay. Il Nuovo Cimento, 16(4):705-726, (1960). 8

[9] D. Rein and L.M. Sehgal. Neutrino-excitation of baryon resonances and single pion production. Annals of Physics, 133(1):79-153, (1981). 8, 60

[10] J.A. Formaggio and G.P. Zeller. From eV to EeV: Neutrino Cross Sections Across Energy Scales. Rev. Mod. Phys. 84, 1307, (2012). 9

[11] M. Arneodo. Nuclear effects in structure functions. Phys. Rept., 240:301, 1994.

[12] P.R. Norton. The EMC effect. Rept. Prog. Phys., 66:1253-1297, 2003.

[13] B.Z. Kopeliovich, J.G. Morfín, and I. Schmidt. Nuclear shadowing in electro-weak interactions. Prog. Part. Nucl. Phys., (2012). 12, 126

[14] M.R. Adams et al. Shadowing in inelastic scattering of muons on carbon, calcium and lead at low $x_{B j}$. Z. Phys. C, 67(3):403-410, 1995.

[15] P.P. Allport et al. Observation of shadowing of neutrino- and antineutrino-nucleus interactions and comparison with PCAC predictions. Phys. Lett. B, 232(3):417 - 424, 1989. 
[16] D.F. Geesaman, K. Saito, and A.W. Thomas. The nuclear EMC effect. Annu. Rev. Nucl. Part. Sci., 45(1):337-390, (1995). 13

[17] Z. Ahmed et al. New precision limit on the strange vector form factors of the proton. Phys. Rev. Lett., 108(10):102001, (2012). 13

[18] B. Povh, K. Rith, C. Scholz, and F. Zetsche. Particles and Nuclei, chapter 17. SpringerVerlag Berlin Heidelberg, (2008). 13

[19] R.A. Smith and E.J. Moniz. Neutrino reactions on nuclear targets. Nuclear Physics B, 43:605-622, (1972). 13,57

[20] A. Bodek and J.L. Ritchie. Fermi-motion effects in deep-inelastic lepton scattering from nuclear targets. Phys. Rev. D, 23(5):1070, (1981). 14

[21] A. Bodek, H.S. Budd, and M.E. Christy. Neutrino quasielastic scattering on nuclear targets. Eur. Phys. J. C, 71(9):1-18, (2011). 14, 127

[22] R. Subedi, R. Shneor, P. Monaghan, et al. Probing cold dense nuclear matter. Science, 320(5882):1476-1478, (2008). 14

[23] S. Boyd, S. Kulagin, J.G. Morfín, and R.D. Ransome. Studying nuclear effects with neutrinos (MINERvA internal note 700). (2004). URL http://minerva. fnal. gov/notes/minnote_700_nuc.pdf. 15

[24] C. Andreopoulos et al. (GENIE collaboration). The GENIE Neutrino Monte Carlo Generator. Nucl. Inst. and Meth., Phys. Res. Sect. A, 614(1):87-104, (2010). 15, 57, 59, 105, 116

[25] D.A. Crane et al. Status report: Technical design of neutrino beams for the Main Injector (NuMI). FERMILAB-TM-1946, NUMI-B-92, FERMILAB-DESIGN-1995-03, (1995). 16

[26] L. Aliaga et al. (MINERvA collaboration). Design, calibration, and performance of the MINERvA detector . Nucl. Inst. and Meth., Phys. Res. Sect. A, 743(0):130 - 159, 2014. 16, 34, 35, 37, 40, 41, 42, 44, 102, 104, 107, 108, 111, 112, 115

[27] D.G. Michael et al. (MINOS collaboration). The Magnetized steel and scintillator calorimeters of the MINOS experiment. Nucl. Inst. and Meth., Phys. Res. Sect. A, 596:190-228, (2008). 16, 30, 100

[28] C. Anderson et al. (Argoneut collaboration). The ArgoNeuT Detector in the NuMI LowEnergy beam line at Fermilab. arXiv:1205.6747 [hep-ex], (2012). 16

[29] R.B. Patterson. The NOvA experiment: status and outlook. Nucl. Phys. B, Proc. Suppl., 235:151-157, (2013). 16

[30] R. Zwaska. Accelerator Systems and Instrumentation for the NuMI Neutrino Beam. Ph.D. thesis, FERMILAB-THESIS-2005-73, University of Texas at Austin, (2005). 17

[31] Fermi National Accelerator Laboratory. The Fermilab Main Injector Technical Design Handbook. FERMILAB-DESIGN-1994-01, (1994). 17 
[32] R. Zwaska et al. Beam-based alignment of the numi target station components at fnal. Nucl. Inst. and Meth., Phys. Res. Sect. A, 568(2):548 - 560, (2006). 18, 19, 20

[33] K. Budal. Measurement of Charge Emission from Targets as a Means of Burst Intensity and Beam Intensity Monitoring. IEEE Trans. Nucl. Sci., 14:1132, (1967). 18

[34] M. Kordosky. NuMI (MINERvA) flux prediction, (2011). URL http://indico. cern.ch/contributionDisplay.py?contribId=116\&sessionId= 2\&confId=114816. NuFact '11, XIIIth International Workshop on Neutrino Factories, Super beams and Beta beams. 18, 19,99

[35] Fermi National Accelerator Laboratory. The Main Injector Technical Design Handbook, Chapter 2. (2002). URL http://www-numi.fnal.gov/numwork/tdh/tdh_ index.html, 18

[36] J. Beringer et al. (Particle Data Group). Review of particle physics. Phys. Rev. D, 86:010001, (2012). 19

[37] G.N. Perdue et al. (MINERvA collaboration). The MINERvA data acquisition system and infrastructure. Nucl. Inst. and Met., Phys. Res. Sect. A, 694(0):179 - 192, (2012). 30

[38] P. Adamson et al. (MINOS collaboration). Neutrino and antineutrino inclusive chargedcurrent cross section measurements with the minos near detector. Phys. Rev. D, 81(7):072002, (2010). 31

[39] J. Chvojka. Anti-neutrino charged current quasi-elastic scattering in MINERvA. Ph.D. thesis, FERMILAB-THESIS-2012-22, Rochester University, (2013). 31

[40] R. Frühwirth. Application of kalman filtering to track and vertex fitting. Nucl. Inst. and Meth., Phys. Res. Sect. A, 262(2-3):444 - 450, (1987). 48, 69, 236

[41] E.J. Wolin and L.L. Ho. Covariance matrices for track fitting with the Kalman filter. Nucl. Inst. and Meth., Phys. Res. Sect. A, 329:493-500, (1993). 48, 238

[42] W. Waltenberger. Adaptive vertex reconstruction. Technical Report CMS-NOTE-2008033, CERN, Geneva, (2008). 49, 242

[43] D.E. Groom, N.V. Mokhov, and S.I. Striganov. Muon stopping power and range tables 10 MeV-100 TeV. Atomic Data and Nuclear Data Tables, 78(2):183-356, (2001). 52

[44] NA49 collaboration. NA49 Homepage. (1997). URL http://na49info.web. cern.ch/na49info/, 55, 56, 98

[45] D.S. Barton et al. Experimental study of the a dependence of inclusive hadron fragmentation. Phys. Rev. D, 27(11):2580, (1983). 55, 56

[46] A. Ferrari, P.R. Sala, A. Fasso, and J. Ranft. Fluka. CERN-library in: http://fluka. web. cern. ch/fluka, (2005). 55, 99,100

[47] R.P. Feynman, R.D. Field, and G.C. Fox. Quantum-chromodynamic approach for the large-transverse-momentum production of particles and jets. Phys. Rev. D, 18(9):3320, (1978). 56 
[48] R. Bradford, A. Bodek, H. Budd, and J. Arrington. A new parameterization of the nucleon elastic form factors. Nucl. Phys. Proc. Suppl., 159:127-132, (2006). 58

[49] S.L. Adler. Tests of the conserved vector current and partially conserved axial-vector current hypotheses in high-energy neutrino reactions. Phys. Rev., 135(4B):B963, (1964). 60

[50] A. Bodek, I. Park, and U.K. Yang. Improved low $Q^{2}$ model for neutrino and electron nucleon cross sections in few-GeV region. arxiv:0411202[hep-ph], (2004). 61

[51] A. Bodek and U.K. Yang. Modeling neutrino and electron scattering inelastic cross sections. arXiv:0308007[hep-ex], (2003). 61

[52] A. Bodek and U.K. Yang. Modeling deep inelastic cross sections in the few GeV region. Nuclear Physics B-Proceedings Supplements, 112(1):70-76, (2002). 61

[53] T. Yang, C. Andreopoulos, H. Gallagher, K. Hoffmann, and P. Kehayias. A hadronization model for few-GeV neutrino interactions. Eur. Phys. J. C, 63(1):1-10, (2009). 62

[54] T. Yang, C. Andreopoulos, H. Gallagher, K. Hoffmann, and P. Kehayias. A Hadronization Model for Few-GeV Neutrino Interactions. Eur.Phys.J., C63:1-10, (2009). 62

[55] T. Sjöstrand et al. . High-energy-physics event generation with pythia 6.1. Computer Physics Communications, 135(2):238-259, (2001). 62

[56] T. Sjöstrand and M. Bengtsson. The Lund Monte Carlo for jet fragmentation and $e^{+} e^{-}$ physics-jetset version 6.3-an update. Computer Physics Communications, 43(3):367$379,(1987) .63$

[57] S. Agostinelli et al. (Geant4 collaboration). G4-a simulation toolkit. Nucl. Inst. and Meth., Phys. Res. Sect. A, 506(3):250 - 303, (2003). 64

[58] J.B. Birks. Scintillations from organic crystals: specific fluorescence and relative response to different radiations. Proceedings of the Physical Society. Section A, 64(10):874, (1951). 65

[59] R. Brun, A.C. McPherson, P. Zanarini, M. Maire, and F. Bruyant. GEANT 3 : user's guide Geant 3.10, Geant 3.11. CERN-DD-EE-84-01, (1987). 65

[60] J.S. Marshall. A study of muon neutrino disappearance with the MINOS detectors and the NuMI neutrino beam. Ph.D. thesis, FERMILAB-THESIS-2008-20, University of Cambridge, (2008). 68

[61] B.G. Tice. Vertex quality in the nuclear targets inclusive analysis. MINERvA Internal Note TN35, (2013). 70

[62] T. Adye. Unfolding algorithms and tests using roounfold. arXiv:1105.1160, (2011). 85, 114

[63] D.W. Schmitz, G.A. Fiorentini, T. Le, L. Rakotondravohitra, and B.G. Tice. Reconstruction efficiency corrections for the titan production pass. MINERvA Internal Note TN16, (2013). 86 
[64] B.G. Tice. Post-framework analysis histograms for MINERvA. MINERvA Internal Note TN22, (2013). 95

[65] Y. Fisyak et al. (MIPP collaboration). P-907: Proposal to measure particle production in the meson area using main injector primary and secondary beams. Proposal to the FNAL PAC, (2000). 98

[66] N. Abgrall et al. (NA61/SHINE collaboration). Measurements of Cross Sections and Charged Pion Spectra in Proton-Carbon Interactions at $31 \mathrm{GeV} / \mathrm{c}$. Phys. Rev. C, 84:034604, (2011). 98

[67] J. Wolcott. Uncertainties due to Feynman scaling of NA49 data to NuMI energy. MINERvA Internal Note TN15, (2013). 98

[68] Geant4: Reference physics lists. (2013). URL http://geant4.cern.ch/ support/proc_mod_catalog/physics_lists/referencePL.shtml. 99

[69] Ž. Pavlović. Observation of disappearance of muon neutrinos in the NuMI beam. Ph.D. thesis, FERMILAB-THESIS-2008-59, University of Texas at Austin, (2008). 99, 100, 101

[70] S.A. Dytman, H. Gallagher, and M. Kordosky. Hadronic shower energy scale uncertainty in the minos experiment. arXiv:0806.2119[hep-ex], (2008). 106

[71] J. Boehm, H. Gallagher, and T. Yang. Hadronization uncertainties for the $\nu_{e}$ analysis (MINOS docdb 5392). docdb-7448v1, (2008). 107

[72] R. Gran. Proton range, birks constant. docdb-8945v1, (2013). 108

[73] Fermilab Test Beam Facility. Fermilab test beam facility. (2013). URL http: //www-ppd.fnal.gov/ftbf/, 108

[74] J.P. Velásquez, S.A. Dytman, and A.M. Gago. Geant4 hadronic uncertainties. docdb8249v1, (2012). 109, 110

[75] W.P. Abfalterer et al. Measurement of neutron total cross sections up to $560 \mathrm{MeV}$. Phys. Rev. C, 63(4):044608, (2001). 109

[76] M. Ibaraki et al. Measurement of Neutron Non-elastic Cross Sections of C, Si, Fe, Zr, and $\mathrm{Pb}$ in 40-80 MeV Region. Journal of Nucl. Sci. and Tech., 2:405-408, (2002). 109

[77] W. Schimmerling et al. Neutron-Nucleus Total and Inelastic Cross Sections: 900 to 2600 $\mathrm{MeV} /$ c. Phys. Rev. C, 7(1):248, (1973). 109

[78] R.G.P. Voss and R. Wilson. The analysis of high-energy neutron cross-sections. Proceedings of the Royal Society of London. Series A. Mathematical and Physical Sciences, 236(1204):52-67, (1956). 109

[79] C.M. Marshall, A. Mislivec, and R. Gran. Uncertainties in the detector energy scale for the testbeam. docdb-7525v4, (2012). 111

[80] J. Wolcott. Cross-talk in MINERvA. MINERvA Internal Note TN3, (2012). 113 
[81] J. E. Amaro, M. B. Barbaro, J. A. Caballero, T. W. Donnelly, and C. F. Williamson. Meson-exchange currents and quasielastic neutrino cross sections in the superscaling approximation model. Phys. Lett. B, 696(12):151 - 155, 2011. 127

[82] J. Nieves, I. Ruiz Simo, and M. J. Vicente Vacas. Inclusive charged-current neutrinonucleus reactions. Phys. Rev. C, 83:045501, Apr 2011. 127

[83] R. Gran, J. Nieves, F. Sanchez, and M. J. Vicente Vacas. Neutrino-nucleus quasi-elastic and 2p2h interactions up to $10 \mathrm{GeV}$. Phys. Rev. D, 88:113007, Dec 2013. 127

[84] G.R. Lynch and O.I. Dahl. Approximations to multiple coulomb scattering. Nucl. Inst. and Meth., Phys. Res. Sect. B, 58(1):6 - 10, (1991). 238

[85] R. Frühwirth, W. Waltenberger, and P. Vanlaer. Adaptive vertex fitting. Technical Report CMS-NOTE-2007-008, CERN, Geneva, (2007). 239, 240

[86] R. Luchsinger and C. Grab. Vertex reconstruction by means of the method of kalman filtering. Computer Physics Communications, 76(3):263 - 280, (1993). 240 